\title{
HYBRIDIZING NICKEL PLATFORM USING ULTRA-SHORT PULSED LASER: A NEW PARADIGM TOWARDS BIOMEDICAL SENSING AND THERANOSTICS
}

\author{
by \\ Sivaprasad Chinnakkannu Vijayakumar \\ Master of Applied Science in Mechanical and Industrial Engineering, \\ Ryerson University, Canada, 2014 \\ Bachelor of Engineering in Mechanical Engineering, \\ Anna University, India, 2011 \\ A dissertation presented to Ryerson University \\ in partial fulfillment of the \\ requirements for the degree of \\ Doctor of Philosophy \\ in the program of \\ Mechanical and Industrial Engineering \\ Toronto, Ontario, Canada, 2018 \\ (C) Sivaprasad Chinnakkannu Vijayakumar, 2018
}




\section{AUTHOR'S DECLARATION}

I hereby declare that I am the sole author of this dissertation. This is a true copy of the dissertation, including any required final revision, as accepted by my examiners.

I authorize Ryerson University to lend this dissertation to other institutions or individuals solely for the purpose of scholarly research.

I further authorize Ryerson University to reproduce this dissertation by photocopy or by other means, in total or in part, at the request of other institutions or individuals for the purpose of scholarly research.

I understand that my dissertation may be made electronically available to the public. 


\title{
Abstract \\ HYBRIDIZING NICKEL PLATFORM USING ULTRA-SHORT PULSED LASER: A NEW PARADIGM TOWARDS BIOMEDICAL SENSING AND THERANOSTICS
}

\author{
Sivaprasad Chinnakkannu Vijayakumar \\ Doctor of Philosophy, 2018 \\ Mechanical and Industrial Engineering, Ryerson University
}

\begin{abstract}
A self-assembled 3D nanonetwork of Nickel and Nickel Oxide is synthesized by ultrashort pulsed laser through multiphoton ionization. The synthesized nanonetwork with tunable physiochemical property was investigated for cancer therapeutic and biomolecular sensing applications. In this thesis, the developed 3D nickel nanomatrix effectively regulated HeLa cancer cell adhesion and proliferation mimicking Extracellular Matrix (ECM). This behaviour explicitly demonstrated that the initial incubation period was devoted to baiting fibroblast and HeLa cells to proliferate upon the nanomatrix and subsequently the same nanomatrix exhibited cell trapping behaviour upon HeLa cells after an increased incubation period thereby controlling proliferation. The results brought new insight as to how HeLa cells behaved differently when compared to NIH3T3 fibroblast cells opening pioneering application in drug-free cancer therapy. To delve deeper into nickel nanonetwork for cancer therapy the laser ionization was manipulated to induce two distinct quantum theranosomes. Presently, quantum materials are limited due to OD \& 1D materials lacking biocompatibility resulting in coated materials with labelled tags for fluorescence excitation. The theranosomes mimicked tumor microenvironment by selectively accelerating the proliferation of mammalian fibroblasts cells while inducing cancer therapy. Furthermore, the theranosomes opened up label-free bioimaging probe for differentiating (HeLa \& MDAMB-231) from mammalian fibroblast cells for cancer diagnostics. In-addition to label-free bioimaging, the development of an ultrasensitive biosensor for targeted biomolecule sensing was developed addressing the drawback faced with fluorescence
\end{abstract}


imaging using Surface Enhanced Raman Scattering (SERS). We developed a SERS active nano-biosensor to detect chemical dye Crystal Violet (CV) and biomolecule glutathione(GSH). The Raman detection of crystal violet $(\mathrm{CV})$ and glutathione $(\mathrm{GSH})$ molecules was noted with $1 \mathrm{pM}\left(1 \times 10^{-12} \mathrm{M}\right)$ concentrations at $(532$ \& $785 \mathrm{~nm}$ ) excitation wavelengths with an enhancement factor of $10^{9}$, not been observed even in plasmonic materials. This extends the limit of detection (LOD), confirming suitability for chemical and biomolecular sensing. Additionally, the quantum confinement effect will result in an ultrasensitive sensor diagnosing and differentiating cancer cells from fibroblast cells. Based on the results in this thesis, the multifunctional feasibility of nano and quantum scale nickel structures arranged in 3D assembly for its direct application in cancer therapeutics, encompassing cancer bioimaging and diagnostics. 


\section{ACKNOWLEDGEMENTS}

I would like to express my profound gratitude and appreciation for my supervisors Dr. Krishnan Venkatakrishnan and Dr. Bo Tan who were both instrumental in first encouraging me to venture into a field that is new evolving and providing me with a platform full of opportunities and assuring me of their unwavering support at all times. Their continued help, advice and boundless support has supported me in being a better researcher and has given me the necessary skills for future endeavours. It is now very appropriate to mention at this stage that Ryerson University has been a key factor for my continuing the Doctoral program after completion of my master's for the positive atmosphere I was placed in and for the grants awarded.

I would like to thank my committee members Dr. Ahmad Ghasempoor, Dr. Vincent Chan and Dr. Ziad Saghir for their valuable guidance in shaping the direction of my research project and refining my thesis.

I would like to express my deepest gratitude to my parents Devi and Vijayakumar for their patience, lending their sympathetic ear, providing wise counsel whenever I doubted myself and their constant encouragement with financial support when needed which helped me in successful completion of this dissertation. It is appropriate now to record that my elder brother Chandramouli Chinnakkannu Vijayakumar was a pillar of strength and I thank him profusely for sparing his time to engage me in technical debates, deliberating over problems encountered and confronting me with probing questions which helped me in honing my existing research skills.

I would also like to thank all my past and present lab mates for all of their advice, as well as the staff of the Mechanical and Industrial Engineering department for all the help they provided. 


\section{DEDICATION}

To my parents and my elder brother. 


\section{TABLE OF CONTENTS}

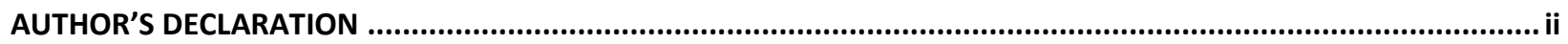

ABSTRACT

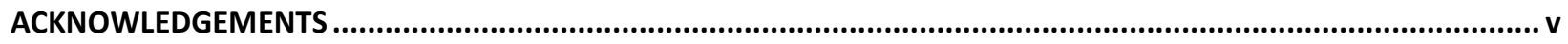

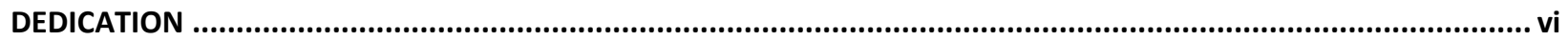

LIST OF TABLES

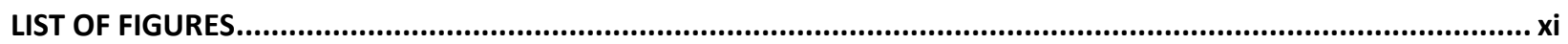

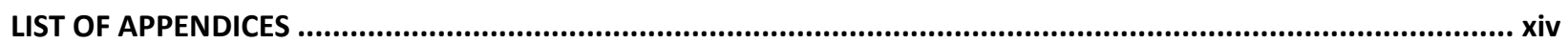

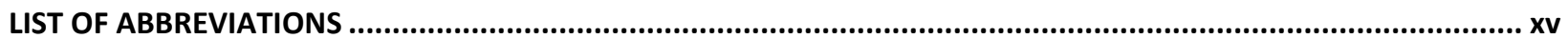

LIST OF NOMENCLATURE ....................................................................................................................................

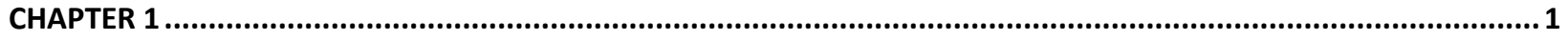

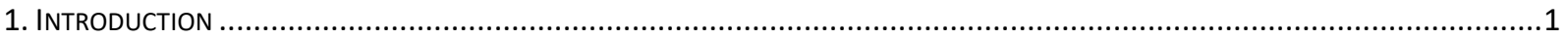

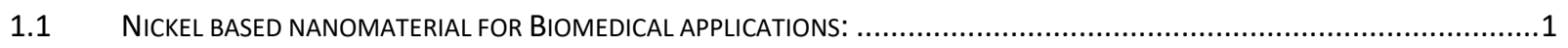

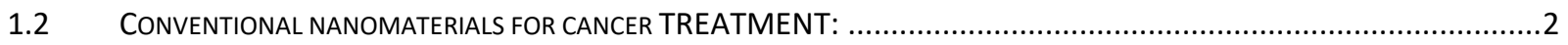

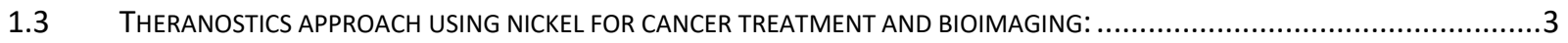

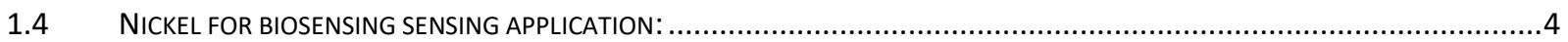

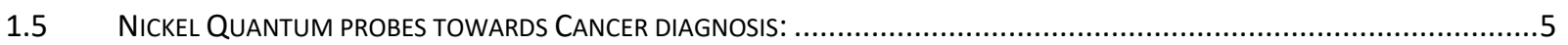

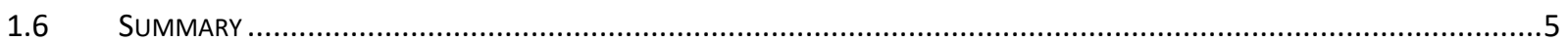

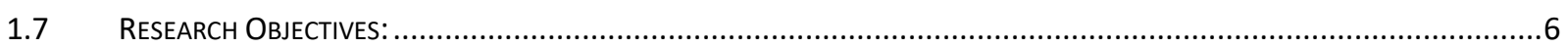

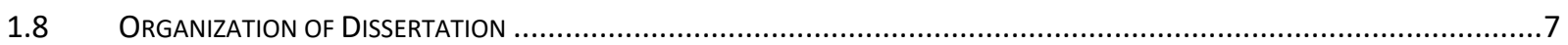

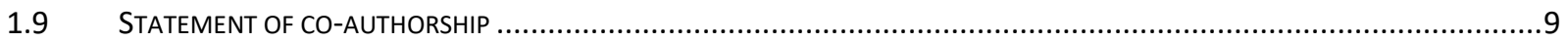

CHAPTER 2

SELECTIVE DRUG-FREE CANCER APOPTOSIS BY THREE-DIMENSIONAL SELF-TARGETING MAGNETIC NICKEL OXIDE

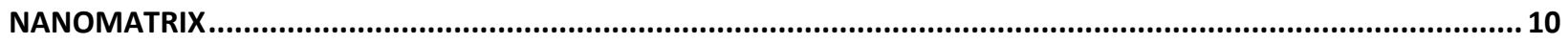

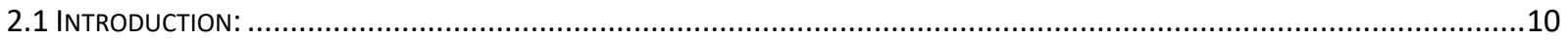

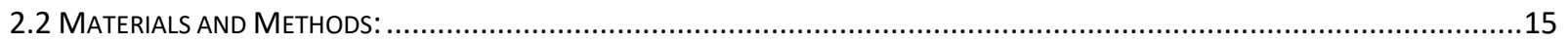

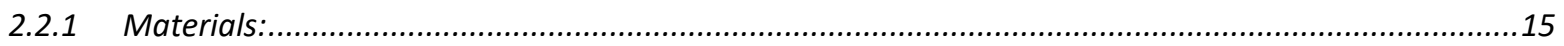

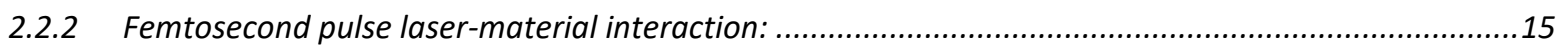

2.2.3 Nanomatrix morphological and physiochemical characterization: .....................................................16

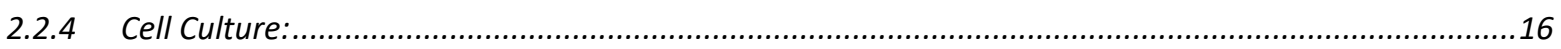

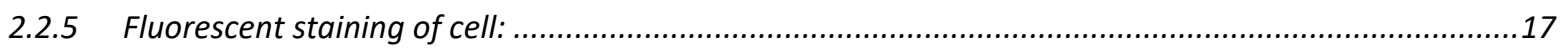

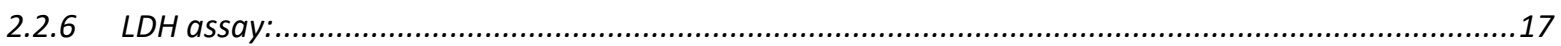

2.2.7 Statistics:

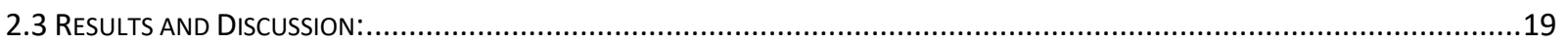

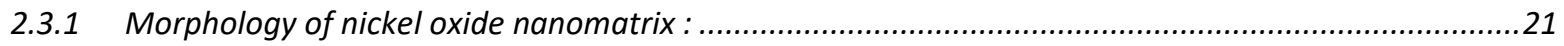

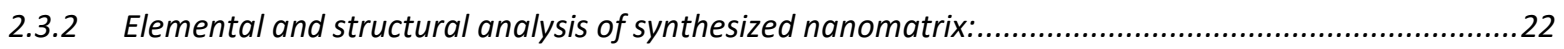

2.3.3 Effect of $L D H$ release of HeLa and NIH3T3 cell in self-assembled nanomatrix: ...................................23

2.3.4 HeLa and fibroblast cell response on synthesized nickel oxide nanomatrix platforms: ........................24

2.3.5 Role of nanomatrix morphology and chemistry in cell-nanomaterial interaction:..................................30

2.3.6 Effect of different shaped nanomatrix on cell morphological characteristics: ....................................33

2.3.7 Physiological study of interacting cells with synthesized nanomatrix: .............................................36 


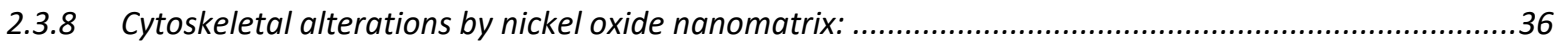

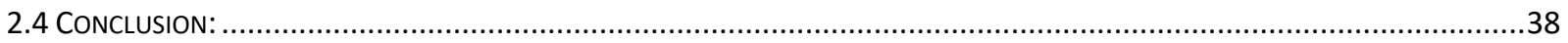

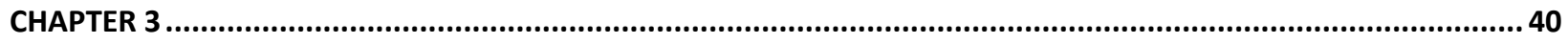

3D QUANTUM THERANOSOMES: A NEW DIRECTION FOR LABEL-FREE THERANOSTICS.................................40

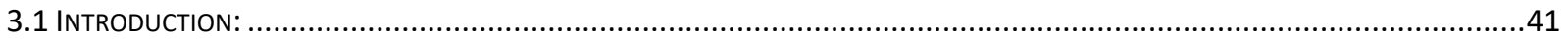

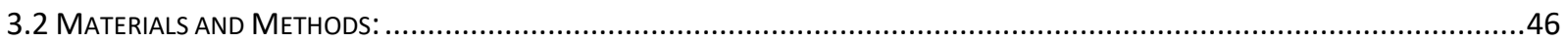

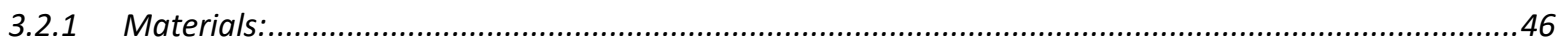

3.2.2 Laser ionization to synthesize theranosomes: .............................................................................46

3.2.3 Synthesis of theranosomes by multiphoton ionization mechanism of solid nickel substrate: ...............46

3.2.4 Theranosomes morphological and physiochemical characterization: ................................................48

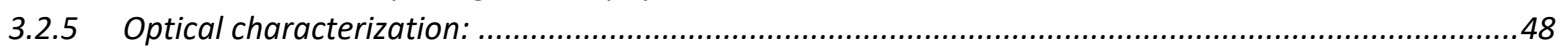

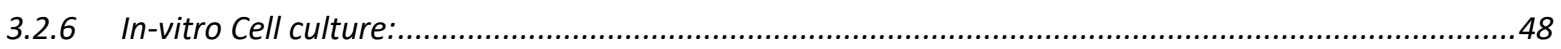

3.2.7 Label-free Fluorescence Bioimaging: ....................................................................................49

3.2.8 Differential fluorescence staining of cells: ...................................................................................50

3.2.9 Assessment of necrosis and apoptosis using Annexin V - PI staining:............................................50

3.2.10 Reactive oxygen species (ROS)determination using flow cytometry and fluorescence microscopy: ......51

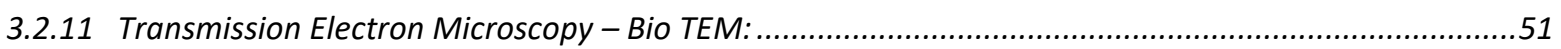

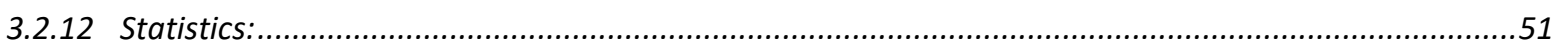

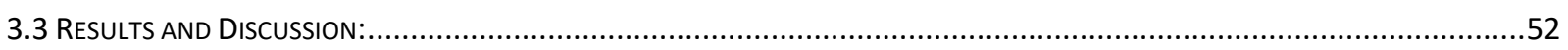

3.3.1 Morphological and material chemistry investigation of theranosomes: ............................................52

3.3.2 Optical(UV-Vis) and Magnetic (Resonance Properties using EPR) characterization:............................58

3.3.3 Quantum Theranosomes fluorescence enhancement intensity using Flow Cytometry and

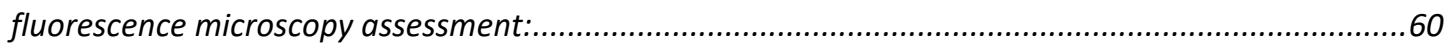

3.3.4 In-vitro fluorescence bioimaging property of theranosomes:......................................................63

3.3.5 In-vitro therapeutic efficacy of theranosomes:..........................................................................70

3.3.6 Intracellular Reactive Oxygen Species (ROS) assessment of Quantum Theranosomes: ........................75

3.3.7 Cell death induction by 3D quantum theranosomes and assessment of Necrosis and apoptosis: ........ 77

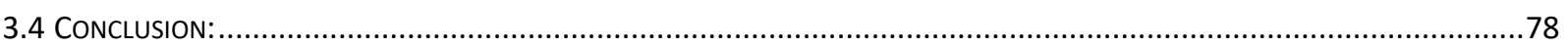

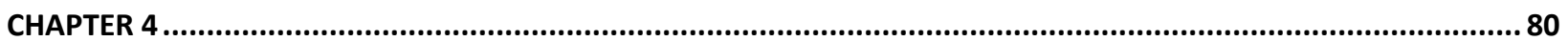

\section{SERS ACTIVE NANOBIOSENSOR FUNCTIONALIZED BY SELF-ASSEMBLED 3D NICKEL NANONETWORKS FOR}

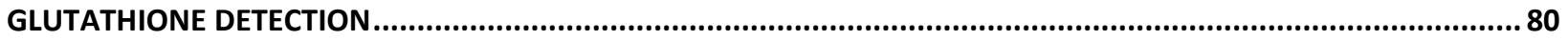

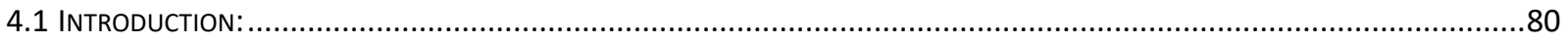

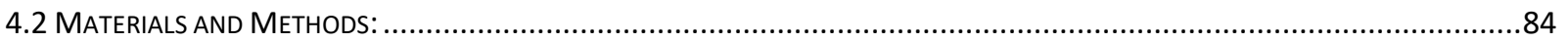

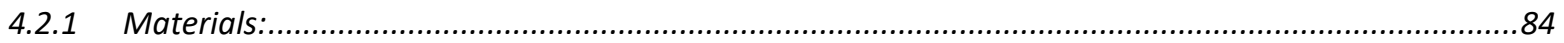

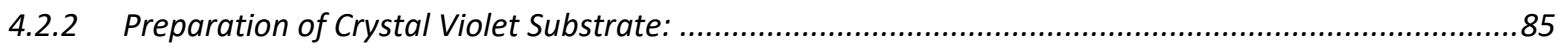

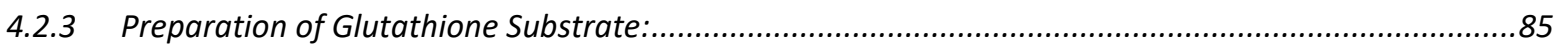

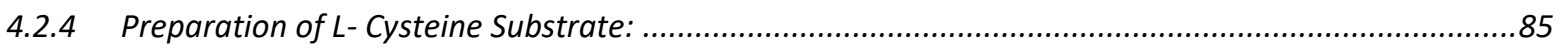

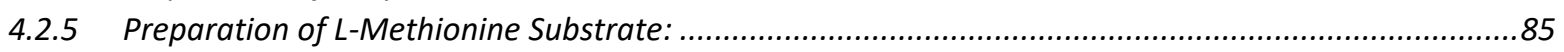

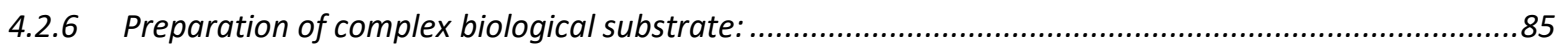

4.2.7 Preparation of GSH with complex biological substrate: .................................................................85

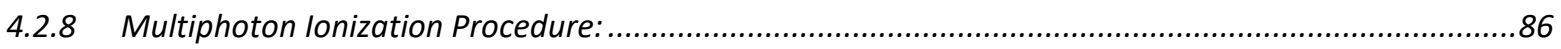

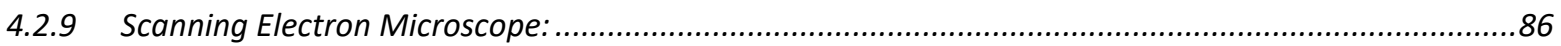

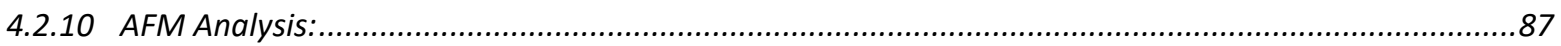

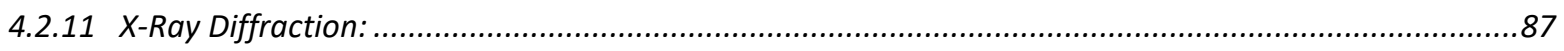

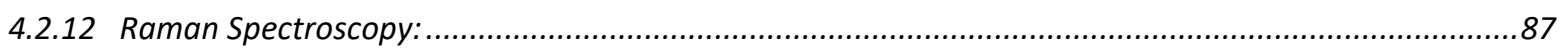

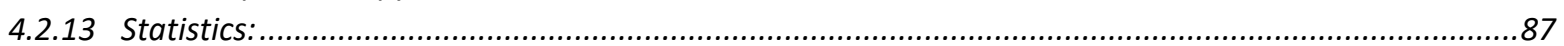




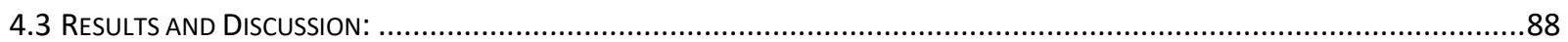

4.3.1 Synthesizing Interdenominational Self-assembled 3D Hybrid Nickel Nanonetwork: .............................88

4.3.2 Structure, morphology, and physical property analysis of the synthesized nanonetwork:...................89

4.3.3 Self-assembled nanonetwork material properties and SERS Enhancement: .....................................92

4.3.4 Raman scattering effect of CV on Nickel nanonetwork: .............................................................93

4.3.5 Realizing biomolecule detection on nickel biochip:..................................................................94

4.3.6 Establishing nano-biosensor sensitivity and limit of detection: .........................................................99

4.3.7 Accomplishing L-Cysteine and L-Methionine detection on nickel nano chips: ...................................103

4.3.8 Accomplishing GSH sensing in complex biological environment detection on nickel nano chips: ........105

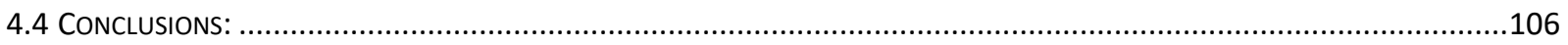

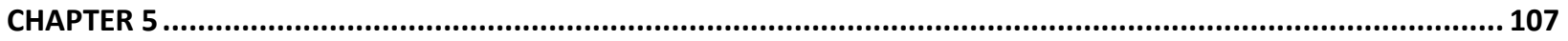

ATOMIC ANTENNAS ON PORTABLE QUANTUM STRUCTURES - A NON -TERS APPROACH ...............................107

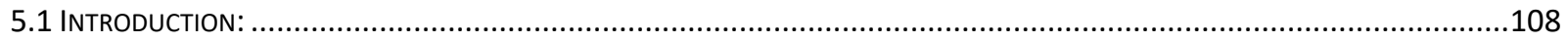

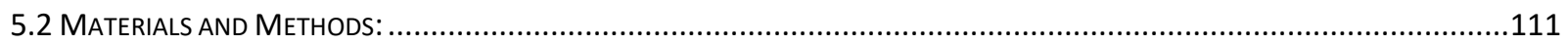

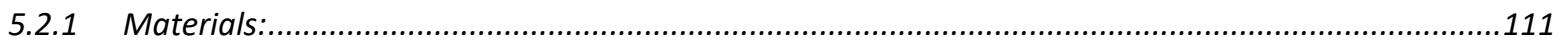

5.2.2 Synthesis of quantum probes:................................................................................................. 112

5.2.3 Physicochemical characterization of quantum probes: .................................................................112

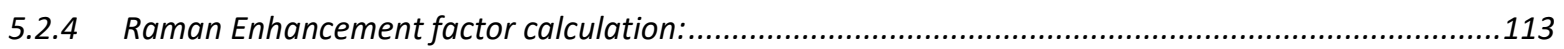

5.2.5 Optical characterization of quantum probes: ............................................................................115

5.2.6 Preparation of Raman probe molecules (Crystal violet (CV) and Rhodamine 6G (R6G)): ..................115

5.2.7 In-vitro cell culture for Transmission Electron Microscopy: ......................................................115

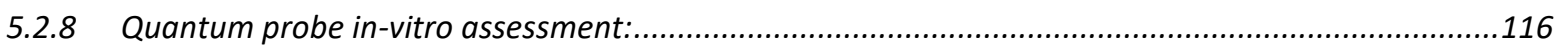

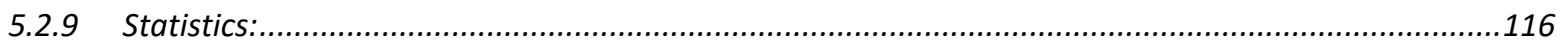

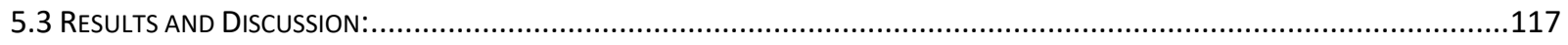

5.3.1 Synthesis of quantum probe by ultra-short ionization mechanism of solid nickel substrate:..............117

5.3.2 Raman scattering effect of Crystal Violet (CV) on quantum probes: ................................................122

5.3.3 Raman scattering effect of Rhodamine 6G (R6G) on quantum probes: ..........................................124

5.3.4 Raman scattering effect of Bovine Serum Albumin (BSA) biomolecules and label-free cell adhesion and quantum probe uptake in cells: .......................................................................................125

5.3.5 In-vitro raman response to portable quantum probes for cancer detection using protein signature: 128

5.3.6 Cancer cell differentiation using Raman response from portable quantum probes: ..........................130

5.3.7 Cancer monitoring/therapeutics efficiency:...........................................................................132

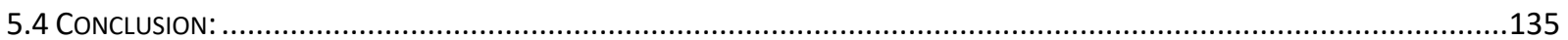

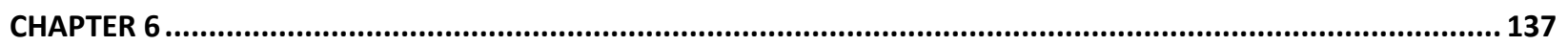

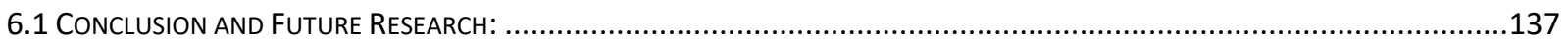

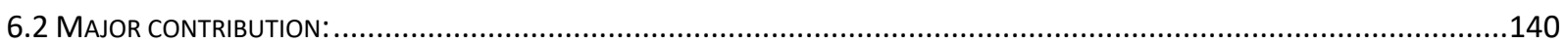

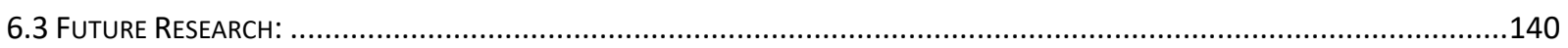

6.3.1 In-vitro cancer detection of cellular components using label-free nickel nanomaterial: .....................141

6.3.2 Examine nickel nanonetwork influence in Co-Cultured In-Vitro environment: ................................141

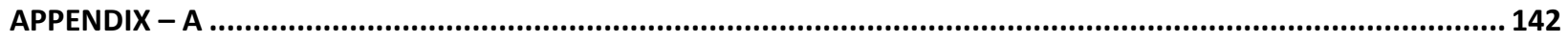

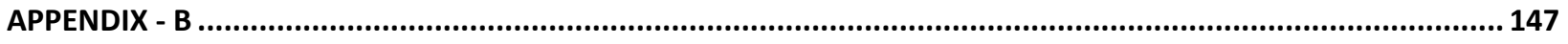

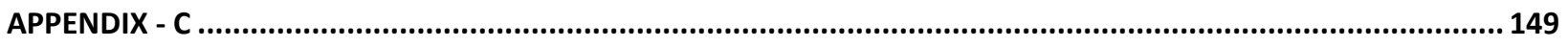

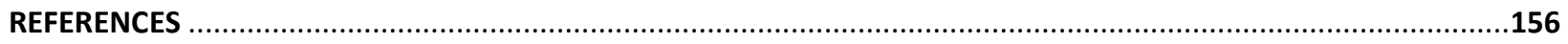




\section{LIST OF TABLES}

Table C S1: Comparison of laser parameters with synthesized nanonetwork nomenclature 150

Table C S2: Raman excitation comparison based on nanonetwork composition vs GSH dye concentration.............. 154

Table C S3: A comparison of the proposed method with other methods for biomolecule detection.......................... 154

Table C S4: A comparison of the proposed SERS based nano-biosensor with other SERS biosensors ......................... 154

Table C S5: Laser parameters with corresponding synthesized nanonetwork image and Energy

Dispersive X-ray image of observed nanonetwork

155 


\section{LIST OF FIGURES}

Figure 2-0: Overall schematic of nanomatrix formation using multiphoton ionization mechanism and subsequent drug-free cancer therapy 14

Figure 2-1: Self-assembled nickel nanomatrix formation through femtosecond pulse laser interaction mechanism achieved by programming ultra-short femtosecond laser pulses to ionize nickel

Figure 2-2: Nanoparticle morphology distribution and its Raman activity. Figure 2A(1-3) Summary of nanomatrix morphological distribution. All the images have a uniform scale of $20 \mathrm{~nm} \mathrm{~B}$ ) Raman activity on native and nickel oxide nanomatrix generated at corresponding laser ionization energies

Figure 2-3: X-Ray Diffraction and Reitveld analysis of the synthesized nanomatrix. Figure 3A) XRD spectrum obtained for synthesized nanomatrix B) Elemental Reitveld fitting of observed spectrum is fitted using Pie chart showing the weight percentage of observed phases 22

Figure 2-4: Quantifying Lactate dehydrogenase leakage (LDH) by nickel oxide nanomatrix in HeLa and NIH3T3 fibroblast cells. Figure 4 (A) Interval plot determining the LDH release in all NiO nanomatrix for HeLa and NIH3T3 cell in 24 hours, (B) Interval plot determining the LDH release in all NiO nanomatrix for HeLa and NIH3T3 cells in 48 hours

Figure 2-5: Nanomatrix interaction with HeLa and NIH3T3 fibroblast cells. HeLa cells present on nanomatrix show circular morphology (selective apoptosis phase) when comparing with NIH3T3 fibroblast cells 27

Figure 2-6: Quantifying HeLa and NIH3T3 Fibroblast cell proliferation on synthesized nanomatrix. A comparison between HeLa and NIH3T3 fibroblast cell interaction on synthesized nanomatrix and unprocessed native nickel control surface for 24 hours and 48 hours. (A-H) fabricated platforms and (I-P) unprocessed native nickel for 24 to 48-hour incubation. $Q$ and R) Graphical representation of HeLa and NIH3T3 fibroblast cells on nanomatrix interaction after 24 and 48 hours as compared to control native nickel substrate All the fluroscence images have a uniform scale of $10 \mu \mathrm{m}$

Figure 2- 7: Influence of different nickel nanomatrix on adhesion (24 hours) and proliferation phase (48 hours).

A) Comparison between the $67.29 \% \mathrm{NiO}$ and $100 \% \mathrm{NiO}$ nanomatrix platforms over HeLa cell adhesion. B) Comparison between the $67.29 \% \mathrm{NiO}$ and $100 \% \mathrm{NiO}$ nanomatrix platforms over NIH3T3 fibroblast adhesion. A uniform scale bar of 10 and $3 \mu \mathrm{m}$ was followed. The green indicators are filopodial presence and their interaction on nanomatrix. 32

Figure 3-1: Characterization of primary (quantum $\mathrm{Ni}$ ) and secondary (quantum $\mathrm{NiO}$ ) theranosomes. The FESEM shows the self-assembled 3D quantum structures. The HR-SEM insets shows individual particle morphology which are composed of primarily spherical and cubic particles. HR-TEM displays lattice spacing 'd' indicating the presence of Nickel and Nickel oxide along with particle size distribution

Figure 3-2: A) Raman spectra of primary (quantum-Ni) and secondary (quantum-NiO) theranosomes B) X-Ray diffraction and reitvield analysis of the synthesized theranosomes to qualitatively identify weight percentage of observed phases 55

Figure 3-3: XPS survey and individual spectrum exhibiting strong presence of nickel and oxygen at A) Primary Theranosomes and B) Secondary Theranosomes C) UV-Vis spectra of theranosomes exhibiting increased photon absorption D) Room temperature electron paramagnetic resonance curves for different theranosomes 57

Figure 3-4: Emission of theranosomes at excitation wavelengths of multiple channels at 358, 494,577 nm exhibits the fluorescence characteristics of the theranosomes using flow cytometry. A comparison fluorescence intensity at A) primary theranosomes and B) secondary theranosomes C) A representative comparison shows the different fluorescence intensity of the synthesized colloidal theranosome particles under corresponding fluorescence excitation channels in fluorescence microscopy. Photoluminescence $(P L)$ synchronous spectrum of D) Primary and E) Secondary theranosomes showed strong PL with respect to the excitation wavelength. The emission was at a broadband visible wavelength which demonstrates fluorescence excitation of theranosomes 59 
Figure 3-5A: Representative TEM image of Primary theranosomes in HeLa, MDAMB-231 and NI3T3 fibroblast cells. The theranosomes are located within the cellular membrane in both cancer cells when compared with fibroblast cells. The corresponding Dark-field images on the right show the location and localization of theranosomes 61

Figure 3-5B: Representative TEM image of Secondary theranosomes in HeLa, MDAMB-231 and NIH3T3 fibroblast cells. The theranosomes are located within the cellular membrane in both cancer cells when compared with fibroblast cells. The corresponding Dark-field images on the right show the location and localization of theranosomes

Figure 3-6A: Primary Theranosomes acting as cancer detector demonstrating increased fluorescence intensity of cancer (MDAMB-231, HeLa) cells when compared with fibroblast (NIH3T3) cells. A standard scale of $10 \mu \mathrm{m}$ and $50 \mu \mathrm{m}$ for insets are deployed 64

Figure 3-6B: Secondary Theranosomes acting as cancer differentiator demonstrating increased fluorescence intensity of cancer (MDAMB-231) cells when compared with both fibroblast (NIH3T3) and HeLa cancer cells. A standard scale of $\mathbf{1 0} \mu \mathrm{m}$ and $50 \mu \mathrm{m}$ for insets are deployed 65

Figure 3-7: Cellular proliferation of NIH3T3 fibroblast, HeLa and MDAM-231 cancer cells on primary and secondary theranosomes. (a) Representative SEM \& fluorescence images showing the proliferation of three different cell lines, metastatic MDA-MB-231, and HeLa cancer cells, as well as the noncancerous NIH3T3 fibroblast cells

Figure 3-8: Influence of primary and secondary theranosomes on adhesion (24 hours) and proliferation phase (48 hours) A) Comparison between the quantum theranosomes over Hela cell adhesion B) Comparison between the quantum theranosomes over MDAMB cell adhesion. adhesion C) quantum theranosomes over NIH3T3 cell adhesion. A uniform scale bar of 10 and $3 \mu \mathrm{m}$ was followed. The red indicators are filopodial presence and their interaction on theranosomes 69

Figure 3-9: ROS generation- DCFH-DA assay. The dot plots in blue color indicate the cells under ROS through increased fluorescence intensity with respect to control. A) NIH3T3 and MDAMB-231 cells treated with primary B) secondary theranosomes (C) graph showing the percentage of cells in showing DCF fluorescence. The data represents mean \pm SD of three independent experiments

71

Figure 4-0: Overall schematics of SERS-active nanobiosensor synthesis and subsquent biomolecule interaction 84

Figure 4-1: Functionalized 3D hybrid nickel nanonetwork fabrication on nickel biochip with dual wavelength Raman activation for GSH and CV detection 88

Figure 4-2: Synthesis of 3D nickel nanonetwork on nickel substrate using multi-photon ionization mechanism and subsequent Raman self-activation. The multiphoton ionization created cuboidal, spheroidal-cuboidal and spheroidal morphology nanonetwork 90

Figure 4-3: SERS activated nickel nanonetwork at cuboidal, spheroidal-cuboidal and spheroidal nanonetwork morphology along with inactive nickel substrate at A) $532 \mathrm{~nm}$ Raman laser excitation B) 785 nm Raman laser excitation and C) compositional analysis of corresponding nickel nanonetworks

Figure 4-4: Functionalized Raman activation of synthesized nanonetwork with crystal violet from millimolar to picomolar concentration for $532 \mathrm{~nm}$ Raman laser irradiation at A) Cuboidal nanonetwork B) SpheroidalCuboidal nanonetwork C) Spheroidal nanonetwork 96

Figure 4-5: Functionalized Raman activation of synthesized nanonetwork with glutathione (GSH) biomolecule from millimolar to picomolar concentration for $532 \mathrm{~nm}$ Raman laser irradiation at A) Cuboidal nanonetwork B) Spheroidal-Cuboidal nanonetwork C) Spheroidal nanonetwork at $532 \mathrm{~nm}$ Raman laser irradiation _ 98

Figure 4-6: Limit of detection (LOD) established for Raman intensity plots of activated 3D hybrid nickel nanonetwork with different concentration profiles of A) Crystal Violet (CV) and B) Glutathione (GSH). Enhancement Factor (EF) of C) CV molecules at $1600 \mathrm{~cm}^{-1}$ and D) GSH molecules at $3300 \mathrm{~cm}^{-1}$ for different concentration profiles. 101

Figure 4-7: Functionalized Raman activation of synthesized nanonetwork with L-Cysteine (L-Cys) biomolecule for $532 \mathrm{~nm}$ Raman laser irradiation for Cuboidal nanonetwork, Spheroidal-Cuboidal nanonetwork and Spheroidal nanonetwork A) $1 \mathrm{mM}$ and B) $1 \mu \mathrm{M}$ concentration at $532 \mathrm{~nm}$ Raman laser irradiation. 102 
Figure 4-8: Functionalized Raman activation of synthesized nanonetwork with L-Methionine (L-Met) biomolecule for $532 \mathrm{~nm}$ Raman laser irradiation for Cuboidal nanonetwork, Spheroidal-Cuboidal nanonetwork and Spheroidal nanonetwork A) $1 \mathrm{mM}$ and B) $1 \mu \mathrm{M}$ concentration at $532 \mathrm{~nm}$ Raman laser irradiation. 104

Figure 4-9: Functionalized Raman activation of synthesized nanonetwork Cuboidal nanonetwork, SpheroidalCuboidal nanonetwork and Spheroidal nanonetwork at A) complex biological environment (Cell culture medium) and B) $1 \mu \mathrm{m}$ concentration of GSH added to complex biological environment (Cell culture medium) at $532 \mathrm{~nm}$ Raman laser irradiation 105

Figure 5-1: Multiphoton ionization mechanism to generate quantum probes with atomic scale antennas _ 119

Figure 5-2: Portable quantum probes with atomic antennas morphological and size distribution ___ 120

Figure 5-3: Raman spectrum of portable quantum probes with atomic antennas _ 121

Figure 5-4A: Quantum probe interaction at picomolar, femtomolar concentration of CV__ 123

Figure 5-4B: Quantum probe interaction at picomolar, femtomolar concentration of R6G _ 124

Figure 5-5: Quantum probes interaction at nanomolar concentration of BSA along with enhancement factor relationship for crystal violet and rhodamine $6 \mathrm{G}$ 126

Figure 5-6: Quantum probe uptake in cells validated through TEM after 24 hours incubation and its corresponding Raman response A) HeLa B) Breast cancer C) Fibroblast cells 127

Figure 5-7: Detection of intracellular protein of cells in the presence of quantum probes. The peak positions at $1397 \mathrm{~cm}-1$ in combination with $1584 \mathrm{~cm}-1$ is identified as phenylalanine and tryptophan. The representative TEM images of HeLa and Fibroblast cells are showcased to verify quantum probe uptake in them. The location these probes is identified using black circular outline in TEM images 129

Figure 5-8: Detection of intracellular DNA and RNA of cells in the presence of quantum probes. The peak at 810 cm-1 is identified as RNA outlined in black. The peak identified at $1066 \mathrm{~cm}-1$ is identified as DNA outlined in orange. The location of quantum probes is identified using black circular outline in the TEM images 131

Figure 5-9: In-vitro Raman response to Quantum probes interaction with HeLa and breast cancer cells over 6,12 and 24 hours. The cytochrome $c$ peak positions are boxed in black outline. The corresponding TEM images show the quantum probes uptake in both cancer cells after 24 hours 


\section{LIST OF APPENDICES}

Figure A-S1: Quantifying HeLa and NIH3T3 fibroblast cell proliferation on synthesized nanomatrix for 24 hours and 48 hours. (A-D, E-H, I-L, M-P) fabricated platforms. Images (B, D, F, H, J, L, N, P) have a uniform scale of 10 $\mu \mathrm{m}$ while the rest has a uniform scale of $100 \mu \mathrm{m}$.

143

Figure A-S2: Nanoparticle in $\mathbf{1 0 0} \%$ NiO nanomatrix interaction with HeLa and NIH3T3 fibroblast cells. SEM image and EDX elemental mapping of oxygen (green), Nickel (Red) and Oxygen (grey). All the images have a uniform scale of $100 \mu \mathrm{m}$ 144

Figure A-S3: Elemental Energy Dispersive X-ray (EDX) mapping on nanoparticle interaction with cells at $67.29 \%$ NiO nanomatrix A) HeLa and B) NIH3T3 cells. A) Nanoparticle in $67.29 \% \mathrm{NiO}$ nanomatrix interaction with HeLa and NIH3T3 fibroblast cells. SEM image and EDX elemental mapping of oxygen (green), Nickel (Red) and Oxygen (grey). All the images have a uniform scale of $100 \mu \mathrm{m}$. B) Nanoparticle in $100 \%$ NiO nanomatrix interaction with HeLa and NIH3T3 fibroblast cells. SEM image and EDX elemental mapping of oxygen (green), Nickel (Red) and Oxygen (grey). All the images have a uniform scale of $100 \mu \mathrm{m}$ 146

Figure B-S1: White Light Emission at Primary and Secondary Theranosomes along with a representative broadband emission 147

Figure B-S2: Fluorescence Intensity of NIH3T3, HeLa and MDAMB- 231 cells upon native controls. All the images are set to a standard scale of $10 \mu \mathrm{m}$. 147

Figure B-S3: Stained and unstained control for ROS and Apoptosis assessment. 148

Figure C-S1(A) AFM image of height and phase analysis on synthesized nanonetwork along with inactive native nickel substrate. A uniform scale bar is $100 \mu \mathrm{m}$ is followed. B) Reitveld refinement of synthesized nanonetwork. C) XRD pattern of at cuboidal and spheroidal nanonetwork. 151

Figure C-S2: Activated nickel nanonetwork in nickel biochip interaction with Crystal Violet (CV) at dual (532 $\mathrm{nm}$ ) $\&(785 \mathrm{~nm})$ Raman wavelength 152

Figure C-S3: Activated nickel nanonetwork in nickel biochip interaction with biomolecule Glutathione (GSH) at dual (532 nm) \& (785 nm) Raman wavelength 153 


\section{LIST OF ABBREVIATIONS}

3D - Three-Dimensional

AFM - Atomic Force Microscopy

CV - Cyrstal violet

Cys - L-Cysteine

DMSO - Dimethyl Sulfoxide

ECM - Extracellular Matrix

EDX - Energy Dispersive X-ray

EF - Enhancement Factor

GSH - L-Glutathione

HR-TEM - High resolution Transmission Electron Microscopy

LOD - limit of detection

Met - L-Methionine

MHz - Megahertz

MRI - Magnetic Resonance Imaging

$\mathrm{Ni}-\mathrm{Nickel}$

NIR - near-infrared

SEM - Scanning Electron Microscope

SERS - Surface Enhanced Raman Scattering

SPR - Surface Plasmon resonance

TEM - Transmission Electron Microscope

XPS - X-ray spectroscopy

XRD - X-ray diffraction 


\section{LIST OF NOMENCLATURE}

Apoptosis - Death of a cell in a normal, controlled fashion

Biocompatible - Characteristic of not being harmful to tissue

Cytoskeleton - A network of protein filaments that give shape to a cell

Enhancement Factor - Raman Enhancement of chemical when compared with Raman enhancement of native substrate

Extracellular matrix - A collection of molecules that are secreted by cells that provides structural and biochemical support to the cells

Fibroblasts - A type of cell that is critical in wound healing

Filopodia - Slender cytoplasmic projections that aid in cell migration

Focal Adhesion - Strong adhesion points by the cell to the substrate

Integrin - Receptors that form bridges between cell-cell and cell-substrate interactions

In-Vitro - Tests that takes place outside a living organism

In-Vivo - Tests that take place inside a living organism

Lamellipodia - Flattened protrusion of the cell that adheres to the substrate

Nucleus - Dense organelle in a cell that contains the genetic material of the cell

Proliferation- Rapid increase in numbers

Theranostics - Simultaneous detection and treatment of cancer

Theranosomes - Synthesized quantum nickel particles exhibiting fluorescence and cancer therapy 


\section{CHAPTER 1}

\section{INTRODUCTION}

Nanoparticles in the $(1-100 \mathrm{~nm})$ range have currently gained increased research interest due to their nanodimension, unique physical and chemical characteristics ${ }^{1}$. At present, nanostructured metal and their metal oxides are of interest and have achieved prominence with great potential for both commercial and industrial applications. In recent years, there is an emerging interest to synthesize nanoparticles of Iron $(\mathrm{Fe})$, Cobalt $(\mathrm{Co})$ and Nickel $(\mathrm{Ni})$ due to their superior properties and versatile application in sensing, memory storage, photocatalytic, drug delivery and magnetic resonance imaging ${ }^{2,3}$. In the recent past, nanoscale Nickel and Nickel oxide displayed their novel structural and optical characteristics offering an entirely new spectrum to be tapped for biomedical applications. A wide range of methods have so far been applied for the synthesis of these nanoscale nickel and nickel oxide, using sol-gel, co-precipitation, solvothermal, hydrothermal, microwave irradiation and laser irradiation techniques ${ }^{4-10}$. The aforementioned techniques helped to realize nanoparticles in a variety of shapes and structures such as spheres, cubes, nanosheets, core-shell, cauliflower shaped particles with size ranging between $10 \mathrm{~nm}$ to almost $6 \mu \mathrm{m}$.

\subsection{NICKEL BASED NANOMATERIAL FOR BIOMEDICAL APPLICATIONS:}

Albeit, offering unique physical and chemical properties, the use of nickel based nanomaterials for biomedical applications such as biomedicine and biosensing are limited owing to crucial factors like nanoparticle toxicity, chemical composition, shape and surface charge ${ }^{11}$. Some studies have shown that a low level of $\mathrm{Ni}$ is essential for normal cellular development, as Ni deficiency reduces iron content in organs and haemoglobin ${ }^{12}$. In-addition, $\mathrm{Ni}$ has several biological functions, including transmission of genetic codes (RNA, DNA) and transport of oxygen in biological systems ${ }^{13}$. In the realm of nano-biotechnology, 
utilization of suspended nanoparticles opens new exciting developments in the field of nanomedicine and theranostics (cancer diagnosis and therapy). For instance, nanoparticles have shown the capability of achieving targeted cancer therapy which presents an alternative to the conventional and traditional chemotherapeutic strategy normally adopted. In addition to the conventional therapeutic function of nanoparticle now being employed, nanoparticle aided imaging is currently gaining momentum as a promising diagnostic application tool.

\subsection{CONVENTIONAL NANOMATERIALS FOR CANCER TREATMENT:}

Nanomaterials that are currently investigated for cancer treatment use conventional cytotoxic agents or

drugs to induce cancer treatment. These cytotoxic agents do not demonstrate the specific applicability to tumor cells when compared with normal cells and thereby rely upon favourable nanoparticle bio distribution mechanism to achieve the desired cancer treatment. During the cancer treatment process, they eventually tend to cause cytotoxic response to the surrounding normal cells present along with the cancer cells cluster. This is due to the inability of nanoparticles to discriminate normal healthy cells from those of the cancerous cells. One promising approach to achieve the desired target fixing is by employing magnetic responsive nanoparticles such as $\mathrm{Ni}, \mathrm{Fe}, \mathrm{Co}$, Gadolinium $(\mathrm{Gd})$ which can be aimed at using both internally and externally targeting sources such as magnetic resonance and alternating magnetic field to achieve the specified and localized cancer treatment. These techniques play a vital role in introducing drug delivery systems to achieve cancer treatment demonstrating superior performance compared to the traditional cytotoxic drugs.

Although, these nanoparticles support the process by increased drug delivery, however the significant drawback noted being that of the nanoparticles total degradation due to non-preferential heating leads to premature drug release necessitating the need for a constant field for cancer treatment. This more often presents a reduced specific cancer cell interactions, leading to nanoparticle internalization thereby 
causing poor biocompatibility as they cannot distinguish between cancerous and healthy cells ${ }^{14}$. Hence, a novel approach is necessary to precisely create a biocompatible nanomaterial to achieve cancer treatment without the need of any external targeting agents. This is achieved using a biocompatible nickel nanomatrix synthesized using ultra-short pulsed laser which modulates cancer treatment (as given in chapter 3).

\subsection{THERANOSTICS APPROACH USING NICKEL FOR CANCER TREATMENT AND BIOIMAGING:}

To create a viable theranostics agent, the nanoparticles that induce cancer treatment should also be used for prognosis of cancer. This can be done using non-invasive optical imaging techniques such as fluorescence and two photon imaging ${ }^{15}$. Since the nanoparticles do not inherit fluorescence excitation, they are currently tagged with traditional fluorescence probes to accomplish bioimaging. However, conventional biological imaging utilizes organic fluorophores which suffer major drawbacks due to nonspecific fluorophore accumulation, short life time (ns to $\mu \mathrm{s})$, limited contrast features and photobleaching. Hence there is an inherent need for a diagnostic probe that overcomes all the aforesaid limitations encountered using conventional organic fluorescent probes.

Some successful investigations have reported the feasibility of using semiconductor and metal quantum dots (QD) or quantum scale (QS) materials as a substitute to organic fluorescent dyes. The unique characteristics of these QDs is that they exhibit a specific wavelength upon photon absorption. The QDs exhibit different color primarily due to its size, ranging from 2 to $10 \mathrm{~nm}$ owing to the quantum confinement effect. These QDs share dimensional similarities with biological elements present such as proteins and nucleic acids. This feature makes them as an ideal choice for fluorescence imaging. But, the use of QDs for bioimaging applications are severely restricted due to cytotoxicity, particle size dependency for fluorescence excitation and bioconjugation labels for in-vitro application to induce fluorescence excitation ${ }^{16,17}$. Therefore, there is still a very significant vacuum and need for a biocompatible 
label-free QS bioimaging probe which could incidentally also induce cancer treatment. Nickel theranosomes presented in this research demonstrated that the sub-nanoscale (4 to $8 \mathrm{~nm}$ ) nickel and nickel oxide particles can be very much used not only for cancer therapy but also used for fluorescence based bioimaging probes while investigating for cancer treatment. Therefore, simultaneous benefits of cancer therapy and diagnostics (i.e., theranostics) can be achieved by fine tuning the nickel nanoparticles both in terms of its physical and chemical properties.

\subsection{NICKEL FOR BIOSENSING SENSING APPLICATION:}

In-addition to the development of fluorescence bioimaging probes for cancer theranostics, the need for ultrasensitive biosensing probes for bio-analytes (biomolecules) lack certain niche thereby restricting its use for biosensing applications. A significant challenge overcome in bioanalytical sensing is the development of sensitive platform that supersedes the limitations of conventional optical sensing techniques which includes UV-Vis spectroscopy, resonance elastic light scattering, fiber optics sensing and spectrofluorometric sensing. This development of the platform has gained much attention in biomedical science due to their increased sensitivity, improved spatial resolution, and throughput capability ${ }^{18-20}$. Among these, Surface Enhanced Raman Scattering (SERS) is most valuable as it tackles the current limitations of fluorescence imaging by achieving higher sensitivity and with broad excitation wavelength. In-addition, it offers unique molecular fingerprint data of the given analyte with limited photobleaching, reduced background interference and auto fluorescence. To date, primarily only noble metal and noble metal coated nanomaterial have been used for analyte detection with the possibility of up to single molecule detection ${ }^{21,22}$. Nevertheless, the usage of all these noble metals is severely restricted due to lack of biocompatibility. The desire to have the way forward for utilizing these nanostructures in SERS technique applications for chemical sensing using ultrashort pulsed laser had already been established earlier in our lab by Maznicheko et al., and Powell et al., using titanium and silicon ${ }^{23,24}$. However, their 
current state is very much limited to chemical analytes like crystal violet (CV) and Rhodamine (6G). We herein demonstrated (as given in chapter 4) an ultrasensitive nano biosensor using nickel for both chemical molecule (CV) and biomolecule glutathione (GSH) application. The GSH biomolecule was sensed in a complex biological medium only to showcase and explicitly demonstrate the nano-biosensor selectivity property.

\subsection{Nickel QuANTUM PROBES TOWARDS CANCER DIAGNosis:}

SERS offered a powerful tool for biological analysis presenting rapid non-destructive technique to analyse in-vitro and in-vivo sensing. Live cell SERS analysis yields useful biochemical information pertaining to cells primarily to identify and differentiate diseases, toxic agents and other various factors. Even though conventional SERS employ noble metal hotspots and noble metal decorated nanoparticles to achieve single molecule sensitivity, the issue of biocompatibility, Raman wavelength excitation and selectivity were not addressed. Earlier in chapter4, we demonstrated the SERS active nano biosensor to selectively identify and enhance GSH biomolecule sensitivity. Upon successful demonstration of applicability of nickel nano biosensor in this dissertation, we explored the possibility of also using nickel quantum probes for in-vitro SERS for cancer diagnosis. The unique characteristics of these Nickel quantum probes relies on both localized surface plasmon resonance (LSPR) and material chemistry introduced through quantum confinement effect.

\subsection{SUMMARY}

The current application of nanoparticles in the field of nanomedicine is limited to either as a carrier for drug delivery or as a fluorescent probe carrier for diagnostic imaging. The nanomaterials can be fine tuned to act and induce the same effect as that of a drug and the same time to mimic as a bioimaging probe. Since there are very limited researches and studies currently available encompassing this issue, the 
current thesis is aimed basically to investigate the possibility of utilizing Ni-based nanomaterials for cancer theranostics (cancer therapy and diagnostics) applications.

The contents of this thesis are aimed to take advantage of multidisciplinary approach that can be undertaken by combining cancer therapy, fluorescence bioimaging and Raman spectroscopy so as to offer a very comprehensive single step solution as a drug-free cancer therapy and diagnostics.

\subsection{Research Objectives:}

Ni-based nanostructures generated through ultrashort pulsed laser ionization offer promise not only as biosensor but also as nanomedicine. This fabrication technique generates nanostructures with network and web like morphology that could be easily be fine-tuned to modify the material chemistry of the nickel oxide within the nanostructure. This excellent feature of extending the control to redefine the physical morphology of the structure by deployment of the laser-ion fabrication technique allows for both the precise but also an optimized solution in cancer theranostics.

The main objective of this research is to study and explore the possibility of creating self-assembled nickel nanonetwork and to determine as to how this material can be used for biomedical sensing and cancer theranostics application.

\section{Determine the cell-material interaction and modulating cellular function using NiO nanomatrix:}

The first step in this process is to engineer, fabricate and generate a 3D nanomatrix structure by varying multiphoton ionization laser characteristics. Subsequently, evaluate cellular adhesion, proliferation and cancer cell apoptosis characteristics when they are interacting with the synthesized nanomatrix. To identify and evaluate the synthesized nanomatrix platform mimicking ECM characteristics to derive a drug-free cancer therapy. 
Determine the cancerous and mammalian fibroblast cell interaction on quantum scale theranosomes for cancer therapy and fluorescence bio-imaging for cancer theranostics:

Fabricate a quantum scale material using multiphoton ionization mechanism process by exploring the laser ionization plume characteristics variation with the introduction of ionizing gases species to create a variety of $3 \mathrm{D}$ quantum scale theranosomes. Thereafter, assess the synthesized quantum scale material for their physical and chemical properties and finally determine their cellular viability for cancer therapeutic and bioimaging activity for cancer theranostics.

Create a SERS active nickel nanonetwork using multiphoton laser ionization for biosensing application: Having narrowed down on to the desired nickel nanonetwork, we determined the Raman activeness of the synthesized nanonetwork using SERS for molecular sensitivity. Furthermore, Crystal Violet (CV) and glutathione (GSH) were both used to evaluate SERS response of the synthesized nanonetwork for biosensing application.

Generate Raman active atomic antennas on quantum structures on in-vitro cancer diagnosis:

Assessing the Raman activity of quantum probes using established molecular sensitivity and diagnosis and differentiate the cancer cells from fibroblast cells using in-vitro Raman sensing. Examine the cellular viability and determine Raman activity in distinguishing and diagnosing cancer cells.

\subsection{ORGANIZATION OF DISSERTATION}

This thesis has been organized as a manuscript based manner. It describes the synthesis of nickel nanostructures using multiphoton ionization mechanism through ultrashort pulsed laser. The following is the list of manuscripts in peer-review and also already published in scientific journals as presented in each chapter.

Chapter -1 Present review of current research works pertaining to use of nanomaterials for theranostics 
Chapter-2 Presents the role of nickel nanomaterials has on cell behaviour. The concept of 3D nanomaterial morphology combined with precise nanomaterial material chemistry induced cell selective cancer apoptosis. The findings revealed that the nickel oxide ( $\mathrm{NiO}$ ) mimicked ECM like behaviour and induced HeLa cancer cell apoptosis while simultaneously increasing mammalian fibroblast cells. This demonstrates the biocompatibility of the synthesized nickel oxide which is a fundamental criterion for the evolution a drug-free approach towards cancer therapy.

Chapter-3 Introduces the concept of label-free bioimaging combined with cancer therapy (theranostics) through quantum theranosomes. The quantum (primary and secondary) theranosomes exhibited cellselective uptake that allowed differentiation and diagnosis of cancer (HeLa \& MDAMB-231) and fibroblast cells. In-addition, the synthesized theranosomes showed supremacy in inducing cancer cell apoptosis with pronounced ROS generation. The results directly implied the idea of possible and definitive usage of quantum theranosomes leading to an entirely new pathway for cancer theranostics.

Chapter-4 Delineates the synthesis and SERS-activation of nanonetwork structure for biosensing application. The nanonetwork enhanced the Raman response which aided in detection of chemical and biomolecules such as glutathione and crystal violet. In-addition, the Raman active biosensor demonstrated picomolar sensitivity of both chemical analyte and GSH biomolecule. This is the first time a "non-noble metal" based ultra-sensitive nano-biosensor platform was demonstrated at both $532 \mathrm{~nm}$ and $785 \mathrm{~nm}$ excitation level. This approach is promising for both in-vitro and in-vivo sensing applications.

Chapter-5 Introduces the concept of synthesis of Raman-activated quantum nickel probes for cancer diagnosis. The synthesized nickel probes with atomic antennas enhance the Raman spectrum of cellular components of both cancerous and fibroblast cells. The current study shows the potential capability of quantum nickel probes to monitor the chemical changes in live cells too. The results gave new insight to both differentiation and diagnosis process adopted for cancer cells. 


\subsection{STATEMENT OF CO-AUTHORSHIP}

The following people and institutions contributed to the publications of the work undertaken as part of this thesis:

Sivaprasad Chinnakkannu Vijaykumar, Ryerson University = Candidate

Dr. Krishnan Venkatakrishnan, Ryerson University, Thesis supervisor = Author 1

Dr. Bo Tan, Ryerson University, Thesis supervisor $=$ Author 2

Author details and their roles:

Paper 1, Selective Drug-free Cancer Apoptosis by Three-Dimensional Self-Targeting Magnetic Nickel oxide Nanomatrix Located in chapter 2. Candidate was the primary author who performed the lab work, collected the data and wrote the article. Authors 1 and 2 helped with its idea, drafting the work and revising it critically for publication.

Paper 2, 3D Quantum theranosomes: a new direction for label-free theranostics located in chapter 3. Candidate was the primary author who performed the lab work, data collection and wrote the article. Authors 1 and 2 helped with its idea, drafting the work and revising it critically for publication.

Paper 3, SERS active nano-biosensor functionalized by self-assembled 3D nickel nanonetworks for glutathione detection located in chapter 4 . Candidate was the primary author who performed the lab work, data analysis and writing. Author 1 and 2 contributed to the idea, its formalization and development.

Paper 4, Atomic antennas on portable quantum structures - A non TERS approach located in chapter 5. Candidate was the primary author who performed the lab work, data analysis and writing. Author 1 and 2 contributed to the idea, its formalization and development. 


\section{CHAPTER 2}

\section{Selective Drug-free Cancer Apoptosis by Three-Dimensional Self-Targeting Magnetic NICKEL OXIDE NANOMATRIX}

We demonstrate for the first time, a successfully synthesized 3D self-assembled magnetic nanomatrix of Nickel Oxide generated by femtosecond pulsed laser interaction now deployed as an alternative for cancer therapy without any need of an external agent. This nanomatrix mimicked Extra Cellular Matrix (ECM) resulting from the synergistic effect of $\mathrm{NiO}$ in terms of physical configuration and its properties. Comparative cellular interaction results of both HeLa and NIH3T3 cells independently revealed their direct effects on cellular adhesion, proliferation and cytotoxicity. The bifunctional property in attracting cells and later acting as a drug to selectively kill HeLa cells was noticed. Accelerated proliferation of HeLa and NIH3T3 noticed during first 24 hours and a selective apoptosis upon HeLa cancer cells was observed after 48 hours.

\subsection{INTRODUCTION:}

Magnetic nanomaterials have received extensive attention for cancer therapy owing to its unique physiochemical properties, magnetic guidance and self-heating property when under an AC-magnetic field ${ }^{25,26}$. Magnetic nanomaterials have demonstrated superior performance compared to its organic counterparts $^{27-29}$. In the field of cancer theranostics research, numerous exciting developments with the use of magnetic nanomaterials have also been actively reported ${ }^{22}$. For instance, magnetic nanomaterials such as Nickel and Cobalt based nanoparticles are primarily researched for their unique functionality and drug loading capacity while Iron oxide based nanoparticles are studied in depth for its biocompatibility ${ }^{30-}$

32. At present, magnetic nanomaterials are used as drug carriers for targeted delivery ${ }^{33}$, as contrast agents 
for image based diagnosis ${ }^{34}$, and hyperthermia treatment using external targeting agents ${ }^{35,36}$. The multifunctionality of these magnetic nanomaterials enables a combined approach towards cancer therapy which allows integration of multiple diagnosis and treatment strategies both embedded into a single carrier system using external stimuli and biocompatible coatings ${ }^{37-39}$. The need for self-functionalized magnetic nanomaterials free of chemotherapeutic drugs for achieving targeted cancer therapy without external stimulus has never been reported before.

Till date, current approaches made towards cancer therapy using magnetic nanomaterials can be broadly divided into passive and active approaches. In the passive approach mode, magnetically active nanomaterials are loaded with chemotherapeutic drugs and guided to desired affected sites using an external magnetic field. Once the nanomaterial is delivered at their targeted site, the drug is unloaded and released to the surrounding tissue through a variety of simulation techniques such as $\mathrm{pH}$ modulation, photodynamic and thermal activation or a combination of these methods ${ }^{40,41}$. In the passive approach mode, the magnetic particles are simply used as a platform for delivery of drug and do not in any way directly participate in the cancer therapy. For instance, increased apoptotic hyperthermia is observed in skin (B16) cancer cells when Polyvinylpyrolidone (PVP) coated nickel nanoparticles are utilized. However, the efficiency of this treatment is dependent totally on the strength of the alternating magnetic field applied to the target area, nickel nanoparticle concentration and PVP coating thickness ${ }^{42}$. In the same way, Alexiou et al., targeted VX-2 squamous cell carcinoma by combining the chemotherapeutic agent Mitoxantrone (MOT) and superparamagnetic iron oxide $\mathrm{Fe}_{3} \mathrm{O}_{4}$ nanoparticles in the presence of external magnetic field. The aggregated iron oxide nanoparticles aided in increased drug delivery ${ }^{43}$. Nevertheless, the non-preferential heating due to magnetic field caused severe side effects as it relies totally on favorable bio-distribution and magnetic field penetration depth to achieve maximum impact from the chemotherapeutic drugs delivered ${ }^{44}$. Similarly, An et al., reported magnetically targeted GSH enzyme triggered release of DOX to HeLa cells using iron oxide in silicon dioxide core shell nanoparticles ${ }^{45}$. In all 
the above passive approach modes, a constant external magnetic field is necessary for guided drug delivery and the nanoparticles merely acts as a carrier agent.

In contrast to the passive approach mode, the active approach to cancer therapy was conceived using magnetic particles which serves multiple purposes. Apart from drug carrying property, these magnetic nanoparticles function as theranostics agents ${ }^{46}$, such as cell labelling biomarker ${ }^{47}$ and hyperthermia inducers $^{48}$. For instance, a $\mathrm{pH}$-response based magnetically targeted delivery of Doxorubicin hydrochloride (DOX) to HeLa cells using iron oxide in silicon dioxide core shell nanoparticles has been demonstrated ${ }^{49}$. However, the premature release of anticancer drug to the surrounding healthy cells pose a significant threat, thus restricting its use to inflammation and low-pH tumor sites. Thus, neither passive nor active approach can be used solely for cancer therapy. Hence, they are used in unison with other conventional anticancer drugs to achieve optimal therapeutic effect and subside the side effect to healthy tissues $^{50}$. Moreover, external electric and/or magnetic fields are very much required to excite nanoparticles to induce cytotoxic and apoptosis effect on cancerous cells. Therefore, there exists a real need for non-cytotoxic drug free magnetic nanomaterial to achieve superior anti-tumor response for cancer therapy.

Thus, recently much efforts have been devoted to the synthesis of nontoxic nickel based nanoparticles through green route ${ }^{51}$. On the contrary, nickel oxide nanoparticles were also proposed as anticancer drug due of its toxicity ${ }^{52}$. However, this approach is of very little practical application as it does not discriminate healthy cells from cancerous cells. In addition to the drug free property, current magnetic nanomaterials also lack self-targeting phenomenon to achieve a targeted reaction for cancer therapy. This is primarily due to their lack of structure that imitates 3D extra cellular matrix (ECM). Currently, all materials used are 2D nanomaterials only. It is widely accepted that cancer growth in any environment is directly related to tumor microenvironment. This tumor microenvironment predominately consists of ECM, cancer cells and non-malignant cell types such as fibroblasts and mesenchymal stem cells. Thus, it is very important to 
achieve a structure that mimics 3D ECM and also inherit the property of a drug for therapeutic effect as they are the key factors to hinder the progress of metastatic cancer in both primary and secondary tumor sites $^{53-56}$.

Here, we propose to introduce the concept of a drug free nanomedicine for cancer therapy using magnetic NiO nanomatrix. In addition, we also successfully present the idea of synthesizing 3D magnetic nanomatrix mimicking ECM like structure by femtosecond pulse laser interaction mechanism which to the best our knowledge is never been reported before. Moreover, the synthesized magnetic nanomatrix has bifunctional property with nanomatrix chemistry acting not only as a drug to selectively kill cancer cells but the 3D self-assembled magnetic nanomatrix now acting as an ECM. The NiO nanomatrix proliferated fibroblast cells and immortal HeLa cancer cells after 24-hour incubation period mimicked the 3D architectural structure like that of ECM. The same nanomatrix later induced selective apoptosis towards HeLa cells after 48 hours while fibroblast cells continue to proliferate forming tissue-like structures with negligible toxicity through LDH leakage examination. This selective apoptosis induced by structured nanomatrix upon HeLa cells demonstrated the drug like tumor response. This 3D ECM like structure exhibited by the nanomatrix also introduced a self-targeting property by proliferating HeLa cells during the first 24-hour of incubation, followed by a time delayed apoptosis. We attribute that the observed phenomenon resulted from the synergistic effect of various degree of NiO concentration within nanomatrix is synthesized by femtosecond pulse laser interaction mechanism. We believe that this study opens the possibility of using nickel based magnetic nanomedicine for drug free cancer therapy. 


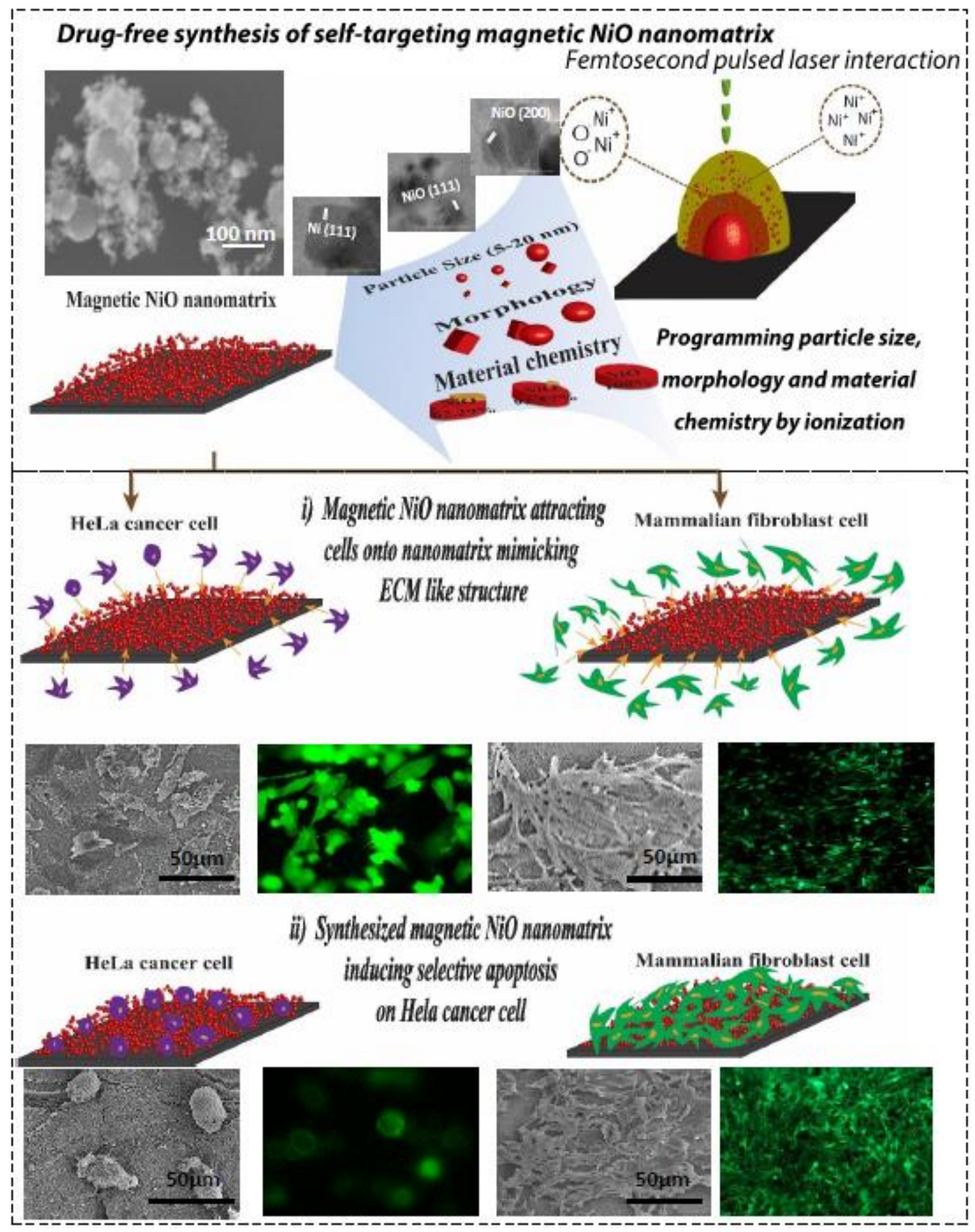

Figure 2-0: Overall schematic of nanomatrix formation using multiphoton ionization mechanism and subsequent drug-free cancer therapy 


\subsection{Materials AND Methods:}

\subsubsection{Materials:}

Nickel 200 Sheet purchased from (onlinemetals.com, USA) were cut into $(3 \times 10 \times 20)(D \times W \times L)$ dimensions having $99 \%$ nickel content is used for experimentation. Gradual mechanical polishing is achieved using SiC grit sandpaper (200 to 1200 ) on the surface and later ultrasonically cleaned in $100 \%$ acetone and ethanol for a 15-minute duration each to remove all embedded particle inclusions from polishing. The polished sample surface is washed with de-ionized (DI) water and consequently air dried for experimentation.

\subsubsection{Femtosecond pulse laser-material interaction:}

A tunable $\mathrm{Yb}$ doped diode pumped fiber laser from Impulse series (Clark - MXR) having a central wavelength of $1030 \mathrm{~nm}$ with an average power of 16 watts was employed for fabrication of nickel oxide nanomatrix. The laser beam having a Gaussian shaped beam profile was focused onto the nickel substrate with $10 \mu \mathrm{m}$ spot diameter. The femtosecond pulsed laser system employed has a varying laser pulse interaction time of $214 \mathrm{fs}$ to $1428 \mathrm{fs}$ with a laser pulse repetition rate between $200 \mathrm{KHz}$ to $25 \mathrm{MHz}$. The prepared nickel samples were mounted on an $x-y$ precision stage normal to laser beam. To maximize effectiveness of fabrication some of the processing parameters were kept constant, here the laser pulse interaction time was fixed at $214 \mathrm{fs}$ and average power at 16 watts. The nickel oxide nanomatrix was created over an array of lines with a uniform spacing of $250 \mu \mathrm{m}$ and different shape patterns to simulate tissue engineering and diagnostic device environments. The speed at which laser irradiates the nickel surface is maintained at $5 \mathrm{~mm} / \mathrm{s}$ respectively. EzCAD software combined with a two axis galvoscanner was used for plotting and fabrication. The nanomatrix was generated at laser fluence values of $5 \mathrm{~J} / \mathrm{cm}^{2}(4 \mathrm{MHz})$, $2.5 \mathrm{~J} / \mathrm{cm}^{2}(12 \mathrm{MHz}), 1.64 \mathrm{~J} / \mathrm{cm}^{2}(25 \mathrm{MHz})$. 


\subsubsection{Nanomatrix morphological and physiochemical characterization:}

The fabricated nanomatrix specimens were assessed using an environmental scanning electron microscope (ESEM; Quanta FEG 250). To evaluate and study laser pulse interaction with nickel, the fabricated nanomatrix were examined in high resolution scanning electron microscope (HRSEM). A carbon copper grid collected the nanomatrix and demagnetized them using a Neodymium magnet before examination. Then HRSEM EDX attachment from Oxford Instruments was used to primarily analyze the elemental composition of the laser synthesized nanomatix. To identify the oxide phase quantitatively and qualitatively, X-ray diffraction (Phillips powder XRD) was employed with Cu K- $\alpha$ radiation $(\lambda=1.54054 \AA ̊)$ at $40 \mathrm{KV}$ and $40 \mathrm{~mA}$ with a $2 \theta$ scanning speed of $0.010^{\circ} \mathrm{min}^{-1}$. Reitveld analysis was also carried out on the acquired spectrum. The synthesized nanomatrix were analyzed using Bruker Senterra Dispersive Raman Microscope fitted with a $532 \mathrm{~nm}$ laser system. A $50 \mathrm{X}$ objective was used to tightly focus the laser on the sample surface with appropriate laser irradiation power of $5 \mathrm{~mW}$. A standard collection time of $10 \mathrm{~s}$ with three iterations was employed and all the parameters were chosen based on earlier trial and error method to reduce spectrum noise. All spectral are baseline corrected and normalized to laser power.

\subsubsection{Cell Culture:}

HeLa, human cervical cancer cell line, was obtained from ATCC (American type culture collection, ATCC No. CCL-2) and were cultured in DMEM-F12 medium with phenol red containing $10 \%$ heat inactivated fetal bovine serum and $1 \%$ penicillin-streptomycin antibiotics at $37^{\circ} \mathrm{C}$ in $5 \% \mathrm{CO}_{2}$. NIH3T3, fibroblast cells also obtained from ATCC were grown in DMEM medium containing $10 \%$ heat activated fetal bovine serum with $1 \%$ penicillin-streptomycin antibiotics at $37{ }^{\circ} \mathrm{C}$ in $5 \% \mathrm{CO}_{2}$. The nanomatrix specimens are washed with alcohol and DI water and kept under UV light for 20 minutes. Subsequently, the substrates are placed in separate petri dishes with HeLa and NIH3T3 cells are seeded at a density of $10^{5}$ cells $/ \mathrm{ml}$, totaling to $4 \mathrm{ml}$ volume per dish. The petri dishes are placed in an incubator for 24 and 48 Hours. 
After the incubation period, the samples are fixed for SEM imaging in $2 \%$ glutaraldehyde in $0.1 \mathrm{M}$ sodium cacodylate buffer $\mathrm{pH} 7.3$ for one hour. Next, the samples are immersed in $0.1 \mathrm{M}$ sodium cacodylate buffer with $0.2 \mathrm{M}$ sucrose at $\mathrm{pH} 7.3$ for 20 minutes. The samples are dehydrated in increasing concentrations of alcohol for about 20 minutes each. The samples are then critical point dried. Imaging was conducted using (Hitachi SU 1500) at 10kV and magnification was varied between 500 and 15,000 times. Three separate images were chosen for each parameter to conduct quantitative analysis measuring cell populations.

\subsubsection{Fluorescent staining of cell:}

The samples are first fixed in $4 \%$ methanol free paraformaldehyde followed by incubation in $1 \%$ skim milk to prevent non-specific binding. To stain the actin cytoskeleton, the samples are incubated with Alexa fluor 488(Life Technologies) followed by DAPI (4', 6'-diamidino-2-phenylindole, Life Technologies) to stain the nucleus. The samples were air dried using an epi-fluorescent Nikon E-400 microscope was used and data were recorded by DS-5M-U1 color digital camera (Nikon, Canada).

\subsubsection{LDH assay:}

The LDH (Lactate dehydrogenase) test from Pierce LDH-cytotoxicity colorimetric assay kit was performed in accordance to the manufacturer's protocol. Samples in petri dishes were added with $1 \times 10^{5} \mathrm{cells} / \mathrm{ml}$ and allowed to incubate for $24 \& 48$ hours. After incubation, the absorbance at $490 \mathrm{~nm}$ and $680 \mathrm{~nm}$ is performed using a spectrofluorometer. To determine LDH activity the absorbance value at $680 \mathrm{~nm}$ is subtracted from $490 \mathrm{~nm}$ absorbance before calculation. The cytotoxicity then measured using the formula given below.

$$
\% \text { Cytotoxicity }=[(\text { LDH at } 490 \mathrm{~nm})-(\text { LDH at } 680 \mathrm{~nm})] \times 100
$$

The LDH activity in the collected culture media was taken as an indicator of damaged cells. Triplicate of each samples were made under the same conditions. The activity of cytosolic enzyme was then estimated 
by assessing the rate of NADH oxidation at $490 \mathrm{~nm}$ in the presence of pyruvate. The LDH levels in the culture medium versus those in the cells were quantified and compared with the control values.

\subsubsection{Statistics:}

All experiments are carried out in triplicates and data points are means \pm standard error unless otherwise mentioned. The error bars indicate standard deviation. A 95\% confidence interval for the mean is achieved. 


\subsection{Results AND Discussion:}

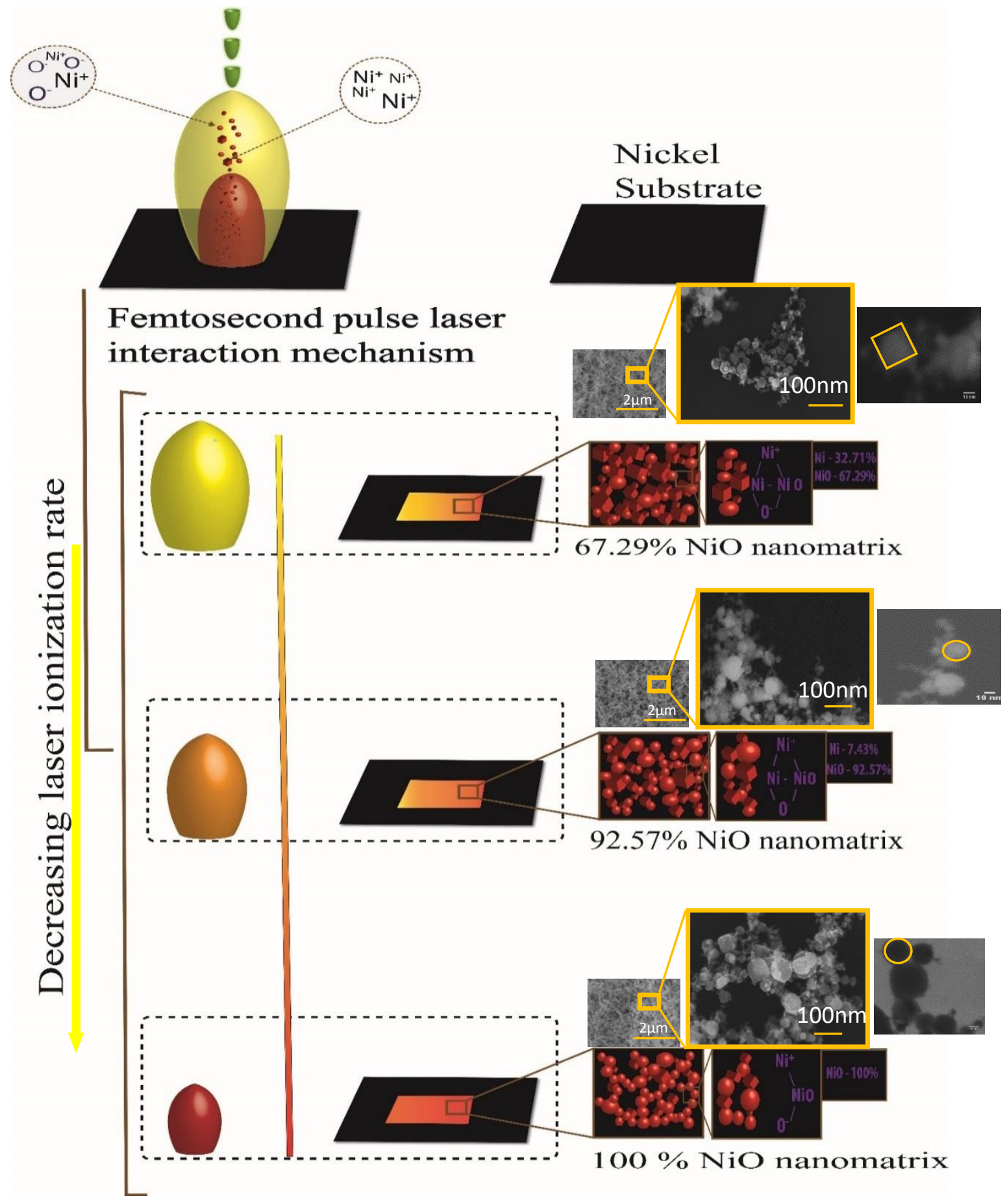

Figure 2-1: Self-assembled nickel nanomatrix formation through femtosecond pulse laser interaction mechanism achieved by programming ultra-short femtosecond laser pulses to ionize nickel

The synthesis of 3D self-assembled nickel oxide nanomatrix by femtosecond pulse laser interaction with nickel target is depicted in schematic Figure 2-1. A computer controlled scanner allowed precise two 
dimensional (2D) movement of ultra-short laser beam over nickel target synthesising 3D self-assembled interwoven nanomatrix composed of nickel and nickel oxide. The nanomatrix generation by this process in the presence of ambient atmosphere is a complex, dynamic and non-linear process, involving a sequence of events: surface energy absorption of laser pulses, plasma plume ignition and ionization, rapid condensation and ejection of ionized species to atmosphere ${ }^{57,58}$. In femtosecond pulse laser interaction process, ultrashort laser pulses with nanosecond pulse to pulse seperation time (ns) and microjoule $(\mu \mathrm{J})$ energy is focused onto the nickel surface, resulting in ionized species formation within the interacting plume which later cools while expanding into ambient atmosphere, forming 3D self-assembled nanomatrix. During this process, only a small focal volume within the plume absorbs the incident energy and transforms the material, minimizing thermal stress and damage $\mathrm{e}^{59,60}$. The control over the expanding plume can be done by adjusting laser pulse energy $(\mu \mathrm{J})$, pulse interaction time (fs) and peak power (MW). Here, the energy deposited by ultrashort laser pulses exceeds inter-atomic binding energy and the atom under excitation becomes free from bonds to interact further with incoming ultra-short laser pulses. This initiates kinectic reaction of ionized species interation with ambient atmosphere in explanding plume. Since laser pulse interaction time is in the orders shorter than material heat diffusion time, it fragments the bulk target material into cystalites and transfigures material from one crystalline structure to another and material recombination ${ }^{61}$. The final ejection of ionised species from the plasma plume outwards forces the superheated ions to cool down as it propels away from interacting surface. They recombine with oxygen present in ambient atmosphere and form 3D self assembled NiO and Ni nanoparticle matrix. The growth rate of each individual nanoparticles within the self-assembled nanomatrix varies with lattice plane orientations and temperature gradient, thereby enabling control over nanoparticle morphology and size. 


\subsubsection{Morphology of nickel oxide nanomatrix :}

The synthesized nanomatrial, as shown in SEM images in Figure 2-2 were a mixture of spherical, cuboidal and hybrid spherodial-cuboidal shaped nanoparticles with varying populations. Most of the bigger particles exhibited spheroidal shape while the cuboidal shaped nanoparticles were significantly smaller in size. Their size distributions, shown in insets of Figure 2-2(A1-A3), are evaluated by estimating the size dimension of all the particles in the each observed image using imageJ software. The average particle size were consistently increasing with the increase of pulse energy from 5 to $20 \mathrm{~nm}$. At lower pulse energy, the nanomatrix is a mixture of nickel and nickel oxide particles. As pulse energy increases, more nickel oxides in the matrix. At the pulse energy of $5 \mathrm{~J} / \mathrm{cm}^{2}$, the entire matrix is nickel oxides.
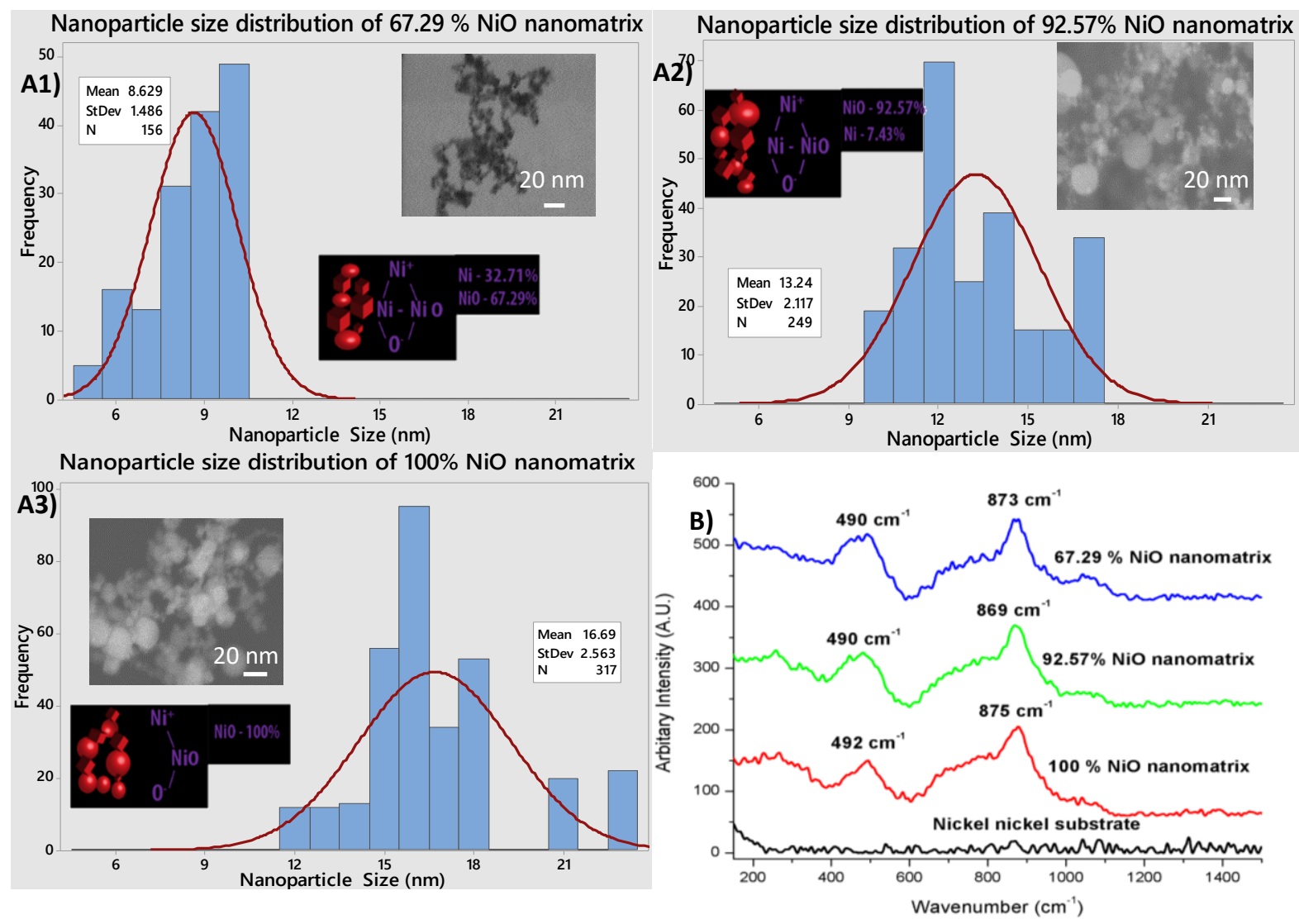

Figure 2-2: Nanoparticle morphology distribution and its Raman activity. Figure 2A(1-3) Summary of nanomatrix morphological distribution. All the images have a uniform scale of $20 \mathrm{~nm}$ B) Raman activity on native and nickel oxide nanomatrix generated at corresponding laser ionization energies 


\subsubsection{Elemental and structural analysis of synthesized nanomatrix:}

The Raman spectrum of crystalline $\mathrm{Ni}-\mathrm{NiO}$ from inset Figure 2-2B shows several bands in the probed region above $300 \mathrm{~cm}^{-1}$. The peak found at $490 \mathrm{~cm}^{-1}$ corresponds with the one-phonon (1P) longitudinal optical (LO) phonon mode of $\mathrm{NiO}$ and the peak at $870 \mathrm{~cm}^{-1}$ can be assigned to two-phonon (2P) 2LO mode based on literature ${ }^{62}$. The first order Raman scattering peak at $490 \mathrm{~cm}^{-1}$ is marginally enhanced and

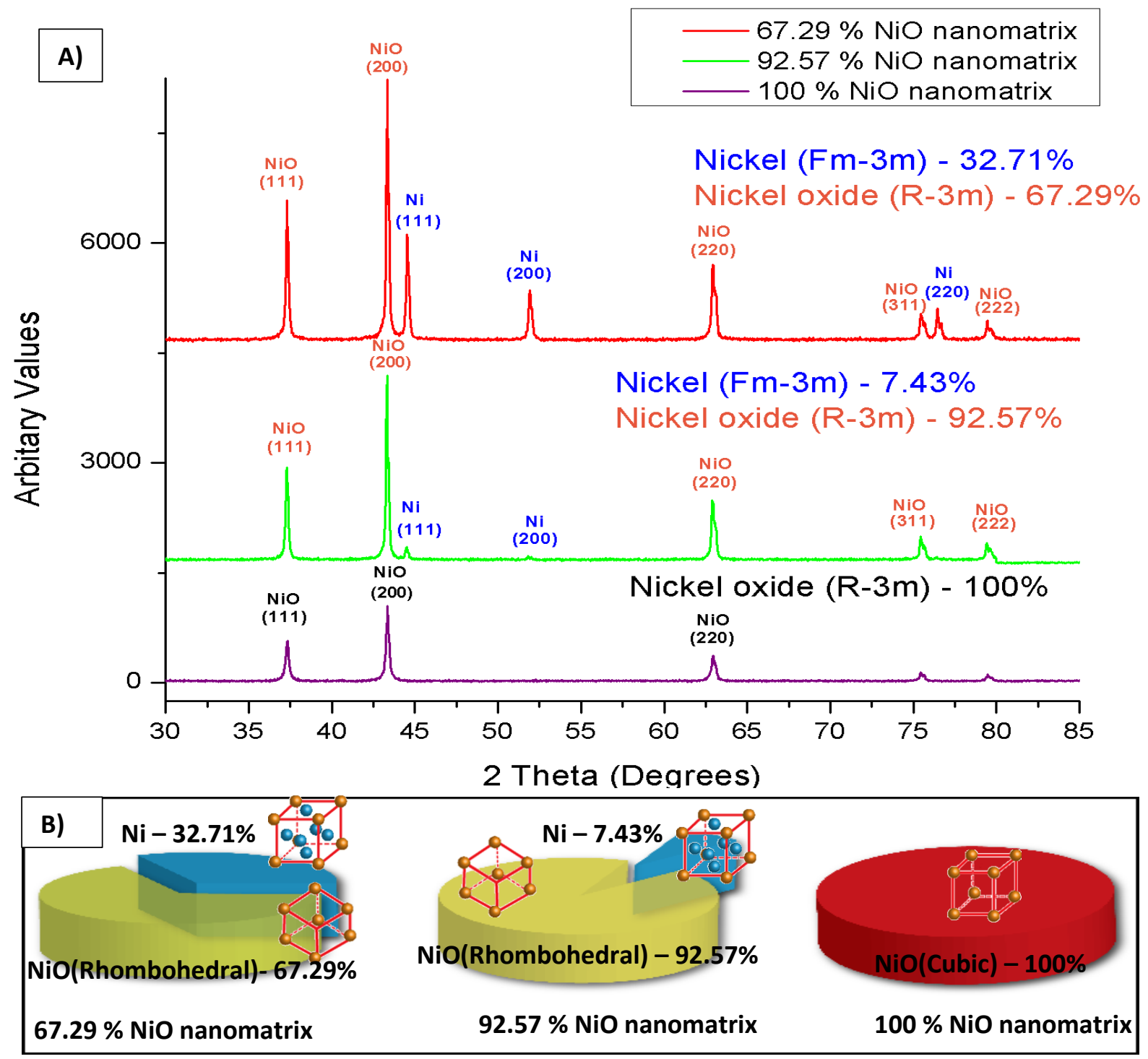

Figure 2-3: X-Ray Diffraction and Reitveld analysis of the synthesized nanomatrix. Figure 3A) XRD spectrum obtained for synthesized nanomatrix B) Elemental Reitveld fitting of observed spectrum is fitted using Pie chart showing the weight percentage of observed phases 
shifted implying high nickel vacancy concentration and the observation of broadend line at $490 \mathrm{~cm}^{-1}$ is associated with the transverse optical mode of NiO. The XRD spectrum analysis on synthesized nickel oxide nanomatrix in inset Figure 2-3A shows a significant line broadening which is often a characteristic phenomenon of nickel/nickel oxide nanoparticles which appear to be in simple cubic and rhombohedral structures with elemental Ni present in significant amount. The reflection peaks for nickel is indexed entirely to face-centered-cubic (fcc) structure \{space group:Fm3m (225)\} of Ni nanocrystal having unit cell dimension: $a=3.5234 \AA$. Three peaks at $2^{\theta}=44.5,51.9,76.5$ corresponds to the diffraction from $\{111,200$, $220\}$ facets, and the peaks at $2^{\theta}=37.28,43.28,62.88,75.28,79.48$ corresponds to the diffraction from $\{111,200,220,311,222\}$ facets. Further analysis did show that the composition remained consistent irrespective of particle morphology and size. Reitveld fitting of the XRD spectrum was performed later to quantitatively approximate the weight percentage of each identified phases constituted in the synthesized nanomatrix inset Figure 3B. The combined presence of nickel and nickel oxide (Rhombhedral) was observed in $67.29 \%$ nanomatrix formed at low laser fluence (i.e.,high laser pulse repetition rate).

\subsubsection{Effect of LDH release of HeLa and NIH3T3 cell in self-assembled nanomatrix:}

HeLa and NIH3T3 cells were seeded on the nanomatrix scaffolds and a native nickel substrate that was used as control sample. The morphology of cells on scaffold was imaged by SEM. The cells were found well spread out from inset Figure (2-6 and A S1). Lactate dehydrogenase (LDH) assay is a tetrameric enzyme that catalyzes the reversible conversion of pyruvate to lactate in the presence of reduced nicotinamide adenine dinucleotide $(\beta-N A D H)$ cofactor. $\mathrm{LDH}$ protein is present within all cells with a resolved crystal structure. The release of this protein is often used as a marker of cell damage in toxicological assays thereby making it an ideal protein for understanding the impact of nanomaterialprotein interaction $^{63}$. For instance, high resolution nuclear magnetic spectroscopy demonstrated that silver nanoparticle decreased GSH (glutathione) and LDH levels, but dramatically increasing amino acid and pyruvate concentration in live cells analysis. This provides the foundation for biochemical mechanism 
for oxidative stress where the depletion of GSH by nanoparticles induce conversion of lactate to pyruvate ${ }^{64}$.

The LDH assay here measures the release of cytosolic enzyme lactate dehydrogenase enzyme when cell membranes are compromised by nickel oxide nanomatrix synthesized. Figure 2-4(A-B) shows the percentage of LDH release from both HeLa cell line and NIH3T3 cell line after incubation of 24 hours and 48 hours, respectively. The control shows elevated LDH release in HeLa when compared to NIH3T3 cell at 24 and 48-hour incubation period. At 100\% NiO nanomatrix condition, LDH release for HeLa, NIH3T3 is elevated from 24-hour and 48-hour indicating that prolonged incubation lead to increase LDH expression. However, when HeLa and fibroblast cell interacts with $67.29 \%$ nickel oxide nanomatrix the LDH release at 24 hours, 48 hours is not elevated when compared to $100 \%$ NiO nanomatrix conditions. This increase in LDH release in both HeLa, NIH3T3 cell line at 100\% NiO nanomatrix condition is because of the unique structural morphology and chemistry of the nanomatrix. 100\% NiO nanomatrix have bigger mean particle size compared to $67.29 \% \mathrm{NiO}$ nanomatrix. Elemental compositions analysis revealed in Reitveld analysis also corroborate that there is difference in their individual composition. One distinct result is that when $\mathrm{NIH} 3 \mathrm{~T} 3$ cell interact with both $100 \% \mathrm{NiO}$ nanomatrix and $67.29 \% \mathrm{NiO}$ nanomatrix condition $\mathrm{LDH}$ release is relatively smaller, which indicates morphology, size and elemental composition didn't play a vital role but rather the nanomatrix were cell specific (self-targeting). Along with the demonstrated cell viability from LDH assays in inset Figure 2-4, cell morphology as observed by SEM inset Figure2-(6,8) and (A-S1).

\subsubsection{HeLa and fibroblast cell response on synthesized nickel oxide nanomatrix platforms:}

With this premise of biocompatibility of our NiO nanomatrix scaffolds with HeLa and fibroblast, we investigated their cell manipulative capacity. NIH3T3 fibroblasts and HeLa cells were cultured both on native nickel and fabricated nickel oxide nanomatrix platforms to investigate their adhesion and 
proliferation response. The SEM results presented in the inset of Figure2-6 compares the HeLa and NIH3T3 fibroblast cell number and morphology response on native and nanomatrix platforms. Both HeLa and LDH release when nickel oxide nanomatrix interacts with HeLa and NIH3T3 cells in 24- hour

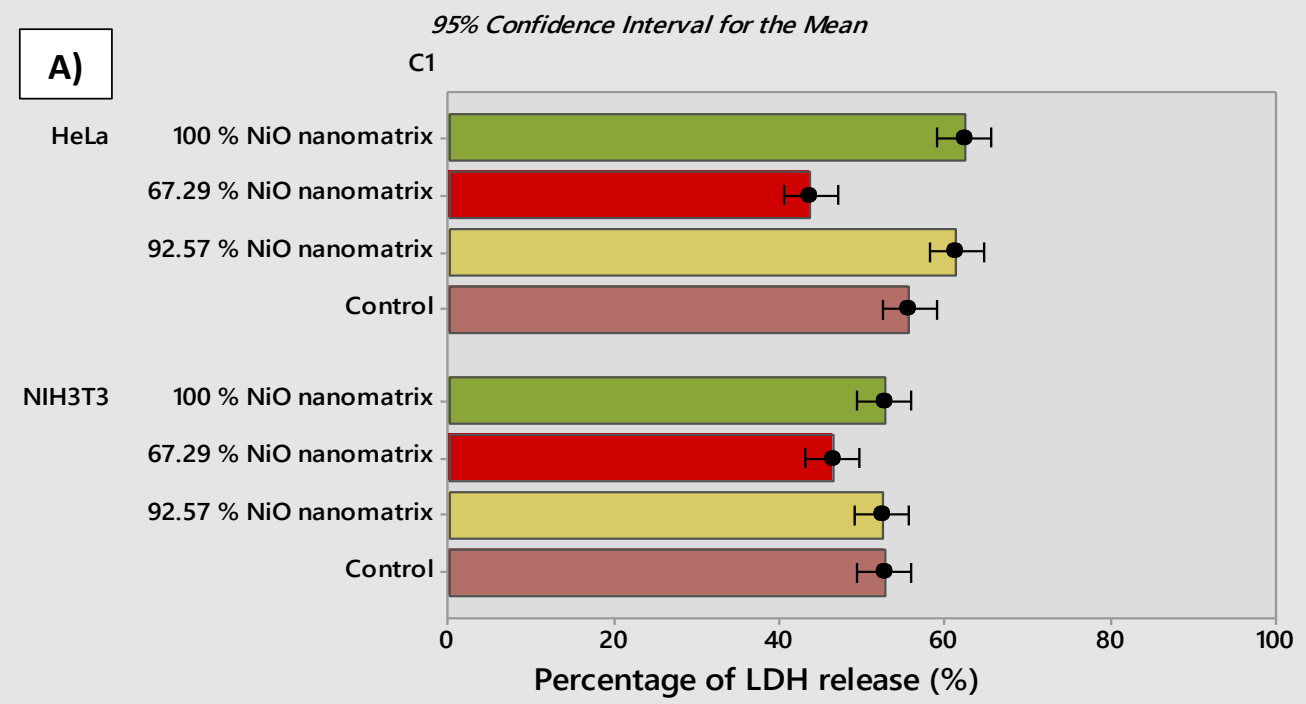

The pooled standard deviation is used to calculate the intervals.

LDH release when nickel oxide nanomatrix interacts with HeLa and NIH3T3 cells in 48-hour

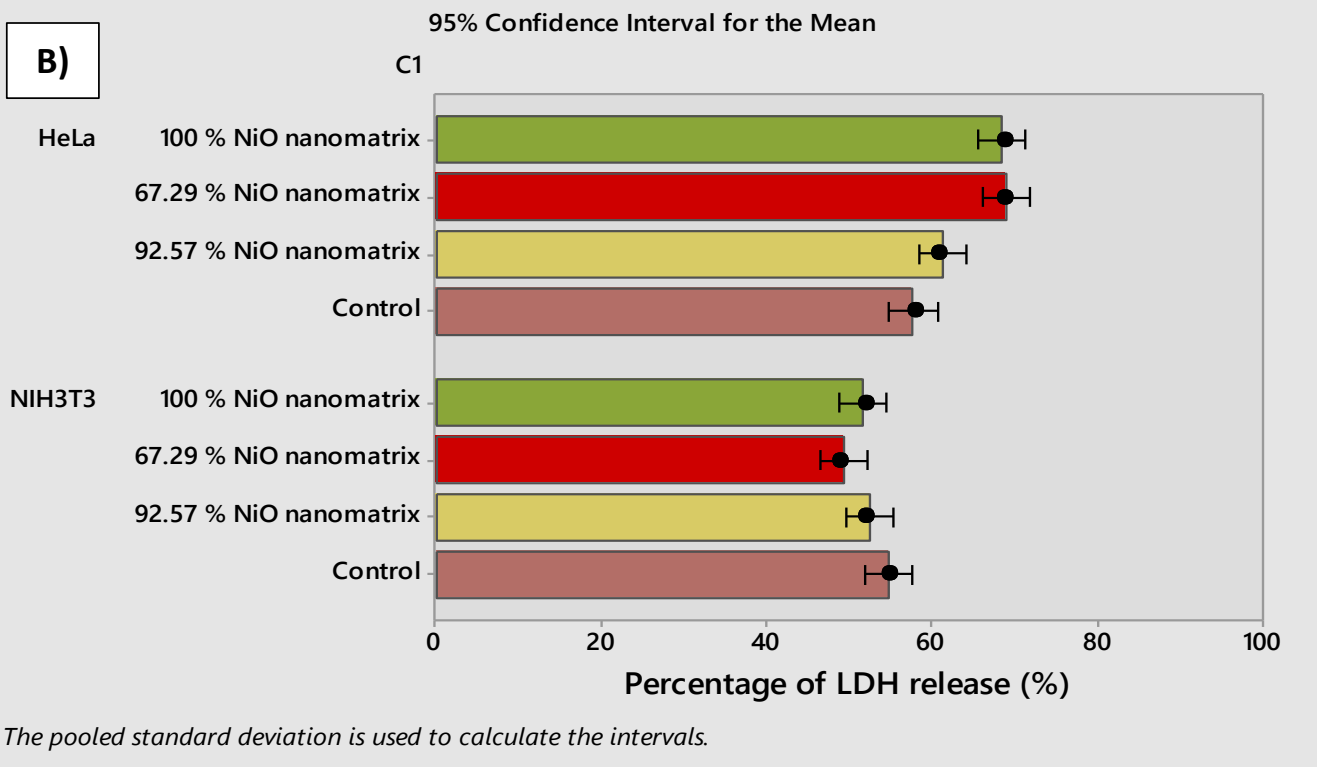

Figure 2-4: Quantifying Lactate dehydrogenase leakage (LDH) by nickel oxide nanomatrix in HeLa and NIH3T3 fibroblast cells. Figure 4 (A) Interval plot determining the $L D H$ release in all NiO nanomatrix for HeLa and NIH3T3 cell in 24 hours, (B) Interval plot determining the LDH release in all NiO nanomatrix for HeLa and NIH3T3 cells in 48 hours

fibroblast cells attached and spread well within 24 hours without any special treatment with ECM molecules prior to cell incubation, indicating the synthesized nanomatrix functioned like ECM. Cells 
incubated on the native flat nickel substrates exhibited normal morphology and almost covered the native substrate, as shown in inset Figure 2-6(I-P).

The fibroblast and HeLa cells proliferated on native nickel were smaller in size and randomly distributed throughout the substrate. However, the number, morphology and organization of HeLa and NIH3T3 cells on nickel oxide nanomatrix were different. First, the size of cells adhered to nanomatrix platform were larger compared to those cells proliferated on native nickel substrates. Secondly, the cells proliferated rather in an organized manner with systematically stretched filopodia and lamellipodia from inset Figure 2-7. The NIH3T3 fibroblast cells present on nanomatrix substrates self-organized closely and almost covered one monolayer thick, making it hard to distinguish one cell from another from inset Figure 2-(6 and 7). A pronounced self-organized cell coverage was observed during the first 24-hour incubation on nanomatrix. This trend continued with prolonged incubation time of 48 -hours on both native nickel and nanomatrix platforms as represented in the inset Figure 2-6. After 48-hours, fibroblast cells on the nanomatrix surface show evidence of elongated cell morphology, suggesting migration. But, the in-vitro response of HeLa cells on the nanomatrix platform revealed remarkable cell governing properties.

At first, we investigated the influence of HeLa cells on nickel oxide nanomatrix which played a key role in modulating cancer cell behavior when compared with fibroblast cells. in the inset Figure $6(\mathrm{~A}-\mathrm{H})$. The nanomatrix platform demonstrated higher degree of Hela cell adhesion compared with fibroblast cells. The statistical results of the same are presented in the inset Figure $2-6(Q, R)$. The total cell count reduced on $100 \% \mathrm{NiO}$ and $67.29 \% \mathrm{NiO}$ nanomatrix platform compared with native nickel control samples after 24 hour incubation. For instance, $67.29 \% \mathrm{NiO}$ nanomatrix presented an $89 \%$ efficiency while $100 \% \mathrm{NiO}$ nanomatrix offered a $65 \%$ efficiency in Hela cell adhesion on nanomatrix surface. $67.29 \% \mathrm{NiO}$ nanomatrix offered a $103 \%$ efficiency of HeLa cell adhesion during initial 24-hour incubation period. 


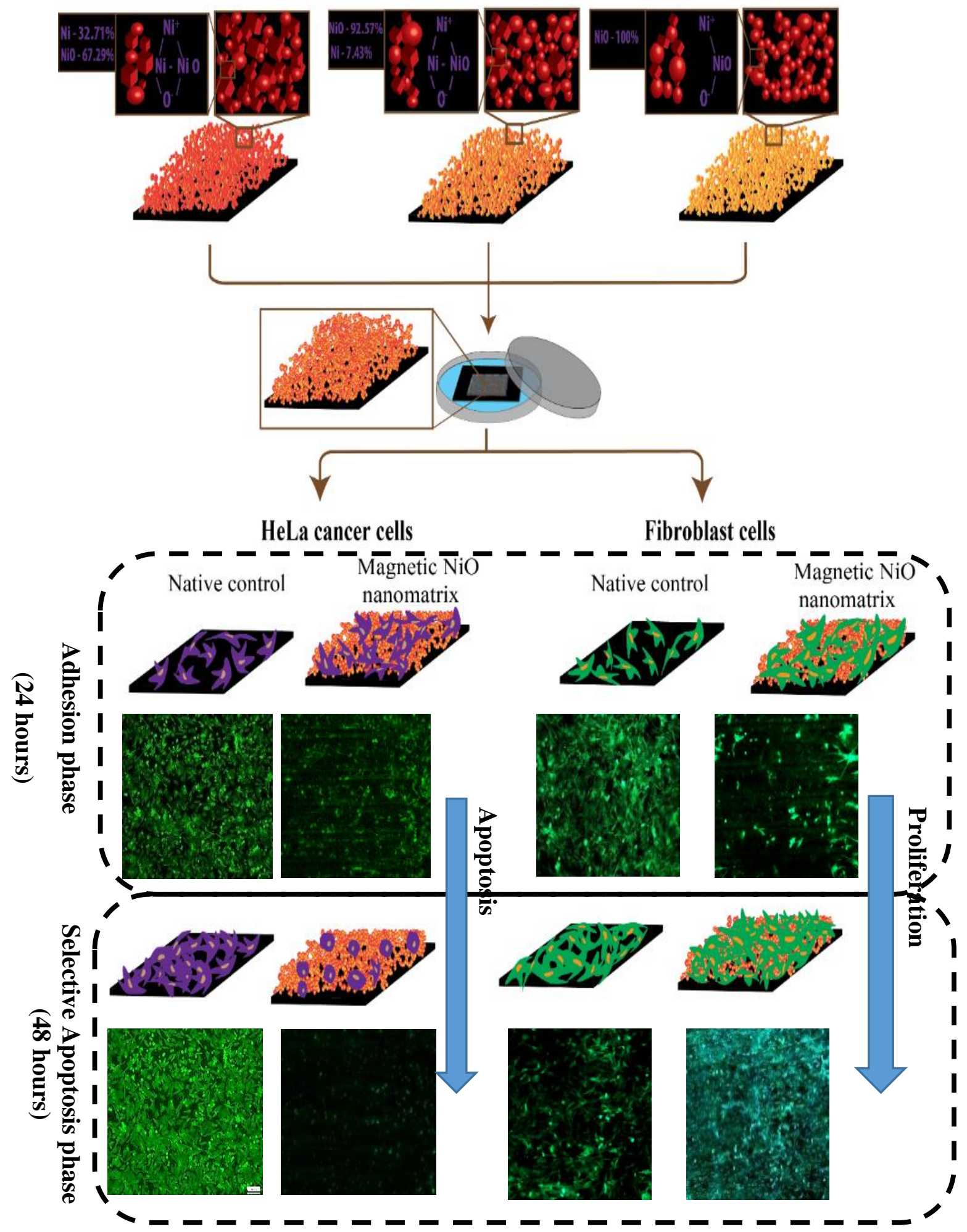

Figure 2-5: Nanomatrix interaction with HeLa and NIH3T3 fibroblast cells. HeLa cells present on nanomatrix show circular morphology (selective apoptosis phase) when comparing with NIH3T3 fibroblast cells 
However, after 48 hours of cell growth, there is straightforward evidence of change in HeLa cell attachment characteristics. There is a complete reversal of cell attachment with respect to nanomatrix morphology wherein, $67.29 \% \mathrm{NiO}$ nanomatrix acted as a magnetic trap by turning off its proliferative behavior and acting as a drug with 48-hour incubation period -94\% cell adhesion efficiency was presented by $67.29 \% \mathrm{NiO}$ nanomatrix. The $92.57 \% \mathrm{NiO}$ nanomatrix which demonstrated an increased HeLa cell adhesion during 24-hour incubation period presented a -9\% efficiency in HeLa cell adhesion during 48hour incubation period from inset Figure 2-(6 $(Q, R)$ and $A-S 1)$. Hence, we can establish a degree of magnetic trapping efficiency in decreasing order based on the observed results with $67.29 \% \mathrm{NiO}, 100 \%$ $\mathrm{NiO}$ and $92.57 \% \mathrm{NiO}$ nanomatrix having high, medium and low degree of attracting behavior. On the other hand, the fibroblast cell adhesivity on nickel oxide nanomatrix doesn't seem to alter even with increased incubation time (48-hour), thus making the attracting behavior explicit to HeLa cells. This infers that both material chemistry and physical architecture of this multitudinal nanomatrix synthesized by varying the ultrashort ionization energy is susceptible to only HeLa cells. These results thus substantiate the theory that cells sense subtle change in the microenvironment and react via transmitting extracellular signals to the nucleus ${ }^{65,66}$.

It is observed from the presented results inset Figure 2-(6 and A-S1) that NIH3T3 cells is less susceptible to multitudinal architecture of $\mathrm{NiO}$ nanomatrix when compared to HeLa cell. Earlier studies on extracellular matrix (ECM) have demonstrated its vital role in regulating cell adhesion, sensing and signaling. Emerging works in 3D scaffold have pointed out the need for incorporating ECM proteins and specific biomolecules to alter cell morphologies, adhesion, proliferation and migration ${ }^{67,68}$. The 3D porous structures have shown promise when compared with its one and two-dimensional counterparts in cellular 


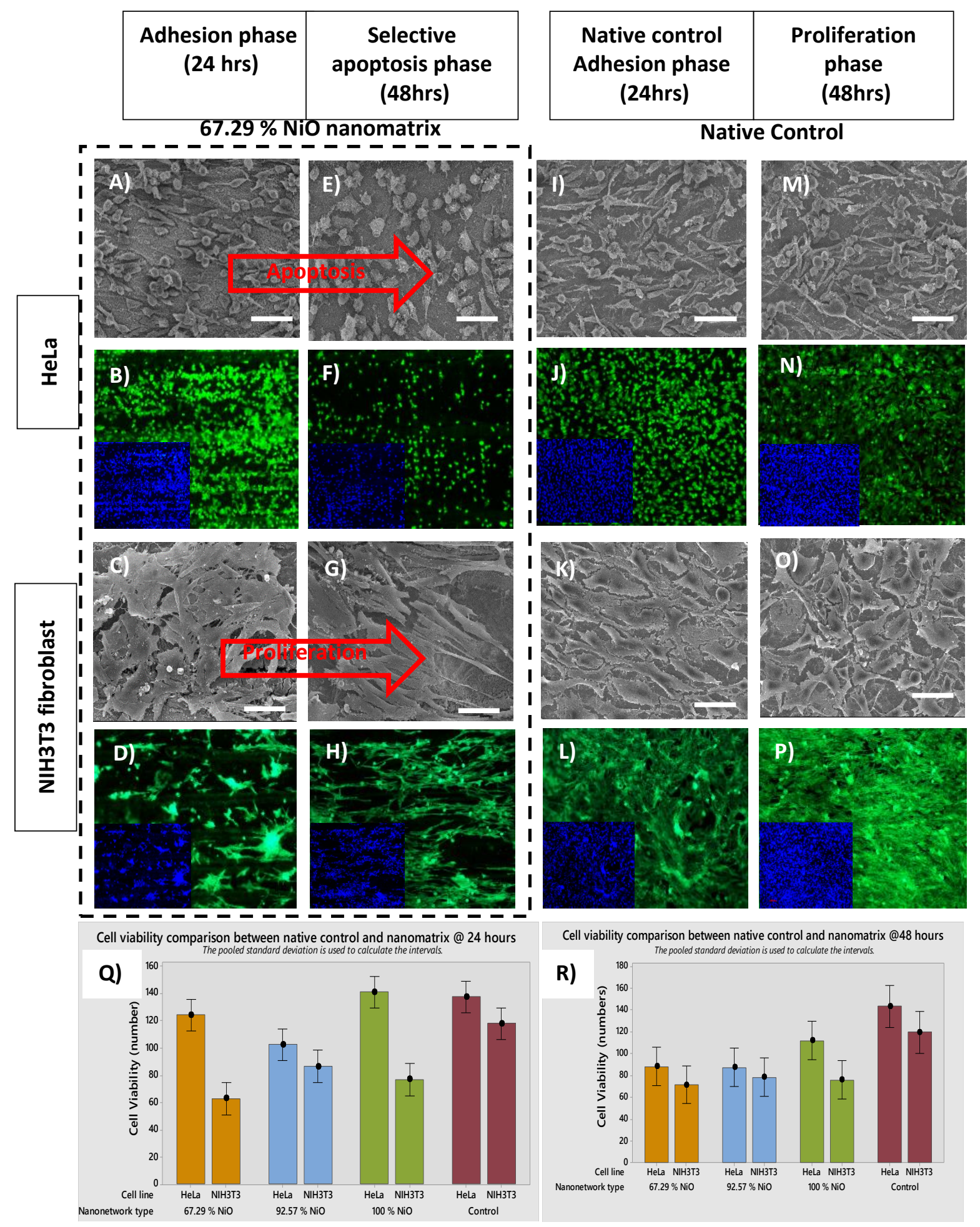

Figure 2-6: Quantifying HeLa and NIH3T3 Fibroblast cell proliferation on synthesized nanomatrix. A comparison between HeLa and NIH3T3 fibroblast cell interaction on synthesized nanomatrix and unprocessed native nickel control surface for 24 hours and 48 hours. (A-H) fabricated platforms and (IP) unprocessed native nickel for 24 to 48-hour incubation. $Q$ and R) Graphical representation of HeLa and NIH3T3 fibroblast cells on nanomatrix interaction after 24 and 48 hours as compared to control native nickel substrate All the fluroscence images have a uniform scale of $10 \mu \mathrm{m}$ 
adhesion and migration due to its heterogeneous nature ${ }^{66}$. Unlike conventional anti-cancer agents that attack cancer cells right from the beginning, the nickel oxide nanomatrix synthesized here is selffunctionalized. It presented favorable cues to HeLa cell initially (24 hours), thereby promoting adhesion and proliferation. But, then apoptosis is then induced after prolonged incubation time of 48-hour. Moreover, the apoptosis is induced solely by the nanomatrix, making it a medicine rather than a mere carrier or agent as compared with other cancer therapy strategies ${ }^{69-71}$. A schematic illustration of this trend is presented in inset Figure 2-5.

\subsubsection{Role of nanomatrix morphology and chemistry in cell-nanomaterial interaction:}

The synthesized nickel oxide nanomatrix resembles a 3D ECM platform that is randomly interlinked with varying porosity. Figure 2-7 ((A, B) and A-S1) illustrates the impact of nanoparticle morphology and chemistry present within the nanomatrix on interacting cells. A varying degree of cell morphological and population (count) is observed for both fibroblast and HeLa cells irrespective of type of nanomatrix $67.29 \% \mathrm{NiO}, 92 \% \mathrm{NiO}$ and $100 \% \mathrm{NiO}$. It is also seen that nanomatrix severely reduced the population of HeLa cells by altering the morphology cells with increased incubation time (48-hour).

The nanoparticles present at the interface of the nanomatrix and substrate are strongly adhered to each other and are tightly bonded to the substrate whereas, the nanoparticles on the top of nanomatrix surface are all loosely packed. Therefore, their adherence to the cells are relatively weak. This transformation presented by nanoparticles in the nanomatrix plays a significant role in its movement within the interacting medium which the sub-layer nanomatrix lack presented in inset Figure 2-8. The primitive role played by the nanomatrix is in controlling cell populations by encapsulation and triggering endocytic reaction ${ }^{72,73}$. The individual role played by nanomatrix morphology on cells is highlighted in inset Figure 2-7. The HeLa cells interacting with both $67.29 \% \mathrm{NiO}$ and $100 \% \mathrm{NiO}$ nanomatrix presented elongated structure (lamellipodia) with sporadic filopodial sensing during 24-hour incubation from inset Figure 2-7A. 


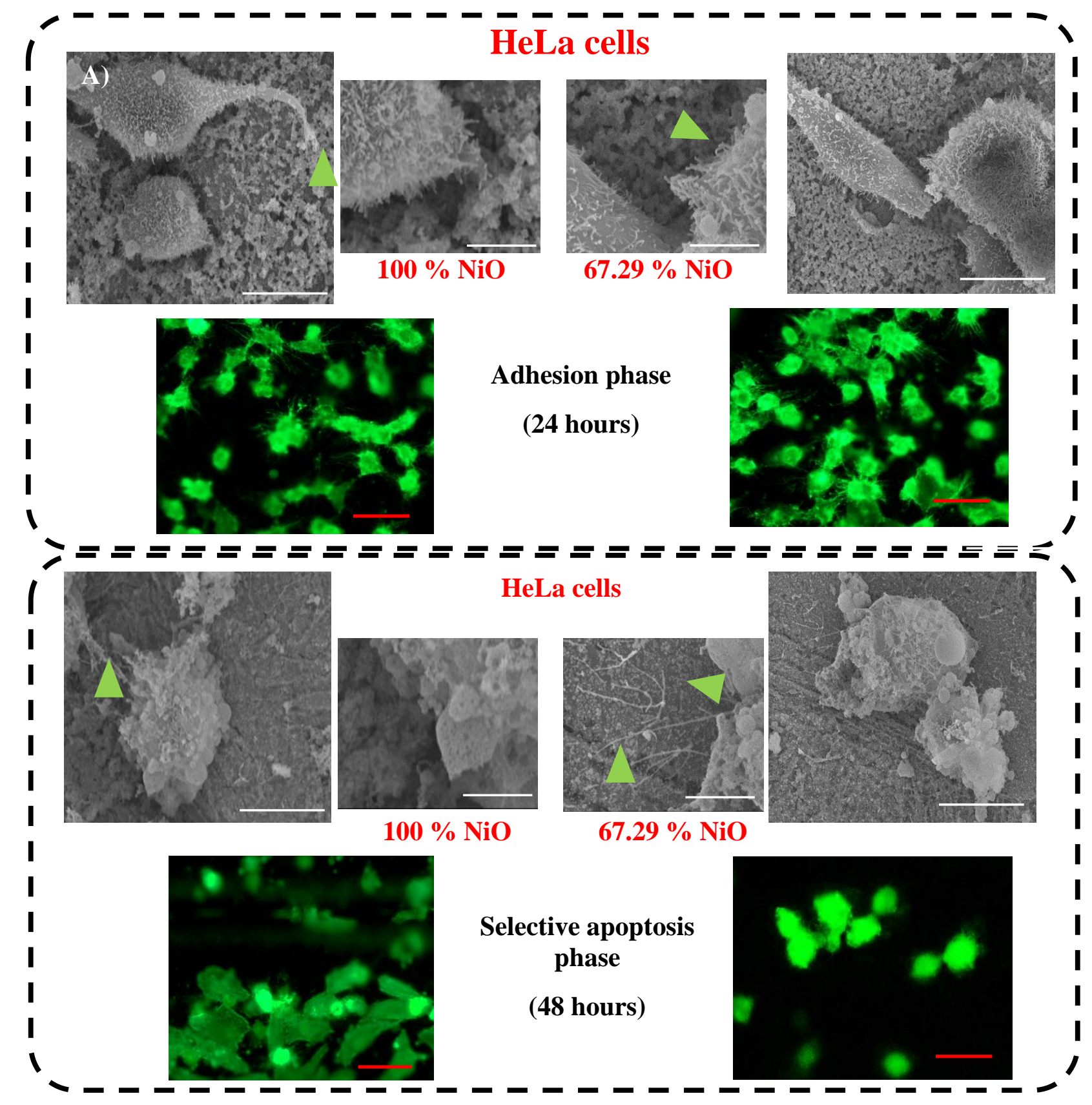




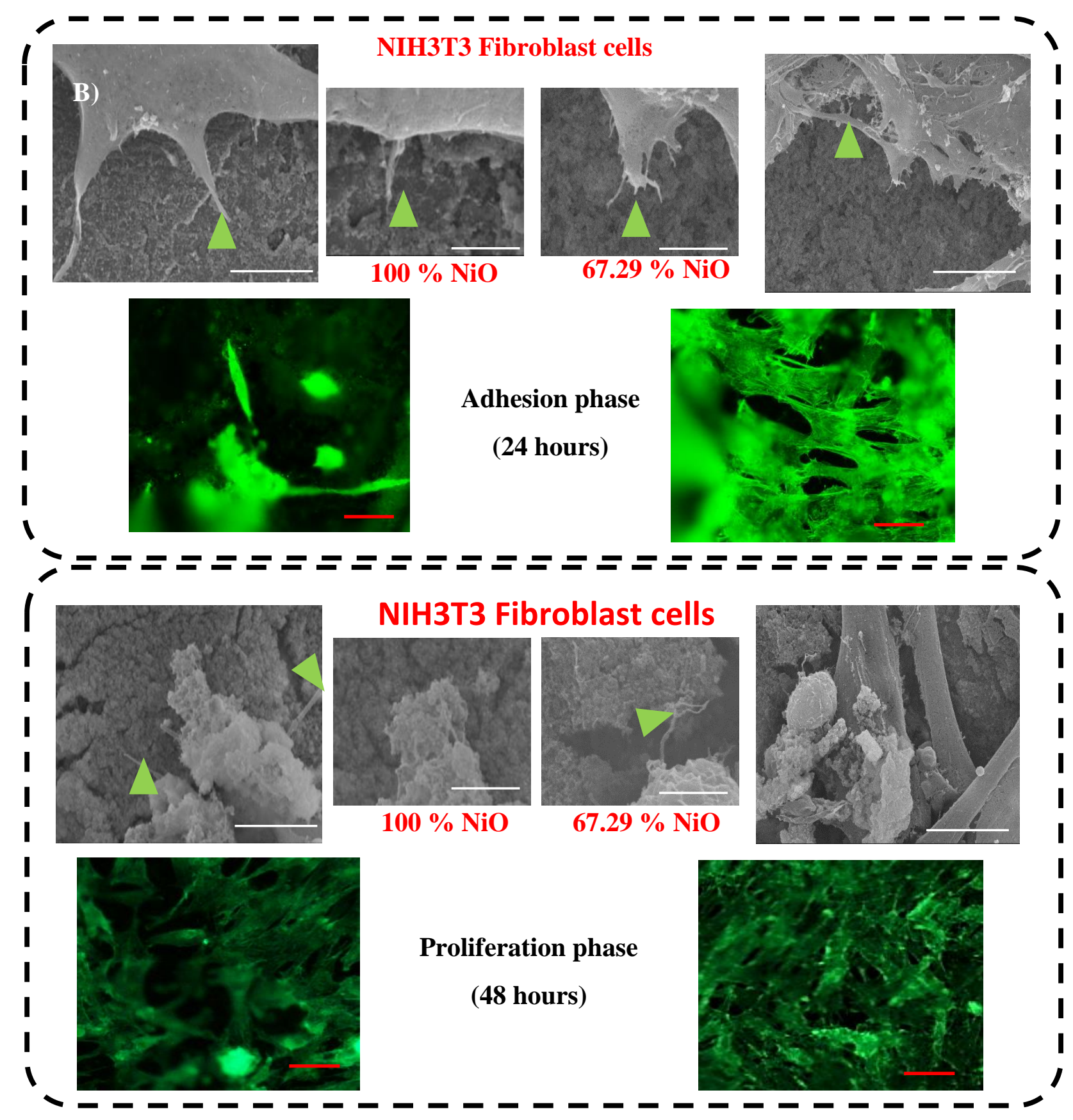

Figure 2- 7: Influence of different nickel nanomatrix on adhesion (24 hours) and proliferation phase (48 hours). A) Comparison between the $67.29 \%$ NiO and $100 \%$ NiO nanomatrix platforms over HeLa cell adhesion. B) Comparison between the $67.29 \%$ NiO and $100 \%$ NiO nanomatrix platforms over NIH3T3 fibroblast adhesion. A uniform scale bar of 10 and $3 \mu \mathrm{m}$ was followed. The green indicators are filopodial presence and their interaction on nanomatrix.

But, the increase in incubation time to 48 hours led to altered cell morphological behaviour. The $100 \% \mathrm{NiO}$ nanomatrix although induced a contractile behaviour, evidence of filopodial activity was still present. Nevertheless, the $67.29 \% \mathrm{NiO}$ nanomatrix presented no such response to HeLa cells with predominant 
cells in round/circular morphology from inset Figure 2-7A. However, fibroblast cells presented in inset Figure 2-7B did not exhibit any such behaviour to nanomatrix platform rather showed augmented filopodial and lamellipodial activity irrespective of incubation period.

\subsubsection{Effect of different shaped nanomatrix on cell morphological characteristics:}

In addition to total cell population, a comparative quantitative analysis was evaluated on fibroblast and HeLa cell morphology interacting with nanomatrix surface. Interacting cell morphology is often a good indicator of cell response to nanomatrix. Here, we observe three kinds of cellular morphologies from the inset Figure (2-8) - round, elongated and amoeba SEM observations from inset Figure 2-(6, 7 and A-S1) have found that nanomatrix induced more morphological alterations than native control surface where the observed cells were predominantly round and elongated. The rounded morphology of cells without filopodial attachments indicate that they don't prefer the site for adhesion and subsequent proliferation and then the nanomatrix trap the cells by encapsulation. Elongated cellular morphology is indicative of cell proliferation and migration on interacting surface. Premanth et al., proposed that cell adhesion to silicon nanostructures are highly material dependent with fibroblast cells being less sensitive to surface properties of silicon nanostructures when compared with HeLa cells ${ }^{74}$.There are some reports which have also shown that nanostructures encourage cell adhesion, proliferation and migration. For instance, Tavangar et al., showed titanium nanostructures promoted fibroblast and osteoblast cell adhesion ${ }^{75}$. Also, there are studies which indicate nanostructures hamper cell adhesion and migration. However, Chinnakkannu Vijayakumar et al., showed titanium nanostructures aided in distorting both fibroblast and HeLa cell adhesion through nanostructures material chemistry alteration by ultra-short laser ${ }^{76}$. The nickel oxide nanomatrix synthesized here has bi-functional property where it encouraged HeLa cell adhesion during initial incubation (24-hour) acting as ECM and induced apoptosis to HeLa cell growth after 48 hours acting as a drug thereby self-targeting. This may be attributed to the difference in cell shape and population observed from inset Figure2-(6 and 7). Only a few number on HeLa cells survived after 48 
hours.

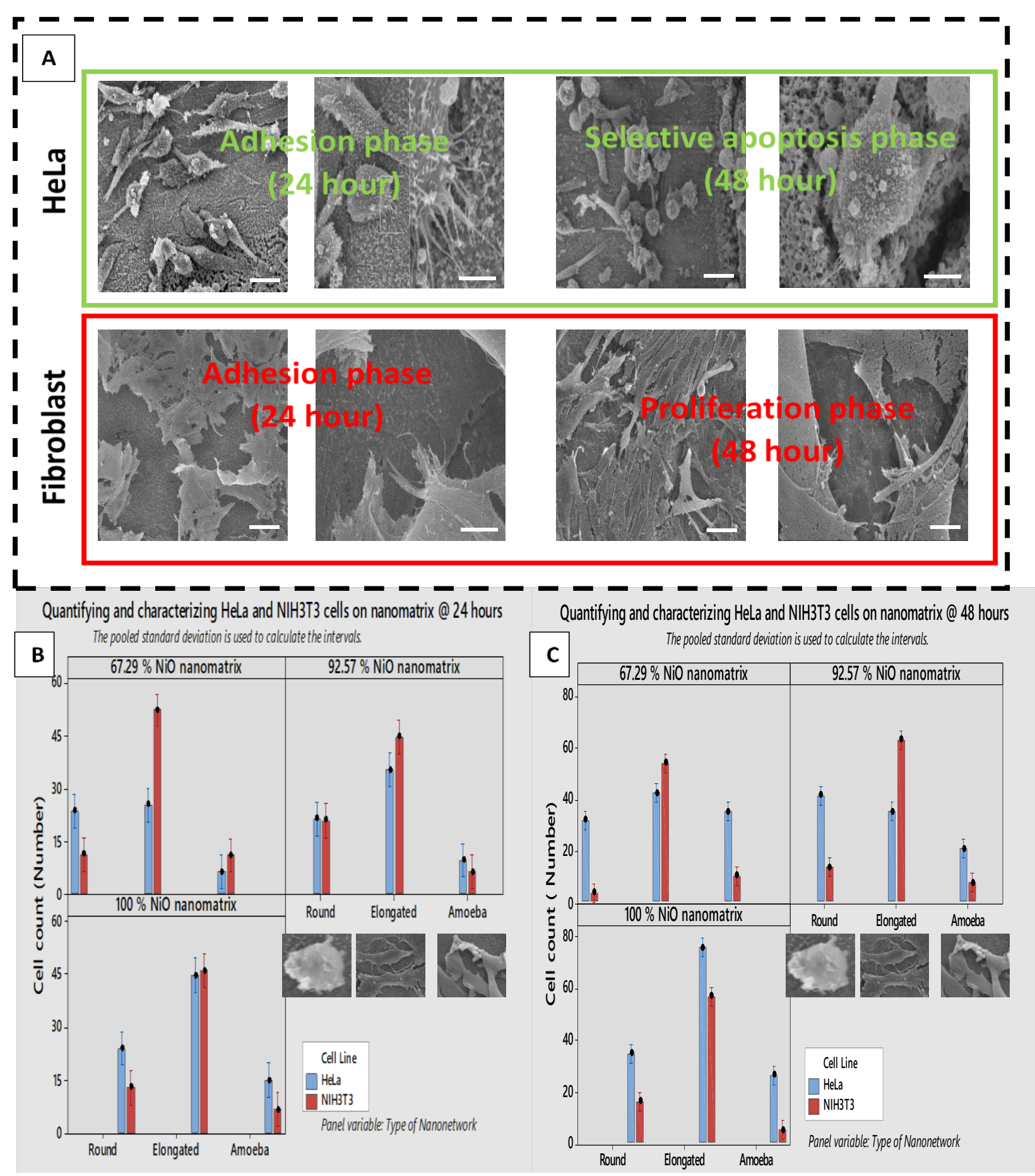

Figure 2-8: Quantifying and characterizing HeLa and NIH3T3 fibroblast cells based on shape. A)

Representative HeLa and Fibroblast exhibiting morphological variance at 24 and 48-hours on nanomatrix B and C) HeLa and fibroblast cell exhibiting different morphologies on nanomatrix at 24 and 


\section{8 hours (results are represented as mean $\pm S D ; n=3$; counted in triplicate in individual microscope}

fields). All the SEM images have a uniform scale of $10 \mu \mathrm{m}$

The trapped cells shrunk into small round shape and were missing filopodia and lamellipodial extensions, as shown in inset Figure 2-(6, 7, 8 and A- S1). The increased incubation time from 24 to 48 hours triggered atypical behavior response in HeLa cells which can be attributed to both nanomatrix material chemistry (oxide and phases) and material morphology. On the other hand, it is also observed that HeLa cells adhered on native surface developed into large polygonal (amoeba) shape with numerous filopodia extensions. The fibroblast cells cultured both on native nickel substrate and synthesized nickel oxide nanomatrix platforms are to study adhesion and proliferation of normal cell line as a control for the investigation. The inset Figure 2-(6, 7 and 8$)$ compares the cell number/count and morphology of fibroblasts attached on native nickel and nickel oxide nanomatrix. The SEM and fluorescent images shown in the inset Figure 2-(6,7 and A-S1) suggest that fibroblast cells attached and spread on both substrates within 24 hours. However, the number, organization and morphology of cells attached to nickel oxide nanomatrix were different than those attached to native nickel. The fibroblasts grown on native nickel were well spread(amoeba) with even size distribution throughout the substrate. Whereas, cells attached to nickel oxide nanomatrix platform adhered in clusters with their filopodia and lamellipodia systematically stretched along the nanomatrix areas. Their size was also relatively large compared to those cells grown on native nickel substrate and hence, the number of cells also present on nickel oxide nanomatrix was less. However, there remains a question as to why number of cells were observed on nanomatrix surface when compared to the native nickel surface. The size of the fibroblast cells was observed to increase on both native nickel and nickel oxide nanomatrix platforms after 48 hours. Confluent tissue like layers of cells were evident on nickel oxide nanomatrix seen from inset Figure 2-(6 and 7). 


\subsubsection{Physiological study of interacting cells with synthesized nanomatrix:}

Apoptotic cell death is often associated with a set of distinct biochemical and physical changes involving cell cytoplasm, nucleus and plasma membrane ${ }^{15}$. In early stage of apoptosis, cells round up, losing contact with surrounding cells and shrink. The filopodia of interacting HeLa cells observed via SEM from inset Figure 2-(6 and 7A) was employed to study the adhesion and proliferation properties of cells on adhesive nanomatrix when compared with native nickel substrate. The filopodial development on native nickel is random. In contrast, cells interacting on nanomatrix showed a drastic change in the number of filopodia during the first 24-hour incubation. The cells that proliferated had on average lesser filopodia with altered morphology when compared with native nickel substrate. It is known that cells need to spread to stratify various phases of cell growth. Thus, filopodia that adhere to substrates typically originated from random locations on the cell body and at certain distances above the surface. These filopodia thus contact the underlying substrate under a broad range of angles from inset Figure 2-7A. It could also be seen that not only were filopodia interacting with the nanomatrix, but so were the retraction fibers of the cell from inset Figure 2-7A. The presence of filopodia and retraction fibers seen at end points demonstrate the fact of occurrence of high incidence of cellular and nanomatrix interaction having taken place.

\subsubsection{Cytoskeletal alterations by nickel oxide nanomatrix:}

Cell cytoskeleton primarily maintains cell shape and plays a significant role in intracellular transport, cell motility, division and force generation ${ }^{77}$. When nanomaterials interact with cell cytoskeleton they directly or indirectly alter their behaviour due to endocytic cycle. The loss of this cytoskeletal function lead to decreased cell motility, division and proliferation as observed in HeLa cells from fluorescent images in inset Figure 2-(6 and 7). The $100 \% \mathrm{NiO}$ nanomatrix show bright focal adhesion points and actin stress fibers. Since, stress fibers communicate surface properties of surroundings to the cell, the stretched and flattened cytoskeleton here show proliferation of cell. In addition, the $67.29 \% \mathrm{NiO}$ nanomatrix present 
excess stress fibers showing their cytoplasmic response to interacting nanomatrix present in inset Figure 2-(6 and 7).

However, with increased incubation time of 48 hours Hela cells indicate the lack of stress fibers on the cells demonstrating lack of cell adhesion on the $67.29 \% \mathrm{NiO}$ nanomatrix. Therefore, it could be considered as a pre-indicator of cell death. In-addition, it is observed that cell nucleus does not align to the center of cell but, seem of arranged in random fashion. The shape and size of the nucleus depends on type of nanomatrix the cells interact with. The cell nucleus that are in contact with nanomatrix are elliptical owing to cell cytoskeletal forces. It is clear a difference in nuclei is observed across the nanomatrix interacting with the cells. Well-rounded nucleus in cells proliferating on native nickel substrate show the marked difference in cell sensitivity to nano cues from synthesized nanomatrix. The significant reduction in cell nucleus observed on nanomatrix when compared to nuclei on native nickel surface indicate that a reduction in nucleus size may induce physicochemical reaction leading to apoptosis. Altered nucleus size is indicative of chromatin re-arrangement and gene expression as explained by Webster et $\mathrm{al}^{78}$. The altered gene expression observed here might be a potential factor for the substantial number of rounded cells on nickel oxide nanomatrix. The detection of trace existence of nickel and nickel oxide nanoparticles from nanomatrix in cellular membrane was identified using EDX. The elemental mapping presented in inset Appendix Figure A $(S 2, S 3)$ elucidate the delayed the apoptotic behaviour exhibited by nanomatrix on HeLa cells when compared with fibroblast cells. It is evident from elemental mapping information that both HeLa and fibroblast cells had adhered and proliferated on the nanomatrix based on their spreading area. The individual elemental mapping information in inset Appendix Figure $A(S 3(A, B)$ and S2) for carbon show HeLa and fibroblast cell spreading and proliferation. The internalization of interacting $67.29 \% \mathrm{NiO}$ nanomatrix and $100 \% \mathrm{NiO}$ nanomatrix is also evident in both fibroblast and HeLa cells. Elemental nickel (highlighted in red) is safely internalized by HeLa cells in adhesion phase ( 24 hours) and then observed to be present only outside the HeLa cells in selective apoptosis phase (48 hours) from inset image Appendix 
Figure A-S3A. This supported by nanoparticle illumination from corresponding fluorescence images. On the other hand, in NIH3T3 fibroblast cells the elemental nickel nanoparticles are present surrounding the cells in adhesion phase ( 24 hours) and then present both inside and outside the cell during proliferation phase (48 hours) from inset Appendix A-S3B. In addition, the presence of elemental oxygen surrounding the cells in inset Appendix Figure A (S3(A, B) and S2) did not change between HeLa and fibroblast cells for 24 hours suggesting there is no adverse change in cell proliferation. However, at increased incubation time (48 hours) we see an increased oxygen presence in HeLa cells when comparing with fibroblast cells. This observed change in oxygen utilization by cells is indicative of apoptotic response from cells.

\subsection{Conclusion:}

An experimental study was made by applying magnetic NiO nanomatrix for cellular interactions with immortal HeLa cancer and fibroblast cells to determine its use as a nanostructure platform to serve as a drug-free nanomedicine for cancer therapy. The cellular response with respect to adhesion, proliferation and cytotoxicity of interacting cell lines on three $\mathrm{NiO}$ nanomatrix were studied. We observed a superior anti-tumor response by $\mathrm{NiO}$ nanomatrix wherein the nanomatrix imitated ECM like behavior on cancer cells for 24 hours and followed by a selective apoptosis of HeLa attachment efficiency shifting from 103\% to $-94 \%$ after 48 hours period. Nevertheless, the fibroblast cells proliferated upon the nanomatrix inducing negligible cytotoxicity mimicking tumor microenvironment irrespective of the incubation time period. This selective apoptosis of HeLa cancer to have drug like property is brought about by this specific NiO nanomatrix while acknowledging the fact that the self-targeting phenomenon resulted from the 3D structure formed. Our results also showed that nanomatrix quantitatively altered HeLa cell behavior wherein evidence of increased endocytosis was also demonstrated. Moreover, additional new insight reveals as to how fibroblast cells behaved differently when compared to those of HeLa cells on nickel oxide nanomatrix platforms. This 3D drug-free nanomatrix structure synthesized using femtosecond laser 
pulse interaction mechanism by application of femtosecond laser pulses facilitated the synthesis of $\mathrm{NiO}$ nanomatrix by appropriate variations made in nanomaterial chemistry. There has been no previous reporting on synthesizing 3D drug free self-targeting nanomatrix with programmable material morphology and chemistry for cancer treatment. We hope our study can offer better insight to generate drug-free magnetic nanomatrix using nickel in future as a cancer therapeutic application in future. 


\section{CHAPTER 3}

\section{D QUANTUM THERANOSOMES: A NEW DIRECTION FOR LABEL-FREE THERANOSTICS}

Flourishing development of quantum scale materials offer great potential in the field of cancer theranostics. Presently, quantum materials are severely limited due to OD \& 1D material which lacks biocompatibility resulting in coated materials with labelled tags for fluorescence excitation. In-addition, application of magnetic quantum materials has never been reported till date for cancer theranostics. In this current research study, we introduce the concept of applying nickel based magnetic 3D quantum theranosomes for label free broadband fluorescence enhancement and cancer therapy. To begin with, we now present two (primary and secondary) distinct quantum theranosomes for cancer detection and differentiating (HeLa \& MDAMB-231) from mammalian fibroblast cells. The primary theranosomes exhibit Metal Enhanced Fluorescence (MEF) property through localized surface plasmon resonance to act as cancer detectors whereas the secondary theranosomes act as cancer differentiator through fluorescence quenching of HeLa cancer cells. Apart from the above, the synthesized magnetic quantum theranosomes introduced therapeutic functionality wherein, the theranosomes mimicked a tumor microenvironment by selectively accelerating the proliferation of mammalian fibroblasts cells while at the same time inducing cancer therapy. These quantum theranosomes were synthesized using a femtosecond pulse laser and thereafter was self-assembled by top-down approach to attain the desired 3D quantum structure morphology. The 3D arrangement combined with the physicochemical properties of quantum theranosomes mimicked a tumor microenvironment. Furthermore, we anticipate that our current findings recorded can further shed light upon these unique magnetic quantum theranosomes as a potential contender towards opening an entirely new direction in the field of cancer theranostics. 


\subsection{INTRODUCTION:}

Theranostics is an integration of a dual function system that offers both cancer diagnostic imaging and concurrent therapy merged into a single operating platform. Cancer theranostics have now gained momentous importance in the recent years by offering targeted delivery through conjugates facilitating both chemotherapy and bioimaging through novel strategies in oncological research ${ }^{79}$. Cancer theranostics in recent times require non-invasive imaging techniques to track the progress of the therapeutic cells. Currently optical imaging (fluorescence, bioluminescence and photo acoustics) is one such technique offering a remarkably high sensitivity amongst a variety of cell tracking techniques. It is noteworthy to mention at this stage that all the above-mentioned techniques require the use of multiple specific labels or tags to accomplish the desired imaging using nanoscale materials ${ }^{80}$. Nevertheless, there are no reported works on label-free fluorescence bioimaging adopted or already implemented for cancer theranostics.

Primarily, to achieve label-free fluorescence bioimaging, one worthwhile approach is moving towards quantum scale materials thereby opening a new direction in cancer theranostics. Quantum dots (QDs) particles with fluorescence excitation are a highly desired feature for cancer theranostics. These quantum scale (QS) materials have been functionalized externally to be used as fluorescence probes in biolabeling and bioimaging applications due to its unique high quantum yield, molar excitations co-efficient level, optical and electronic, properties ${ }^{81-83}$. These unique properties exhibited by the quantum scale materials are due to the quantum confinement effect observed in metals and semiconductor materials considered that are of 2-8 $\mathrm{nm}$ in size. These materials in turn share its dimensional similarities in terms of proteins, and nucleic acids in the desired new biological environment ${ }^{84,85}$. Nevertheless, conventional fluorescence cell imaging which utilizes fluorophores suffer major drawbacks namely due to non-specific fluorophore accumulation, short lifetime (ns to $\mu \mathrm{s}$ ) with progressive photobleaching process and possess very limited contrast features that hinder their use in theranostics applications ${ }^{86}$. Hence, there is now an inherent need 
for an apt diagnostic method to overcome all the above said limitations offered by these fluorescent molecules for cancer theranostics.

Albeit, all quantum scale materials in general exhibit many several attractive characteristic properties such as size-dependent tunable fluorescence, high brightness, single wavelength excitation and large surface to volume ratios. In addition to the above, they also resist photobleaching and chemical degradation when compared with the available conventional dye ${ }^{87}$. But, the use of these QDs for theranostics were severely restricted due to the innate cytotoxicity, particle size dependency for excitation, single wavelength excitation and the need for bioconjugation labels for in-vitro application to induce fluorescence excitation $^{16,17}$. Thus, there is an overwhelming need for quantum scale materials which addresses all the aforementioned drawbacks of QDs to achieve a potential label-free cancer theranostics agent.

Some successful attempts were reported addressing a part of the above-mentioned drawbacks using coated QDs and noble-metal doped QDs for fluorescence bioimaging applications ${ }^{88-90}$. These are often nanoscale polymeric coating or tripeptide coating over QDs to help in improving biocompatibility thereby overcoming toxicity. Similarly, QDs are limited to specific wavelength excitation due to particle sizes and thus different particle sizes synthesized using a variety of synthesis techniques are combined to achieve the desired broadband fluorescence excitation. Furthermore, these QDs are limited to OD and 1D materials which do not possess the physiochemical characteristics to mimic a tumor microenvironment to be effectively deployed for cancer theranostics application thereby severely limiting its use. Therefore, to the best of our knowledge, there exists no reported works on the use of 3D label-free magnetic quantum probes with broadband fluorescence excitation for cancer theranostics.

Moreover, to satisfy the use of cancer fluorescence bioimaging probes for cancer theranostics application; there is a need for a viable transport mechanism. This can essentially be fulfilled using magnetic materials to achieve the desired site-specific reaction at the targeted site through a variety of external targeting approaches, for instance Magnetic Resonance Imaging (MRI) and thermal imaging ${ }^{91,92}$. Both these 
methods however are not only time consuming but the Enhanced Permeability and Retention (EPR) effect has severely limited its use in fluorescence bioimaging application ${ }^{93}$. Some successful attempts were demonstrated along the same lines using magnetic QDs as a viable alternative to fluorescent dyes for bioimaging applications. These were achieved using magnetic materials like Nickel, Gadolinium and Iron by a variety of techniques adopted such as magnetic doping, functionalized ligands and antibody coatings for labelled multimodal imaging ${ }^{94-96}$. While the magnetic materials addressed the targeting aspect, the issue of biocompatibility of these materials has also not been fully evaluated for bioimaging applications. In-addition, to be successfully deployed for cancer theranostics, these magnetic probes must also necessarily induce cancer therapy. So far, magnetic materials are widely used as only as drug delivery agents rather than to induce cancer therapy. Nevertheless, till this date there is no reported work published regarding the use of magnetic quantum materials for label-free cancer theranostics.

In this research study, we report the development of nickel based magnetic quantum theranosomes for cancer theranostics. Two (primary and secondary) theranosomes were synthesized in this research to achieve a label-free broadband fluorescence excitation and for cancer therapy. The primary (quantum-Ni) theranosomes acted as a cancer detector by exhibiting fluorescence intensity only upon interacting cancer (MDAMB-231 and HeLa) cells when compared with NIH3T3 fibroblast cells. This fluorescence enhancement is achieved through localized surface plasmon resonance giving rise to metal enhanced fluorescence excitation. The secondary (quantum-NiO) theranosomes acted as cancer differentiator distinguishing between the interacting cancer cells by selectively demonstrating increased fluorescence intensity in MDAMB-231 cancer cells when compared with HeLa cancer cells. The secondary theranosomes chiefly composing of metal oxide, induced energy dependent fluorescence enhancement through theranosome localization thereby inducing selective fluorescence enhancement. This selective fluorescence excitation (ON) and quenching (OFF) effect brought out by the synthesized theranosomes automatically empowered them to be right choice to act as label-free fluorescence bioimaging probes. In- 
addition, both the theranosomes also induced selective cancer therapy while simultaneously encouraging fibroblast cell proliferation as seen from cell viability and flow cytometry analysis (ROS and annexin/PI) conducted exclusively for validation purpose. These theranosomes were synthesized using a simple topdown multiphoton ionization mechanism and self-assembling them in a 3D fashion. The ability to fine tune the ionization mechanism realized in creating the two distinct theranosomes primary (quantum - $\mathrm{Ni}$ ) and secondary (quantum - NiO) with varying material chemistry and properties. The 3D arrangement of the quantum theranosomes combined with tunable physiochemical properties offered itself as a potential candidate in creating label-free fluorescence imaging and cancer cell therapy without any adverse sideeffects to mammalian fibroblast cells thereby demonstrating biocompatibility by mimicking a tumor microenvironment. The results accomplished and data collated in our research study here pave way for application of these magnetic quantum theranosomes to act and serve as an ideal candidate for the next generation label-free cancer theranostics. 
Label-free Fluorescence Bioimaging probes - Quantum Theranosomes
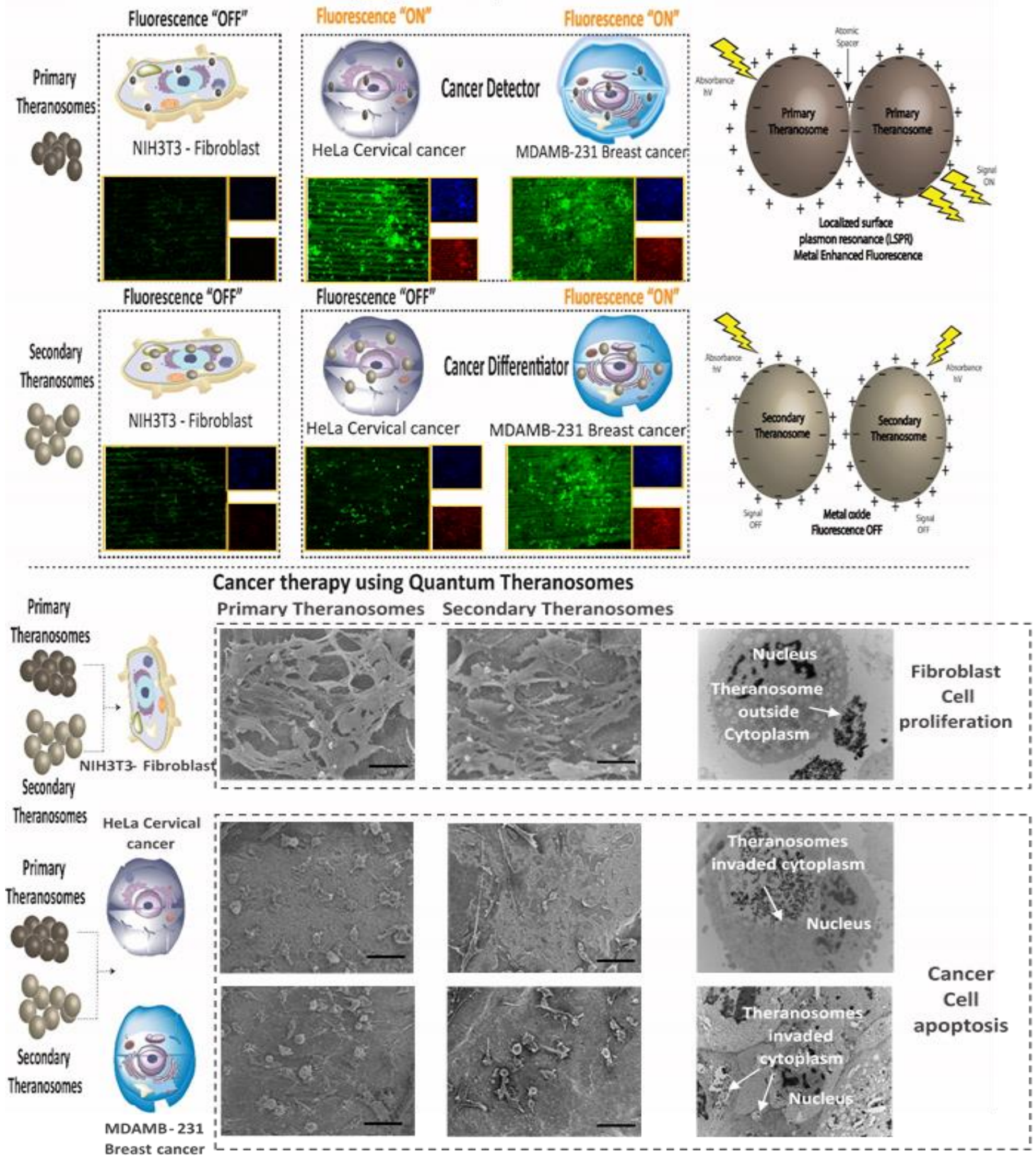

Figure 3-0: Overall schematic of Quantum theranosomes synthesis: application in Bioimaging and cancer therapy 


\subsection{Materials and Methods:}

\subsubsection{Materials:}

Nickel 200 sheet purchased from (Onlinemetals.com USA) having 99\% nickel content was cut into (3x10x15) mm (DxWxL) to be used for conducting experiments. SiC grit sandpaper (200 to 1200) was used to induce gradual mechanical polishing over the nickel surface and later was ultrasonically cleaned using $100 \%$ ethanol and acetone for a 15 -minute duration each to remove all debris particles embedded during surface polishing process. All the polished samples were then washed again with de-ionized (DI) water and finally air dried for conducting further experimentation.

\subsubsection{Laser ionization to synthesize theranosomes:}

A tunable Yb diode pumped fiber laser from Impulse series (Clark - MXR) having a central wavelength of $1030 \mathrm{~nm}$ with an average power of 16 watts was utilized towards the fabrication of the desired quantum theranosomes scale. The laser beam with a $10 \mu \mathrm{m}$ spot diameter was focused onto the nickel substrate. The ultra-short pulsed laser system with varying laser pulse repetition rate between $200 \mathrm{KHz}$ to $25 \mathrm{MHz}$ was utilized. The air-dried nickel substrates were then mounted on an $\mathrm{X}-\mathrm{Y}-\mathrm{Z}$ precision stage which is normal to the laser beam direction. To create an even distribution of quantum theranosomes on nickel substrate, a piezo-electric raster scanning system with EzCAD software was used. For the ionization of quantum theranosomes, laser pulse repetition rate (4 MHz to $26 \mathrm{MHz}$ ) along with laser pulse width (214fs) was used under gaseous medium species of $\mathrm{N}_{2}$ for the formation of the ion-plume. The gases were injected directly onto the interaction zone through several individual nozzles by continuously maintaining a flow rate of 1 Bar pressure which evenly surrounds the total core within the interaction zone.

\subsubsection{Synthesis of theranosomes by multiphoton ionization mechanism of solid nickel substrate:}

The synthesis of magnetic quantum theranosomes over the nickel substrate by ultra-short pulsed laser interaction is depicted in schematic Figure 3-0,1. A computer controlled scanner allowed for a precise two 
dimensional (2D) movement of ultra-short laser beam over the nickel substrate in a X-Y direction to synthesize 3D quantum theranosomes which is composed of both nickel and nickel oxide. This 3D quantum theranosomes generation by the ultra-short laser pulses in the presence of inert nitrogen atmospheric medium is a complex gas dynamic and a non-linear process, involving a very specific and set sequence of events: surface energy absorption of laser pulses, plasma plume ignition \& ionization, rapid condensation and ejection of species to atmosphere. In multi-photon ionization process, ultrashort laser pulses with nanosecond pulse to pulse separation time (ns) possessing microjoule $(\mu \mathrm{J})$ energy is focused onto the nickel surface, resulting in ionized species formation within the interacting plume which later cools at an ambient atmosphere to form quantum theranosomes. During this process, only a small focal volume within the plume absorbs the incident energy and transforms the material thereby minimizing thermal stress and damage. The control over the state of this expanding plume is based on a combination of various parameters such as laser pulse energy $(\mu \mathrm{J})$, pulse interaction time (fs) and peak power (MW) of incoming laser pulses. Here, the energy deposited by ultrashort laser pulses far exceeds the inter-atomic binding energy level and the atom under excitation becomes totally free from its bonds only to interact further with the incoming ultra-short laser pulses. This initiates a kinectic reaction in an explanding plume to stay in a gaseous-ionic state encompassing non-interacting atoms ejected from the interacting material surface onto interact further with an oncoming laser pulses impinged when in ambient atmosphere. Since laser pulse interaction time is in the order of magnitude lower than material heat diffusion time, it induces superheated ion formation reaction creating ionised species which transforms material from one crystalline structure to an another. This very swift transformation from the initial cold solidified state to an ionic state of the substrate when under excitation causes a time-dependent ionization and material recombination process. The final ejection of the ionised speciecs coming out from the plasma plume forces the superheated ions to cool down as it propels further away from the interacting surface only to recombine with the oxygen medium present in the ambient atmosphere thereby condensing to form a 
3D self assembled quantum scale $\mathrm{NiO}$ and $\mathrm{Ni}$ particles. The growth rate of every individual particles varies with both lattice plane orientations and temperature gradient, thereby enabling effective control over both the particle morphology and its size.

\subsubsection{Theranosomes morphological and physiochemical characterization:}

The synthesized quantum theranosomes specimens were imaged using a high-resolution scanning electron microscope (HRSEM). The HRSEM/EDX attachment from Oxford Instruments was used to analyze the elemental composition of the synthesized theranosomes. To quantitatively identify the oxide composition of the synthesized theranosomes an X-ray Diffraction (Phillips powder XRD) was employed with $\mathrm{Cu}$ K- $\alpha$ radiation $\left(\lambda=1.54054 \AA\right.$ ) ) at $40 \mathrm{KV}$ and $40 \mathrm{~mA}$ with a $2 \theta$ scanning speed of $0.010^{\circ} \mathrm{min}^{-1}$. Subsequently Reitvield analysis was also carried out on the acquired spectrum. Thereafter, Bruker Senterra Dispersive Raman Microscope fitted with a 532-nm laser system was used to analyze the theranosomes. A 50X magnification objective was used to focus the laser onto the sample surface with an appropriate laser irradiation power of $5 \mathrm{~mW}$. A standard collection time of $10 \mathrm{~s}$ with three iterations was employed and all the necessary parameter were chosen by an earlier trial and error method conducted to reduce the spectrum noise. All the spectrums collected were baseline corrected and normalized to laser power.

\subsubsection{Optical characterization:}

A colloidal suspension of the theranosomes synthesized was made using a Phosphate Buffer Solution (PBS) and a Hitachi UV-3100 UV-vis-NIR spectrophotometer was used to analyse UV-vis-NIR absorption and reflection spectrums of synthesized primary and secondary theranosomes.

\subsubsection{In-vitro Cell culture:}

HeLa, human cervical cancer cell line, was obtained from ATCC (American type culture collection, ATCC No. $\mathrm{CCl}-2$ ) and were cultured in DMEM-F12 medium with phenol red containing heat inactivated fetal 
bovine serum and $1 \%$ penicillin-streptomycin antibiotics at $37^{\circ} \mathrm{C}$ in a $5 \% \mathrm{CO}_{2}$ atmosphere. NIH3T3 fibroblast cells and MDAMB -231 were also obtained from ATCC were in DMEM medium containing $10 \%$ fetal bovine serum with $1 \%$ penicillin-streptomycin antibiotics at $37^{\circ} \mathrm{C}$ in $5 \% \mathrm{CO}_{2}$. The theranosomes are washed with alcohol and DI water and then kept under UV light for a duration of 15 minutes. The substrates are then placed in separate petri-dishes with HeLa, MDAMB-231 and NIH3T3 cells are seeded individually to attain a density of $10^{5}$ cells $/ \mathrm{ml}$, totaling to $4 \mathrm{ml}$ volume by quantity per dish. The petri dishes are then placed in an incubator for 24 and 48 Hours.

After the set incubation period, the samples are fixed for SEM imaging in a $2 \%$ glutaraldehyde over a 0.1 M sodium cacodylate buffer of $\mathrm{pH} 7.3$ for an hour. Later, the samples are immersed in $0.1 \mathrm{M}$ cacodylate buffer with $0.2 \mathrm{M}$ sucrose for about 20 minutes. The samples are then dehydrated in an increasing concentration level of alcohol for 20 minutes duration each time. The samples are then critical point dried and gold sputtered for achieving improved imaging. SEM imaging was conducted using (Hitachi SU1500) at $10 \mathrm{kV}$ with a magnification range between 500 to 15000 times. Three separate images were chosen for each of the condition to further conduct the quantitative analysis for measuring cell analysis using ImageJ software.

\subsubsection{Label-free Fluorescence Bioimaging:}

The samples are prepared very similar to the in-vitro cell culture process and after the set incubation period of 24 hours, the samples are washed once using 1\% PBS and there after fixed using $4 \%$ methanol free paraformaldehyde. The samples are then air-dried and made ready for bioimaging using epifluorescent Nikon E-400 microscope with 3 filter setting (DAPI, FITC and Texas Red). The data were recovered by DS-5M-UI color digital camera (Nikon, Canada) and collated. 


\subsubsection{Differential fluorescence staining of cells:}

The samples were seeded with appropriate cells and incubated for 24 hours and 48 hours similar to the SEM sample investigation. After the set incubation period, all the samples were fixed in $4 \%$ methanol free paraformaldehyde followed by an incubation in a $1 \%$ skimmed milk to prevent non-specific binding. To stain the actin cytoskeleton, the samples were incubated with Alexa flour 488 (Life Technologies) followed by DAPI (4', 6'-diamidino-2-phenylindole, Life Technologies) to stain the nucleus. The samples are then air dried and later imaged using an epi-fluorescent Nikon E-400 microscope. The data were recovered by DS5M-UI color digital camera (Nikon, Canada) was collated.

\subsubsection{Assessment of necrosis and apoptosis using Annexin V-PI staining:}

Apoptosis in HeLa, MDAMB-231 and NIH3T3 cells was measured by using the Annexin V-Propidium lodide (PI) and apoptosis detection kit (BD Biosciences, San Diego, USA). All the cells were exposed to primary (quantum-nickel) and secondary (quantum-nickel oxide) theranosomes in a 6-well plate for a duration of 24 and 48 hours. After the said exposure, cells were first trypsinized and later centrifuged at $1000 \mathrm{rpm}$ to form pellets which were all washed with PBS once and again re-suspended in $1 \mathrm{~mL}$ of binding buffer, later incubated with $0.2 \mathrm{~mL}$ Annexin V-FITC for $10 \mathrm{~min}$, followed by staining with $0.2 \mathrm{~mL}$ PI. Thereafter, the samples were then analyzed with a Fortesse X-20 flow cytometer from (BD Bioscience- St. Michael's hospital), and at least a minimum of 10,000 cells were counted for each sample. The cell population of interest was gated based on the forward and side-scatter properties. Both the vertical and horizontal lines are designed based on auto fluorescence of untreated control cells. The different labeling patterns in the Annexin V/PI analysis adopted was to identify the different cell populations wherein both the FITC negative and the PI negative are designated to be as termed viable cells; FITC positive and PI negative cells were earmarked as early apoptotic cells; FITC positive and PI positive cells were marked as late apoptotic cells; and FITC negative and PI positive cells as necrotic cells. The complete data analysis was performed using BD FACS Diva software. 


\subsubsection{Reactive oxygen species (ROS)determination using flow cytometry and fluorescence microscopy:}

Cells were all treated with QS theranosomes for a duration of 24 and 48 hours duration. The cells were then first harvested by trypsinisation process and thereafter centrifugated at $1000 \mathrm{rpm}$ to form pellets. The pellets were resuspended in DCFDA working solution for a duration of $30 \mathrm{~min}$ at $37^{\circ} \mathrm{C}$. After washing with PBS, the pellets were once again resuspended in PBS to be immediately analyzed using flow cytometry (Fortessa X-20) BD bioscience. A minimum of 10,000 events were recorded and the data was processed using BD FACS Diva software. Similarly, cells were treated with primary and secondary theranosomes for 24 and 48 hours and cell intracellular ROS generation was determined by fluorescence microscopy probe DCFH-DA. The green fluorescence intensity of DCF was found enhanced in the theranosomes treated cells when compared with the control cells.

\subsubsection{Transmission Electron Microscopy - Bio TEM:}

Theranosomes treated (MDAMB-231, HeLa and NIH3T3) cells were pelleted ( $1 \times 10^{6}$ cells/pellet), fixed in $2 \%$ glutaraldehyde in $0.1 \mathrm{M}$ sodium cacodylate buffer and stored at $4{ }^{\circ} \mathrm{C}$ until sectioning. To prepare the TEM grids, cells were rinsed in buffer and fixed in $1 \%$ osmium tetroxide in buffer, dehydrated in a graded ethanol series followed by propylene oxide, and embedded in EMBed812 resin. Cells were then stained with uranyl acetate and lead citrate. $100 \mathrm{~nm}$ thick sections were then cut using an RMC MT6000 ultramicrotome and viewed in an FEI Tecnai 20 TEM. TEM preparation and imaging were performed at the Nanoscale Biomedical Imaging Facility of the Hospital for Sick Children Research Institute, Toronto, Canada.

\subsubsection{Statistics:}

All experiments were carried out in triplicates and all data points collected were standard mean with \pm standard error unless otherwise mentioned. The error bars provided also indicate standard deviation. On 
the whole an overall 95\% confidence interval for the mean was achieved.

\subsection{RESULTS AND DISCUSSION:}

\subsubsection{Morphological and material chemistry investigation of theranosomes:}

The surface morphology of the self-amassed 3D quantum scale theranosomes synthesized under nitrogen atmospheric conditions indicated in Figure 3-1 was observed using FE-SEM and HR-SEM. A web consisting of both coalesced cubical and spherical like structures were noticed. These coalesced particles were further aggregated to form intertwined web like structure which were arranged over multiple layers to form the 3D quantum theranosomes. The morphology of the synthesized theranosomes at nitrogen filled atmosphere was analyzed and was observed to have a wider size distribution of particles as can be seen from graphs in Figure 3-1. Based on the above observation, the synthesized particles within the observed structure is then further characterized into primary (quantum-Ni) and secondary (quantum-NiO) theranosomes respectively. To study the morphological particle distribution range of these synthesized theranosomes, a HR-TEM analysis was carried out. The distribution graphs from Figure 3-1 show particle size histogram performed over several observed images. The results fit into a single log-normal distribution which describe the most prominent features of the histogram. The mean distribution size of primary and secondary theranosomes particles were $4.7 \mathrm{~nm}$ and $8.8 \mathrm{~nm}$ respectively. 

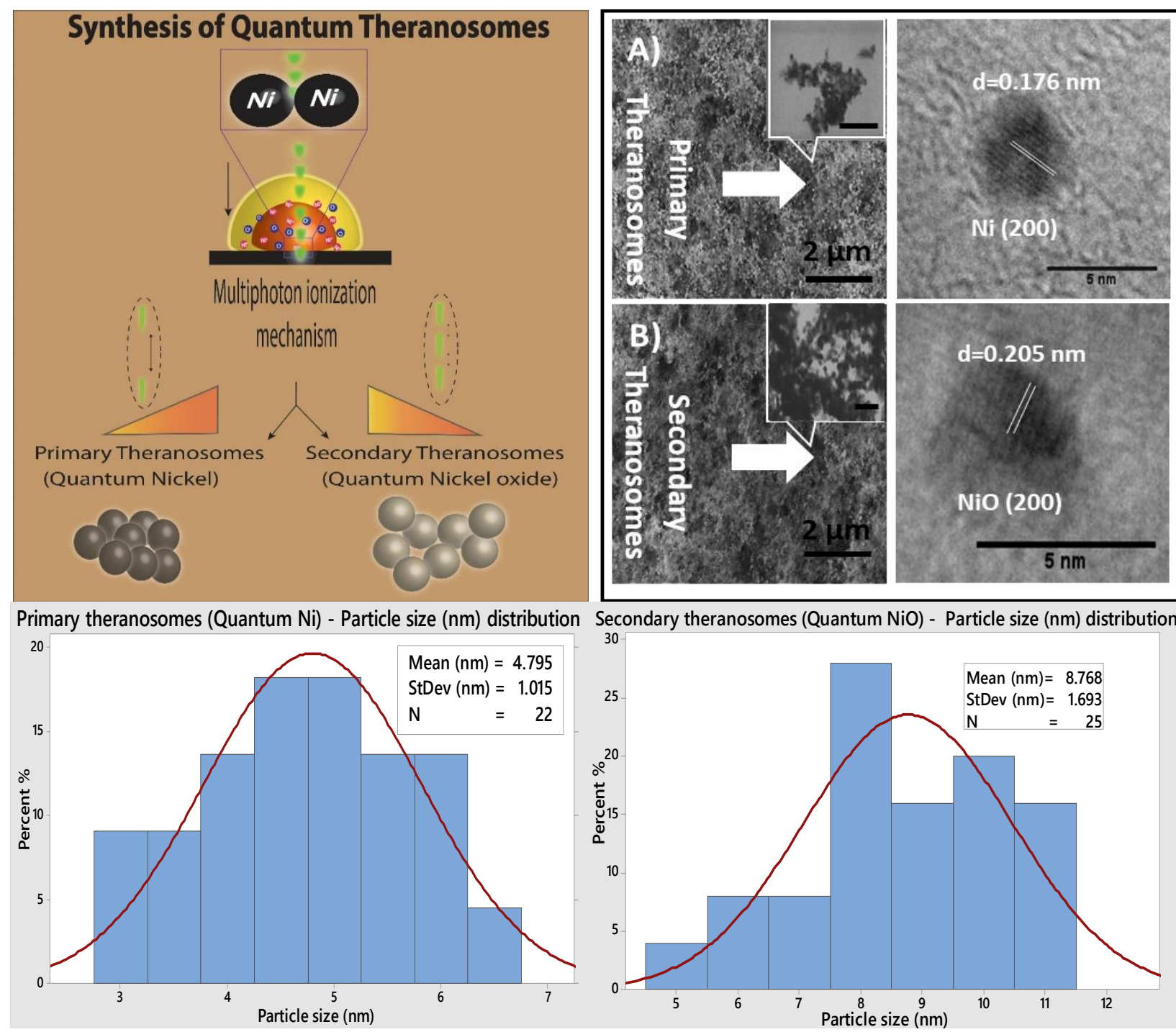

Figure 3-0-1: Characterization of primary (quantum Ni) and secondary (quantum NiO) theranosomes. The FESEM shows the self-assembled $3 D$ quantum structures. The HR-SEM insets shows individual particle morphology which are composed of primarily spherical and cubic particles. HR-TEM displays lattice spacing ' $d$ ' indicating the presence of Nickel and Nickel oxide along with particle size distribution

The surface characterization of these synthesized theranosomes was performed using Raman, XRD and XPS to determine the change in the chemical composition and the different oxidation states of primary (quantum-Ni) and secondary (quantum-NiO) theranosomes. For all the above conditions, we observed that the Raman bands from $\mathrm{Ni}$ and $\mathrm{NiO}$ were in the spectral region above $400 \mathrm{~cm}^{-1}$. The Raman peaks noted in Figure 3-2(A, B) are primarily vibration modes due to the first and second order Raman scattering caused by phonons in $\mathrm{Ni}$ and $\mathrm{NiO}$ whereas, the band above $1200 \mathrm{~cm}^{-1}$ originates due to scattering by two 
magnons in $\mathrm{Ni}$ and $\mathrm{NiO}$. In general, Magnons are observed in magnetic materials due to the electron spin waves excitations. These inelastic scattering observed with magnons can also occur in magnetic materials due to spin-orbit coupling ${ }^{97}$. The phonon and magnon modes were analyzed quantitatively by deconvoluting Raman spectra using Gaussian functions. The intense Raman band observed in Figure 3-2A at $\sim 509 \mathrm{~cm}^{-1}$ was assigned to the first-order one phonon (1P) LO mode. The Raman band observed near $500 \mathrm{~cm}^{-1}$ could also be of magnetic origin. In the present work, the Raman bands positioned at $\sim 509 \mathrm{~cm}^{-1}$ could be directly related to quantum scale particle (nanocrystallites) as particle size influences magnetic behavior $^{98}$. Also, there was total absence of the first order TO mode under all conditions. We then assigned the $1075 \mathrm{~cm}^{-1}$ band to the second order LO phonon modes. The relative intensity of LO phonon mode was found enhanced with primary theranosomes (quantum-nickel) when compared with secondary theranosomes (quantum-nickel oxide) relating to change in particle size variation as cited in literature ${ }^{98}$. In addition, the decreasing peak size at secondary theranosomes indicate a high level of nickel vacancy in the synthesized theranosomes. Furthermore, second order (2TO) transverse optical mode of NiO was observed at all theranosomes at $709 \mathrm{~cm}^{-1}$ as seen from Figure 3-2A. Moreover, the two magnon modes (2M) at $\sim 1400 \mathrm{~cm}^{-1}$ intensity was observed only at primary theranosomes (quantum- nickel). From the literature and previous studies, it was presumed that $2 \mathrm{M}$ vibrations seen in Nickel based nanomaterials are primarily due to antiferromagnetic super exchange interaction in nickel ions and that in the linear atomic chain $\mathrm{Ni}^{2+}-\mathrm{O}^{2-}-\mathrm{Ni}^{2+62,99}$. The near and complete absence of $2 \mathrm{M}$ excitations seen in Figure 3-2A at secondary theranosomes (quantum - nickel oxide) was consistent with the enhancement of LO mode conditions. This directly refers to the even of bond breakage of $\mathrm{Ni}^{2+}-\mathrm{O}^{2-}-\mathrm{Ni}^{2+}$. Furthermore, the $3 \mathrm{MO}$ mode at $\sim 2300 \mathrm{~cm}^{-1}$ was present in all conditions. In addition, the observed first-order Raman band was derived from symmetry-breaking imperfections and defects like (Ni vacancies, or disorder in crystal lattices), hence it can be suggested that its FWHM can be used as an indicator for the presence of these defects in the $\mathrm{NiO}$ samples. 

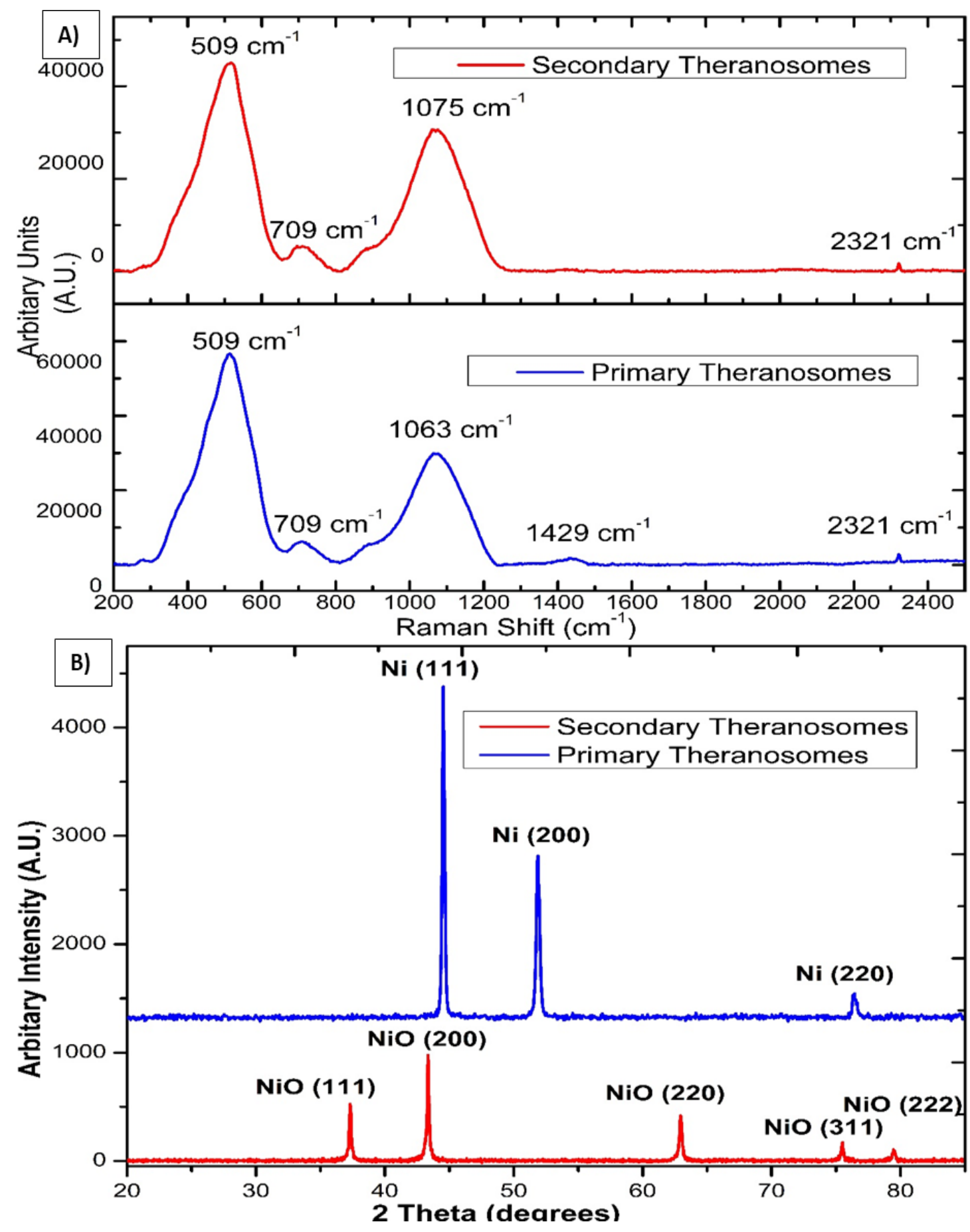

Figure 3-0-2: A) Raman spectra of primary (quantum-Ni) and secondary (quantum-NiO) theranosomes B) X-Ray diffraction and reitvield analysis of the synthesized theranosomes to qualitatively identify weight percentage of observed phases 
Additionally, X-ray diffraction (XRD) analysis of the synthesized quantum scale theranosomes in Figure 32B shows a significant line broadening which was characteristic of nickel and nickel oxide. The reflection peaks for nickel was indexed to face-centered cubic (fcc) structure of nickel. Three diffraction peaks at $2 \boldsymbol{\theta}$ $=44.5,51.9,76.5$ correspond to the Miller indices $\{111,200,220\}$ facets of primary (quantum - Ni) theranosomes. The peaks at $2 \boldsymbol{\theta}=37.28,43.28,62.88,75.28,79.48$ correspond to the diffraction from $\{111$, $200,220,311,222$ facets of secondary (quantum - NiO) theranosomes ${ }^{100,101}$. The above results were also confirmed with HR-TEM lattice images of Primary and secondary theranosomes as seen from inset Figure 3-1. Reitvield fitting was conducted on the acquired spectrum to derive a qualitative weight percentage approximation of each identified phases in the synthesized theranosomes. Furthermore, XPS was deployed as a qualitative technique to establish and resolve the chemical and surface atomic composition of the synthesized theranosomes.

The survey scan results are presented in the Appendix Figure B-S1(A\&B) which exhibits the main corelevel peaks for $\mathrm{O} 1$ sand $\mathrm{Ni2}$ p centered at binding energies (BEs) 532, and $855 \mathrm{eV}$, respectively. The binding energy (BE) scale was corrected with reference to the spurious C1s peak (282.9 eV) attributed to the specimen charging and surface contamination effects. Detailed energy analysis of the Ni2p (Figures $3 \mathrm{~A}$ ) spectrum shows its characteristic spin-orbit splitting of the nickel ion into $2 \mathrm{p} 3 / 2$ and $2 \mathrm{p} 1 / 2$ peaks (at 856.38 and $874.08 \mathrm{eV}$ ), respectively with an energy separation of $17.7 \mathrm{eV}$. The corresponding satellite peaks observed at higher energies $862 \mathrm{eV}$ and $880 \mathrm{eV}$ relate to $2 \mathrm{p} 3 / 2$ and $2 \mathrm{p} 1 / 2$ energy region. These satellite peaks at higher energies more than the " $2 \mathrm{p}$ " principal peaks in this energy region have been ascribed to a charge transfer multielectron transition ${ }^{102}$. These features corroborate to suggest that the surface oxidation of the primary (quantum - nickel) theranosomes, even though with their low intensity profile is indicative of a smaller extent of the oxidation phase that it had undergone. In-addition, the intensity of the Nickel peaks noted was significantly higher at primary (quantum nickel) theranosomes when compared with secondary (quantum nickel oxide) theranosomes. 

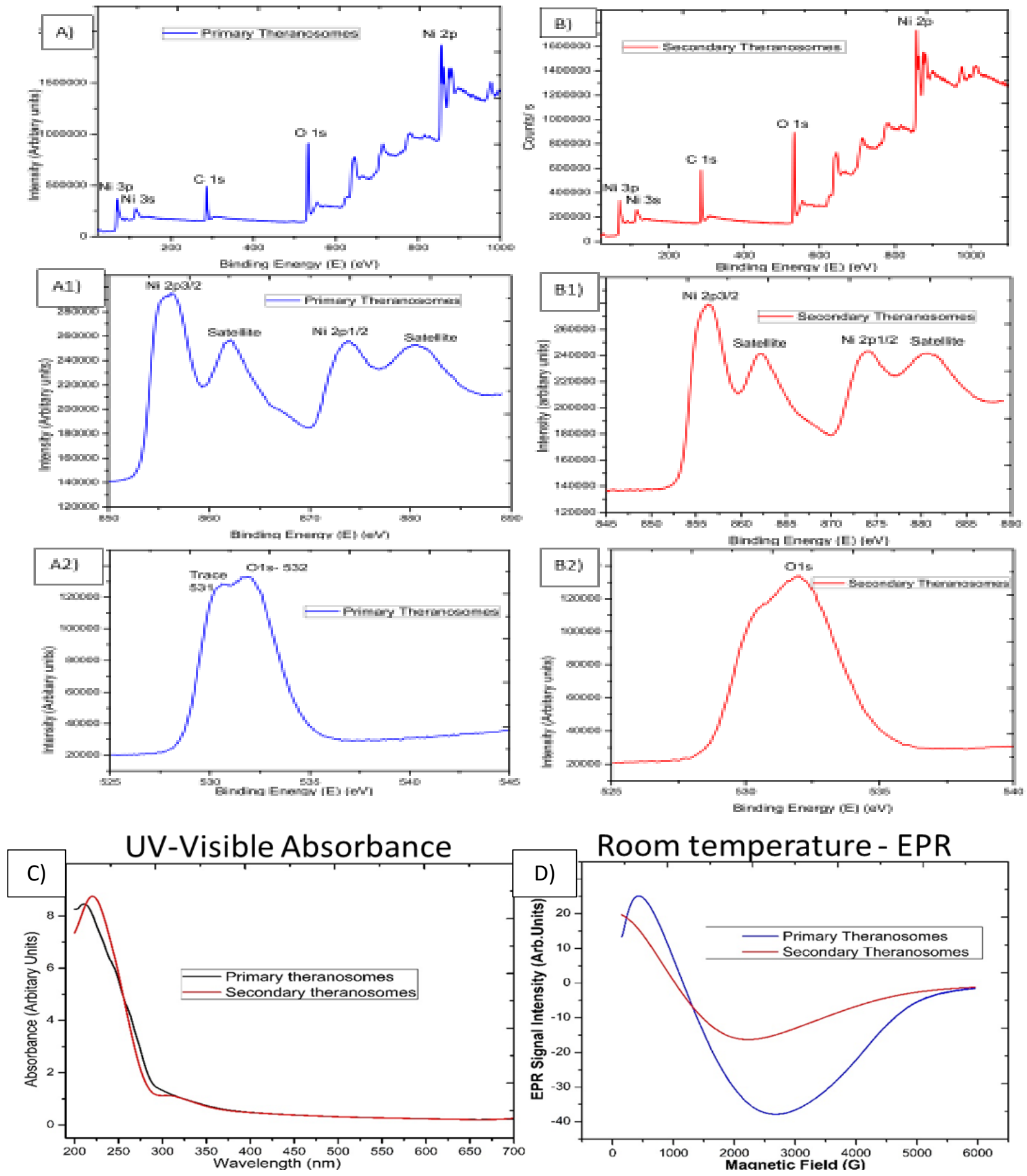

Figure 3-3: XPS survey and individual spectrum exhibiting strong presence of nickel and oxygen at A) Primary Theranosomes and B) Secondary Theranosomes C) UV-Vis spectra of theranosomes exhibiting increased photon absorption D) Room temperature electron paramagnetic resonance curves for different theranosomes 
Furthermore, the individual scans, showed the presence of trace oxygen content and strong O1s core level spectrum signal from the primary (quantum - Nickel) ${ }^{103}$. However, only a strong O1s core peak was apparent from the secondary (quantum - nickel oxide) theranosomes as observed in Figure 3-(3A and 3B).

\subsubsection{Optical(UV-Vis) and Magnetic (Resonance Properties using EPR) characterization:}

The synthesized quantum theranosomes were first investigated with ultraviolet-visible absorption at room temperature, ranging from 200 to $700 \mathrm{~nm}$ exhibited a significant high photon absorption as shown in figure 3C. It was evident from the synthesized theranosomes that the absorption level gradually decreases with an increased of wavelength from 250 to $700 \mathrm{~nm}$. The absorption band was observed at $212 \mathrm{~nm}$ for primary (quantum - Ni) theranosomes and $220 \mathrm{~nm}$ for secondary (quantum - NiO) theranosomes. Bulk nickel nanomaterial absorption band was centered around $230 \mathrm{~nm}$ as noted and cited from the available literature ${ }^{104}$. The observed red shift in the absorption bands in our present investigation was due to the quantum size confinement effect with (quantum-Ni) primary theranosomes particles shifting more than the (Quantum-NiO) secondary theranosomes thereby confirming the presence of nickel and nickel oxide particles ${ }^{105,106}$. In-addition, metallic nickel has no band gap and the observed UVabsorption spectrum of primary theranosomes was due to the oxidation reaction of nickel to $\mathrm{PBS}^{107}$.

Furthermore, to investigate the magnetic properties, Electron Paramagnetic Resonance (EPR) spectroscopy was used to study the synthesized materials states with unpaired electrons. It was observed that the primary (Quantum-Ni) and secondary (Quantum-NiO) theranosomes in figure 3-3D exhibit a narrow resonant peak at low magnetic field. The relative peak position of the absorption lines depends on the particle size. i.e., the peak position shifts to higher fields indicating that the material is in a ferromagnetic state. It can now be well acknowledged that EPR signal of NiO disappears because of the presence of strong exchange fields. On the other hand, particle size which was less than $100 \mathrm{~nm}$ can also exhibit paramagnetism when at room temperature due to the presence of $\mathrm{Ni}^{+}$ions already present in $\mathrm{NiO}$. 

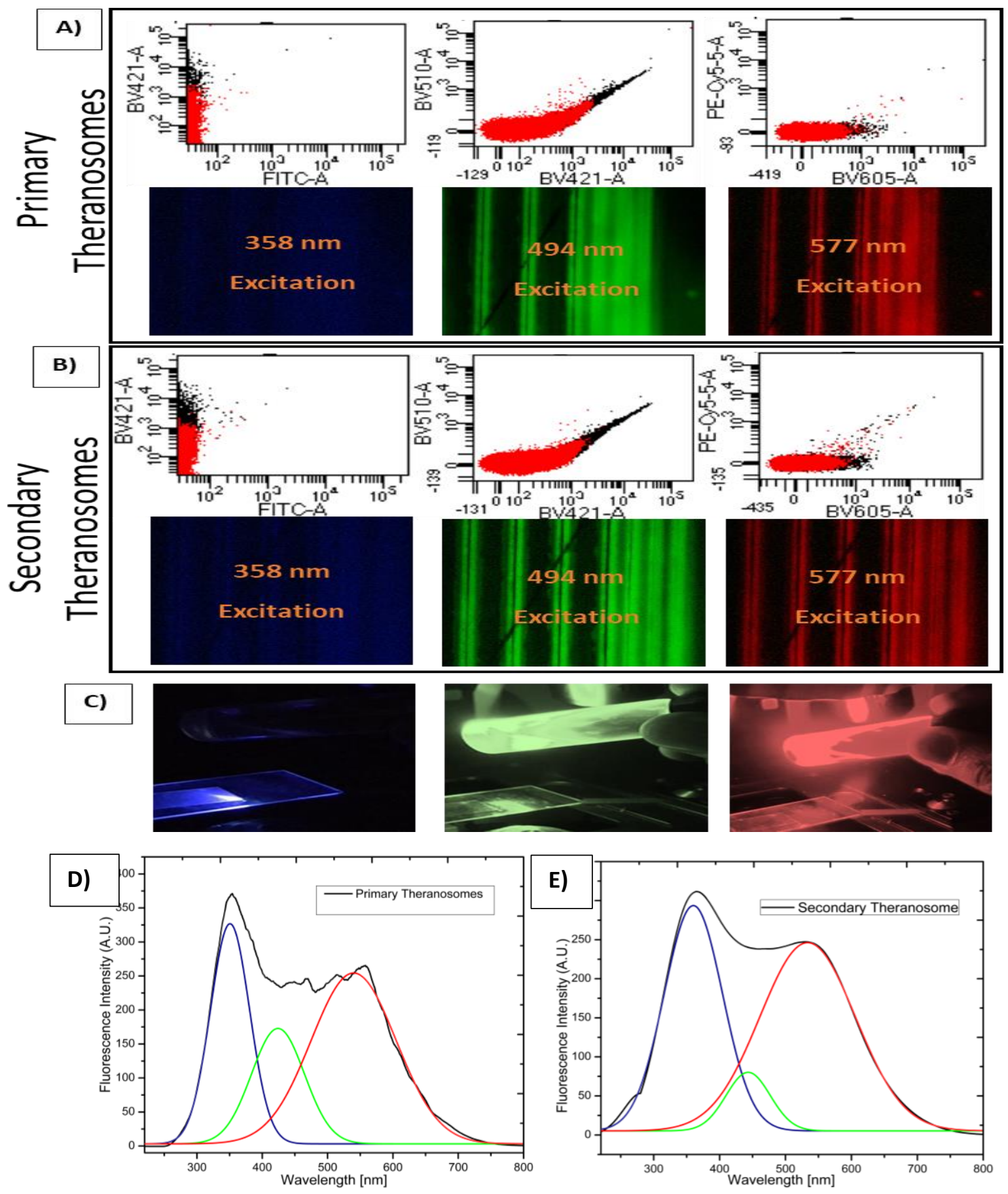

Figure 3-4: Emission of theranosomes at excitation wavelengths of multiple channels at 358, 494,577 $\mathrm{nm}$ exhibits the fluorescence characteristics of the theranosomes using flow cytometry. A comparison fluorescence intensity at $A$ ) primary theranosomes and $B$ ) secondary theranosomes $C$ ) $A$ representative comparison shows the different fluorescence intensity of the synthesized colloidal theranosome particles under corresponding fluorescence excitation channels in fluorescence microscopy. Photoluminescence (PL) synchronous spectrum of D) Primary and E) Secondary theranosomes showed strong PL with respect to the excitation wavelength. The emission was at a broadband visible wavelength which demonstrates fluorescence excitation of theranosomes 
Ulmane et al., and Gandhi et al., did extensive study on size dependent properties of nickel based nanomaterials. Based on their observations, the decreasing and complete absence of $2 \mathrm{M}$ mode from the Raman spectrum with increasing LO mode was correlated to the surface disorders of $\mathrm{NiO}^{108,109}$. In our present study, the formation of EPR signal when in comparison with the magnetic properties could be attributed to the existence of uncompensated spin on the surface of the particles, which provides sufficient supportable explanation for the presence of these field dependent magnetic properties.

\subsubsection{Quantum Theranosomes fluorescence enhancement intensity using Flow Cytometry and} fluorescence microscopy assessment:

To assess the potential as a bioimaging probe, we examined the quenching and excitation effect of the synthesized quantum theranosomes which were made into colloidal suspensions using phosphate Buffer solution (PBS) and were analyzed using flow cytometry by acquisition of the side-scattering (SSC) and forward-scattering (FSC) signal from figure 3-4. A notable change in SSC and FSC signals was revealed in the suspension when compared with control where the theranosomes showcased increased activity in all the visible channels in both forward and side scattering channels. This is backed up by the photoluminescence (PL) spectrum of the synthesized theranosomes presented in figure 3-4(D\&E). It confirmed the broadband emission in the visible wavelength ${ }^{106}$. The fluorescence intensity showed an increased excitation, when shifting from $400 \mathrm{~nm}$ to $700 \mathrm{~nm}$ and then gradually decreased excitation with the red shift. Moreover, the irradiation of quantum theranosomes in the visible range, as depicted in fluorescence images demonstrate higher excitation of these quantum theranosomes when viewed from the bright field, and the single (DAPI, FITC and Texas Red) channel images in figure 3(4A and B) and the Appendix Figure B - S1. 
Primary theranosome

(Fluorescence ON - cancer cell)

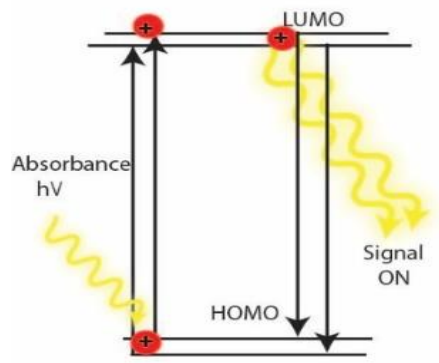

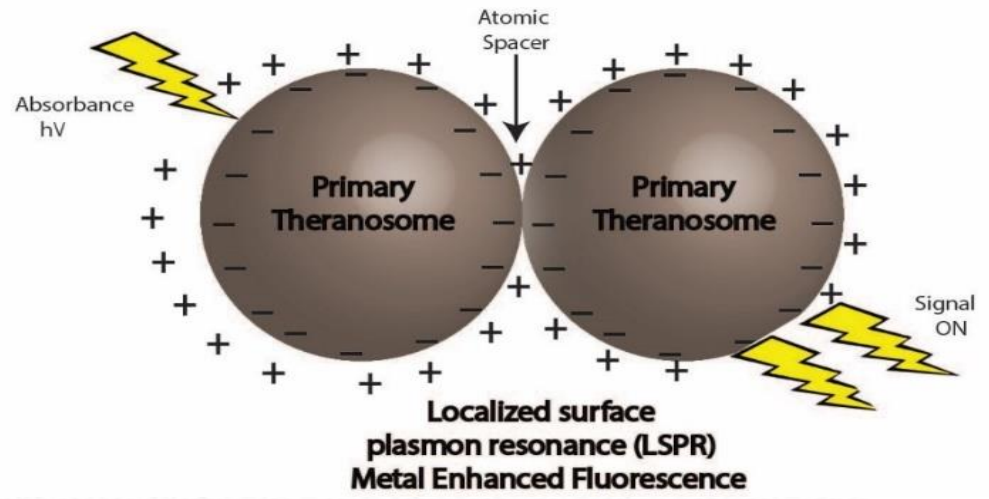

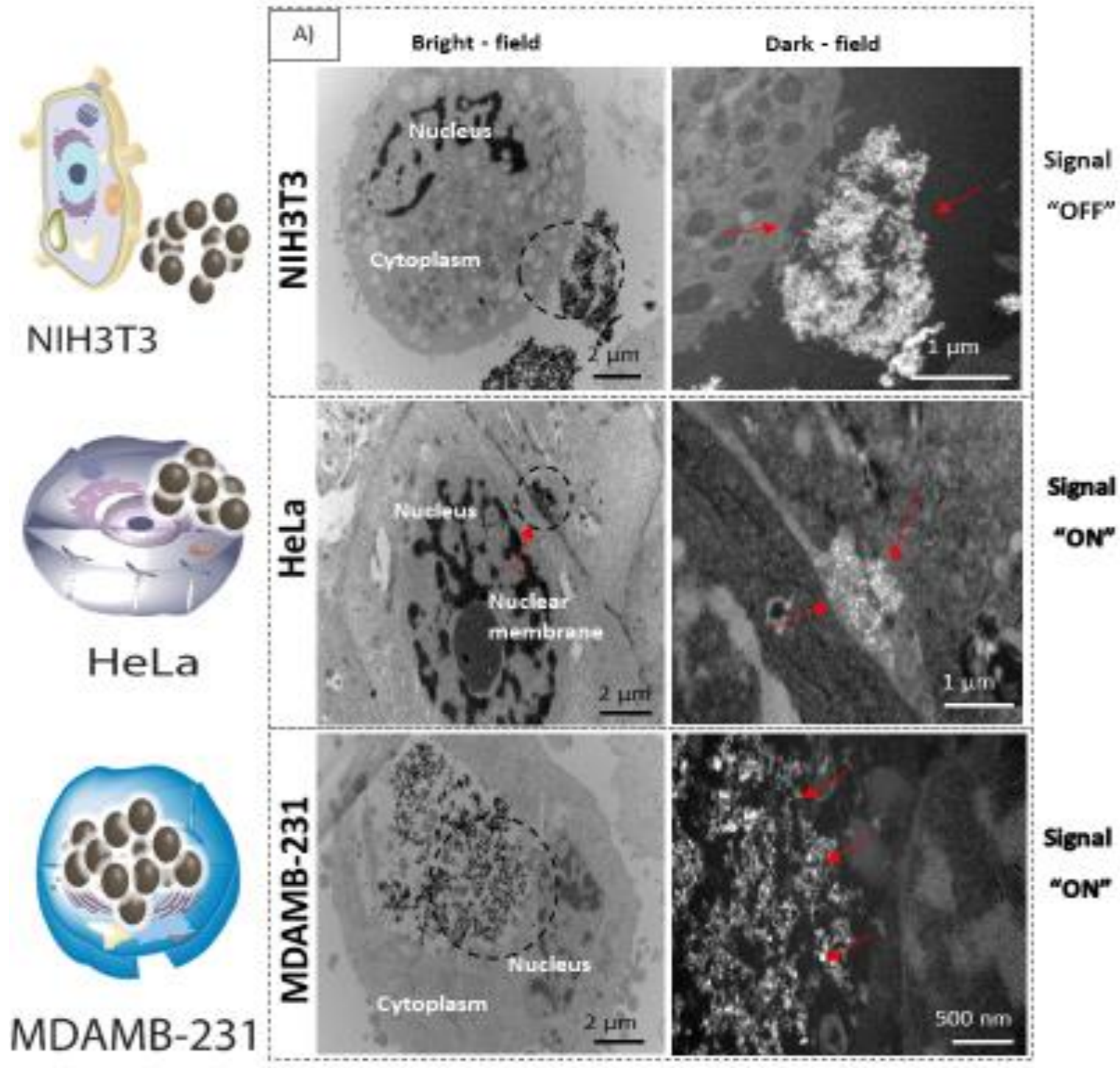

5A:

Figure 3-5A: Representative TEM image of Primary theranosomes in HeLa, MDAMB-231 and NI3T3 fibroblast cells. The theranosomes are located within the cellular membrane in both cancer cells when compared with fibroblast cells. The corresponding Dark-field images on the right show the location and localization of theranosomes 


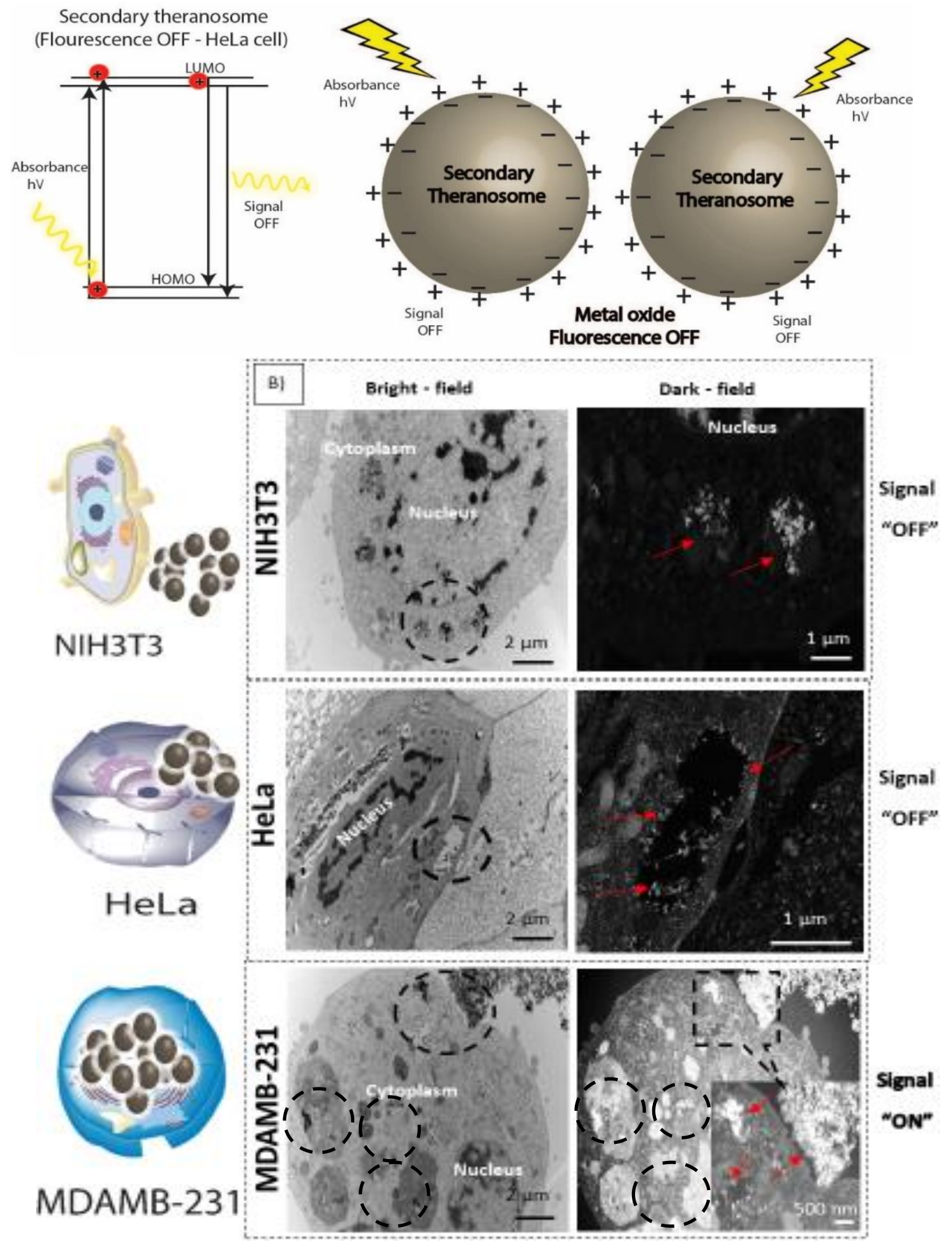

Figure 3-5B: Representative TEM image of Secondary theranosomes in HeLa, MDAMB-231 and NIH3T3 fibroblast cells. The theranosomes are located within the cellular membrane in both cancer cells when compared with fibroblast cells. The corresponding Dark-field images on the right show the location and localization of theranosomes 
To evaluate the fluorescene property and showcase nanomaterial interaction in biological environment multiple techniques have been developed, such as confocal Raman mapping, atomic force microscopy, TEM and fluorescence microscopy. TEM being extremely high resolution ( $1 \mathrm{~nm}$ ) is valuable providing both visualization of nanoparticle location in cellular compartments and nanoparticle aggregation ${ }^{110}$. Herein, TEM in used to both showcase theranosome aggregation inside the cells and demonstrate its fluorescence bioimaging property. The traditional TEM bright field mode is used allowing nanometer scale details and visualization of theranosomes in biological milieu. Since, the theranosome are inorganic in nature with high crystallinity and mass, they appear dark in the bright field mode. Thus, a dark field mode is also applied to showcase the theranosome location and activity. The synthesized theranosomes being fluorescent in nature appear as bright white spots when visualized in dark-field mode.

\subsubsection{In-vitro fluorescence bioimaging property of theranosomes:}

For cellular diagnosis, the fabricated quantum theranosomes was introduced to NIH3T3 fibroblasts, (MDAMB-231) breast cancer cells and cervical cancer cells (HeLa) as model cell lines for examining the cell viability. The cell viability from figure 3-5, 6(A, B and its corresponding insets) and Appendix B Figure S2 indicated that the quantum theranosomes are all biocompatible for conducting the analysis of living cells thereby confirming its use as a bioimaging probe. To establish the theranosomes are viable broadband fluorescence bioimaging probes, the synthesized probes must become localized in the cell and be able to distinguish normal cells from those of the cancerous cell. Yallapu et al., suggested increased intracellular uptake or accumulation of drugs and drug coated nanoparticles in cancer cells is often used as an indicator for a higher therapeutic index ${ }^{111}$. Thus, we investigated the uptake of both primary and secondary theranosomes using TEM evaluation. Bio-TEM observations on the ultrathin sections of HeLa, MDAMB231 and NIH3T3 cells after being treated with theranosomes for 24 hours is visualized in figure 3-5(A, B). This was used to explore the process of cellular uptake for cancer therapy and the use of theranosomes for fluorescence bioimaging. 

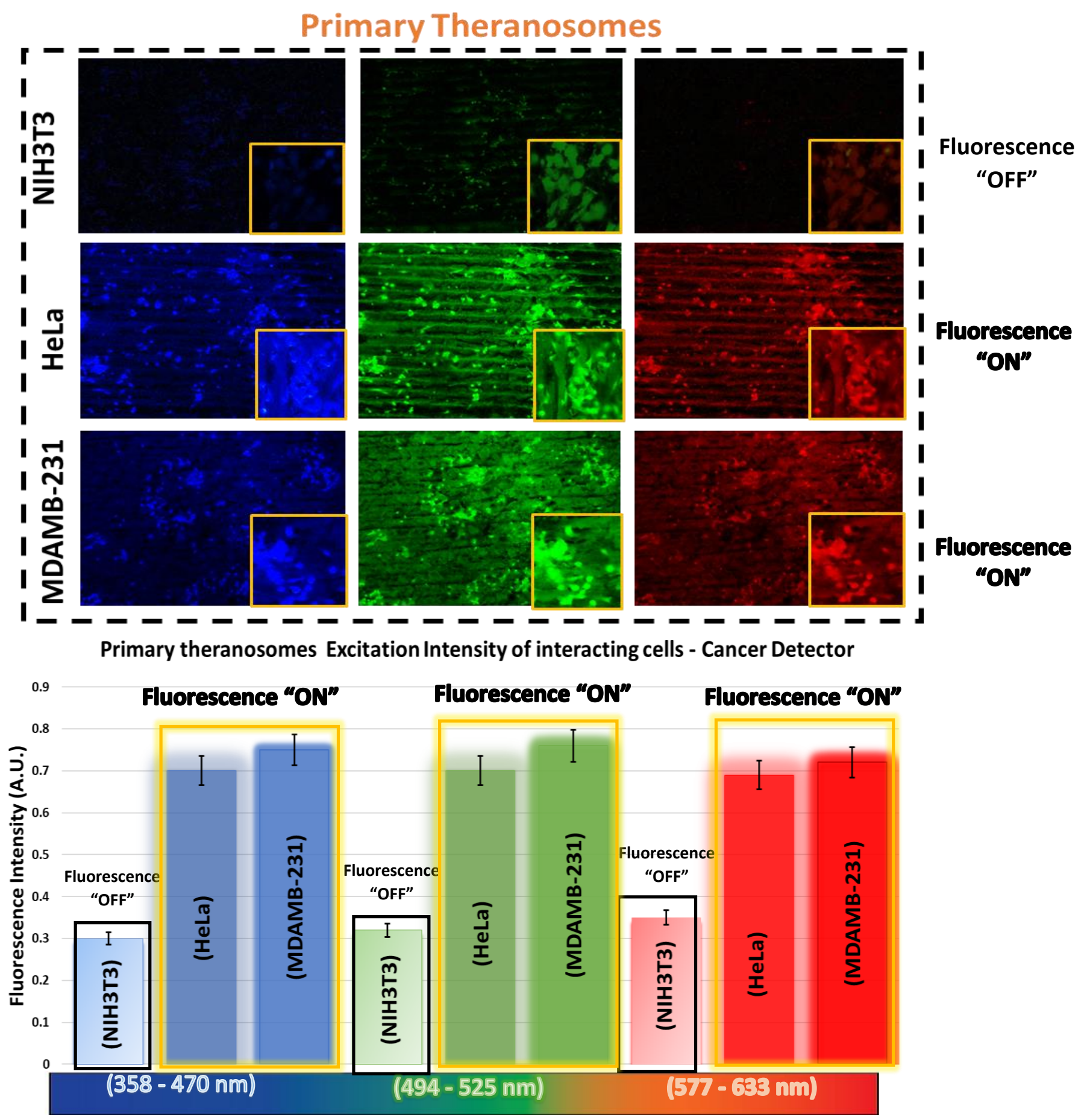

Broadband Excitation Channels

Figure 3-6A: Primary Theranosomes acting as cancer detector demonstrating increased fluorescence intensity of cancer (MDAMB-231, HeLa) cells when compared with fibroblast (NIH3T3) cells. A standard scale of $10 \mu \mathrm{m}$ and $\mathbf{5 0} \mu \mathrm{m}$ for insets are deployed 


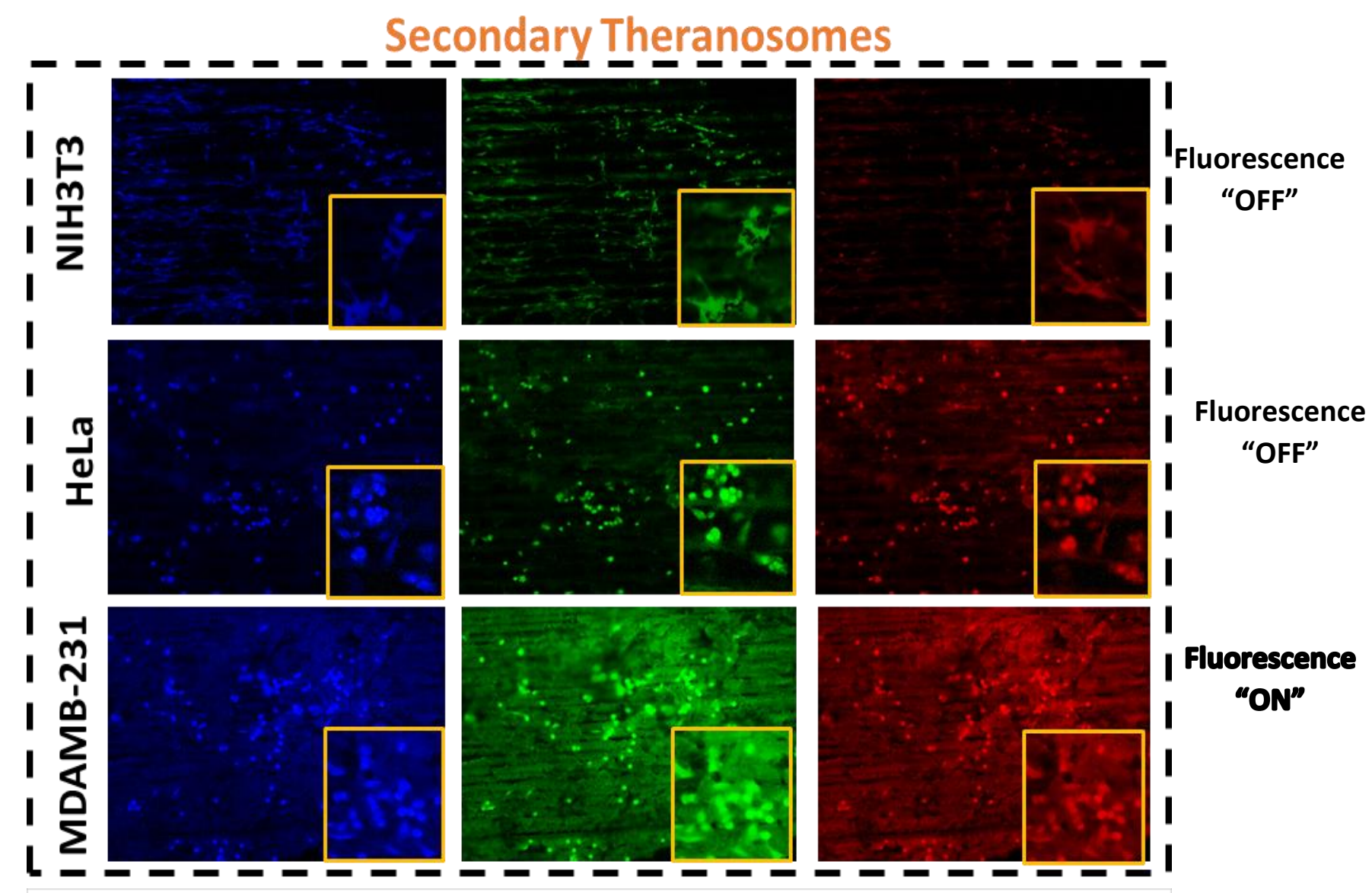

Secondary theranosomes Excitation Intensity of interacting cells - Cancer Fluorescence “ON" Differentiator

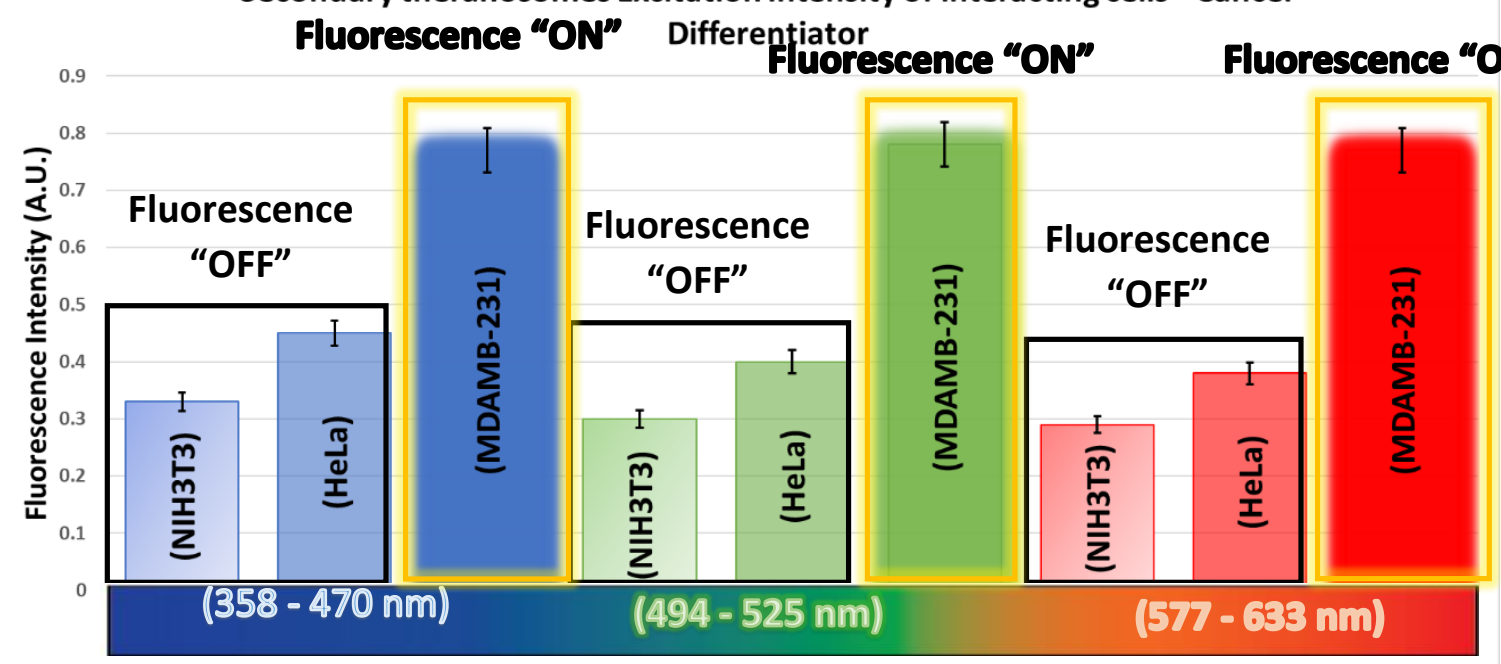

Broadband Excitation channels

Figure 3-6B: Secondary Theranosomes acting as cancer differentiator demonstrating increased fluorescence intensity of cancer (MDAMB-231) cells when compared with both fibroblast (NIH3T3) and HeLa cancer cells. A standard scale of $10 \mu \mathrm{m}$ and 50 um for insets are deployed 
In general, molecules enter cells through passive diffusion, but nanomaterials enter cells predominately through endocytosis mechanism. A significant amount of research had already been conducted to determine and establish how nanoscale material enter cancer and fibroblast cells ${ }^{112-115}$. The theranosomes were found internalized near and in almost all cell components of both cancerous cells. Representative images of (primary and secondary) theranosomes interacting with both (HeLa and MDAMB-231) cancerous and NIH3T3 fibroblast cells is showcased in figure 3-5 wherein the primary theranosomes are visible in the (HeLa) nuclear membrane and in close proximity to (MDAMB-231) nucleus. A magnified Dark field TEM view of the theranosomes uptake is also presented in the figure 3-5A which are brightly illuminated with white spots and represented by red arrows. Interestingly, a significant portion of the primary theranosomes were located as clusters in the cellular structures, a phenomenon which is completely absent in fibroblast cells. But, the secondary theranosomes showed several clustered aggregations irrespective to cell phenotype from the TEM observations. Several research works have reported that both single and dispersed nanoparticles in general enter cells through endocytosis and tend to aggregate at cell membrane which results in receptor clustering ${ }^{112,116}$.

Numerous researches have also demonstrated a wide array of nanoparticle uptake mechanisms and pathways in cancerous and fibroblast cells to establish the therapeutic efficiency and also the drugtransport into cancer cells, specially Clathrin-mediated endocytosis (CME) mechanism at nano-bio interface level ${ }^{117,118}$. Since, CME plays a vital role in cellular process to transport nutrients, specific studies were focused on their interactions with cellular membrane involving unique set of interactions. For instance, Chithrani et al., demonstrated a detailed study detailing clathrin-dependent endocytosis mechanism of plasmonic gold nanoscale materials in breast cancer cells ${ }^{119}$. Similarly, Yallapu et al., showcased higher nanoparticle uptake of iron oxide by MDAMB-231 ${ }^{120}$. Endocytosis process dynamically modulates several cancer cell functions thereby responsible for its proliferation and migration. 


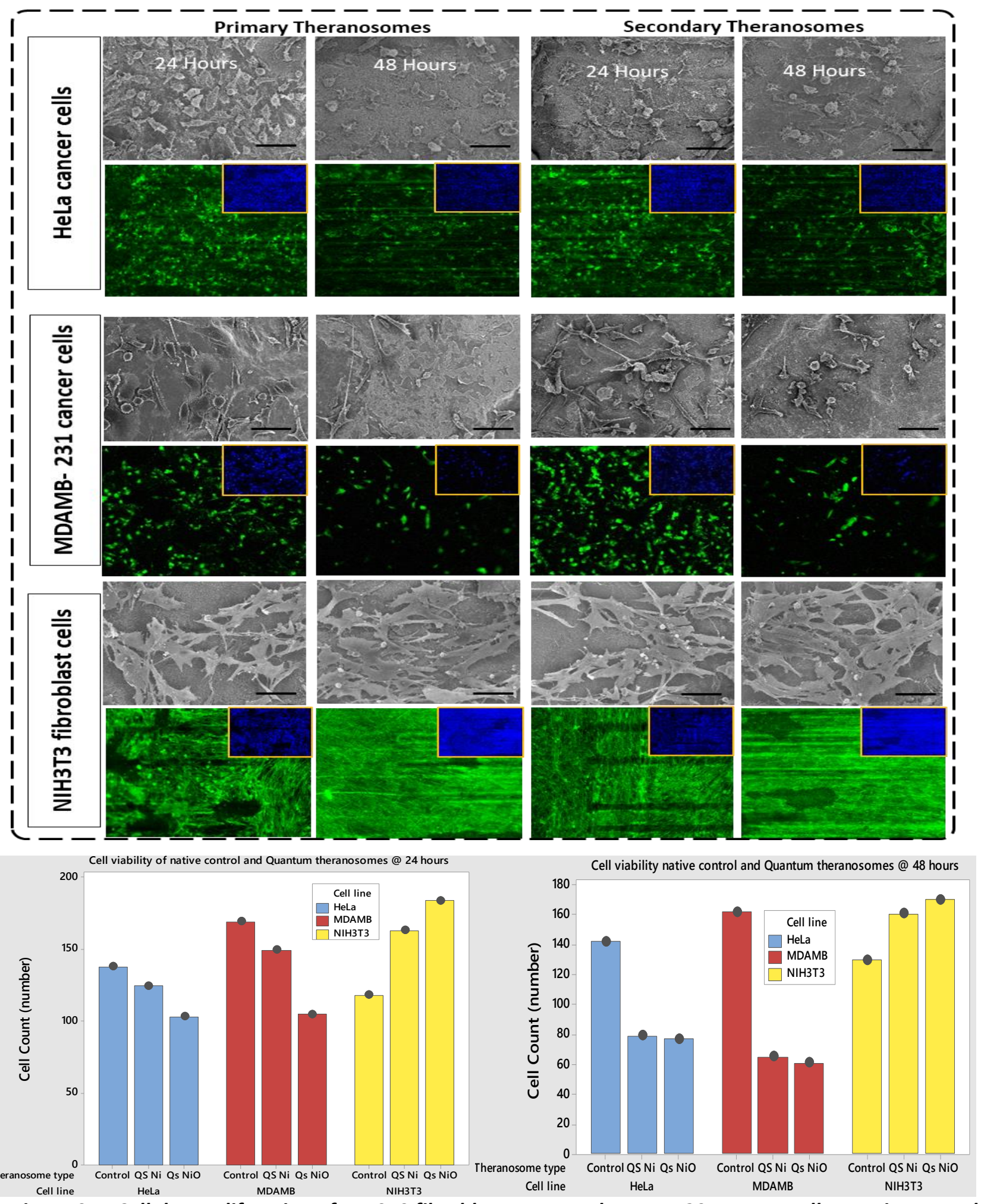

Figure 3-7. Cellular proliferation of NIH3T3 fibroblast, HeLa and MDAM-231 cancer cells on primary and secondary theranosomes. (a) Representative SEM \& fluorescence images showing the proliferation of three different cell lines, metastatic MDA-MB-231, and HeLa cancer cells, as well as the noncancerous NIH3T3 fibroblast cells 
Thus, it has been inferred that endocytosis in cancer cells is not only unregulated but also making the process quite different based on cell phenotypes when compared with normal cells ${ }^{117}$. Similarly, in this work it was found that the fluorescence intensity of NIH3T3 cells when compared with cancerous cells was significantly weaker on both primary (quantum-Ni) and secondary (quantum-NiO) theranosomes when compared with that of the corresponding native nickel control surface. In-addition, the results also indicated that the fluorescent intensity of both theranosomes was at its least on NIH3T3 fibroblast cells under all excitation channels when compared with that of the native control and (HeLa and MDAMB-231) cancer cell lines. This selective fluorescence excitement of theranosomes establishes the viability of using theranosomes for fluorescence bioimaging.

Furthermore, the primary (quantum-Ni) theranosomes probe showed increased fluorescence intensity when interacting with both (MDAMB-231 and HeLa) cancer cells when compared with corresponding fibroblasts and native control surface. Thus, primary theranosome probe acted as a turn ON fluorescence probe increasing fluorescence intensity when made to interact with cancer cells. This is validated by the data presented in figure 3-(5A and $6 A)$. The selective fluorescence excitation exhibited by the synthesized primary theranosomes can be explained using energy transfer reaction observed in plasmonic metallic materials such as gold and silver. These plasmonic materials due to increased accumulation and localization near cellular membrane introduce localized Surface Plasmon Resonance (LSPR) which in-turn brought out the Metal Enhanced Fluorescence (MEF) excitation. This reaction observed exhibits increased flourescence excitation upon all interacting cancerous cells when compared with NIH3T3 fibroblast cells ${ }^{121}$. This excitation reaction thus induced in cancer cells essentially acts as a light up bioprobe reaching increased fluorescence intensity through theranosome aggregation in cancer cells ${ }^{122,123}$.

However, the secondary theranosome exhibits high fluorescence intensity only on MDAMB-231 cells when compared with HeLa cells. This selective enhanced fluorescence intensity excitation exhibited by the secondary theranosome probe on MDAMB-231 cells is roughly twice when compared with its 


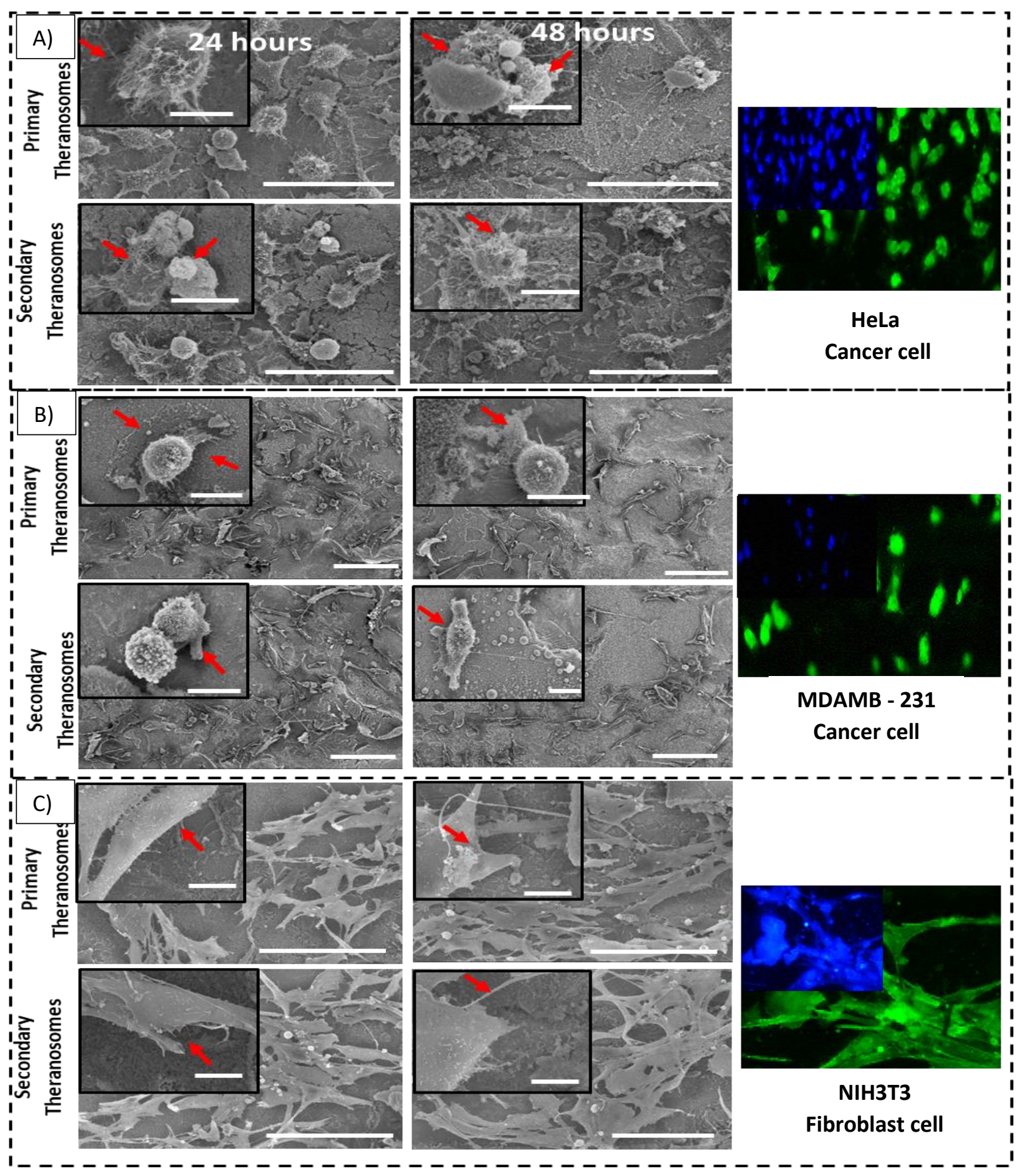

Figure 3-8: Influence of primary and secondary theranosomes on adhesion (24 hours) and proliferation phase (48 hours) A) Comparison between the quantum theranosomes over Hela cell adhesion B) Comparison between the quantum theranosomes over MDAMB cell adhesion. adhesion $C$ ) quantum theranosomes over NIH3T3 cell adhesion. A uniform scale bar of 10 and 3 um was followed. The red indicators are filopodial presence and their interaction on theranosomes 
interaction with HeLa cells. The enhanced fluorescence excitation of MDAMB- 231 cancer cells by secondary theranosome probe while simultaneous fluorescence quenching on HeLa cells establishes the abovementioned secondary theranosome probe as a viable cell specific fluorescence exciter. The secondary theranosomes being composed entirely of $\mathrm{NiO}$ acts as typical metal oxide particle. The presence of oxygen within the secondary theranosome supresses the spontaneous energy transfer reaction which is observed in primary theranosome through $M E F$, thus limiting their fluorescence excitation property and thereby requiring more energy per unit area to excite the fluorescence property $^{124}$. This mechanism essentially acted as a switch to turn ON and OFF the fluorescence property of primar and secondary theranosomes. This unique and specifici property of the quantum nickel oxide to excite only interacting MDAMB-231 cancer cells and not HeLa cells was an interesting aspect here as this specific property opens up the use of synthesized secondary theranosome (quantum-NiO) as an active cancer cell specific diagnostic agent. This effect was in congruent to the higher targeting efficiency exhibited by the secondary theranosome on MDAMB-231 cancer cells in inducing cell apoptosis when compared with HeLa cancer cells as can be seen from inset figure 3-7. Furthermore, the TEM images presented in figure $6 \mathrm{~B}$ also showcase less secondary theranosome accumulation but on the other hand more localization when compared with primary theranosomes.

\subsubsection{In-vitro therapeutic efficacy of theranosomes:}

NIH3T3 fibroblasts, MDAMB-231 and HeLa cancer cells are cultured both on native nickel and synthesized quantum theranosomes to investigate and evaluate their adhesion and proliferation response. The SEM results presented in the inset of figure 3-7 compares the HeLa, MDAMB-231 and NIH3T3 fibroblast cell population number and morphology response on native and synthesized quantum theranosomes. All the cells attached and spread over well evenly within the 24 hours duration without the need for any special treatment with ECM molecules prior to cell incubation making the synthesized quantum theranosomes 


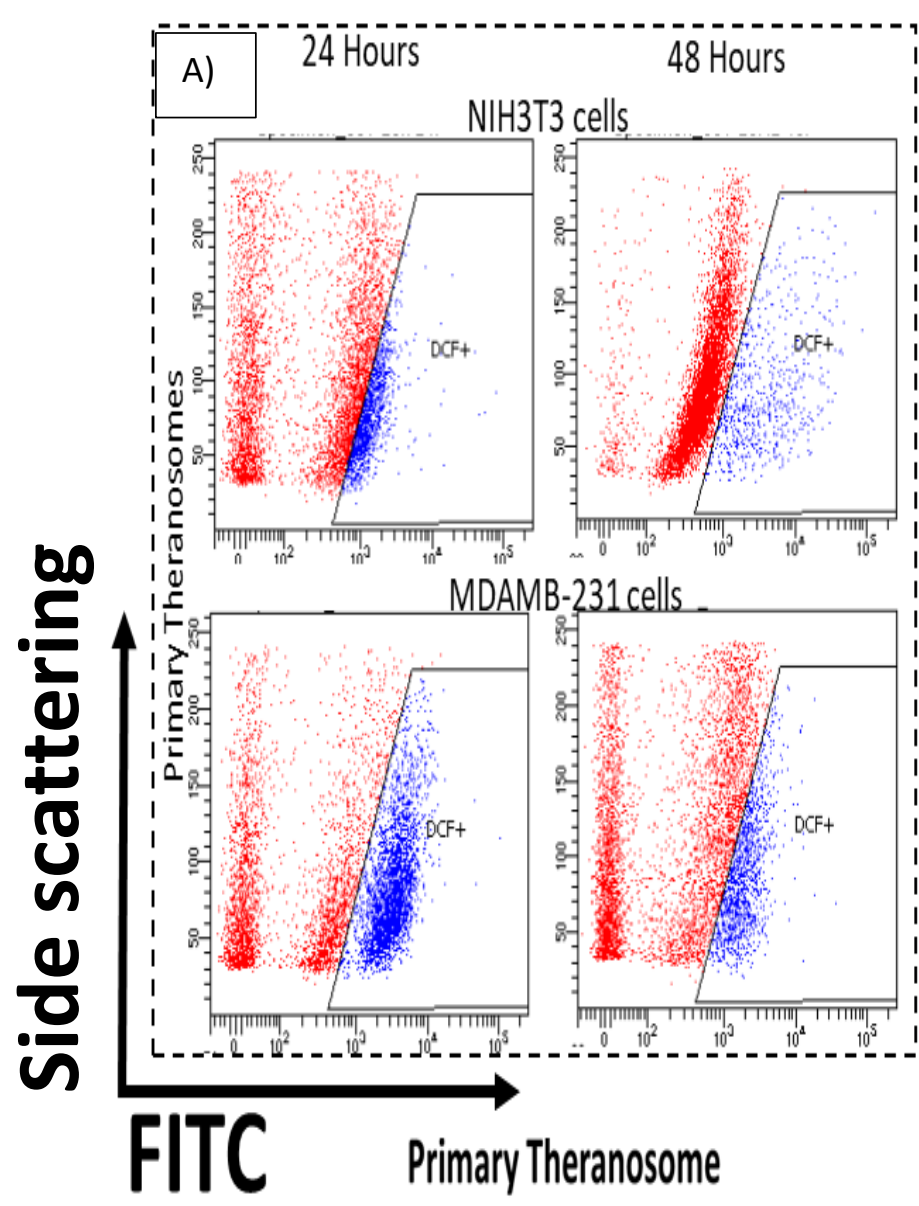

Reactive Oxygen Species (ROS) generation

C) 60

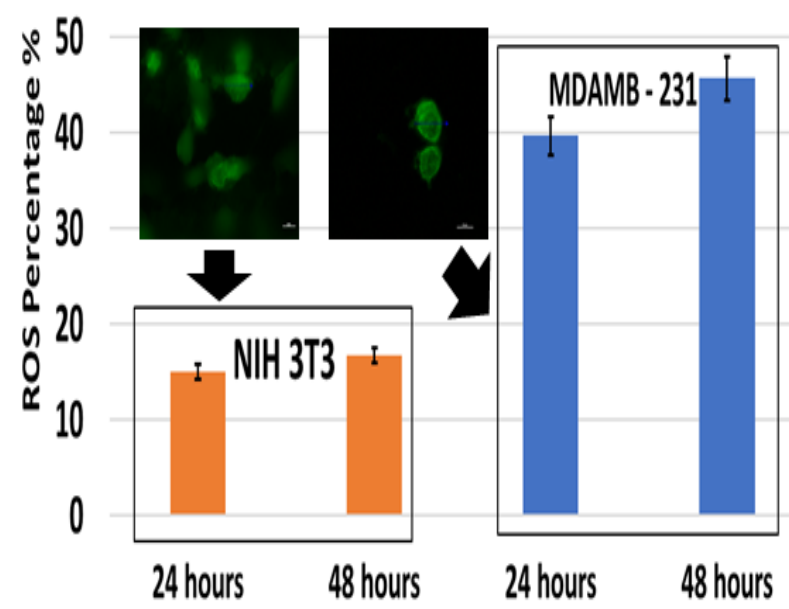

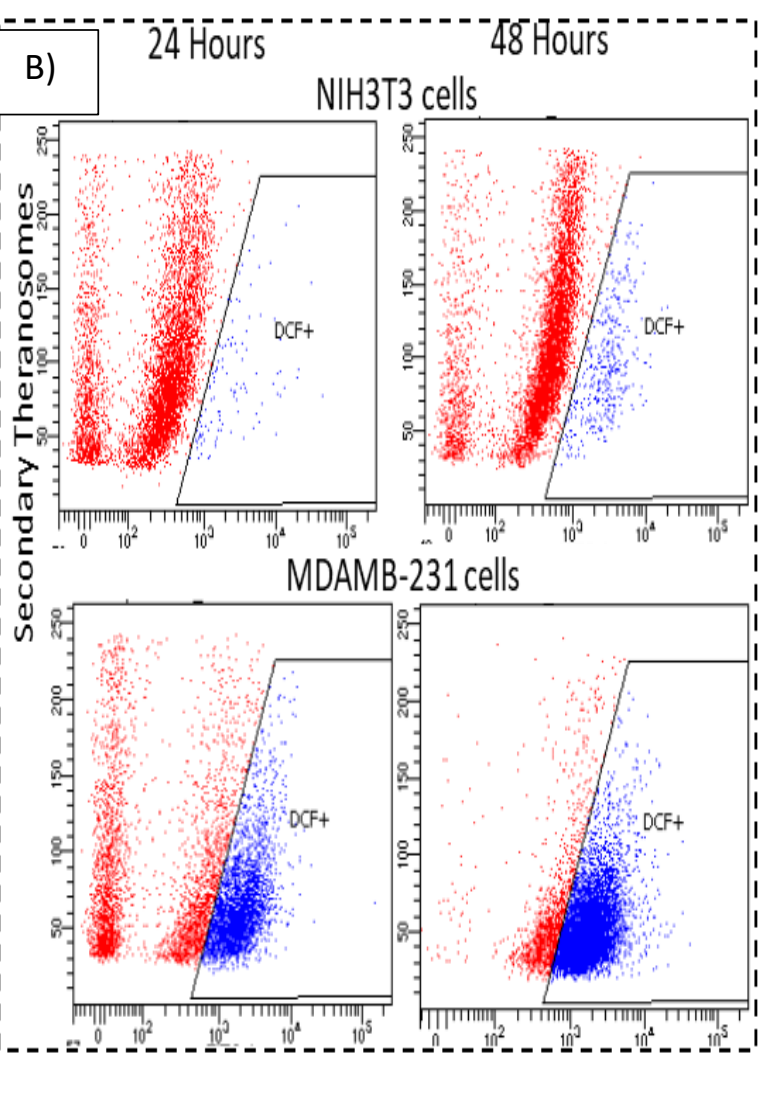

Secondary Theranosome

Reactive Oxygen Species (ROS) generation

100

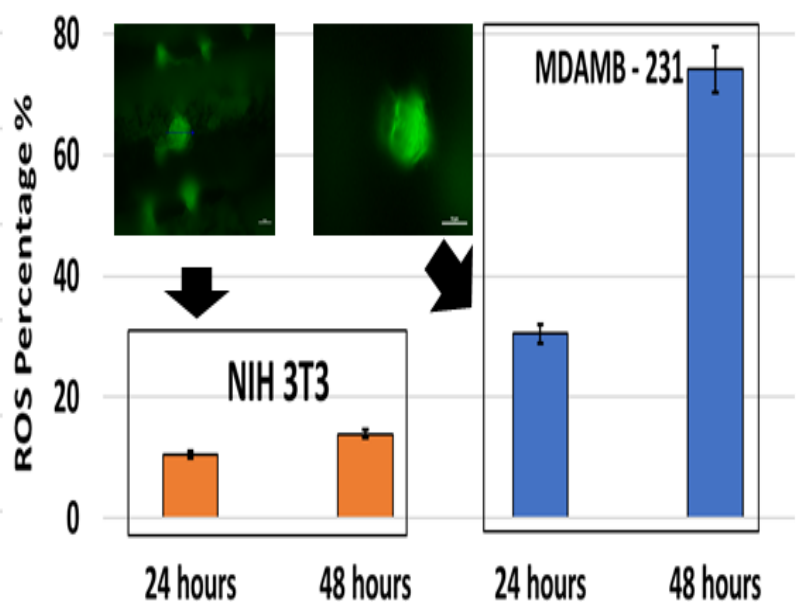

Figure 3-9: ROS generation- DCFH-DA assay. The dot plots in blue color indicate the cells under ROS through increased fluorescence intensity with respect to control. A) NIH3T3 and MDAMB-231 cells treated with primary $B$ ) secondary theranosomes (C) graph showing the percentage of cells in showing $D C F$ fluorescence. The data represents mean $\pm S D$ of three independent experiments 
act and mimic ECM like behavior irrespective of the cell type. The NIH3T3, MDAMB-231 and HeLa cells proliferated on native nickel were observed to be smaller in size and were randomly distributed throughout the substrate. However, the number, morphology and organization of cancerous (HeLa \& MDAMB-231) and NIH3T3 cell adhesion on quantum theranosomes were different from that of the cells that were attached to the native nickel substrates. In addition, the interacting cells systematically proliferated and grew in an organized manner with stretched filopodia and lamellipodia as can be seen from inset figure 8 . The size of cells adhered to quantum theranosomes were larger in size compared to the cells proliferated upon the native nickel substrates. The NIH3T3 fibroblast cells present on quantum theranosomes were closely self-organized and almost covered by one monolayer thickness making it hard to distinguish one cell from another.

A pronounced self-organized cell coverage was observed during the first 24-hour incubation on both primary and secondary theranosomes. This trend continued even with a prolonged incubation time of 48 hours on both the native nickel and theranosomes as can be seen in the inset figure 7 and 8 . The quantum theranosomes however altered fibroblast cell behaviour after 48 hours duration with more evidence of elongated cell morphology present on quantum theranosomes suggesting cell migration had taken place. Nonetheless, the in-vitro response of MDAMB-231 and HeLa cells on the quantum theranosomes revealed remarkable cell governing properties.

At first, we investigated the influence of MDAMB-231 and HeLa cells on both the 3D quantum theranosomes which played a key role in controlling the cancer cell behaviour when compared with fibroblast cells as seen from the figure 7. They demonstrated an increased fibroblast cell adhesion when compared with the cancer cells. The statistical results of the same after 24-hour incubation are presented in the inset show that the total cancer cell count reduced significantly on both quantum theranosomes when compared with native nickel control samples from 24 to 48 hours. 


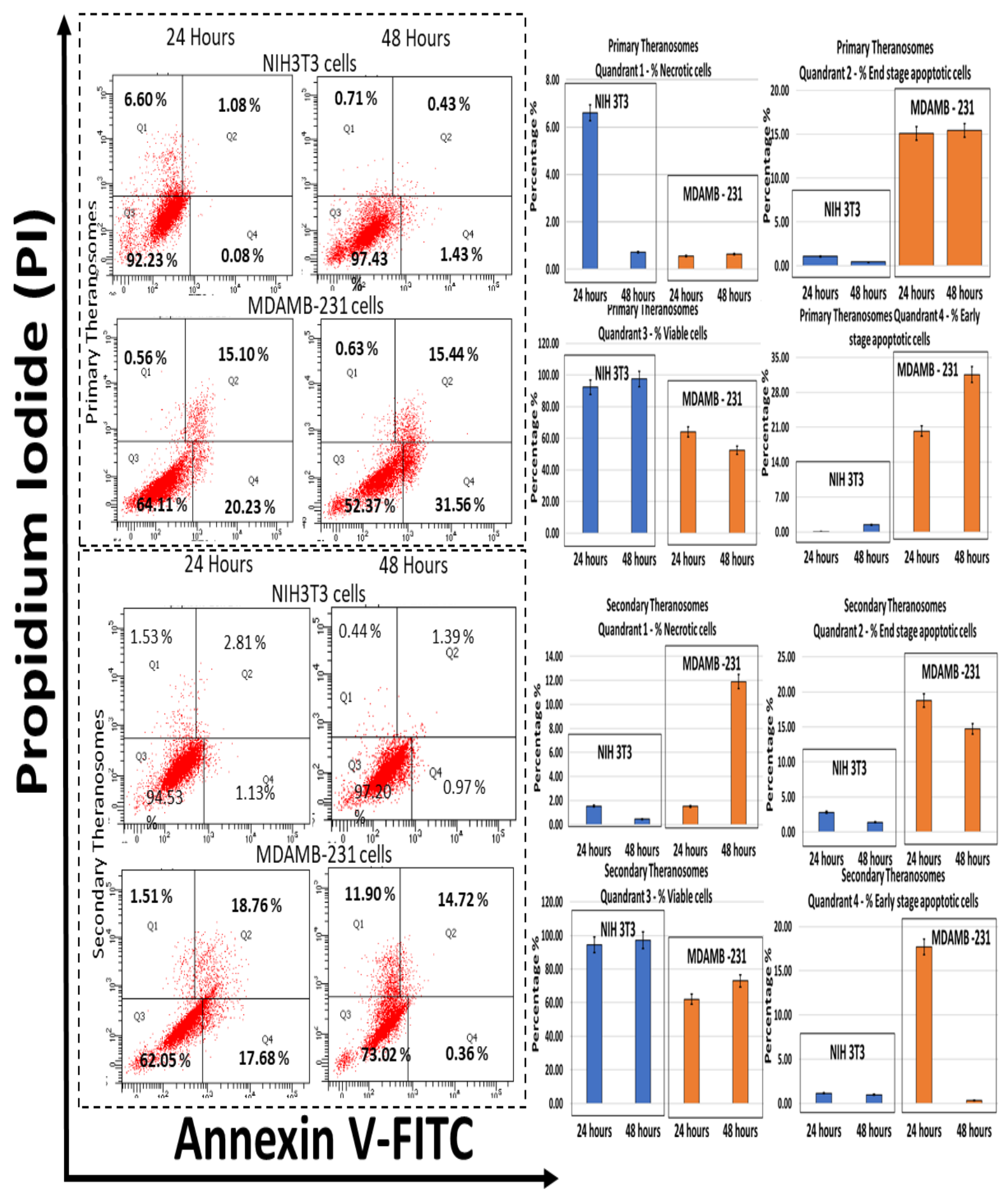

Figure 3-10: Cell apoptosis determined by Annexin V-fluorescein isothiocyanate/ propidium iodide staining. The results were determined after incubation with primary and secondary theranosomes in MDAMB cancer cells and NIH3T3 fibroblast cells for 24 and 48 hours incubation ( $n=3)$. A standard minimum of 10000 events were recorded for each plot. 
A complete reversal of the cell attachment was noted with respect to the particle size wherein, quantum web acted as a decoy by turning off its proliferative behaviour during the 48-hour incubation period. We can certainly establish a grade of cancer killing efficiency of an increasing order based on the observed results with secondary and primary theranosomes having low and high degree of killing efficiency. The fluorescence microscopy analysis also suggests the same trend as can be seen from inset figure 3(7 and 8). On the other hand, the fibroblast cell adhesiveness on quantum theranosomes does not seem to alter even with an increased incubation time of 48 -hour. This infers that by varying the multiphoton ionization energy, both the material chemistry and physical architecture of the synthesized theranosomes. This unique and specific property of the quantum nickel oxide to excite only interacting It is observed from the results presented herein that NIH3T3 cells is less susceptible to theranosomes when compared to MDAMB-231 and HeLa cells. Earlier studies on tumor microenvironment have demonstrated its vital role in regulating cell adhesion, sensing and signalling aspects. Emerging works in 3D tumor environment have now pointed out that the current need for incorporating ECM proteins and specific biomolecules are both to alter the cell morphologies, adhesion, proliferation and migration properties ${ }^{125}$. The 3D porous structures due to its heterogeneous nature have shown attributable traits in cellular adhesion and migration when compared with its one and two-dimensional counterparts. Unlike the conventional anticancer agents that straight away attack cancer cells right from the beginning, this quantum theranosomes are acting more as a targeted agent. It presented favourable cues to cancer cells initially, thereby promoting adhesion and proliferation. But, apoptosis was then induced after a prolonged incubation time of 48-hour through autophagy. Moreover, this apoptosis was induced solely by the particles present in the theranosomes architecture, making it a quantum medicine rather than a mere drug carrier. Distinct biochemical and physical changes involving cell cytoplasm, nucleus and plasma membrane are often associated with apoptotic cell death ${ }^{15}$. In a tumor microenvironment, during an early stage of apoptosis, predominately cells cluster to round up thereby, losing contact with its surrounding cells and then 
subsequently start to shrink. The filopodia of interacting HeLa, MDAMB-231 and NIH3T3 cells observed via SEM can be seen from inset figure 3-8 (A, B \& C) was employed to study the adhesion and proliferation properties of cells upon synthesized theranosomes. The filopodial development on the native nickel was quite random in nature. On the contrary, cells interacting on quantum theranosomes showed a drastic change in the number of filopodia during the first 24-hour incubation period. The cells that proliferated had on an average less filopodia with an altered morphology when compared with the basic native nickel substrate. It is a well-known aspect that the cells need to spread so as to stratify the various phases of cell growth. Thus, filopodia that adhere to substrates typically originated from random locations within the cell body and at a certain distance above the surface. These filopodia thus come under contact with the underlying substrate under a broad range of angles as can be seen from inset figure 3-8A. It could also be seen that apart from the filopodia interacting with the theranosomes structure, so were the retraction fibers of the cell as can be seen from inset figure 3-8A. The presence of filopodia and retraction fibers at the end points demonstrate the presence of high degree incidence of cellular and synthesized quantum theranosomes interaction having already taken place.

\subsubsection{Intracellular Reactive Oxygen Species (ROS) assessment of Quantum Theranosomes:}

Reactive oxygen species (ROS) plays a critical role in the early stages of cell apoptosis. Mitochondria was considered both as a source and also as a target of ROS exhibited by these cells. In general, when excess ROS was generated, it persuades Mitochondria Membrane Potential (MMP) depolarization to release cytochrome c, which stimulates caspase activation. The ROS generation can also induce DNA damage, which then promotes apoptosis ${ }^{126}$. Wang et al. demonstrated that Nickel refining fumes induced DNA damage and apoptosis on NIH3T3 fibroblast cells through oxidative stress ${ }^{127}$. In addition, Pan et al., showed Nickel sub sulfide (Ni3S2) induced ROS-mediated apoptosis in human bronchial epithelial BEAS2B cells through p38 signalling pathway ${ }^{128}$. Furthermore, Ahamed et al., suggested that nickel nanoparticles induce oxidative damage, which decreases glutathione (GSH), and induces ROS and lipid 
peroxidation (LPO) in human lung epithelial A549 cells. Hence excess generation of ROS will eventually result in oxidative stress that mediates apoptosis ${ }^{52}$. Although several previous studies had shown induction of ROS induced cell death in various cancer and fibroblast cells by different nanoparticles, they were all limited to colloidal nanoparticle suspensions whose dimensions were greater than $20 \mathrm{~nm}$. Hence, we were quite interested to investigate the effect of 3D quantum theranosomes synthesized here focussed more towards ROS generation in MDAMB-231cancer cells and NIH3T3 fibroblast cells. For this study, MDAMB-231 and NIH3T3 cells were loaded with the ROS measuring probe $2^{\prime}, 7^{\prime}$-dichlorodihydro fluorescein diacetate ( $\left.\mathrm{H}_{2} \mathrm{DCFDA}\right)$ and studied using flow cytometry. As shown in the figure 9 , both primary and secondary quantum theranosomes induced ROS generation in a time dependent manner, as indicated by an increase noted in fluorescence intensity. The ROS generated was at its peak after 48 hours of exposure to both quantum theranosomes in MDAMB-231 when compared over untreated MDAMB-231 controls as can be seen from Appendix B figure S3 respectively. The primary (quantum-Ni) theranosomes observed an increased ROS generation (39.69\%) after 24 hours incubation period, when compared with secondary (quantum-NiO) theranosomes (30.5\%). However, with an increased incubation period of 48 hours the ROS intensity increased significantly for primary theranosomes (45.68\%) when compared with secondary theranosomes (74.11\%) depicting a cell selective physiochemical dependent ROS mediated apoptosis upon MDAMB-231 cancer cells. Thus, a substantial increase in MDAMB-231 cells ROS was observed when compared between the primary and secondary theranosomes. Concurrently, the same quantum theranosomes induced a very negligible ROS generation to NIH3T3 cells. A representative figure showing the difference in the fluorescence intensity is highlighted in the figure 3-9 with normal proliferating cells showing decreased level of fluorescence when compared with an apoptotic cell. 


\subsubsection{Cell death induction by 3D quantum theranosomes and assessment of Necrosis and}

apoptosis:

Since the production of ROS is a major mediator for the activation of apoptosis signalling pathways, the influence of 3D quantum theranosomes on cancer and fibroblast cell apoptosis was evaluated by the annexin V -FITC/PI conjugate staining assay at 24 and 48 hours incubation period. The results obtained are presented in figure 10 and in Appendix B figure S3 along with respective statistical analysis. Quadrant 3 highlighted in the graphs in figure 3-10 represent the percentage of viable cells present during the analysis. Both the quantum theranosomes presented an increased percentage of NIH3T3 fibroblast cells when compared with MDAMB-231 cancer cells irrespective of the incubation period. The percentage of cells in quadrant 4 indicated an early stage apoptotic cell death suggesting that the cells present in that quadrant are beyond viability. The primary theranosomes presented a significant percentage increase in early stage cancer cell apoptosis when compared with fibroblast cells with increased incubation time from 24 to 48 hours. On the contrary, the secondary theranosomes presented an increased percentage of early apoptosis in cancer cells only during the first 24 hours time period. Additionally, the percentage of cells in quadrant 2 highlighted the percentage of end stage apoptotic cell event in both NIH3T3 and MDAMB-231 cells. Both the theranosomes recorded a lower level, less than $5 \%$ events ( 24 and 48 hours) of end stage fibroblast cell apoptosis but offered an increased end stage apoptosis in MDAMB-231 cells. Furthermore, the percentage of cells stained positive for Proprium lodide (PI) was observed from quadrant 1 indicating a loss of membrane integrity which was a clear indication of necrotic cell death. The quantum theranosomes irrespective of the incubation time induced cell selective apoptosis and necrosis to MDAMB cancer cells only. But, the NIH3T3 cells showed some signs of necrosis on primary quantum theranosomes (6\%) during the first 24 hours only and however after an increased incubation time showed a reversal of trend $(<1 \%)$ wherein no signs of both apoptotic and necrotic cells were observed. The secondary theranosomes results for NIH3T3 cells also followed an identical trend over the 24 and 48 hours time 
period. But, the secondary theranosomes showcased almost 600\% percent change in MDAMB-231 cancer cell necrosis between the 24 to 48 -hour incubation period. The above result very clearly demonstrated the cell selective apoptotic behaviour exhibited that was induced only by these specifically engineered and fabricated 3D quantum theranosomes.

\subsection{CONCLUSION:}

Theranosomes hold great promise for the future in the field of cancer treatment as they can detect (imaging), diagnose cancer and thereafter induce cancer apoptosis (theranostics) in a single step process. The magnetic quantum theranosomes herein presented propose a new direction for cancer theranostics using label-free broadband fluorescence excitation and cancer therapeutics. The synthesized (primary and secondary) theranosomes provided both magnetic and optical response allowing to conduct both labelfree fluorescent imaging and cancer therapeutic functions through quantum confinement. The proposed primary (Quantum-Ni) theranosome identified cancer (HeLa and MDAMB-231) cells from NIH3T3 fibroblast cells by enhancing the cancer cell fluorescence excitation which in a way is a diagnostic process. The secondary (Quantum-NiO) theranosome distinguished the MDAMB-231 cells from those of the HeLa cells through its specific fluorescence excitation thereby acting as screening agents by selectively enhancing only the fluorescence signals of MDAMB-231 cancer cells through fluorescence imaging. Inaddition, the in-vitro study conducted confirmed and demonstrated the biocompatibility of the proposed quantum theranosomes wherein the 3D architecture empowered mammalian cell exhibited selective adhesion and proliferation but later selectively inducing (HeLa and MDAMB-231) cancer cell apoptosis mimicking tumor microenvironment. Although favourable biocompatibility is attributed to quantum theranosomes, the intracellular oxidative stress derived through reactive oxygen species and the apoptotic pathway assessment clearly demonstrated the innate therapeutic functionality exhibited by these synthesized theranosomes when compared with those of the fibroblast cells. The theranosomes 
presented in this research is synthesized using a novel one step multiphoton ionization mechanism arranged in 3D fashion. The evidence recorded and presented in this research demonstrate the suitability of magnetic quantum theranosomes as a potential candidate for future cancer theranostics. This simplified approach will pave way for the next generation cancer theranostics which should be of interest to a broad spectrum of researchers in the field of theranostics. 


\section{CHAPTER 4}

\section{SERS ACTIVE NANOBIOSENSOR FUNCTIONALIZED BY SELF-ASSEMBLED 3D NICKEL NANONETWORKS FOR GLUTATHIONE DETECTION}

Published in ACS Applied Materials. Interfaces 2017,

We introduce a "non-noble metal" based SERS active nano-biosensor using a self-assembled 3D hybrid nickel nanonetwork. A tunable biomolecule detector fabricated by a bottom-up approach was functionalized using a multiphoton ionization energy mechanism to create a self-assembled 3D hybrid nickel nanonetwork. The nanonetwork was tested for SERS detection of crystal violet (CV) and glutathione (GSH) at two excitation wavelengths, $532 \mathrm{~nm}$ and $785 \mathrm{~nm}$. The results reveal indiscernible peaks with a limit of detection (LOD) of 1 picomolar (pM) concentration. An enhancement factor (EF) of $9.3 \times 10^{8}$ was achieved for the chemical molecule $\mathrm{CV}$ and $1.8 \times 10^{9}$ for the biomolecule GSH, which are the highest reported values so far. The two results, one being the CV molecule proved that nickel nanonetwork is indeed SERS active and the second being the GSH biomolecule detection at both $532 \mathrm{~nm}$ and $785 \mathrm{~nm}$, confirm that the nanonetwork is a biosensor which has potential for both in-vivo and in-vitro sensing. In addition, the selectivity and versatility of this biosensor is examined with biomolecules such as L-Cysteine, L-Methionine and sensing GSH in cell culture medium which mimics the complex biological environment. The functionalized self-assembled 3D hybrid nickel nanonetwork exhibits electromagnetic and charge transfer based SERS activation mechanisms.

\subsection{INTRODUCTION:}

The development of functionalized nanomaterials has attracted considerable interest in the field of diagnostics and bio sensing because nanometer-scale materials offer several properties that bulk materials lack ${ }^{129,130}$. A significant challenge in bioanalytical sensing is the development of highly sensitive 
platforms that overcome the limitations of conventional sensing techniques, which include electrochemical ${ }^{131-133}$, piezo-electric ${ }^{134}$, and thermometric sensing ${ }^{135}$. With the increasing usage of gold nanoparticles, optical sensing techniques, such as UV-Vis spectroscopy, resonance elastic light scattering, fiber optics sensing, and spectrofluorometric sensing, have gained much attention in biomedical science because of their increased sensitivity, improved spatial resolution, and throughput capability ${ }^{18-20}$. Among the various optical sensing mechanisms, surface enhanced Raman scattering (SERS) is most valuable owing to its non-invasive nature, higher sensitivity, and broad range of excitation wavelengths with various nanomaterials. SERS enhancement offers unique spectral molecular fingerprint data from the interaction area with limited photo bleaching, reduced background interference, auto fluorescence, and high sensitivity, as compared with other optical detection techniques such as fluorescence and electrochemical sensing ${ }^{136}$. Numerous semiconductor-based nanomaterials, such as $\mathrm{TiO}_{2}, \mathrm{ZnO}$, graphene, and CuTe, have been reported to be SERS platforms, but their enhancements $\left(\sim 10^{2}-10^{4}\right)$ are relatively weak compared to enhancements provided by SERS platforms based on noble transition metals $\left(\sim 10^{4}-10^{8}\right)^{61,131,137}$. Noblemetal monolayer-coated semiconductor hybrid nanostructures with varying morphologies have also been used as SERS substrates for multi-analyte detection ${ }^{138-140}$. Noble-metal-based nanomaterials have demonstrated superior properties in terms of increased SERS sensitivity by the tuning of their optical absorption properties, band gap, and excitation Bohr radius ${ }^{141,142}$. This has enabled detection at very low concentrations of analytes and has even led to the possibility of single molecule detection with SERS enhancement factors ranging from $\sim 10^{6}$ to $10^{12}$ by using noble-metal-based nanomaterials ${ }^{143-145}$. Therefore, most current research is devoted to developing either noble metals or noble-metal-coated semiconductor nanomaterials as SERS active substrates.

Noble metals exhibit localized surface plasmon resonance (LSPR) when excited by photons. Apart from SPR, charge transfer resonance and hotspots are also hypothesized to contribute towards SERS enhancement in both semiconductor and metal-based nanostructures ${ }^{141,142}$. Investigations have also 
revealed that nanostructure morphology plays a pivotal role in inducing SERS exertion. Noble metal nanostructures, including nanostars, nanofilms, nanocarpets, nanorods and hollow or solid nanocubes, have been synthesized for investigation in this direction ${ }^{146-149}$. However, noble metal and semiconductornoble-metal hybrid nanomaterials have several limitations making them undesirable for a variety of biomedical applications. Noble-metal-based substrates are often synthesized using chemical reduction, oxidation, or physical deposition processes, which lead to poor biocompatibility and thereby severely limit their use as biomolecular sensing materials ${ }^{150}$. As a result, the need for "non-noble metal" based SERS active nanostructured material with strong biocompatibility is of utmost importance for bio-sensing applications. In this regard, nickel nanomaterials have received considerable attention. Being a prime magnetic transition material with excellent biocompatibility, nanoscale nickel is extensively used as contrast agents for MRI diagnosis, hyperthermia, and cancer therapeutic applications ${ }^{42,151}$. However, results reveal that nickel nanowires synthesized by electrochemical process are Raman insensitive because of the inherent characteristics of nickel. Interestingly, gold coated nickel nanowires fabricated by the same method are noted to be Raman active ${ }^{152}$.

Some studies have prepared nickel-based nanostructures for SERS with conventional fabrication techniques, including galvanic displacement, redox transmetalation, and electrochemical synthesis. Sanjanlal et al. used the galvanic displacement technique to fabricate nickel nanowires and nanocarpets coated with noble metals ( $\mathrm{Au}, \mathrm{Ag}$ ) for facilitating SERS sensing of trace analytes ${ }^{153}$. Similarly, Manoj et al. demonstrated the Raman detection of methylene blue analyte by using composite $\mathrm{Ni} / \mathrm{Ag}$ nano flowers fabricated by redox transmetalation ${ }^{154}$. By adopting an electrochemical technique, Bayata et al. reported the fabrication of a honeycomb template with nickel nanowires self-assembled inside anodic aluminum oxide. A thin layer of gold was deposited on the honeycomb. In these experiments, the SERS was found to be enhanced by the gold nanoparticles. The nickel nanostructures acted as a carrier of the gold nanoparticle and a platform to attract and adhere biomolecules, and they in no way assisted in actively 
inducing Raman scattering. Thus, these highly anisotropic nanomaterials fabricated using solution-based methods are dependent on the use of noble metal coatings such gold and silver to accomplish SERS enhancement at 532 and $785 \mathrm{~nm}^{152}$. Hence, there is a significant incentive to fill the need of SERS active nickel-based nanostructures, which will further the development of optical-based detection nanobiosensor platforms and reduce the reliance on noble metals as SERS activators.

In this research, we developed a nano-biosensor using a functionalized hybrid nickel nanonetwork for biomolecule sensing and detection. The self-assembled 3D hybrid nickel nanonetwork is functionalized by multiphoton ionization induced by a high-energy femtosecond laser. The nanonetwork can activate Raman detection of crystal violet $(\mathrm{CV})$ and glutathione $(\mathrm{GSH})$ molecules at $1 \mathrm{pM}\left(\sim 1 \times 10^{-12} \mathrm{M}\right)$ concentrations at two excitation wavelengths, $532 \mathrm{~nm}$ and $785 \mathrm{~nm}$. An enhancement factor of $10^{9}$, a level not observed before even with noble metal monolayer coatings, is seen here. This extends the limit of detection (LOD), making the self-assembled 3D hybrid nickel nanonetwork a promising "non-noble metal" based candidate for chemical and biomolecular sensing. The 3D hybrid nickel nanonetwork proposed here could open up new vistas in the field of nano-biosensors. 


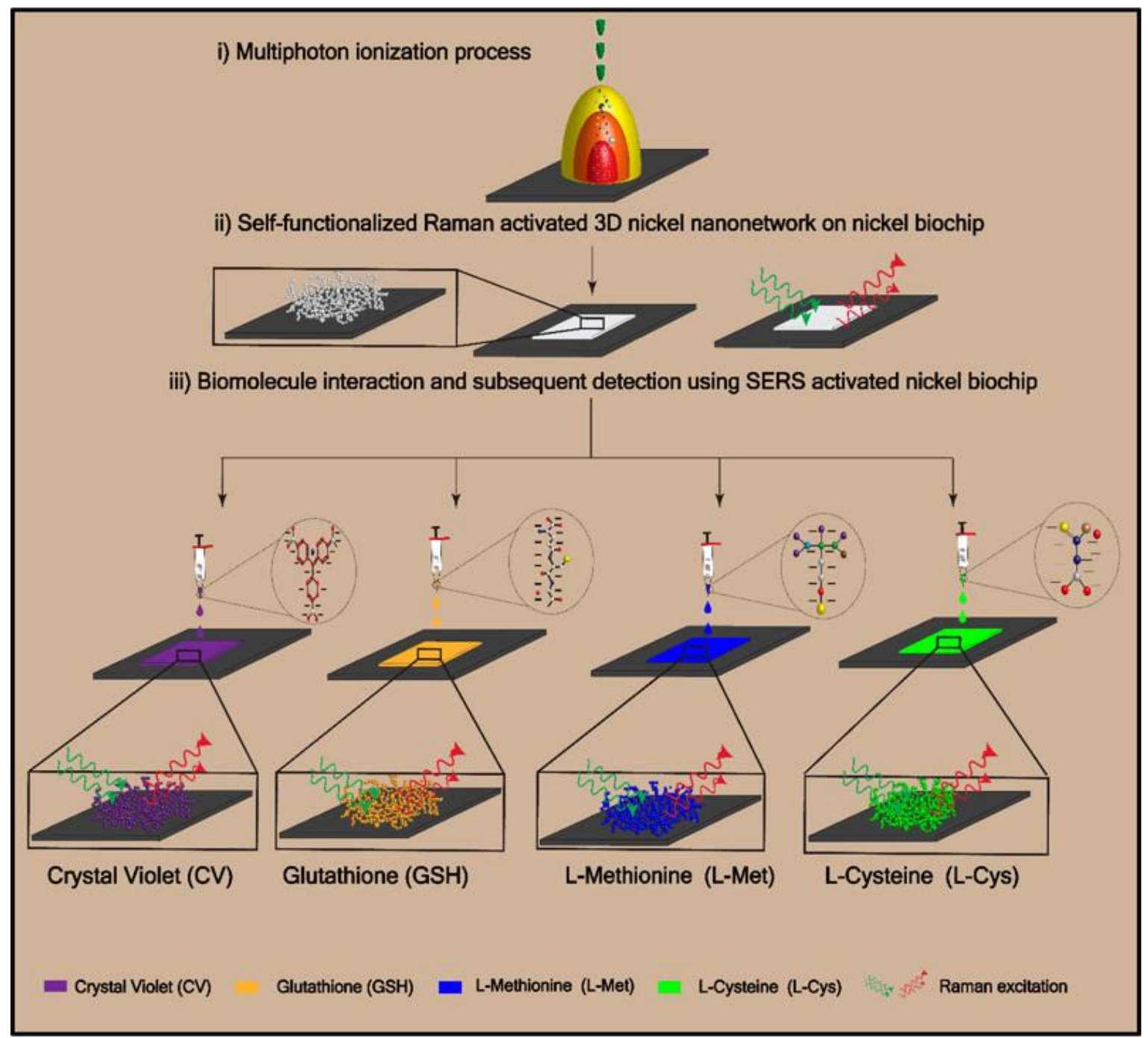

Figure 4-0: Overall schematics of SERS-active nanobiosensor synthesis and subsquent biomolecule interaction

\subsection{Materials ANd Methods:}

\subsubsection{Materials:}

Commercially available Nickel 200 sheets ( $3 \mathrm{~mm}$ thickness) having $99 \%$ nickel were used in all experiments. The surface was subjected to gradual mechanical polishing using SiC grit sandpaper (240600 ) and later ultra-sonically cleaned in $100 \%$ acetone and ethanol for a duration of 15 min each to remove all embedded particle inclusions during polishing. 


\subsubsection{Preparation of Crystal Violet Substrate:}

Crystal violet dye was selected because of its large Raman cross-section, and it is used often for testing Raman scattering. A stock solution of CV was prepared from anhydrous powder purchased from Sigma Aldrich to an initial concentration of $1 \mathrm{M}$ and sequentially diluted to $1 \times 10^{-3} \mathrm{M}, 1 \times 10^{-6} \mathrm{M}, 1 \times 10^{-9} \mathrm{M}$, and $1 \times 10^{-12} \mathrm{M}$ with ultra-pure water for experimentation.

\subsubsection{Preparation of Glutathione Substrate:}

L-Glutathione reduced powder was purchased from Alfa Aesar, and a stock solution was prepared using ultrapure water to $1 \mathrm{M}$ concentration. A series of dilutions were made later using ultra-pure water to $1 \times$ $10^{-3} \mathrm{M}, 1 \times 10^{-6} \mathrm{M}, 1 \times 10^{-9} \mathrm{M}$, and $1 \times 10^{-12} \mathrm{M}$ concentrations and kept in a refrigerator maintained at $4^{\circ} \mathrm{C}$ covered with metal foil to protect it from direct light.

\subsubsection{Preparation of L- Cysteine Substrate:}

L-Cysteine hydrochloride powder was purchased from Sigma Aldrich and a series dilution of $1 \mathrm{mM}$ and $1 \mu \mathrm{M}$ concentration was prepared with ultrapure water for experimentation.

\subsubsection{Preparation of L-Methionine Substrate:}

L-Methionine powder was purchased from Alfa Aesar and a series dilution of $1 \mathrm{mM}$ and $1 \mu \mathrm{M}$ concentration was prepared with ultrapure water for experimentation.

\subsubsection{Preparation of complex biological substrate:}

Biological cell culture medium DMEM-F12 medium was purchased from Gibco Life technologies.

\subsubsection{Preparation of GSH with complex biological substrate:}

The prepared L-Glutathione stock solution is diluted to $1 \times 10^{-6} \mathrm{M}$ concentration using ultrapure water and further diluted to prepare $1 \mu \mathrm{M}$ concentration of GSH solution. A $10 \mu \mathrm{L}$ of this solution is then added to $1 \mathrm{ml}$ biological cell culture DMEM-F12 medium to prepare a complex biological GSH solution. 


\subsubsection{Multiphoton lonization Procedure:}

A diode-pumped Yb-doped fiber laser from Impulse series (Clark - MXR) having a central wavelength of $1030 \mathrm{~nm}$ and an average power of $16 \mathrm{~W}$ was employed for fabricating the self-assembled 3D hybrid nickel nanonetwork. To do this, an ultra-short pulsed laser (UPL) system having a varying laser pulse interaction time of $214 \mathrm{fs}$ to $1428 \mathrm{fs}$ with a laser pulse repetition rate between 4 and $25 \mathrm{MHz}$ was made to interact with the prepared nickel samples. The samples were mounted on $x-y-z$ precision stages to steer the incoming laser beam. To maximize the effectiveness of the fabrication, some of the processing parameters were kept constant. The laser pulse interaction time was fixed at $214 \mathrm{fs}$, average power at 16 watts, and dwell time at $5 \mathrm{~ms}$, which is the time spent delivering laser the pulses to a single point on the specified sample. Based on earlier studies using Clark-MXR Impulse series laser, a laser spot size of $1.02 \mu \mathrm{m}$ was used for the creation on the 3D nanostructure. An array of ablation points was created on the target sample to obtain a workable quantity of 3D nanostructures. EzCAD software and a galvoscanner were deployed for the plotting. The experimental setup and fabrication procedure are presented in the inset of Figure 4-1. The ionization energy was varied in order to obtain the self-assembled 3D nanonetwork. The experimental parameters employed are listed in Table B S1 along with their labels: Cuboidal, SpheroidalCuboidal, and Spheroidal nanonetworks.

\subsubsection{Scanning Electron Microscope:}

The fabricated nickel specimens were evaluated initially using an environmental scanning electron microscope (ESEM; Quanta FEG 250). To evaluate and study the effects induced by the multiphoton ionization energy on the fabricated 3D nanonetwork, a high-resolution scanning electron microscope (HRSEM; Hitachi S5200) was employed. A carbon copper grid was used to collect the nanonetwork, and demangetized using a Neodymium magnet before examination. Then, an HRSEM \EDX attachment from Oxford Instruments was used to primarily analyze the elemental composition of the synthesized nanonetwork. 


\subsubsection{AFM Analysis:}

To examine the oxide phases and morphological variations in the synthesized self-assembled 3D nanonetwork, an atomic force microscope (AFM; ND-MDT, Russia) was used. A prefabricated gold-coated silicon cantilever tip with a spring constant of $15 \mathrm{~N} / \mathrm{m}$ is employed in both the contact and non-contact mode at a scan rate of $0.05 \mathrm{~Hz}$ each to acquire images at a scan area of $100 \times 100 \mu \mathrm{m}$.

\subsubsection{X-Ray Diffraction:}

To quantitatively and qualitatively identify the oxide phase concentration, an X-ray diffraction (Phillips powder XRD) was employed with Cu K- $\alpha$ radiation $(\lambda=1.54054 \AA$ ) at $40 \mathrm{KV}$ and $40 \mathrm{~mA}$ with a $2 \vartheta$ scanning speed of $0.025^{\circ} \mathrm{min}^{-1}$ scanning a range from $30^{\circ}-80^{\circ}$. Rietveldt analysis was carried on the acquired spectra, and crystallite size measurements of the nickel and nickel oxide nanoparticles formed within the 3D nanonetwork were estimated using the Scherrer equation.

\subsubsection{Raman Spectroscopy:}

The synthesized specimens were analyzed using a Bruker Senterra Dispersive Raman Microscope fitted with a multi-wavelength laser system (532 nm and $785 \mathrm{~nm}$ ). An appropriate laser irradiation power of 5 and $10 \mathrm{~mW}$ is used with a standard collection time of $10 \mathrm{~s}$ with three repetitions. These parameters were chosen based on a previous trial and error method to reduce spectrum noise. A 50x objective was used to tightly focus the laser on the sample surface. All spectra are baseline corrected and normalized to laser power. The supporting information section (Appendix $-C$ ) describes the procedure that was used to determine the analytical enhancement factor $(\mathrm{EF})$ value of the nickel nanonetwork.

\subsection{Statistics:}

All experiments were carried out thrice, and the data points were averaged, unless otherwise mentioned. The error bars represent standard deviation. 


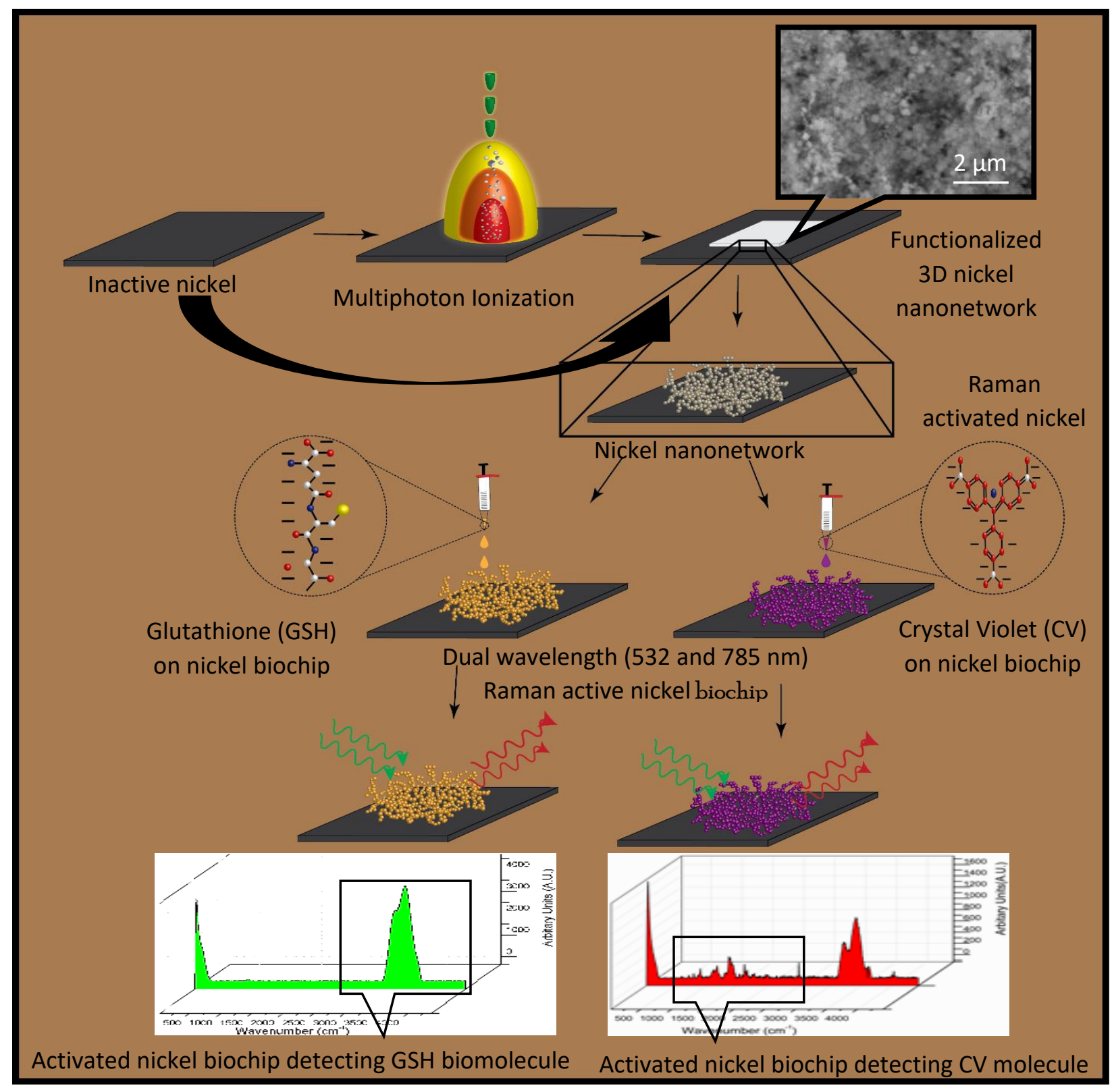

Figure 4-1: Functionalized 3D hybrid nickel nanonetwork fabrication on nickel biochip with dual wavelength Raman activation for GSH and CV detection

\subsection{RESULTS AND DISCUSSION:}

\subsubsection{Synthesizing Interdenominational Self-assembled 3D Hybrid Nickel Nanonetwork:}

The formation of self-assembled 3D nanostructures synthesized using ionic condensation has been previously investigated ${ }^{155}$. When a laser beam shines on a solid target, the material absorbs the photon energy. The absorption depth for metals is usually several tens of nanometers, which is a very small 
volume. Femtosecond lasers with extremely short pulse durations exhibit peak power as high as gigawatts. The small volume of material under irradiation absorbs the energy in a very short time, and thus undergoes a swift transformation from an initial cold solidified state to a high energy plasma state, where time-dependent ionization and material recombination occurs ${ }^{61}$. At the interface of the plasma and ambient atmosphere, more complicated chemical reactions occur between the ionized species and surrounding atmosphere molecules. This involves the formation of oxides and compounds having specific material properties (morphology, size, and oxide phase). Here, it is expected that the synthesized species, in the form of ions, atoms, or clusters, would strongly react with oxygen molecules in air to form $\mathrm{Ni} / \mathrm{NiO}$ droplets, which are quenched rapidly. Because of the high kinetic energy of the expanding plasma, larger cooler droplets will be ejected out of the plasma, forming a self-assembled 3D hybrid nickel nanonetwork. By varying the laser parameters, the temperature of the plasma can be varied, which in turn alters the size, morphology, and composition of the formed 3D nanonetwork. Therefore, the properties of the 3D nanonetwork can be tuned to a certain extent.

\subsubsection{Structure, morphology, and physical property analysis of the synthesized nanonetwork:}

The controllability of the synthesized 3D self-assembled hybrid nickel nanonetwork on the nickel surface is demonstrated in the inset of Figure 4-2. Two types of particle shapes were observed, spherical particles and cubic particles, as shown in the inset of Figure 4-2. Nanonetworks generated by an ultra-short pulsed laser (UPL) are known to vary in size depending on the experimental conditions ${ }^{156}$. In addition, it was observed here that cuboidal particles were smaller in size, while most of the larger particles exhibited a spherical shape. The average particle sizes consistently decreased with increasing dwell time (number of incident laser pulses).

AFM contrast studies were carried out to analyze the height and phase composition of the synthesized 3D hybrid nickel nanonetwork. In comparison to the SEM images shown in the inset of Figure 4-2, the AFM images remain relatively uniform regardless of the 3D nanonetwork. It is worth noting that the sharp- 
edged nickel nanonetwork imaged in the insert of Figure 4-2 appears to be smooth in the AFM scan. Although, the AFM scan revealed areas of varying density of nanofibrous structures, the scan did not reveal the surface morphology of the 3D nickel nanonetwork.

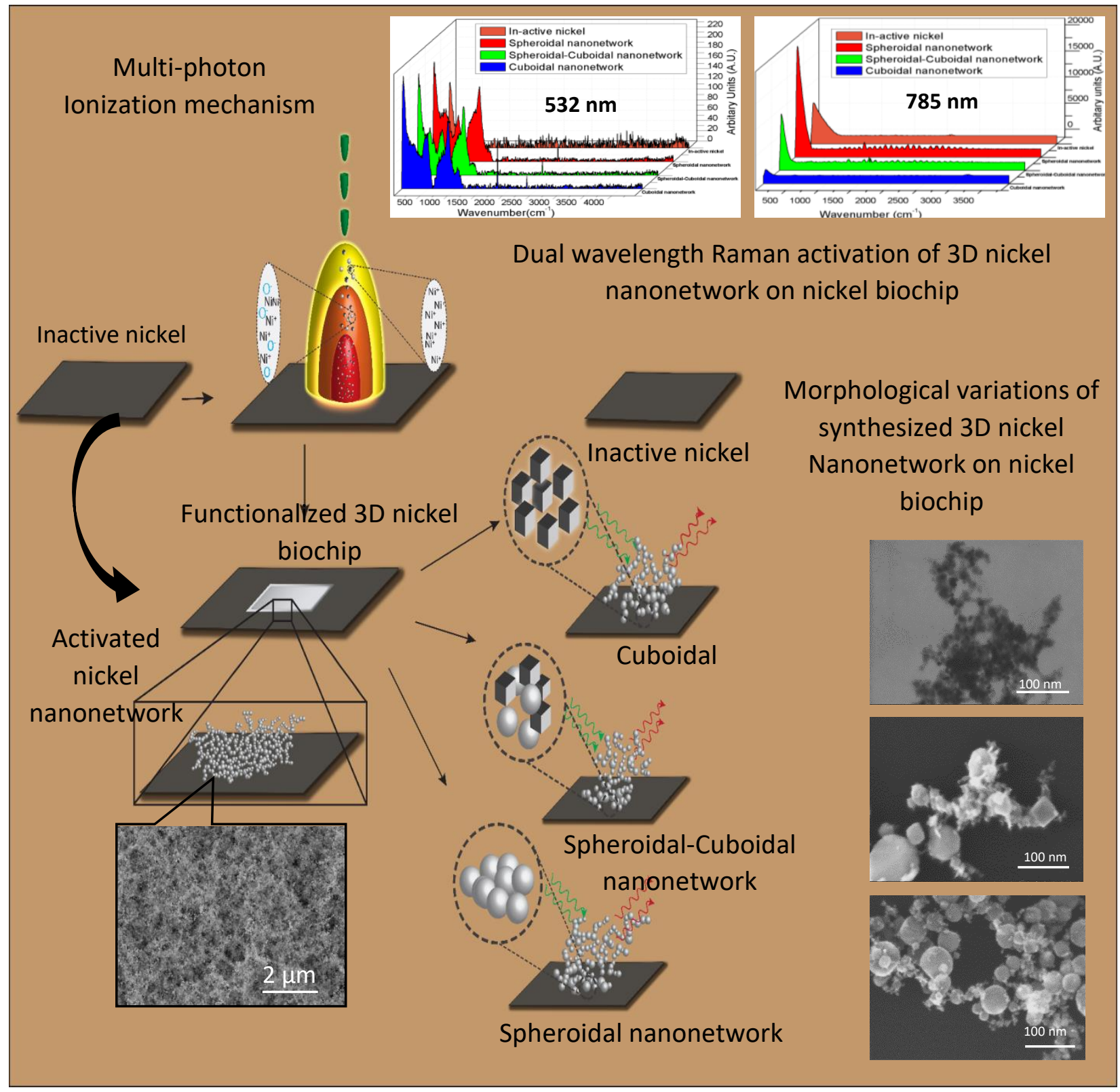

Figure 4-2: Synthesis of 3D nickel nanonetwork on nickel substrate using multi-photon ionization mechanism and subsequent Raman self-activation. The multiphoton ionization created cuboidal, spheroidal-cuboidal and spheroidal morphology nanonetwork 
Thus, it is increasingly difficult to image the 3D flexible nanonetwork by using AFM and absorption/excitation of SERS. This uncertainty gets compounded with complex geometries observed in cuboidal and spheroidal-cuboidal nanonetwork SEM images, as shown by the cubic-shaped nanoparticles in the inset of Figure 4-2. Constructive interference between the incident and reflected light can enhance the electro-magnetic effect with no apparent change in the plasmon resonance ${ }^{157}$. The plasmon width and red shifting are sensitive to the size and morphology of the suspended nanonetwork ${ }^{158}$. Therefore, the flexibility of the 3D nanonetwork with the suspended nanoparticles is affected by the network depth and porosity. The AFM height scans in the inset of Appendix Figure C-S1(A) shows that the cuboidal nanonetwork is denser than the spheroidal nanonetwork. The topographic image revealed that the interacting forces between the AFM tip and nanonetwork are not magnetic and involve the combined effect of electrostatic and Van der Waals interactions. The XRD spectrum from the inset of Figure C-S1(C) shows a significant line broadening, which is a characteristic phenomenon of nickel/nickel oxide nanoparticles. The spectrum clearly shows that the nanonetwork is of a crystalline nature. The crystallite size was estimated from the XRD pattern using by Scherrer's equation, where the shape factor $K=0.9, X-$ ray wavelength of Copper $(\mathrm{Cu}) \mathrm{K} \alpha=1.54 \AA$, Bragg diffraction angle is $\theta$, and $\beta$ is the FWHM of the respective diffraction peak. The average crystallite size of the synthesized self-assembled 3D hybrid nickel nanonetwork was found to be approximately $50 \mathrm{~nm}$, which is much larger than that observed from the TEM images in the inset of Figure 4-2.

The energy dispersive X-ray (EDX) analysis using FESEM on individual nanoparticles detected only elemental nickel and oxygen exclusive of substrate signals. The ratio of $\mathrm{Ni}$ and $\mathrm{O}$ is almost 1:1, supporting the formation of NiO. Further analysis did show that the composition remained consistent irrespective of particle size and shape. The line EDX images in the scanning-transmission (STEM) mode for the selected nanonetwork are showcased in the inset of Figure 4-3C. The XRD diffraction patterns of Ni and NiO appear to be in simple cubic and rhombohedral structures, with elemental Ni present in a significant amount. The 
reflection peaks for $\mathrm{Ni}$ is indexed entirely to face-centered-cubic (fcc) structure \{space group: Fm3m (225)\} of Ni nanocrystals having unit cell dimension: $a=3.5234 \AA$. Three peaks at $2^{\theta}=44.5,51.9$, and 76.5 correspond to the diffraction from the $\{111,200,220\}$ facets, respectively, and the peaks at $2^{\theta}=37.28$, $43.28,62.88,75.28$ and 79.48 correspond to the diffraction from the $\{111,200,220,311,222\}$ facets, respectively.

\subsubsection{Self-assembled nanonetwork material properties and SERS Enhancement:}

As shown in the inset of Figure 4-3, the Raman spectrum of crystalline Ni-NiO shows several bands in the probed region above $400 \mathrm{~cm}^{-1}$. There are four peaks, $65 \mathrm{~cm}^{-1}, 1333 \mathrm{~cm}^{-1}, 1775 \mathrm{~cm}^{-1}$ and $2300 \mathrm{~cm}^{-1}$. The peaks around $65 \mathrm{~cm}^{-1}, 1333 \mathrm{~cm}^{-1}$, and $2300 \mathrm{~cm}^{-1}$ can be assigned to the 1st magnon (1MO), 2nd (2MO), and 4th (4MO) mode vibrations based on the literature ${ }^{98,108,159}$. The 3rd-magnon mode identified at 1776 $\mathrm{cm}^{-1}$ in the inset of Figure 4-3A is consistently observed for a cuboidal nanonetwork and has never been reported in the existing literature.

Similarly, the peak found at $490 \mathrm{~cm}^{-1}$ is assigned to the one-phonon longitudinal optical (LO) and transverse optical (TO) mode of $\mathrm{NiO}$, based on previous research by Ghandhi et al. ${ }^{159}$, and the peak at 870 $\mathrm{cm}^{-1}$ is assigned to the two-phonon (2LO) mode based on the literature ${ }^{108}$. The first order Raman scattering peak at $490 \mathrm{~cm}^{-1}$ is slightly diminished and shifted when compared with the second order peak found at $870 \mathrm{~cm}^{-1}$, implying a high nickel to nickel oxide vacancy concentration. 

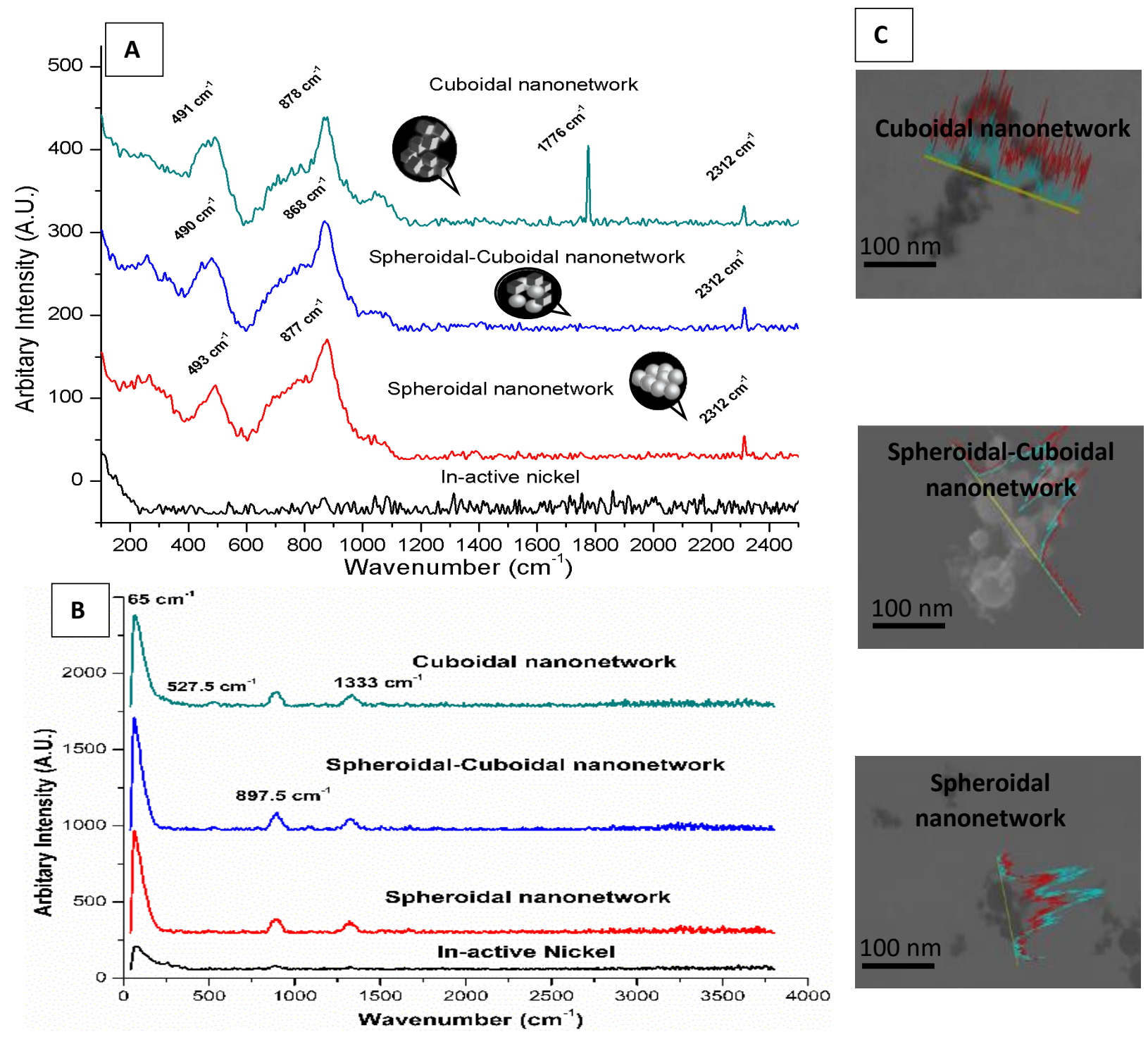

Figure 4-3: SERS activated nickel nanonetwork at cuboidal, spheroidal-cuboidal and spheroidal nanonetwork morphology along with inactive nickel substrate at A) $532 \mathrm{~nm}$ Raman laser excitation B) $785 \mathrm{~nm}$ Raman laser excitation and C) compositional analysis of corresponding nickel nanonetworks

\subsubsection{Raman scattering effect of CV on Nickel nanonetwork:}

Crystal violet is a common cationic dye with a symmetric molecular structure. The native bulk nickel substrate has no response to the presence of the CV dye. However, the dye-coated nickel nanonetwork evidently showcased the characteristic peaks for the associated dye at concentrations ranging from $10^{-3}$ $\mathrm{M}$ to $10^{-12} \mathrm{M}$. The SERS response to $\mathrm{CV}$ dye, enhanced by the nickel nanonetwork at the 532-nm wavelength of the Raman laser, is shown in the inset of Figure 4-4. The spectrum obtained have relative 
shifts in the peak positions and intensity owing to strong surface reactions induced between the interacting CV dye molecule and nanoparticles present within the nanonetwork. This in turn translates to the selective enhancement of dye peaks, which are not observed with other nanomaterials. The Raman spectra of CV detected through the nanonetwork are shown in the inset of Figure C-S2. In general, the vibrations of the central carbon $\left(\mathrm{C}+\right.$ ) phenyl bonds are assigned from $100-400 \mathrm{~cm}^{-1}$ and phenyl vibrations are above $400 \mathrm{~cm}^{-1}$. Yamada et al. used single crystalline metal oxide (e.g., $\mathrm{NiO}$ and $\mathrm{TiO}_{2}$ ) substrates and observed enhanced Raman response of pyridine. The observed results indicate the dependency of the characteristic Raman intensity peaks of pyridine on metal oxides employed for SERS enhancement. The Raman spectrum of a given sample varies depending on the type of metal oxide adopted ${ }^{160}$. The efficiency of the charge transfer process completely depends on the vibronic coupling of the conduction and valence bands induced by $\mathrm{NiO}$ nanoparticle chains. When the dimensions of the synthesized nickel nanonetwork become comparable to the size of the Bhor radius, quantization occurs. This in turn forces a weak Raman response at energy transition levels in conduction, valance bands, and vibronic coupling.

Both millimolar and micromolar concentrations of CV dye induced Raman response until $1700 \mathrm{~cm}^{-1}$. A sudden shift is observed in the Raman spectra collected at nano and picomolar concentrations with active peaks beyond $3000 \mathrm{~cm}^{-1}$. The peak observed at both $900 \mathrm{~cm}^{-1}$ and $3200 \mathrm{~cm}^{-1}$ show aromatic (C-H) group in the out-of-plane bend and stretch mode type molecular vibrations. This strong Raman response in both modes have never been reported. Lombardi and Birke asserted that an enhancement factor above $10^{4}$ cannot solely result from charge transfer. The enhancement factor achieved here is in the order of $10^{9}$. The strong enhancement observed here could be a combined effect of the charge transfer and "hot spot" 142 .

\subsubsection{Realizing biomolecule detection on nickel biochip:}

GSH is an abundant non-protein-based thiol source with an intracellular concentration of $\sim 10 \mathrm{mM}$ present in most mammalian cells ${ }^{161}$. Glutathione levels present in cells can be related to specific diseases, such as 
cancer, Alzheimer, and Parkinson's, as well as diseases caused by aging ${ }^{162,163}$. A variety of screening methods have now been developed to detect GSH levels, for instance, electrochemical analysis, fluorescence spectrometry ${ }^{164-166}$, colloidal-particle-based colorimetric assays ${ }^{147}$, enzyme linked immunosorbent assay (ELISA), and UV/vis spectrometry. These methods however yielded limited sensitivity with only $\mu \mathrm{M}$ and $\mathrm{nM}$ limit of detection (LOD) performed on substrates with the constraint of conducting an external analysis and quantification ${ }^{167-171}$. On a substrate, GSH has a natural tendency to promote the aggregation of noble metal nanoparticles, which usually result in a stronger electromagnetic effect. Therefore, SERS emerged recently as a new method of GSH detection on substrates. Metal colloids such as gold and silver are generally considered to have better SERS enhancement with high reproducibility and manipulability. However, owing to colloidal aggregation induced by the analytes, spectra were highly unstable and reproducibility was poor ${ }^{172-174}$. 

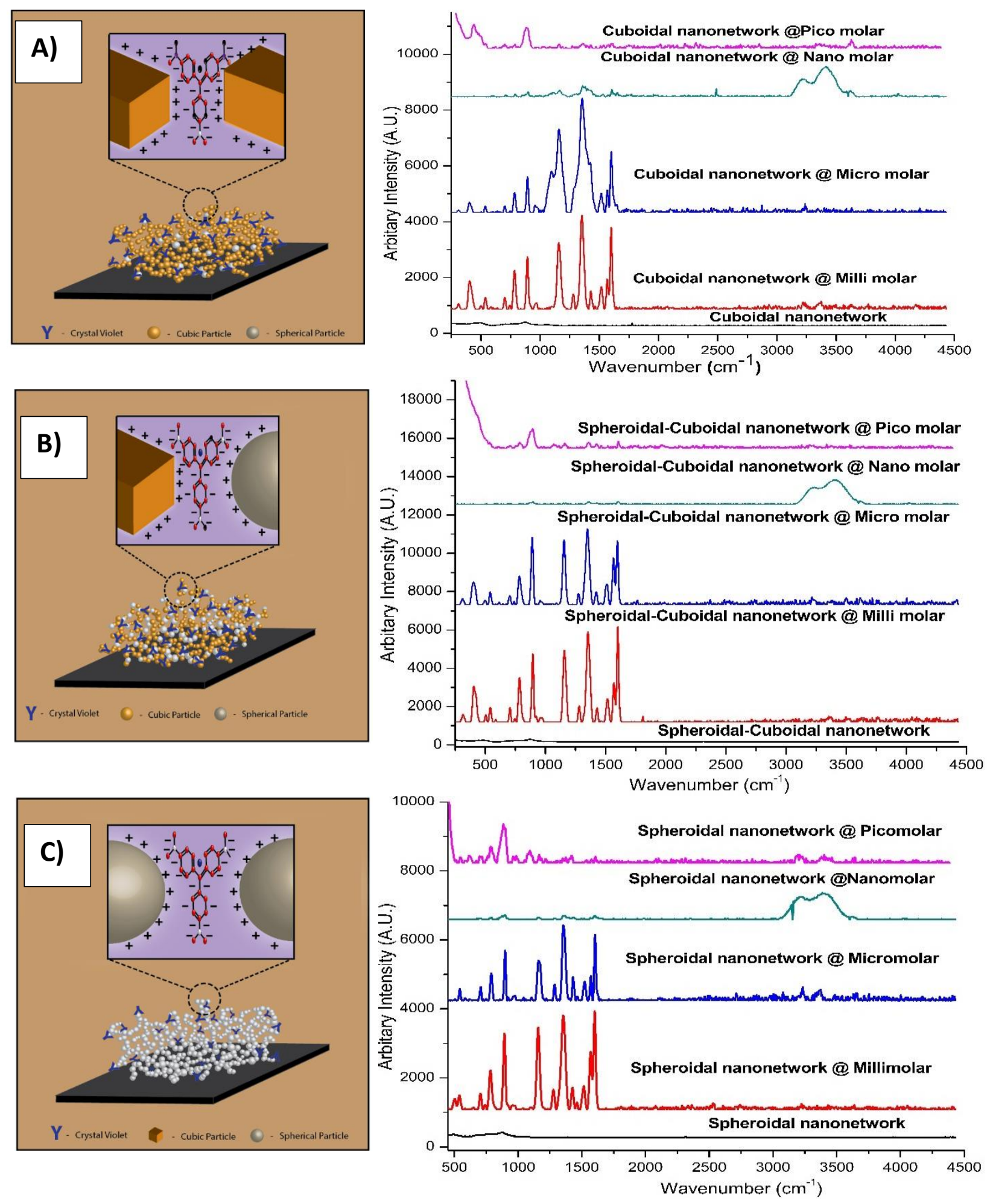

Figure 4-4: Functionalized Raman activation of synthesized nanonetwork with crystal violet from millimolar to picomolar concentration for $532 \mathrm{~nm}$ Raman laser irradiation at A) Cuboidal nanonetwork B) Spheroidal-Cuboidal nanonetwork C) Spheroidal nanonetwork 
The capability of the self-assembled 3D hybrid nickel nanonetwork for GSH biomolecule detection was tested. Figures 4-5 and Appendix Figure C-S3 highlight the concentration profile of GSH where the response of GSH increases as the concentration decreases from the millimolar to picomolar range. The strongest spectra intensity was observed at picomolar concentrations for 532 and $785 \mathrm{~nm}$ Raman laser excitations. The detection limit of GSH biomolecule was at least $1 \mathrm{pM}$. A few of the most prominent bands for GSH biomolecule were observed between 890 and $3300 \mathrm{~cm}^{-1}$ for the $532 \mathrm{~nm}$ Raman laser wavelength in the inset of Figure C-S3. In general, it is expected that most of the collected Raman spectra and SERS of polypeptides can be assigned based on their corresponding spectral amino acids ${ }^{175}$.

Thus, those bands for GSH biomolecule may be associated with vibration modes of the sulfur atom present in the amino acids cysteine (Cys). The intensity of these Raman bands at different concentrations of GSH and the large enhancement for $\mathrm{C}-\mathrm{S}$ stretching modes indicate that the sulfur atom present in GSH interacts strongly with the nickel nanonetworks. The strong spectral peaks of GSH appear around 3400 $\mathrm{cm}^{-1}$ for the $532 \mathrm{~nm}$ Raman laser excitation, representing $\mathrm{O}-\mathrm{H}$ vibrations of carboxylic acids. The obtained signal-to-noise $(S N R)$ ratio is 8.75 , which is lower than that $(S N R=3)$ reported by Larsson et al. and Deregowska et al., who used a colloidal gold substrate as a primary source to enhance Raman peaks ${ }^{168,176}$. The Raman laser irradiation at $785 \mathrm{~nm}$ significantly enhanced the carboxyl group vibrations at 910 and $1320 \mathrm{~cm}^{-1}$. The carboxylate groups can be assigned to the $\mathrm{C}^{-\mathrm{COO}^{-}}$stretching vibrations and symmetry, respectively. Meanwhile, the bands originating from the $\mathrm{N}$-terminal group are only present at picomolar concentrations for the $532 \mathrm{~nm}$ Raman laser irradiation in the spheroidal-cuboidal nanonetwork. This weak band at $1055 \mathrm{~cm}^{-1}$ is first assigned to the amino group, suggesting that GSH interacts with the nickel nanonetwork and not only through the carboxylate group, as suggested earlier by Meng Lv et al. ${ }^{177}$, but other functional groups may also interact with $\mathrm{Ni} / \mathrm{NiO}$. The band appearing at $1633 \mathrm{~cm}^{-1}$ can be assigned to the first amide vibration. Their relative Raman intensity peaks are consistently weakly observed in all concentrations of GSH from the inset of Figure S3 and in Table B S2. 

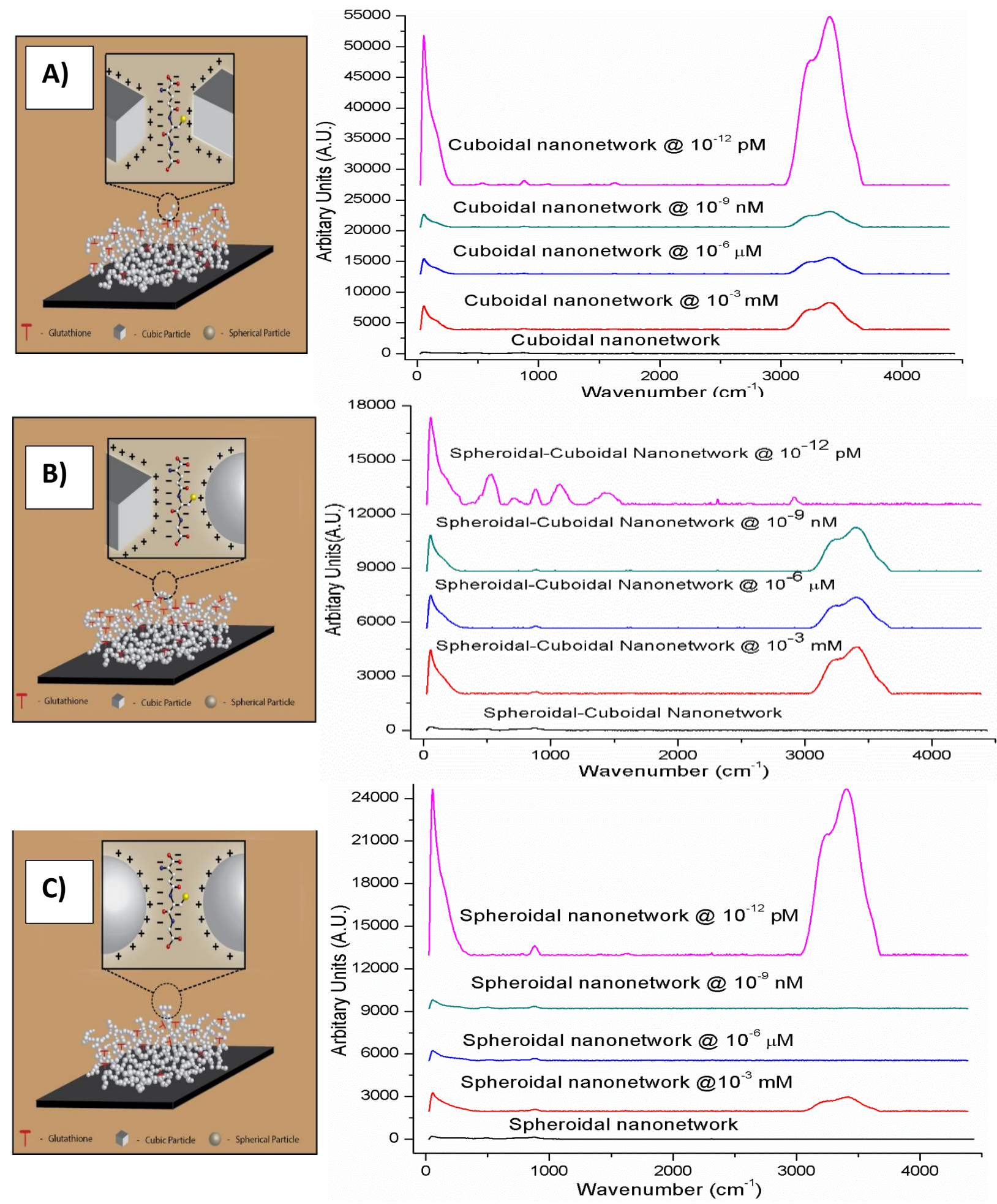

Figure 4-5: Functionalized Raman activation of synthesized nanonetwork with glutathione (GSH) biomolecule from millimolar to picomolar concentration for $532 \mathrm{~nm}$ Raman laser irradiation at A) Cuboidal nanonetwork B) Spheroidal-Cuboidal nanonetwork C) Spheroidal nanonetwork at 532 nm Raman laser irradiation 
The sharp corners and edges of the cuboidal nanoparticles in the 3D network provided many sites that readily trap the analyte molecules. These aggregated molecules form "hot spots," and this subsequently translates to large SERS enhancement factor per surface molecule. It is well-established that these "hot spots" contribute to SERS, and the enhancement due to the self-assembled nanostructures is about five orders of magnitude higher than that induced by isolated nanoparticles ${ }^{178,179}$. Although self-assembled nanostructures have been widely studied, the fabrication of controllable and reproducible "hot spots" remains a major challenge. Recently, He et al. demonstrated high SERS enhancement by fabricating arranged silver nanoparticles and gold nanorods embedded in polyvinyl alcohol (PVA) nanofibers through electrospinning ${ }^{180}$.

The SERS spectrum at picomolar concentrations of GSH is strong and presented a variety of peaks up to $4000 \mathrm{~cm}^{-1}$. In contrast, other methods normally generated only few weak Raman excitation peaks if active at picomolar concentrations. The high sensitivity of the 3D hybrid nickel nanonetwork can be attributed to the multiple E-field enhancements from nanoparticle crevice gaps, and to the mutual charge transfer between the dye molecule and the nanoparticle. For example, single crystalline Ag nanocubes generated using the polyol method yielded a $10^{5}$ fold enhancement of Rhodamine $6 \mathrm{G}$ analyte owing to a large number of generated Ag nanocubes acting as hotspot ${ }^{179}$. When compared to the traditional approach of using noble metal with gap plasmonics for SERS enhancement, the hybrid nickel nanonetwork provided multiple "hot spots", leading to increased peaks of the spectrum.

\subsubsection{Establishing nano-biosensor sensitivity and limit of detection:}

Most established approaches (physical and chemical) employ prolonged incubation of target molecules and heating of the synthesized SERS platform to enhance the sensitivity and selectivity ${ }^{181,182}$. This entire process is eliminated with the 3D hybrid nickel nanonetwork, thereby providing instantaneous detection. The 3D nickel nanonetwork was used to test glutathione concentration varying from $1 \mathrm{mM}$ to $1 \mathrm{pM}$. The 
characteristic intensity peak of GSH is observed at $3300 \mathrm{~cm}^{-1}$, and at $1600 \mathrm{~cm}^{-1}$ for CV for the $532 \mathrm{~nm}$ Raman laser excitation, as shown in the inset of Figure 4-6 and in Table B S2. The cuboidal nanonetwork demonstrated the strongest enhancement at all GSH biomolecule concentrations from $1 \mathrm{mM}$ to $1 \mathrm{pM}$. The spheroidal nanonetwork presented the least enhancement at higher ( $1 \mathrm{mM})$ dye concentrations but did not show any enhancement at both $\mu \mathrm{M}$ and $\mathrm{nM}$ GSH concentration suggesting that the enhancement observed at $\mathrm{mM}$ is due to analyte saturation only. However, a moderate enhancement was noticed at 1 pM GSH concentration indicating that the enhancement is due to charge transfer mechanism.

However, the spheroidal-cuboidal hybrid nanonetwork, which showed moderate enhancement at higher (1 mM) GSH biomolecule concentrations, presented the least enhancement at $1 \mathrm{pM}$, compared with the cuboidal and spheroidal nanonetwork. This reduced enhancement at high (1 mM) concentrations is influenced by a variety of factors, such as the nanomorphology and material oxide phase composition of the nickel nanonetworks. Jiwon Lee et al. reported $10 \mathrm{pM}$ sensitive GSH biomolecule detection by varying the noble metal composition present in the nanostructure, thus implying the significance of material 


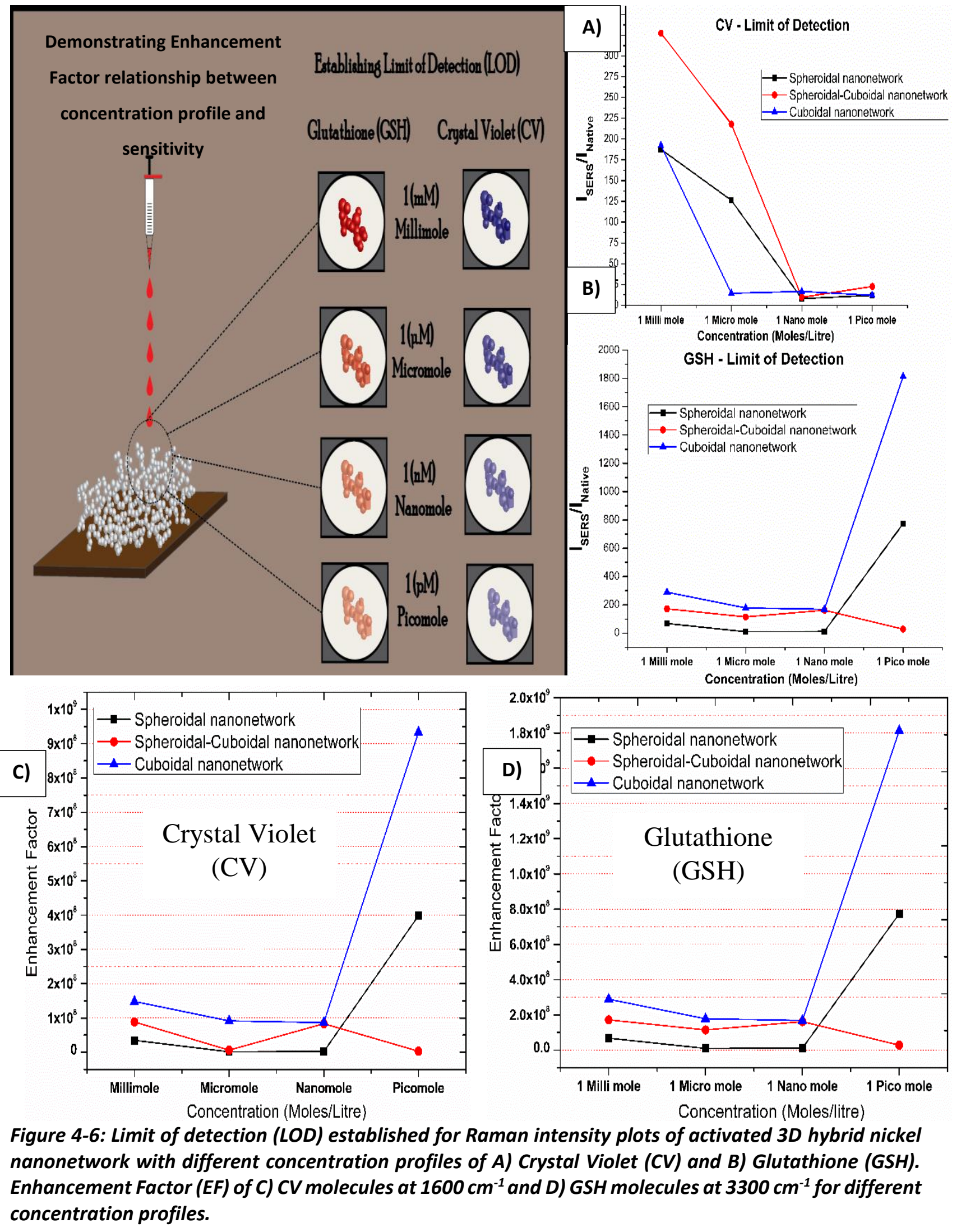




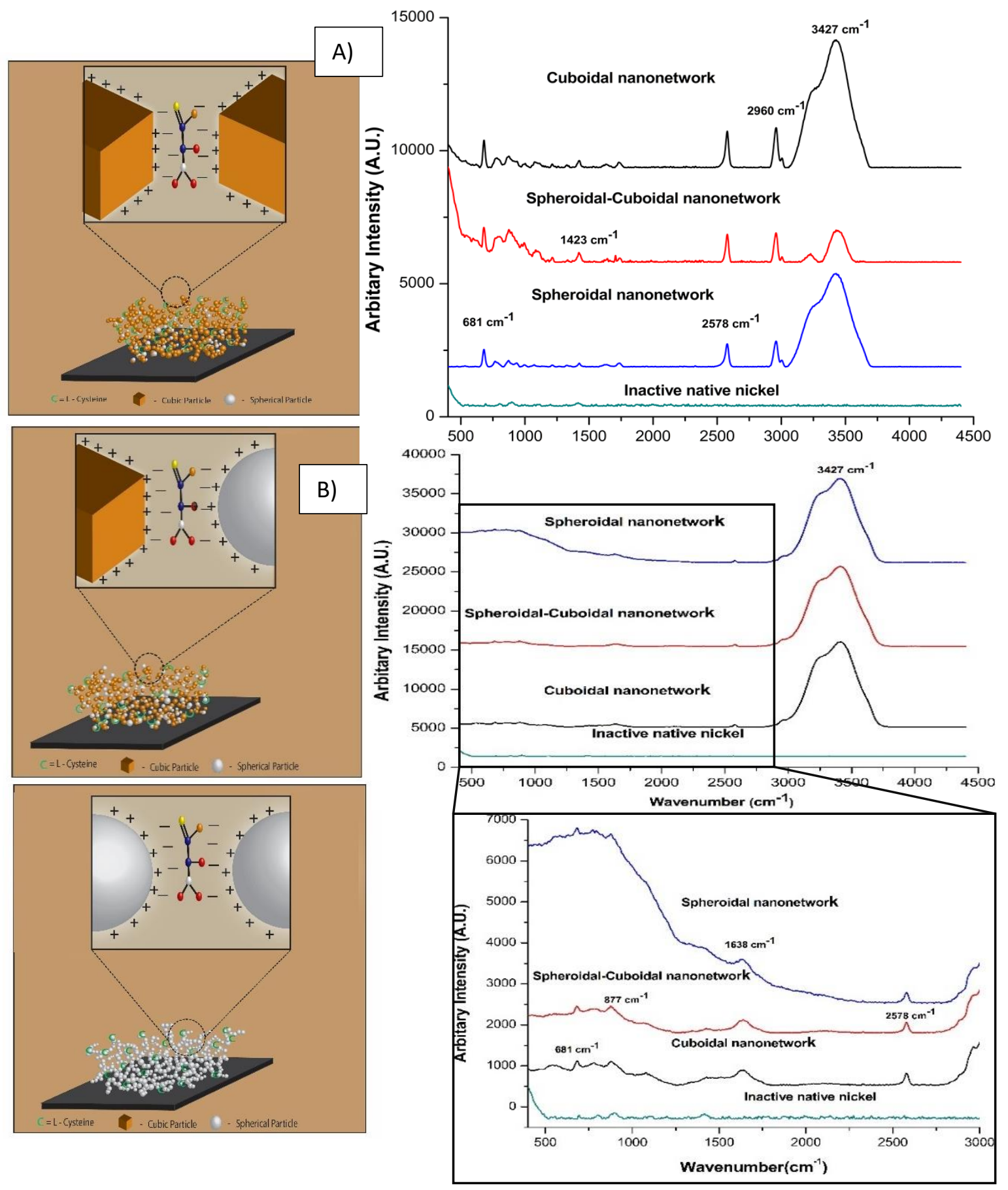

Figure 4-7: Functionalized Raman activation of synthesized nanonetwork with L-Cysteine (L-Cys) biomolecule for $532 \mathrm{~nm}$ Raman laser irradiation for Cuboidal nanonetwork, Spheroidal-Cuboidal nanonetwork and Spheroidal nanonetwork A) $1 \mathrm{mM}$ and B) $1 \mu \mathrm{M}$ concentration at $532 \mathrm{~nm}$ Raman laser irradiation.

composition in biomolecule sensing and enhancement ${ }^{183}$. Similarly, Guan et al. reported only a micromolar sensitive GSH biosensor using cuboidal gold nanoparticles ${ }^{184}$. 
Nevertheless, the nickel nanonetworks synthesized here could sense GSH biomolecules up to concentrations of $1 \mathrm{pM}$ with an enhancement factor on the order of $10^{9}$. To the best of our knowledge, this is the highest enhancement factor reported thus far with Raman spectra detection for GSH at concentration as low as $1 \mathrm{pM}$ using non-noble-metal nanomaterials.

\subsubsection{Accomplishing L-Cysteine and L-Methionine detection on nickel nano chips:}

In order to show versatility of the nickel nanobiosensor synthesized, aqueous solutions of L-Cysteine (LCys) and L-Methionine (L-Met) biomolecules were tested individually on nickel nanobiosensor synthesized at $532 \mathrm{~nm}$ Raman laser excitations. Cysteine is responsible for stabilization of secondary protein structures and functions as a powerful antioxidant protecting the body against radiation, while Methionine aids in initiating the process of translation as messenger RNA ${ }^{185}$. The inactive native nickel substrate did not induce any Raman activity. However, the synthesized nickel nanonetwork induced strong SERS spectra response on each amino acid/biomolecules observed at both 1 millimolar and 1 micromolar concentrations which are presented in the inset of Figure 4-(7 and 8). The collected spectra for L-Cys and L-Met are unique with enhancement/activation of bands exclusive to the biomolecule tested.

Most prominent bands for L-Cys biomolecule was observed at 681, 877, 1423, 1638, 2578, 2960 and 3427 $\mathrm{cm}^{-1}$ for $532 \mathrm{~nm}$ Raman laser wavelength at both $1 \mathrm{mM}$ and $1 \mu \mathrm{M}$ concentration highlighted in the inset Figure 4-7. The series of bands observed at $681 \mathrm{~cm}^{-1}, 877 \mathrm{~cm}^{-1}$ and $1423 \mathrm{~cm}^{-1}$ are assigned to C-S, C-C stretching vibration and $\mathrm{CH}_{2}$ bending vibration pertaining to L-Cys based on the work by Podstawka et $\mathrm{al}^{185}$. In addition, a series of sharp and strong bands were also observed at 1638,2578 and $2960 \mathrm{~cm}^{-1}$. The band at $1638 \mathrm{~cm}^{-1}$ can be assigned to carbonyl $\mathrm{C}=\mathrm{O}$ asymmetric stretching vibration, while $\mathrm{S}-\mathrm{H}$ stretching vibration can be assigned at $2578 \mathrm{~cm}^{-1}$ and lastly $\mathrm{C}-\mathrm{H}$ stretching vibration assigned at $2960 \mathrm{~cm}^{-1}$ as earlier reported by Pawlukojć et al ${ }^{186}$. The nanonetwork distinctively induced sharp S-H stretching at $2578 \mathrm{~cm}^{-1}$ within $6 \mathrm{~cm}^{-1}$ signifying the complete absence of hydrogen bonding as reported by Edsall et al ${ }^{187}$. 
Additionally, broad band was observed at $3427 \mathrm{~cm}^{-1}$ which can be assigned to $\mathrm{NH}_{3}$ asymmetric stretching vibration of L-Cys.

Likewise, SERS spectra of L-Met activated on nickel nanonetworks presented discernable peaks at 641, $704,880,1426$ and $2929 \mathrm{~cm}^{-1}$ is presented in the inset Figure 8. Based on the work by Podstawka et al ${ }^{185}$., the band at 641 and $704 \mathrm{~cm}^{-1}$ are assigned to both carboxyl COO- and C-S symmetric stretching vibrations. The bands at 880,1426 and $2929 \mathrm{~cm}^{-1}$ is assigned to $\mathrm{CH}_{2}$ rocking, $\mathrm{CH}_{2}$ bending and $\mathrm{CH}_{2}$ asymmetric stretching vibrations respectively from Zhu et al and Pandiarajan et al ${ }^{188,189}$. The strong peak at $2929 \mathrm{~cm}^{-1}$ assigned as $\mathrm{CH}_{2}$ asymmetric stretching vibrations had difference in peak height exhibited with L-Met for millimolar and micromolar concentrations. Also, the broad bands at 3233 and $3421 \mathrm{~cm}^{-1}$ can be assigned to stretching modes of $\mathrm{N}-\mathrm{H}$ and $\mathrm{NH}_{3}$ vibrations of $\mathrm{L}-\mathrm{Met}^{190}$.
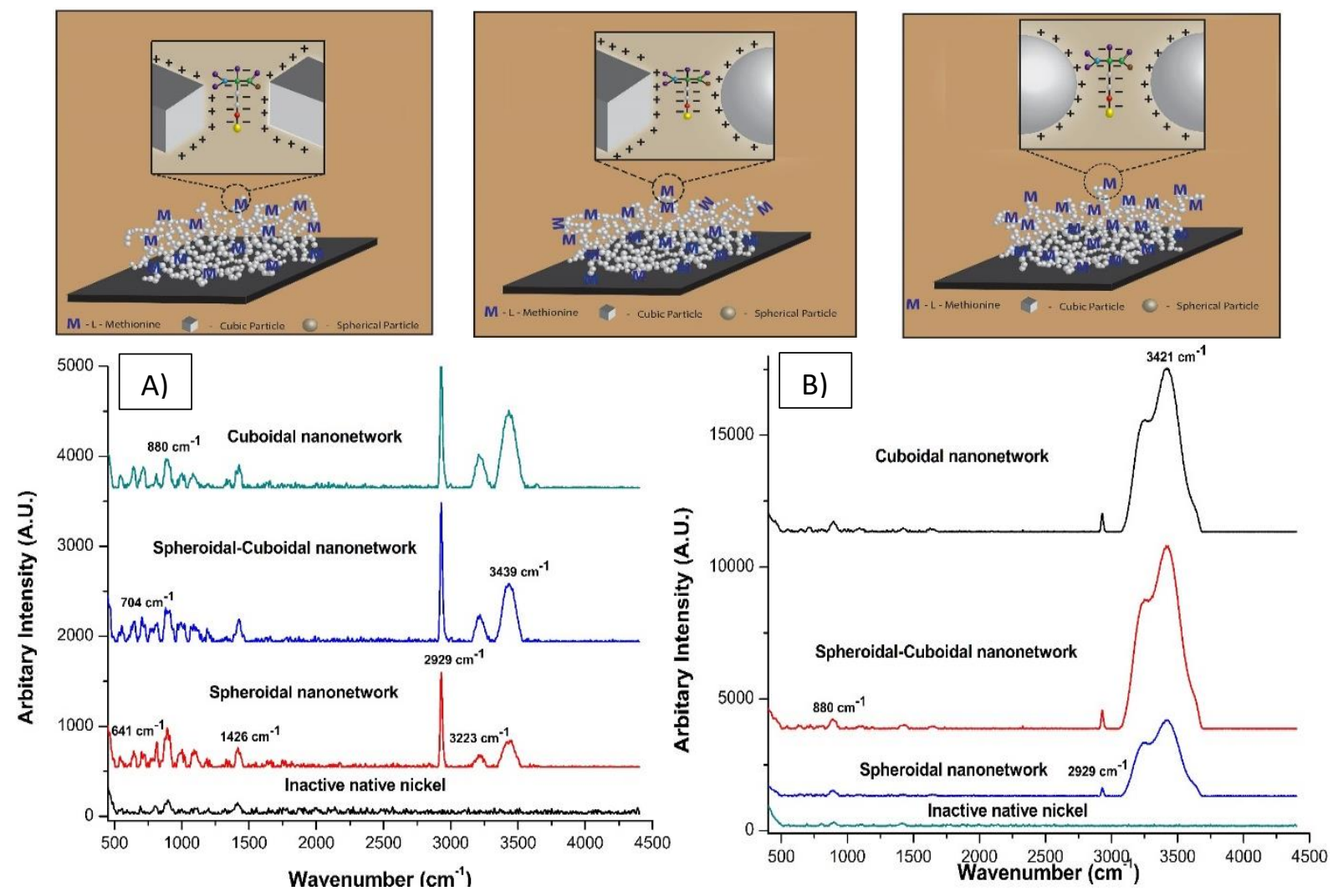

Figure 4-8: Functionalized Raman activation of synthesized nanonetwork with L-Methionine (L-Met) biomolecule for $532 \mathrm{~nm}$ Raman laser irradiation for Cuboidal nanonetwork, Spheroidal-Cuboidal nanonetwork and Spheroidal nanonetwork A) $1 \mathrm{mM}$ and B) $1 \mu \mathrm{M}$ concentration at $532 \mathrm{~nm}$ Raman laser irradiation. 


\subsubsection{Accomplishing GSH sensing in complex biological environment detection on nickel nano}

chips:

Cell culture medium contain all the elements that cells need for growth, sustained maintenance in laboratory environment mimicking real body conditions. The aim of this study is to find the selectivity of synthesized nickel nanonetwork in sensing GSH in complex biological environment.
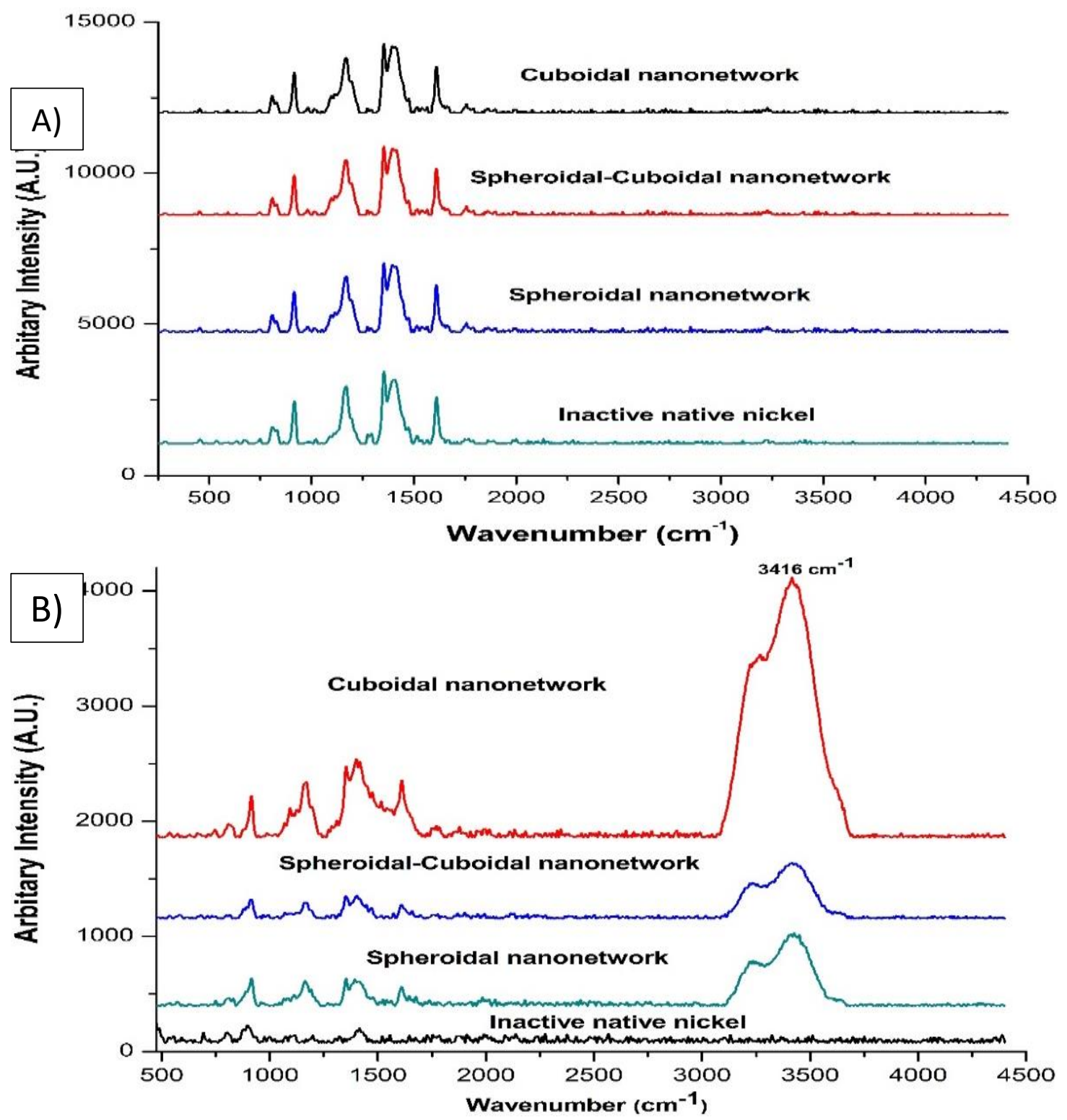

Figure 4-9: Functionalized Raman activation of synthesized nanonetwork Cuboidal nanonetwork, Spheroidal-Cuboidal nanonetwork and Spheroidal nanonetwork at A) complex biological environment (Cell culture medium) and B) $1 \mu \mathrm{m}$ concentration of GSH added to complex biological environment (Cell culture medium) at $532 \mathrm{~nm}$ Raman laser irradiation 
The SERS response to complex biological environment on nickel nanonetwork and inactive nickel substrate is presented in the inset of Figure 4-9A. Both the nanonetwork and inactive nickel substrate produced equal Raman response to the complex biological environment. Nevertheless, when $1 \mu \mathrm{M}$ concentration of GSH biomolecule when added to this complex biological environment, it induced an idiosyncratic Raman response to the already synthesized nickel nanonetwork. A strong spectral peak were noticed at $3416 \mathrm{~cm}^{-1}$ for all nickel nanonetworks at $532 \mathrm{~nm}$ Raman laser excitation along with other peaks contributed by the complex biological environment also. This peak represents $\mathrm{O}-\mathrm{H}$ vibrations of carboxylic acids present in GSH diluted in a complex biological environment. Thus, this study showcases the selectivity of synthesized nickel nanonetworks while sensing GSH in complex biological environment.

\subsection{CONCLUSIONS:}

In summary, we have introduced a tunable "non-noble metal" based SERS active nano-biosensor chip for biomolecule detection using self-assembled 3D hybrid nickel nanonetworks. The nickel biochip synthesized with tunable physiochemical characteristics was functionalized by multiphoton ionization nanomaterials synthesis. With this nanobiosensor, Raman spectra were obtained for detection of CV dye molecules and GSH molecules at two Raman excitation wavelengths, namely $532 \mathrm{~nm}$ and $785 \mathrm{~nm}$. The results showed a limit of detection of one picomolar (1 pM) for both GSH and CV with an enhancement factor of $1.8 \times 10^{9}$ and $9.3 \times 10^{8}$. The versatility of this nanobiosensor was examined with biomolecules such as L-Cys and L-Met at $1 \mathrm{mM}$ and $1 \mu \mathrm{M}$ concentrations. In addition, the selectivity of the biosensor is now reported using $1 \mu \mathrm{M}$ concentration of GSH in a cell culture medium which mimics complex biological environment. This is the first time a "non-noble metal" based ultra-sensitive nano-biosensor platform was demonstrated. With high sensitivity and strong signal output at $532 \mathrm{~nm}$ and $785 \mathrm{~nm}$ excitations, this approach is promising for both in-vitro and in-vivo sensing applications. 


\section{CHAPTER 5}

\section{ATOMIC ANTENNAS ON PORTABLE QUANTUM STRUCTURES - A NON -TERS APPROACH}

Tip-Enhanced Raman spectroscopy (TERS) is a powerful technique that integrates unique Raman molecular vibrational finger-print at sub-nanometer scale within a single molecule limit. Current work in TERS primarily focusses on plasmonic nanomaterials suffer from non-portability of TERS probes, molecule selectivity and biocompatibility when used in an in-vitro tumor environment. Therefore, this research work is open to both stand-alone biomolecule sensing and for in-vitro cancer diagnostics. Herein, we introduce two quantum probes with and without atomic scale antennas for reliable label-free cancer detection for single molecule with Raman enhancement factor that reach up to $10^{12}$ sensitivity. These quantum probes synthesized in this study when observed had an exponential increase in Raman enhancement at femtomolar concentration (10-

${ }^{15} \mathrm{M}$ ) with Raman active crystal violet and Rhodamine $6 \mathrm{G}$. The uptake of the portable quantum probes inside the cell is through passive endocytosis mechanism. The results derived for the invitro sensitivity also demonstrate the acute and precise sensitivity of the atomic scale antennas present within the quantum probes so as to detect and differentiate cancer from those of fibroblast cells interacting in a cellular environment. This unlocks the potential of quantum probes with atomic antennas to mimic TERS by addressing the lack of label-free and portability of these probes for application at the desired site of interest. To the best of our knowledge, this is the first report of such a phenomenon with nm spatial resolution. We are confident that our results will open up new perspectives in the field of optical cancer diagnostics with nanometer resolution. 


\subsection{INTRODUCTION:}

It is pertinent to understand the nature of molecule-surface interactions at Angstorm/atomic scale to the development of self-assembled structures for nanoscale electronics, molecular biosensors and other photonic devices 191,192 . Chemical or molecule identification and sensing by optical means for biological applications to Single Molecule (SM) sensitivity is a challenging task. For instance, super-resolution fluorescence microscopy can be used to image biological samples below diffraction limit, but the use of targeted fluorescence labels and tags prevents its use for real-time biosensing application in their native state ${ }^{193}$. To date, Tip Enhanced Raman Scattering (TERS) is one such optical sensing technique that can work below the diffraction limit and has also the potential to overcome the aforesaid limitation only to be successfully used for all label-free biological sensing applications ${ }^{194}$.

The Raman enhancement observed is primarily through an electromagnetic enhancement when a sharp metal tip-apex of the probe that comes in close contact with target produces Localized Surface Plasmon Resonance (LSPR) resulting in a significant Raman signal amplification up to several orders of magnitude when excited by an external magnetic field. In-addition, the reported Raman enhancement factor is at $10^{9}$ has been solely due to the presence of the metal tip. Thus, TERS has demonstrated its success as a non-destructive method in determining the physical and chemical properties of materials at nanometer scale level that would find a wide array of applications in both material and biological sciences ${ }^{195,196}$. But, the fixed nature of these probes and the limitation offered by Raman cross-section due to the presence of single probe severely restricts its potential to monitor and sense the real-time molecular dynamics happening within the cell ${ }^{196}$. For instance, current approach to TERS for biosensing and cancer diagnostics requires the 
probe to be in constant contact or at least in the near vicinity of the biological environment. This factor severely restricts the availability of the critical information due to surface chemistry interactions within cellular microenvironment. To address this specific limitation, the use of targeted tags/receptors very specific to cells is now encouraged. The TERS probes can then not only sense the tags but in-turn can also report the cellular information. Since the tags used are specific in nature, and the inability to accommodate multiple probes within the same detection system, limits information sensed at the already limited and localized cross-section area. Moreover, it does not account for application in a complex cellular microenvironment wherein all the cellular functions are interdependent ${ }^{197}$. Thus, to precisely sense the biological events, the probes must necessarily have the inherit features and the ability to detect multiple biological elements such as DNA, RNA, proteins, amino acids, and lipids present within the cell at ultra-low concentrations without the need for labels or tags.

Furthermore, since the discovery of TERS phenomenon, a significant amount of effort has been devoted in exploring the in-vitro and in-vivo potential TERS for intracellular detection of proteins, integrins, DNA and RNA present in cells and tissues ${ }^{197,198}$. For instance, Singhal et al., investigated on the use of gold coated carbon nanotube AFM tip on intracellular molecules detection ${ }^{199}$. Similarly, Vitol et al., executed an in-situ intracellular sensing probes using gold decorated nanopipettes ${ }^{200}$. Although these methods allowed for precise targeting of cellular organelles, the need for complicated instrumentation and its inability to track live cell targeting due to lack of portability posed a fundamental problem. This is primarily due to the destructive nature of cell lysing due to tip cross-section. In-addition, it is often labor intensive, requiring large sample volumes and to go through an elaborate sample preparation technique. Although, current 
approach to TERS metals such as Au and Ag which offer high spatial confinement of Raman signal enhancement due to tip-sample gap, they are limited in its use and not an ideal candidate for realtime biosensing and cancer diagnostics applications. The high spatial resolution and the varying signal enhancement from the probes makes investigations for quantitative measurements at nanometer scale very challenging.

In-addition, the current approach to TERS is limited in its ability only to use single probe tip to interact with the target area only. This severely limits the Raman cross-section, as the emphasis is more focused towards tip spatial resolution and on tip - target gap level. A feasible approach to solve this issue and to overcome the limitation is by increasing the Raman cross-section area. This however was achieved using yamazoe et al., using gold nanoparticles grown over random boehmite nanoarray. Although this approach solved the limited Raman cross section challenge it lacked the desired sensitivity as it mimicked Surface Enhanced Raman Spectroscopy (SERS) rather than TERS ${ }^{201}$. Therefore, to address all the above said limitations, one viable technique is to make multiple TERS probes which are all portable and are also label-free so that they can be transported inside the targeted cellular microenvironment at the desired site so as to monitor and sense all the real-time events.

Here, we report the successful synthesis and implementation of portable quantum probes with atomic antennas to the specific desired site of interest for biological sensing applications. The quantum probes synthesized are developed both with and without multiple atomic scale edges so as to mimic conventional TERS. The quantum probe edges are in atomic scale and thus essentially act as antennas in amplifying Raman signals of individual components that make up the cellular signature. The multiple atomic antennas present in the quantum probes are focused to enhance 
crucial intracellular molecules such as DNA, RNA and proteins to higher enhancement efficiency. These quantum probes also demonstrated Single Molecule (SM) sensitivity of known Raman probe molecules Crystal Violet (CV), Rhodamine 6G (R6G) at $10^{12}$ enhancement factor when evaluated at pico and femtomolar concentrations. Furthermore, to explicitly showcase its biosensing ability, nanomolar concentration of Bovine Serum Albumin (BSA) was evaluated. We also demonstrated the feasibility of using quantum probes for cancer cell detection (HeLa and MDAMB-231) and distinct differentiation from healthy fibroblast cells. This was achieved using a time-based analysis between 6 to 24 hours duration wherein live monitoring of cellular behaviors, cell apoptosis and proliferation could be identified. To the best of our knowledge, this is the first reporting of a successful non-TERS approach undertaken for detection of live cancer cell and specific differentiation made using quantum probes with atomic antennas.

\subsection{Materials and Methods:}

\subsubsection{Materials:}

Commercially available nickel 200 sheets (0.125-inch-thick) having 99.9\% nickel sourced from (onlinemetals.com, USA) was cut into 1-inch square pieces. The cut sample surfaces were then mechanical polished using SiC grit sandpaper (200 to 1200) and later ultrasonically cleaned in $100 \%$ acetone and ethanol for a 15-minute duration each to remove all the embedded particles inclusions derived due to the polishing process. The polished sample surface was later washed with de-ionized (DI) water and consequently air dried for further experimentation. 


\subsubsection{Synthesis of quantum probes:}

The generation of quantum TERS probes was achieved using Clark-MXR Impulse series pulsed Ybdoped fiber amplified femtosecond laser to ionize nickel substrate. This ultra-short pulsed laser induces multiphoton ionization mechanism to form quantum probes by maintaining an average laser power of 16 Watts, with laser pulse repetition rate $(4,8,12,26 \mathrm{MHz})$ maintaining central wavelength of $1030 \mathrm{~nm}$ and holding a constant pulse width of $214 \mathrm{fs}$. To create an even distribution of quantum probes that is viable for SERS enhancement, a piezo-driven raster system was used to ionize the $\mathrm{Ni}$ substrate in a $300 \times 300$ point array with $0.25 \mathrm{~mm}$ spacing designed by Ez-CAD software. The complete ionization process was maintained in an inert $\mathrm{N}_{2}$ gas atmosphere where the gas was injected into the laser material interaction zone through several individual nozzles which evenly surrounds the ionization zone. The $\mathrm{N}_{2}$ gas introduced into the ionization zone was maintained at 1 bar pressure.

\subsubsection{Physicochemical characterization of quantum probes:}

The quantum probes were imaged using a field emission and high-resolution scanning electron microscope (Quanta - FESEM and HRSEM). The HRSEM/EDX attachment from Oxford Instruments was used to analyze elemental composition of the synthesized probes. The physical morphology of the probes was imaged using a JEOL 2100 High Resolution Transmission Electron Microscope on copper mesh grids. The size distribution was tabulated using ImageJ software. The X-ray diffraction (XRD) crystallographic analysis was performed using Phillips diffractometer. A $2 \theta$

scanning range from 10 to $90^{\circ}$ was used to acquire the relevant peaks associated with nickel and nickel oxide quantum probes. Subsequently Reitveld analysis was also carried out on the acquired spectrum. Thereafter, to acquire the Raman spectra of both quantum probes and chemical 
molecules, a Bruker SENTERRA Raman confocal microscope with 50X magnifying lens at 785nm obtained at $5 \mathrm{~mW}$ power respectively was deployed. To achieve a consistent significant signal a 60s integration time was employed so as to maintain a consistent signal response, 3 co-additions were acquired for each Raman spectrum in this study.

\subsubsection{Raman Enhancement factor calculation:}

The Raman enhancement factor (EF) for atomic antennas was analyzed experimentally using the following Raman EF equation present in most accepted literature $142,171,202$.

$$
E F=\frac{I_{\text {Atomic anternna }} / N_{\text {Surface }}}{I_{\text {substrate }} / N_{\text {bulk }}}
$$

$I_{\text {Atomic antenna }}$ represents the Raman intensity on nickel nanonetwork, $I_{\text {substrate }}$ represents the Raman intensity on nickel substrate, $\mathrm{N}_{\text {bulk }}$ represents the number of molecules present under the confocal volume and $\mathrm{N}_{\text {surface }}$ represents the number of molecules responsible for the response. A $10 \mu \mathrm{l}$ volume of dye was added on to the quantum probes and nickel substrate for evaluating raman response. From Figure 5-4, the values noted are $I_{\text {Atomic Antennas }}=4955$ and $I_{\text {substrate }}=131$ at $1620 \mathrm{~cm}^{-}$ 1. The CV molecule has strong vibrational modes at $1620 \mathrm{~cm}^{-1}$ wavenumber and hence was used in EF calculations ${ }^{203}$. The following equation was used to calculate $\mathrm{N}_{\text {bulk. }}$.

$$
N_{\text {bulk }}=\pi r^{2} h c N_{A} \quad \text { Eq.2 }
$$

Where $\mathrm{N}_{\mathrm{A}}$ is Avogardo constant, $\mathrm{r}$ represents the Raman laser spot radius (1.5 $\left.\mu \mathrm{m}\right), \mathrm{h}$ is the half the depth of Field (DOF) of a 50x magnified laser used in the study $(2 \mu \mathrm{m})$, c represents crystal violet (CV) appropriate concentration $\left(1 \times 10^{-12}, 1 \times 10^{-15}\right) \mathrm{M}$ was dropped on the substrate. $\mathrm{N}_{\text {bulk }}$ was calculated using equation 2 and to calculate $\mathrm{N}_{\text {surface, }}$ surface area and $\mathrm{CV}$ absorption on quantum 
probes are required and necessary. Some conservative estimates of quantum probes were made in calculating $\mathrm{N}_{\text {surface }}$ from the work of Chinnakkannu Vijayakumar et al., and Powell J.A. et al., as a very similar ion plume formation mechanism is employed to create Nickel and silicon nanonetworks ${ }^{24,61}$. The density of the quantum probes with atomic antennas was assumed to be identical to the bulk nickel $\rho=8.90 \mathrm{~g} / \mathrm{cm}^{3}$ and BET surface area as $24 \mathrm{~m}^{2} / \mathrm{g}$ was obtained from literature ${ }^{204}$. The TERS experiments was carried out immediately after CV dye application upon atomic antennas and nickel substrates. To approximate the number of molecules that contribute to Raman enhancement from the quantum probes, we need to consider the number of dye molecules that adsorb onto the surface of quantum probes. Since, it is not practically feasible to weigh cubic micrometer volumes, a similarity relationship was established. For both $\mathrm{N}_{\text {bulk }}$ and $\mathrm{N}_{\text {surface }}$ we have assumed only the molecules present within the interaction volume of the Raman laser that directly contribute to Raman enhancement. $N_{\text {surface }}$ was calculated using the equation 3 ,

$$
N_{\text {surface }}=S A_{\text {eff }}\left(C_{\text {ads }} N_{A} \frac{1000 L}{1 m^{3}}\right)^{2 / 3} \quad \text { Eq.3 }
$$

Where $S_{\text {eff }}$ was the effective surface area of quantum probes within Raman laser interaction volume, $C_{\text {ads }}$ was the surface adsorption of the dye molecule and $\mathrm{N}_{\mathrm{A}}$ was Avagardo's constant. Finally, the enhancement factor was calculated using equation 1. Similarly, the enhancement factor of R6G was also calculated by substituting the $\mathrm{CV}$ values with R6G from Powell J.A. et al., ${ }^{24}$ 


\subsubsection{Optical characterization of quantum probes:}

A colloidal suspension of the synthesized theranosomes was generated using Phosphate Buffer Solution (PBS) and Hitachi UV-3100 UV-vis-NIR spectrophotometer was used to analyze UV-visNIR absorption level and also the reflection spectrums of both the synthesized quantum probes.

\subsubsection{Preparation of Raman probe molecules (Crystal violet (CV) and Rhodamine 6G (R6G)):}

Selection of both CV and R6G dyes was due to its large Raman cross-section offered, and moreover it was quite used for testing raman scattering effect. A stock solution of CV and R6G was prepared from an anhydrous powder purchased from Sigma -Aldrich with an initial concentration level of 1 $\mathrm{M}$ and thereafter subsequently diluted to $1 \times 10^{-12}$ and $1 \times 10^{-15} \mathrm{M}$ concentration levels using ultrapure water for experimentation.

The acquisition time was kept constant at 30 s and 3 acquisitions made for of each spectrum to ensure reproducibility and uniformity of signals. $10 \mu \mathrm{l}$ of the specified concentration of CV and R6G solution was applied to quantum probes just prior to the collection of Raman spectra.

\subsubsection{In-vitro cell culture for Transmission Electron Microscopy:}

HeLa, human cervical cancer cell line, was obtained from ATCC (American type culture collection, ATCC No. CCl-2) and were cultured in RPMI-1640 medium with phenol red containing $10 \%$ heat inactivated fetal bovine serum and $1 \%$ penicillin-streptomycin antibiotics at $37^{\circ} \mathrm{C}$ in a $5 \% \mathrm{CO}_{2}$ atmosphere. NIH3T3 fibroblast cells and MDAMB-231 were also obtained from ATCC and were cultured in DMEM medium containing $10 \%$ fetal bovine serum with $1 \%$ penicillin-streptomycin antibiotics at $37^{\circ} \mathrm{C}$ in $5 \% \mathrm{CO}_{2}$. The quantum probes were washed with alcohol and $\mathrm{DI}$ water and then kept under UV light for a duration of 15 minutes. The substrates were then placed in separate petri-dishes with HeLa, MDAMB-231 and NIH3T3 cells were seeded individually to attain a density 
level of $10^{5} \mathrm{cell} / \mathrm{ml}$, volume totaling to $4 \mathrm{ml}$ by quantity per dish. The petri dishes were then placed in an incubator for 24 and 48 Hours.

After the set incubation period, the samples were fixed for TEM imaging in $2 \%$ glutraldehyde in $0.1 \mathrm{M}$ sodium cacodylate buffer and stored at $4^{\circ} \mathrm{C}$ until sectioning. The cells were then rinsed in buffer and fixed in $1 \%$ osmium tetroxide in buffer, dehydrated in graded ethanol series followed by propylene oxide and later embedded in EMBed812 resin. Cells were then stained with uranyl acetate and lead citrate. Then RMC MT6000 ultramicrotome was used to cut $100 \mathrm{~nm}$ thick sections and later viewed in an FEI Tecnai 20 TEM. TEM preparation and imaging were performed at Nanoscale Biomedical Imaging Facility for Sick Children Research Institute, Toronto, Canada. Multiple separate images were chosen for each condition to further conduct the quantitative analysis for measuring cell analysis using ImageJ software.

\subsubsection{Quantum probe in-vitro assessment:}

The Raman properties of the samples were examined by using a portable handheld Raman spectrometer (B\&W Tek Inc.) using a 785-nm semiconductor laser as the excitation source. The laser power at the sample position was $150 \mathrm{~mJ}$ and the accumulation time was $60 \mathrm{~s}$; the scattered radiation was collected by a 40X objective lens with a numerical aperture (NA) of 0.65 .

\subsubsection{Statistics:}

All experiments were carried out in triplicates and all data points collected were set to standard mean with \pm standard error unless otherwise mentioned. The error bars included also indicate the standard deviation. On the whole an overall 95\% confidence interval for the mean was achieved. 


\subsection{RESULTS AND DISCUSSION:}

\subsubsection{Synthesis of quantum probe by ultra-short ionization mechanism of solid nickel substrate:}

The synthesis of portable Quantum probes over nickel substrate by ultra-short pulsed laser interaction has been depicted in schematic figure 5-1. To synthesize intertwined atomic scaled antennas, a computer controlled scanner was employed which allowed for precise two dimensional (2D) movement of ultra-short laser beam over nickel substrate in $X-Y$ direction. The quantum probes with atomic antenna generation by ultra-short laser pulses in the presence of inert nitrogen atmosphere was a non-catalytic, non-linear gas dynamic process which involved a specific sequence of actions: surface energy absorption of laser pulses, plasma plume ignition \& ionization, rapid condensation and ejection of species to atmosphere. In ionization process, ultrashort laser pulses with nanosecond pulse to pulse seperation time (ns) with microjoule $(\mu \mathrm{J})$ energy was focused onto the nickel surface, resulting in ionized species formation within the interacting plume which later cools to form atomic scale tips in quantum probes. During ultrashort pulsed laser irradiation, the energy was delivered into the material in a short time scale far exceeding the material threshold to initiate nanoscale ablation. After such sucessive laser pulses, the nanoscale particle further ionizes to form nickel ions. The expanding ion plume carries the ionized material further away from the surface. The expanding ionic species thereafter condense to form sub-nanometre scale particles. Here, the energy deposited by ultrashort laser pulses exceeds inter-atomic binding energy and the atom under excitation becomes free from their bonds to interact further with the incoming ultra-short laser pulses. This initiates non-catalytic kinectic reaction within the explanding plume to stay in a gaseous-ionic state encompassing noninteracting atoms ejected from the interacting material surface to further interact with the 
oncoming laser pulses in an ambient atmosphere. Since laser pulse interaction time is in the orders of magnitude shorter than the material heat diffusion time, it induces superheated ion formation reaction creating ionised species which totally transfigures the material crystalline structure. This swift transformation from initial cold solidified state to an ionic state of substrate material when under excitation causes a time-dependent ionization and material recombination. The final ejection of ionised spiecs from the plasma plume outwards forces the superheated ions to cool down as it propels away from interacting surface recombining with oxygen present in ambient atmosphere condensing to form intertwined quantum probes. The growth rate of each and every individual probes within the self-assembled quantum probe varies with lattice plane orientations and the temperature gradient, thereby enabling effective control over both probe morphology and its size. The morphology of the synthesized quantum probe was analysed and was observed to have a wider size distribiution of probes within the observed structure from graphs in figure 5-1. The formation mechanism showcased here is unique to the laser-ion plume formation as demonstrated in our previous works $76,205,206$. 


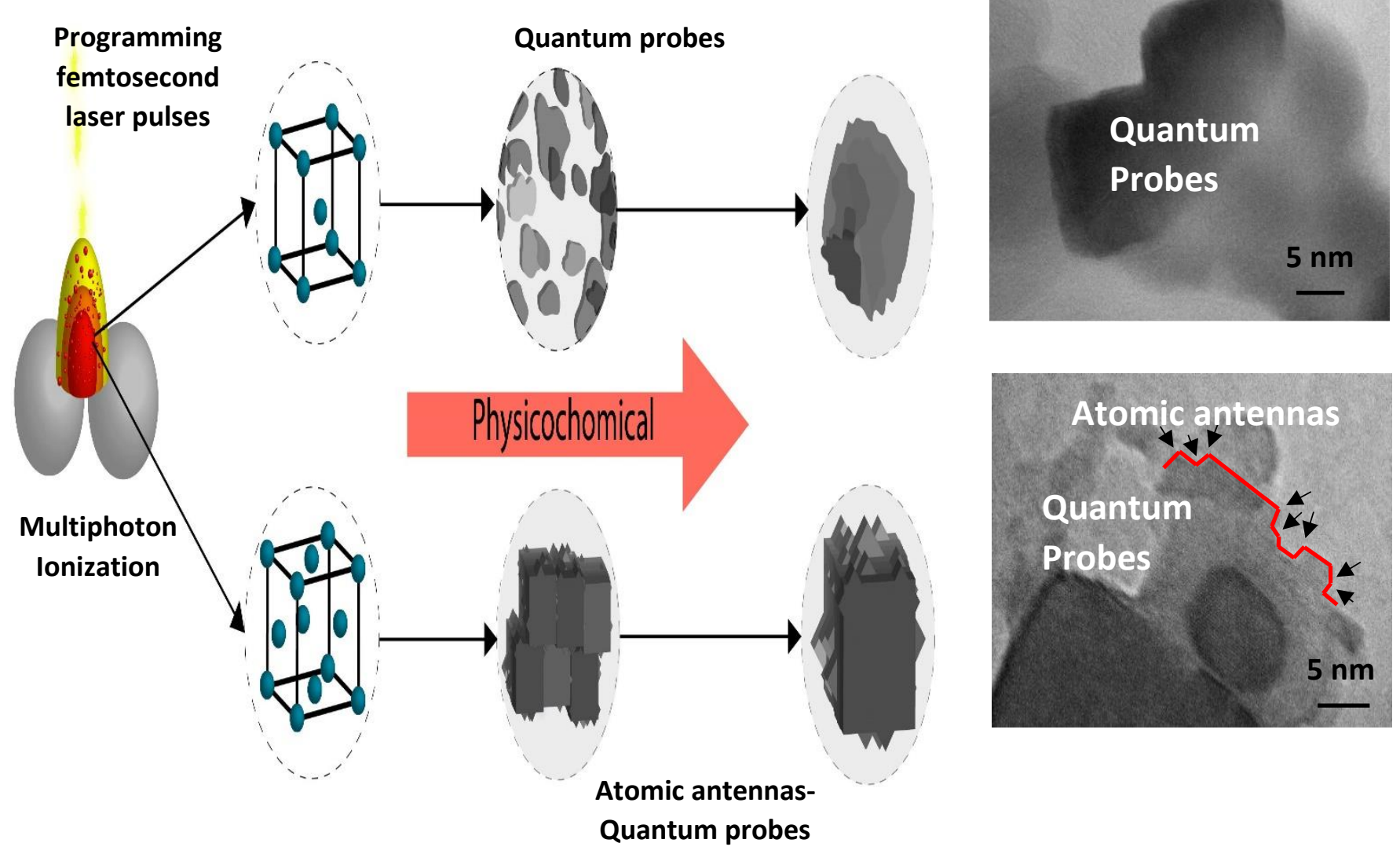

Figure 5-1: Multiphoton ionization mechanism to generate quantum probes with atomic scale antennas 

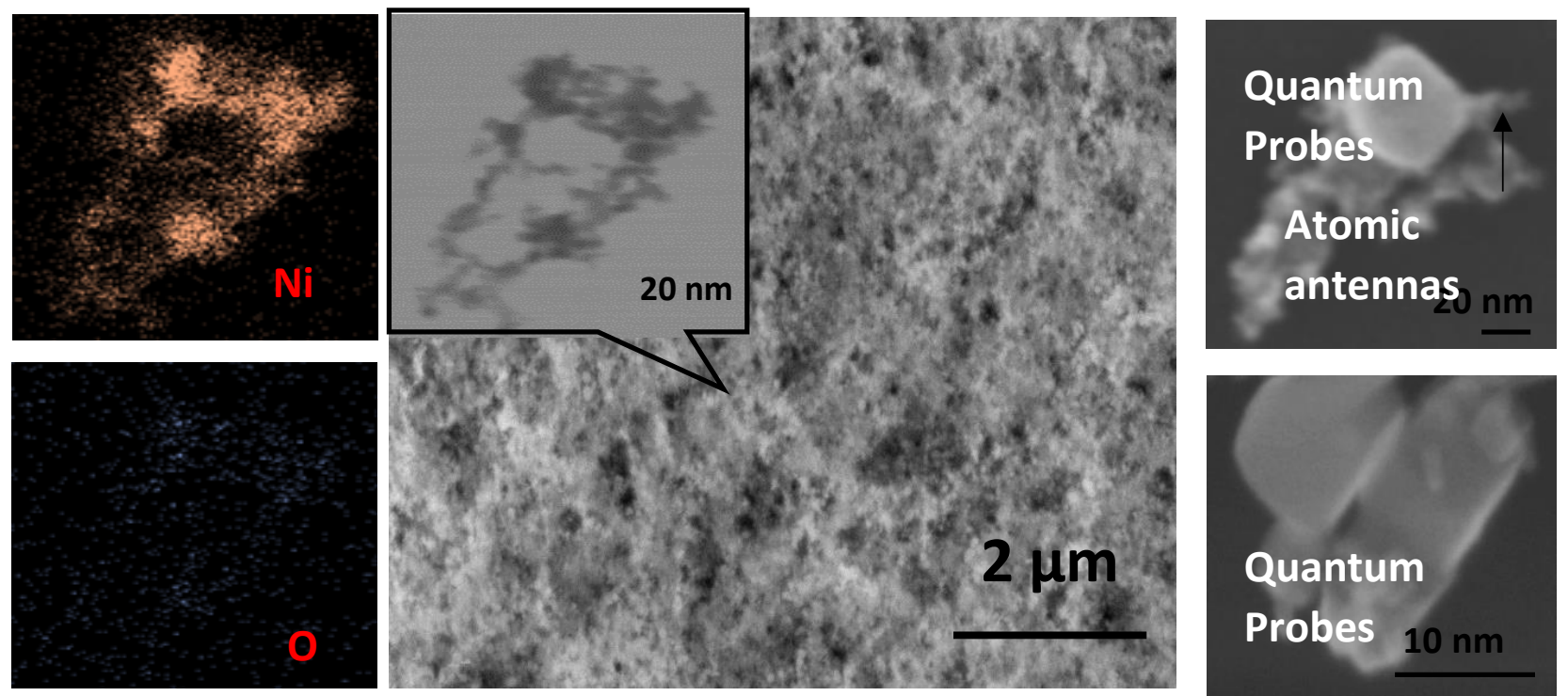

Histogram of Particle size $(\mathrm{nm})$

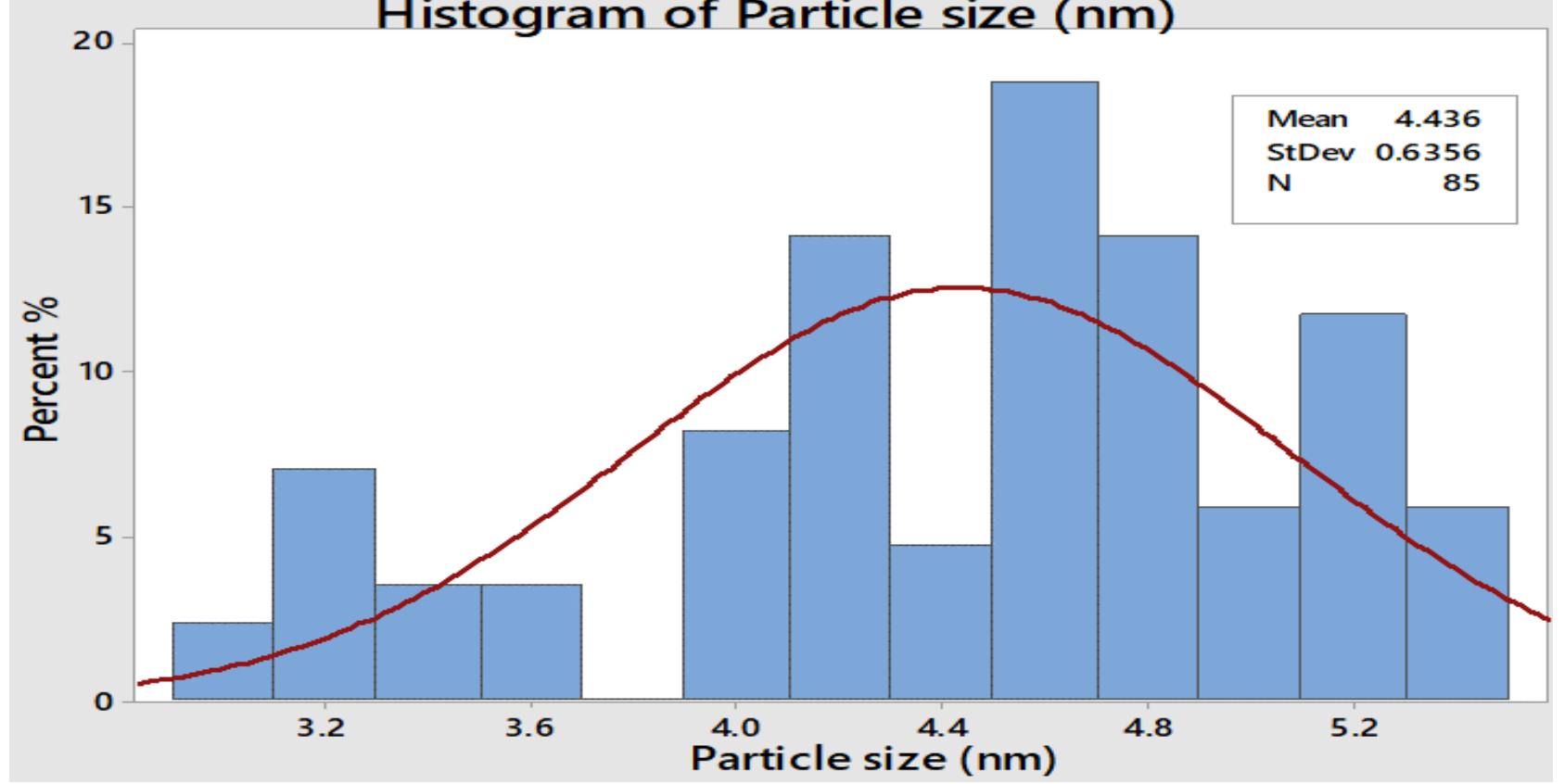

Figure 5-2: Portable quantum probes with atomic antennas morphological and size distribution

We observed Raman bands in the spectral region above 400 and within $1500 \mathrm{~cm}^{-1}$. The Raman peaks noted in Figure 3 are primarily vibration modes due to the first and second order Raman scattering by phonons, whereas the band above $1200 \mathrm{~cm}^{-1}$ originates due to scattering by two magnon modes. In general, magnons are observed in all magnetically materials due to the electron spin 

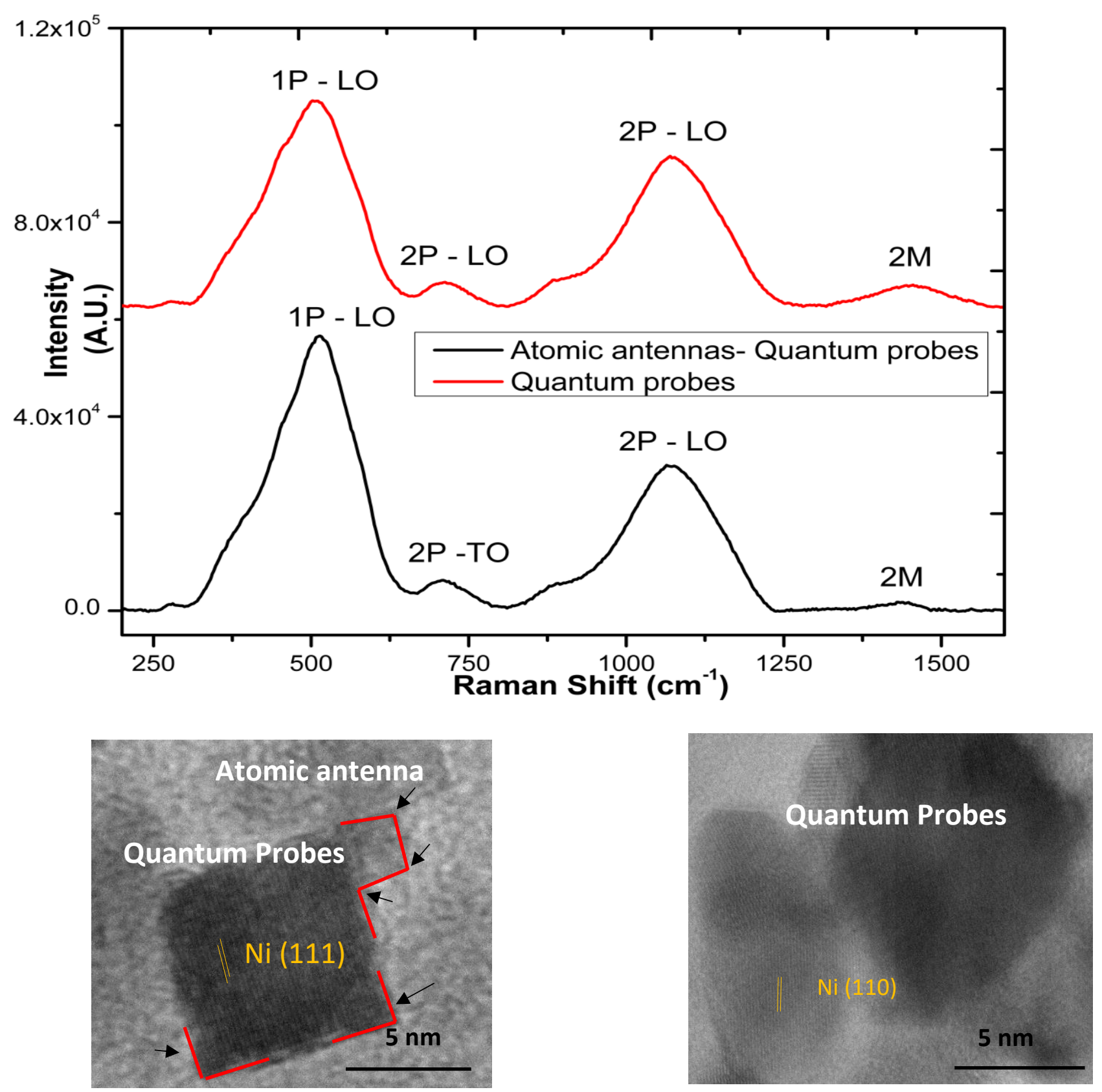

Figure 5-3: Raman spectrum of portable quantum probes with atomic antennas

waves excitations. But, these inelastic scattering observed with magnons can also occur in magnetic materials due to spin-orbit coupling ${ }^{97}$. The strong Raman band observed in figure 3 at $\sim 510 \mathrm{~cm}^{-1}$ can be assigned to the first-order one phonon (1P) LO mode. The Raman band observed near $500 \mathrm{~cm}^{-1}$ could also be of magnetic origin. In this present work, the Raman bands 
positioned at $\sim 510 \mathrm{~cm}^{-1}$ can be directly related to atomic scale particle as particle size influences magnetic behavior ${ }^{98}$. In-addition, there was complete absence of the first order TO mode in both conditions but second order 2 TO mode was found at $\sim 711 \mathrm{~cm}^{-1}$. We therefore assigned the $\sim 1069$ $\mathrm{cm}^{-1}$ to the second order LO phonon modes. Moreover, the two magnon modes $(2 \mathrm{M})$ at $\sim 1400$ $\mathrm{cm}^{-1}$ intensity was observed at both conditions. From the literature and previous studies, it was presumed that $2 \mathrm{M}$ vibrations seen here are primarily due to antiferromagnetic super exchange interaction in nickel ions in the linear atomic chain $\mathrm{Ni}^{2+}-\mathrm{O}^{2-}-\mathrm{Ni}^{2+}$.

\subsubsection{Raman scattering effect of Crystal Violet (CV) on quantum probes:}

To determine the Raman scattering efficiency of quantum probes Crystal Violet(CV) and Rhodamine $6 \mathrm{G}(\mathrm{R} 6 \mathrm{G})$ was evaluated at pico $\left(10^{-12}\right)$ and femtomolar $\left(10^{-15}\right)$ concentrations. CV was a common cationic dye with a symmetric molecular structure. The native bulk nickel substrate had no response to the presence of the CV dye irrespective of the concentration level. However, the dye-coated quantum probes display the characteristic peaks for the associated dye at both $10^{-12}$ and $10^{-15} \mathrm{M}$ concentrations as shown in inset of figure $5-3$. The spectrum had relative shifts in peak intensity and positions owing to strong surface reactions between CV dye molecule sand atomic antennas present within the quantum probes. This translates to selective dye enhancement which were not observed with other TERS. In-addition, the vibrations of the central carbon $\left(\mathrm{C}+\right.$ ) phenyl bonds are assigned from $100-400 \mathrm{~cm}^{-1}$ and phenyl vibrations are above 400 $\mathrm{cm}^{-1}$ from Yamada et al., ${ }^{160}$. 

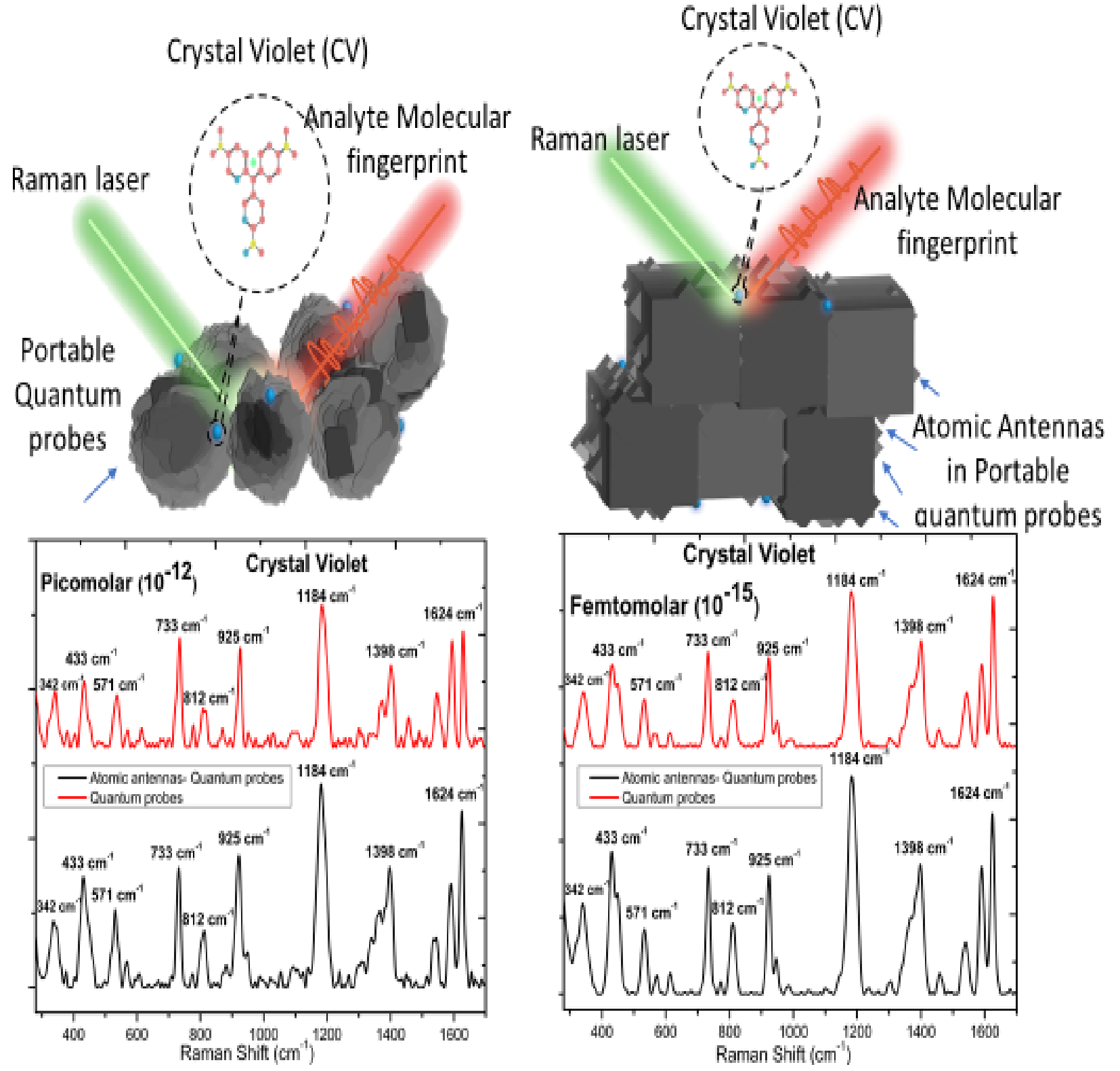

Figure 5-4A: Quantum probe interaction at picomolar, femtomolar concentration of CV

These observed results indicate the dependency of the characteristic Raman intensity peaks of $\mathrm{CV}$ on quantum probes. Both picomolar and femtomolar concentrations of CV dye induced Raman response up to $1700 \mathrm{~cm}^{-1}$. Figure 5-4A highlights the quantum probes response spectra of CV wherein the peaks of CV at $570 \mathrm{~cm}^{-1}, 925 \mathrm{~cm}^{-1}, 1624 \mathrm{~cm}^{-1}$ corresponds to ring skeletal vibration 
of radial orientation, in-plane ring $\mathrm{C}-\mathrm{H}$ vibration, $\mathrm{C}-\mathrm{C}$ stretch vibration respectively ${ }^{207}$. Lombardi and Birke have established a comprehensive theory emphasizing that an enhancement factor above $10^{4}$ cannot solely result from charge transfer mechanism. The enhancement factor achieved here is in the order of $10^{12}$. The strong response observed here is thus solely due to "hot spot" enhancement ${ }^{142}$.

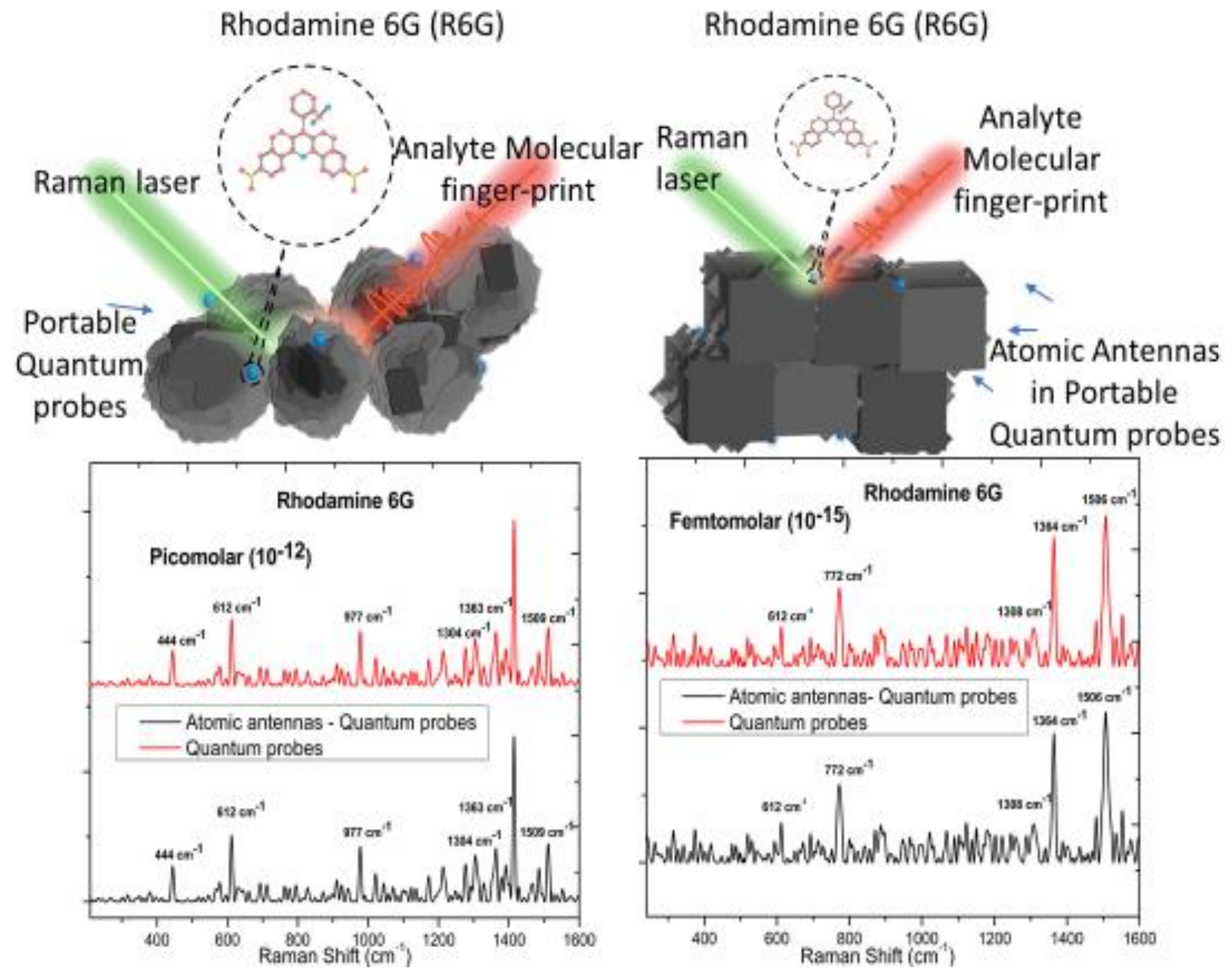

Figure 5-4B: Quantum probe interaction at picomolar, femtomolar concentration of R6G

5.3.3 Raman scattering effect of Rhodamine 6G (R6G) on quantum probes:

The vibrational fingerprint of R6G exhibits prominent Raman response with peaks at $612 \mathrm{~cm}^{-1}$, $772 \mathrm{~cm}^{-1}, 1509 \mathrm{~cm}^{-1}$ corresponding to in-plane C-C ring vibration, out-of-plane C-H bending, and 
aromatic ring vibration respectively ${ }^{24}$. In-addition, the intensity distribution is uniform and fits in a gaussian curve, demonstrating the obtained spectra is from a single molecule of R6G. The peak centered at $1620 \mathrm{~cm}^{-1}$ for $\mathrm{CV}$ and $612 \mathrm{~cm}^{-1}$ for R6G was used to calculate enhancement factor since it does not have any interference from the Raman signal of quantum probes. The signal enhancement achieved by quantum probes resemble electromagnetic hotspots resulting in an ultrasensitive Raman enhancement wherein multiple atomic scale antenna enhance signal intensity by quantum edge and size effects. This phenomenon is proved by the increased number of peaks in CV and R6G Raman spectrum at ultra-low concentration levels. The above results conclude that the quantum structure with atomic antennas can sense probe molecules to femtomolar concentrations. The enhancement factor achieved is in the order of $3.1 \times 10^{12}$ for femtomolar concentration of the $\mathrm{CV}$ molecule and $3.58 \times 10^{12}$ for femtomolar concentration of R6G molecule. It can also be inferred from figure 5-4(A, B) that the enhancement of Raman signal does not saturate at femtomolar concentration irrespective of quantum probe type suggesting that the quantum probes function is very similar to the hotspots clustering effect resulting in an ultrasensitive enhancement which has the potential to sense close to zepto molar analyte concentrations.

\subsubsection{Raman scattering effect of Bovine Serum Albumin (BSA) biomolecules and label-free} cell adhesion and quantum probe uptake in cells:

Proteins and other complex biomolecules consist of different amino acids. Proteins with different structures hold different biological functions. BSA consist of 19 different amino acids ${ }^{208}$. The Raman fingerprint region of BSA is between 400 to $1600 \mathrm{~cm}^{-1}$ provides both structural and 
functional group present in the microenvironment. As shown in the above figure 5-5 Raman signals of BSA can be easily observed with quantum probes at nanomolar concentrations.
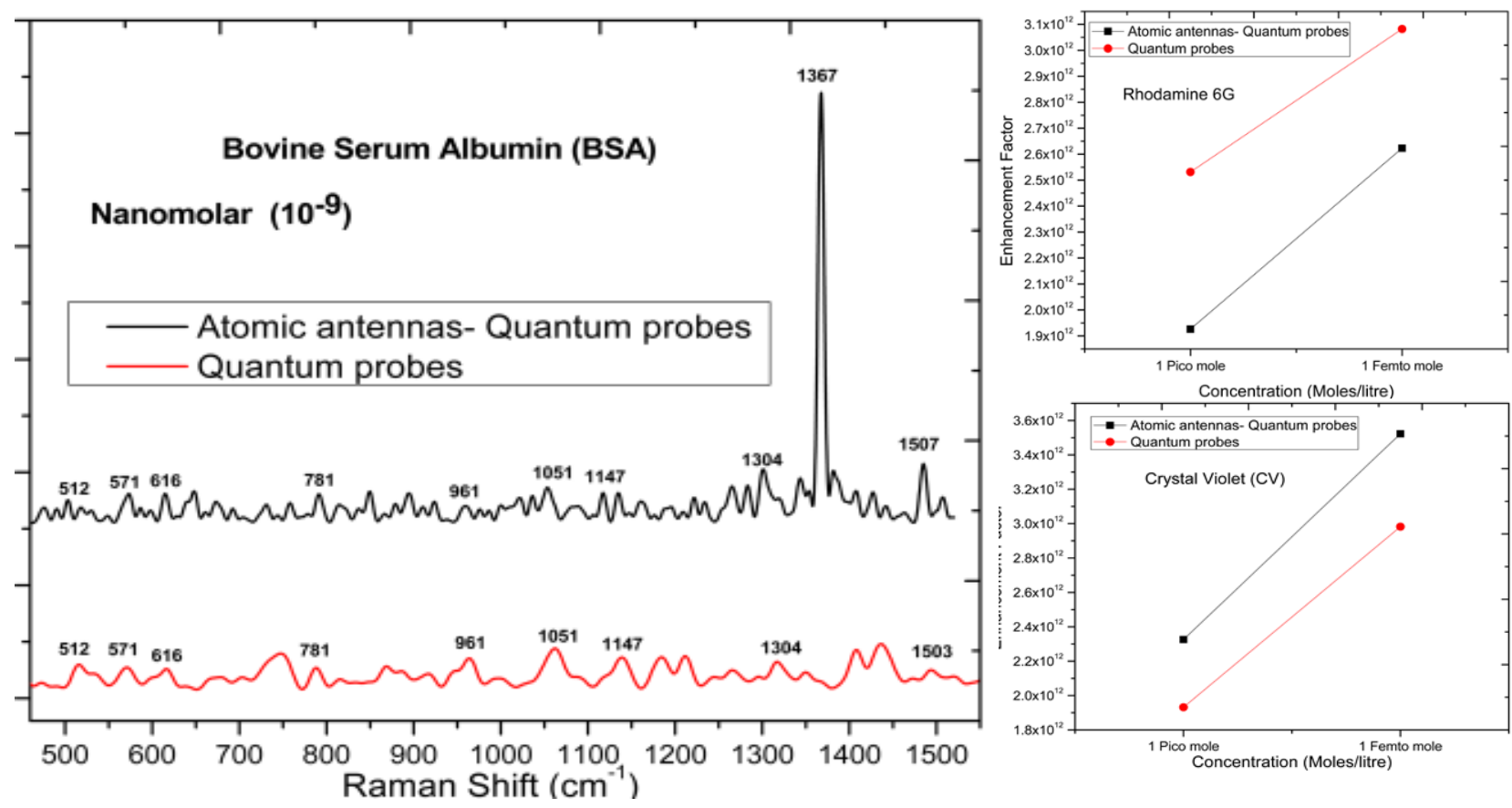

Figure 5-5: Quantum probes interaction at nanomolar concentration of BSA along with enhancement factor relationship for crystal violet and rhodamine $6 G$

Furthermore, the presence of $512 \mathrm{~cm}^{-1}$ on both sharp and smooth TERS probes is associated with S-S mode in BSA. In-addition, the observance of this S-S mode is also used as an indicator to showcase the intact nature of the molecule during experimentation. The observance of this S-S bond also suggests the TERS signal enhancement is solely due to electromagnetic enhancement from the literature ${ }^{208}$. The peak observed at $966 \mathrm{~cm}^{-1}$ is associated with symmetric stretching vibrations of carboxylic acid groups. The TEM images reveal that both cancerous and fibroblast cells could adhere and proliferate in the presence of quantum probes. In general, at nanobiointerface level, saturation takes place when excessive nanoparticles surround any cellular environment ${ }^{209}$. This in turn is capable to induce irreversible modifications within the cellular environment such as cell binding and internalization of nanoparticles. 

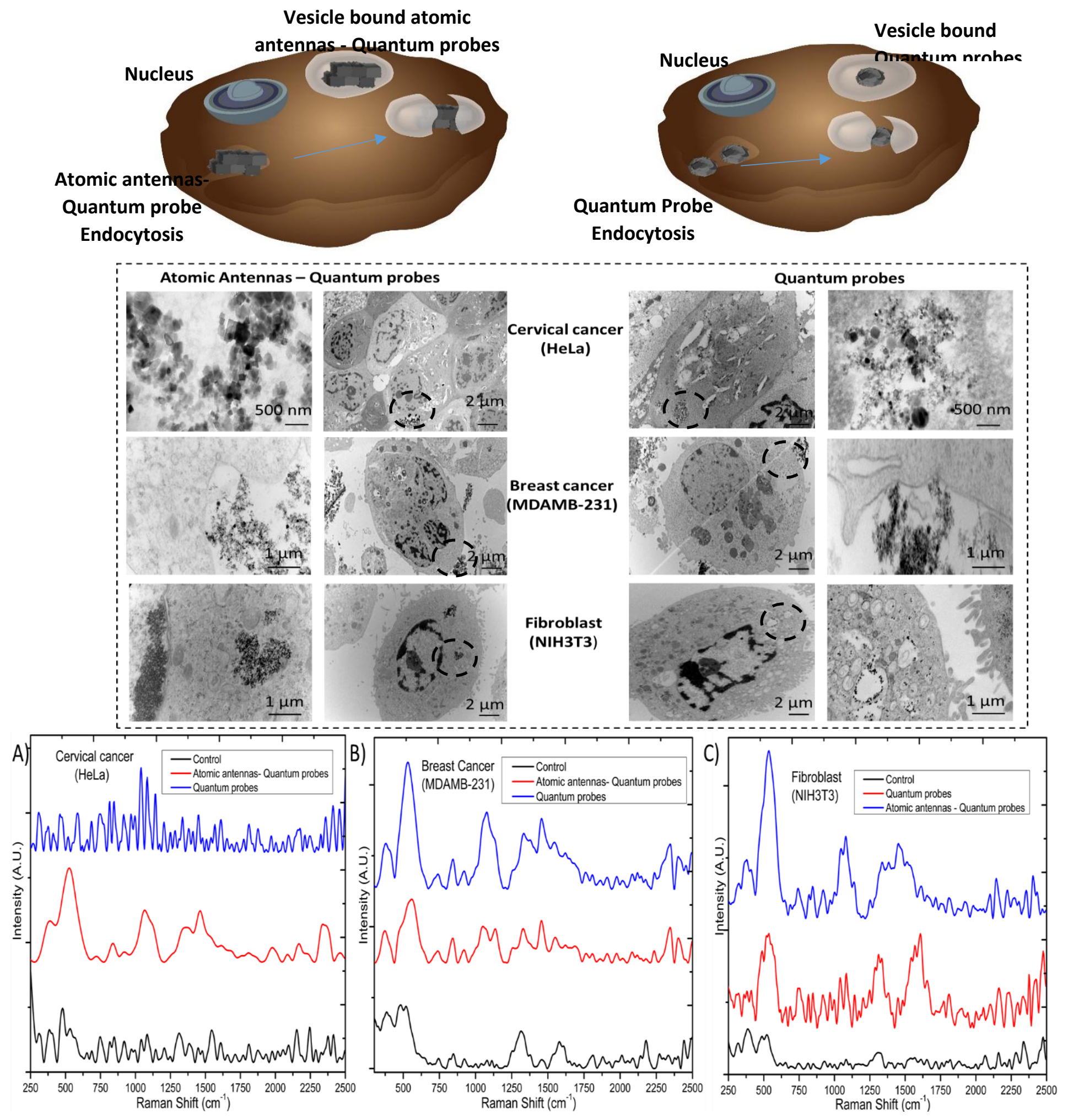

Figure 5-6: Quantum probe uptake in cells validated through TEM after 24 hours incubation and its corresponding Raman response A) HeLa B) Breast cancer C) Fibroblast cells 
Such nanoparticles tend to localize in certain intracellular organelles and thereby start to effect a change to specific cell areas ${ }^{210}$. The path that these nanoparticles travel inside the cell is primarily mediated through passive endocytosis and it is dependent on nanoparticle chemistry, size and or a combination of both ${ }^{211}$. Similarly, the cellular uptake of the quantum probes is predominately through passive endocytosis. Quantum probes after entering the cancer cells localized in endosomes, then mediate to lysosomes, cytoplasm and then mitochondria. These were verified using TEM images and further illustrated in figure 5-6. Since, endocytosis is a dynamic process, it involves the following steps i) binding of the quantum probes to cell surface and ii) internalization of quantum probes into cells. Nanoparticles adsorb various small molecules in biological system and these adsorbed molecules play a vital role in cell signaling and cell physiology ${ }^{212}$.

\subsubsection{In-vitro raman response to portable quantum probes for cancer detection using protein signature:}

Having demonstrated the presence of portable quantum probes inside the cells in the previous section, it is now possible to monitor quantum probe effect in cells, their proliferation and other functional changes to interacting cells in response to quantum probes. The presence of one phonon and two phonon longitudinal optical mode associated with quantum probes confirms the internalization of portable probes within the interacting cells. This is also confirmed by the quantum probes inside the nuclear envelope as seen from TEM observations illustrated in figure

5-6. Furthermore, the Raman response of both cancerous and non-cancerous cells clearly suggests difference in both peak position and intensity thereby not only confirming but essentially acting 

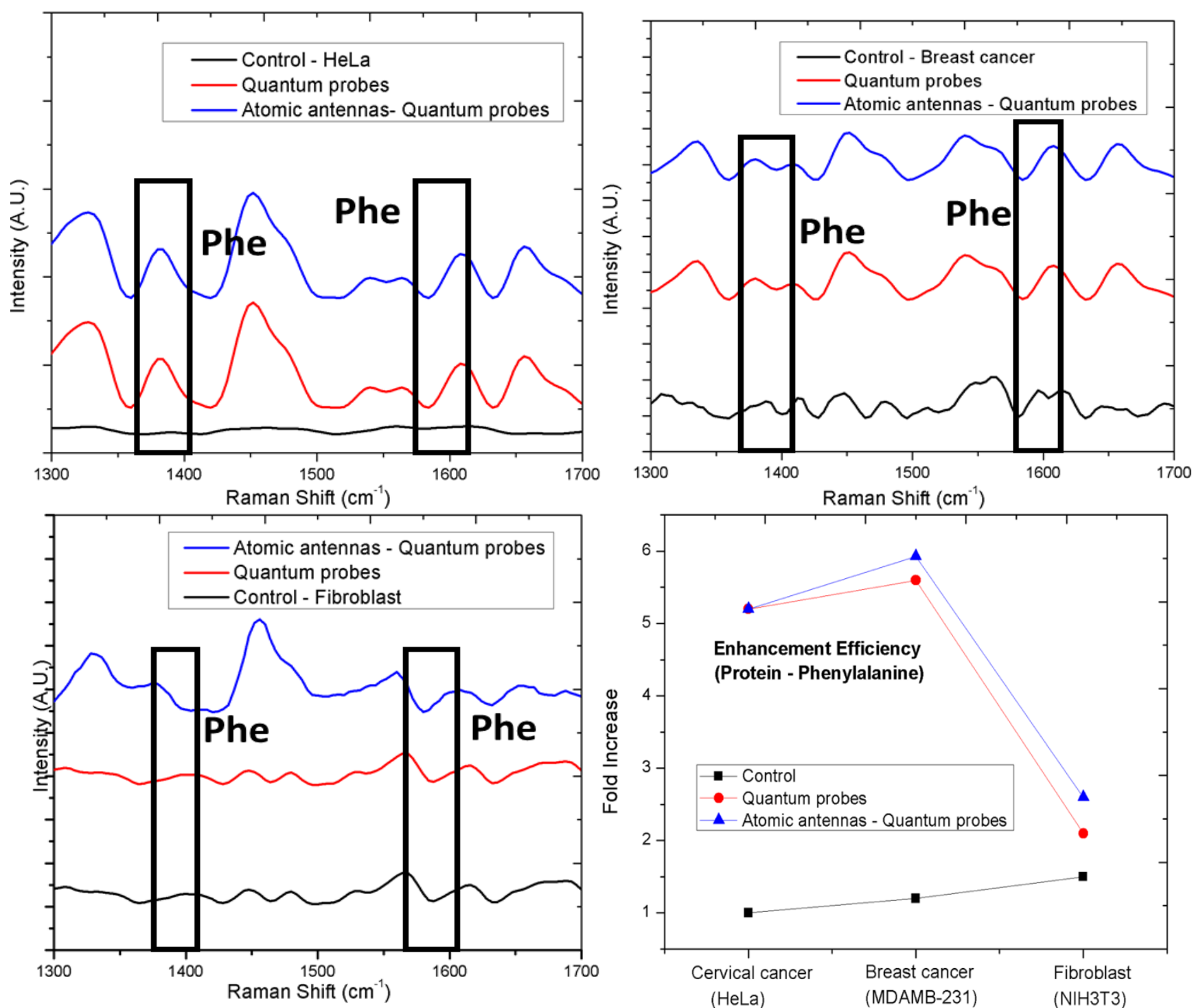

HeLa - cervical caner
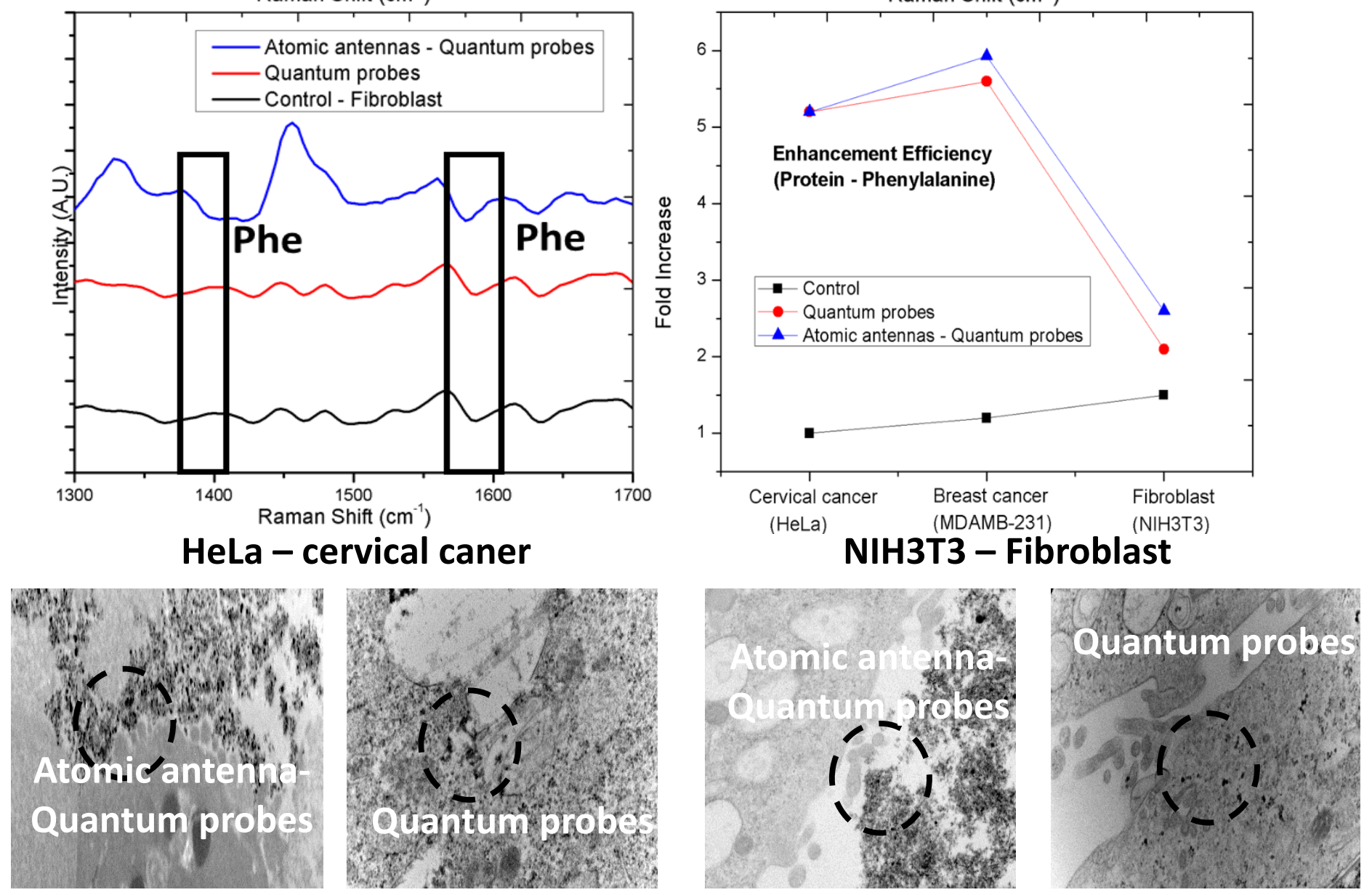

Figure 5-7: Detection of intracellular protein of cells in the presence of quantum probes. The peak positions at $1397 \mathrm{~cm}-1$ in combination with $1584 \mathrm{~cm}-1$ is identified as phenylalanine and tryptophan. The representative TEM images of HeLa and Fibroblast cells are showcased to verify quantum probe uptake in them. The location these probes is identified using black circular outline in TEM images 
as a unique identification signature. A visual comparison of the spectral group shows significant differences between the cancerous and fibroblast cells. Although the existence of certain peaks is common to all the three cells, they exhibit considerable difference in growth pattern and vibrational modes of molecules when present within the cells. The exposure of phenylalanine ring results in increased peak intensity in the Raman spectrum. Therefore, an increase in Raman intensity of phenylalanine signals is a definitive indicative of increased protein saturation. Furthermore, the increased protein content is a unique signature marker for identifying cancer cells as they are more aggressive in proliferating when compared with fibroblast cells. For instance, the absence or drastic decrease in proteins chiefly phenylalanine $\left(1584 \mathrm{~cm}^{-1}\right)$ when in combination with tryptophan peaks $\left(1397 \mathrm{~cm}^{-1}\right)$ proteins can also be used as indicators of cell type. The Raman response of both quantum probes is least on fibroblast when compared with those of cancer cells. The native control surface since it is not Raman active in nature did not show any considerable Raman response when compared with both quantum probes.

\subsubsection{Cancer cell differentiation using Raman response from portable quantum probes:}

Based on the Raman Spectra, it is possible to distinguish growth patterns as well as cellular origin and metabolism. The intensity of peak position $810 \mathrm{~cm}^{-1}$ is considered when analyzing RNA. The peak position $1066 \mathrm{~cm}^{-1}$ associated with O-P-O stretch is typical for DNA structure. The observation of these Raman peaks in general correspond to nucleic acids (DNA and RNA) are observed with high intensity suggest that these quantum probes could also sense the nuclear membrane without disruption to nuclear membrane. This can be verified by the selective particle 

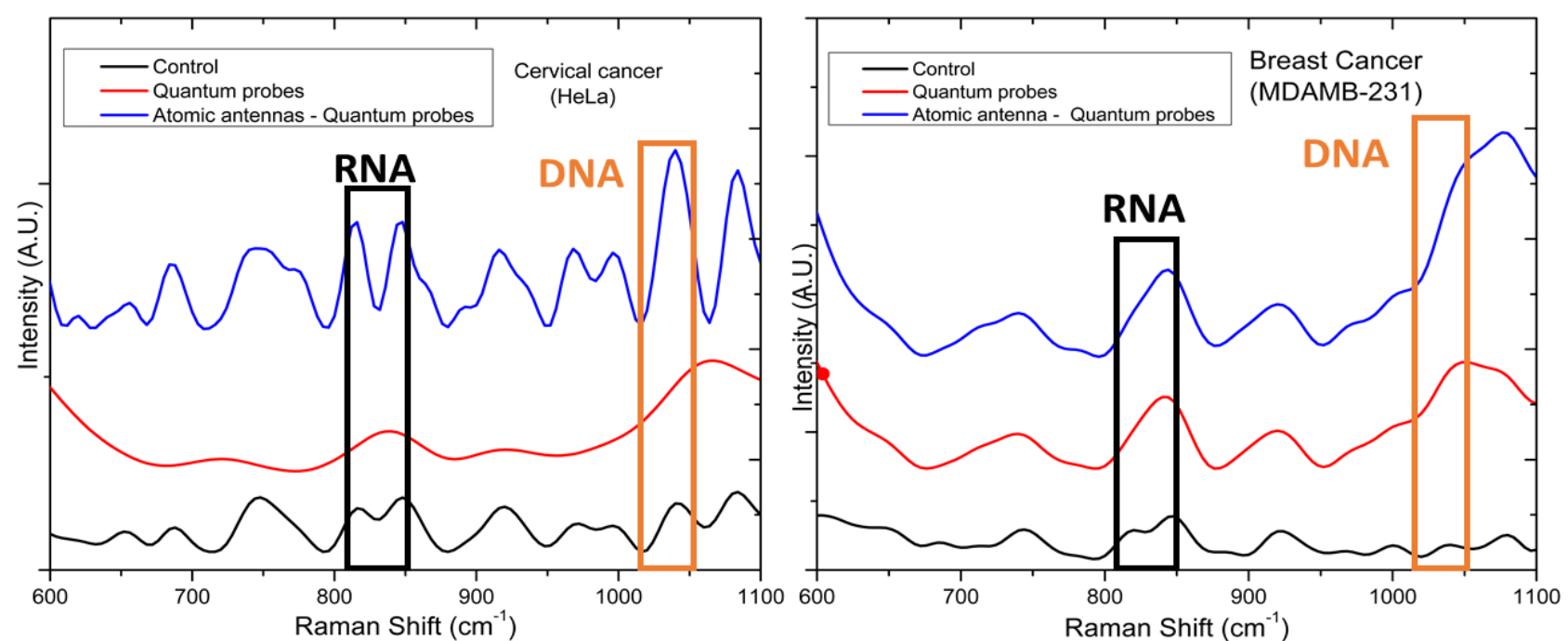

Atomic antenna -Quantum probes

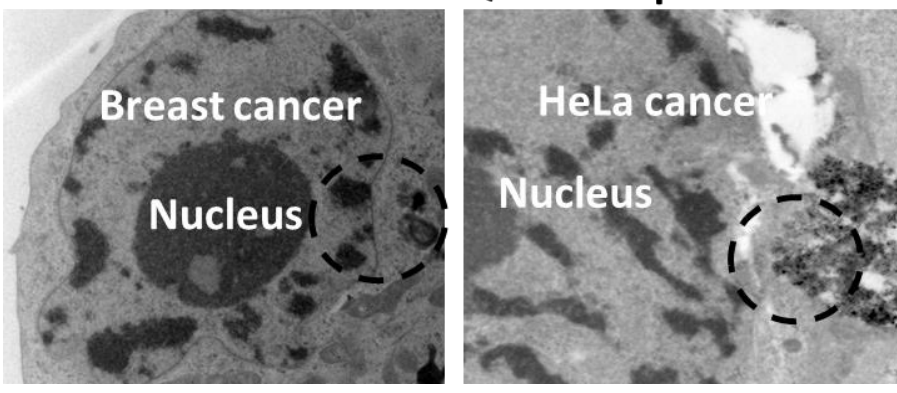

Quantum probes
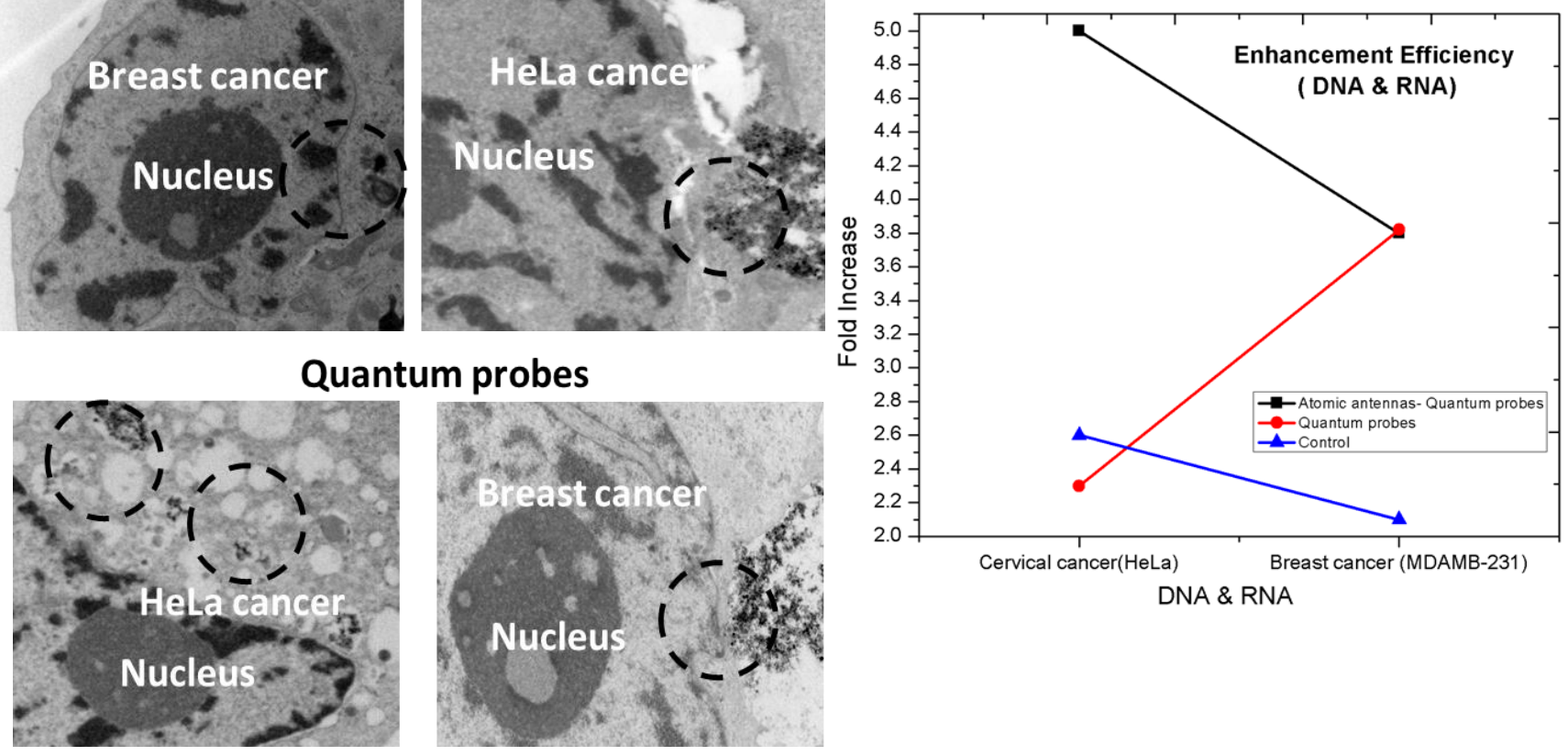

Figure 5-8: Detection of intracellular DNA and RNA of cells in the presence of quantum probes. The peak at $810 \mathrm{~cm}-1$ is identified as RNA outlined in black. The peak identified at $1066 \mathrm{~cm}-1$ is identified as DNA outlined in orange. The location of quantum probes is identified using black circular outline in the TEM images

uptake in cells and presence or complete absence of quantum probes inside the nuclear envelope from TEM observations. Both the quantum probes could precisely identify the change in both DNA and RNA intensity in HeLa and breast cancer cells. While comparing the sensitivity level between the probes with respect to DNA and RNA enhancement, the probes demonstrated 
distinct differences between them. For instance, the quantum probes with no atomic antenna could significantly sense the DNA and RNA changes in breast cancer cells when compared with HeLa cancer cells. Also, we observed a significant drop in RNA peak intensity at $810 \mathrm{~cm}^{-1}$ for HeLa cancer cells. The same difference was however not seen in breast cancer cells. This fact suggests and supports the use of RNA for protein synthesis during initial states of cell growth. The quantum probes with atomic antennas did showcase a reversal in DNA and RNA sensitivity when compared with quantum probes with no atomic antennas. This is validated by the 3-fold increase in DNA sensitivity of quantum probes with atomic antennas for HeLa cancer cells when compared with quantum probes with no atomic antennas. Furthermore, based on the reactivity of both the quantum probes, we could infer that quantum probes with atomic antennas has the potential to act as primary detector in sensing precise changes in HeLa cellular constituents.

Another remarkable aspect is the change in DNA peak intensity observed in the Raman spectrum. The decrease in DNA peak intensity is suggestive of cells undergoing apoptosis. It can be inferred from the drastic drop in DNA peak intensity of both HeLa and MDA-MB 231 cells, the quantum probes with atomic antenna could also stimulate cancer cells to undergo apoptosis. The drop in DNA Raman peak intensity could be attributed to disintegration or to chromatin condensation observed in the TEM images from figure 5-8.

\subsubsection{Cancer monitoring/therapeutics efficiency:}

To better understand and investigate the cell endocytosis behavior and corresponding Raman response, a time-based Raman response was conducted at 6, 12 and 24 hours. The TEM images from figure 5-9 does show the presence of quantum probes in the nucleus even after 24-hour 

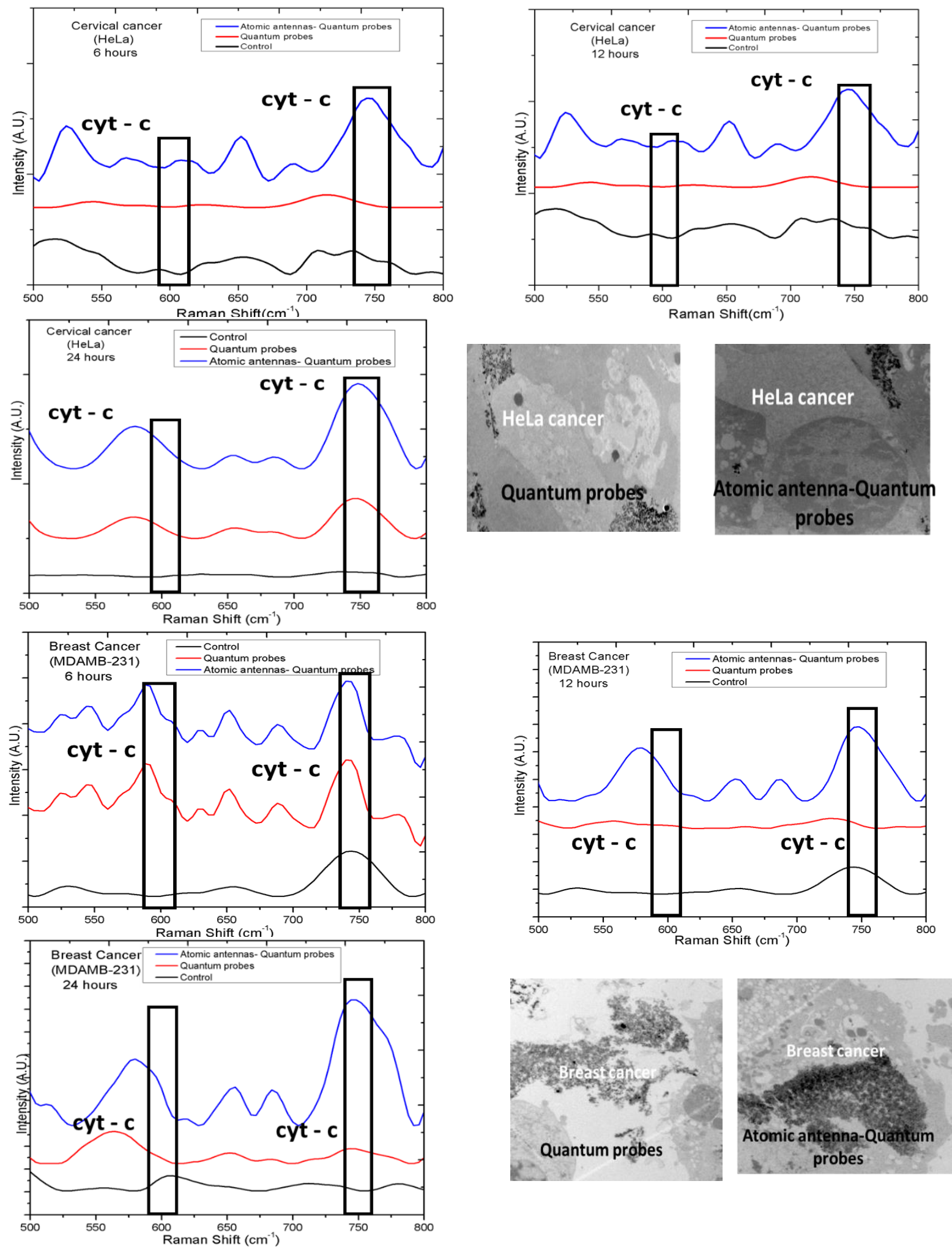

Figure 5-9: In-vitro Raman response to Quantum probes interaction with HeLa and breast cancer cells over 6, 12 and 24 hours. The cytochrome c peak positions are boxed in black outline. The corresponding TEM images show the quantum probes uptake in both cancer cells after $\mathbf{2 4}$ hours 
incubation. This corroborates most current assumptions that the probes must be in contact with the interacting cell organelles to induce Raman response. The increased accumulation and presence of quantum probes in the cancer cell cytoplasm from figure 5-9 also corresponds with the measured Raman spectra in cell. For cellular analysis the Raman spectrum must exhibit sharp narrow peaks in the Raman fingerprint region of 600 to $1800 \mathrm{~cm}^{-1}$ from Lu Wei et al., ${ }^{213}$. The internalized quantum probes have now provided a broad range of biochemical information pertaining to the analysis aspect including the presence of certain constituent biochemical elements.

The Raman peaks at 605 and $750 \mathrm{~cm}^{-1}$ are together assigned to vibrational of pyrrole breathing mode in cytochrome c. Cytochrome $\mathrm{c}$ is a protein complex which is reported to play a significant role in determining cell apoptosis. Since, it absorbs light at $\sim 530 \mathrm{~nm}$ which in turn shows strong resonance scattering, $532 \mathrm{~nm}$ Raman laser excitation cannot be used to determine cytochrome c activity in cells. This justifies the need for $785 \mathrm{~nm}$ Raman laser for cancer cell monitoring and therapeutic response. Furthermore, cytochrome is a constituent element in cell mitochondria. Thus, the Raman peak observed in cervical cancerous cell (HeLa) at 605 and $750 \mathrm{~cm}^{-1}$ are from cytochrome $c$ present in the cell from available literature ${ }^{214}$.The presence of these characteristic peaks can be used as a label-free marker to determine cancer cell apoptosis. The cytochrome c peaks are initially observed at 6 hours on both quantum probes, but was completely absent in the native control suggesting the quantum probes used in this study can identify the cancerous cell apoptosis. An increased incubation time from 12 to 24 hours, further evidenced increased presence of cytochrome c peaks. Furthermore, both quantum probes were also able to pick up Raman peaks associated with cytochrome $\mathrm{c}$ present in both HeLa and breast cancerous cells. This 
reaffirmed the ability and versatile capability of the portable quantum probes to act as real-time cancer cell monitoring agents in an in-vitro environment.

\subsection{CONCLUSION:}

Herein, we successfully introduced a label-free non-TERS approach to an in-vitro cancer detection and diagnosis process by using nickel based quantum probes with atomic antennas. By reducing the size of the probes from nano to quantum scale, we attained an ultra-sensitive quantum probes capable of sensing of femtomolar concentrations of Raman active dyes such as CV and R6G with $10^{12}$ enhancement efficiency. The in-vitro sensing ability was demonstrated by BSA biomolecule sensing at nanomolar concentrations. The ability of quantum probes to in-vitro cancer cell detection was established using a time-based series analysis from 6 to 24 hours. The quantum probes with atomic antennas also exhibited a passive endocytosis uptake mechanism where the cancer cells being aggressive nature displayed increased quantum probes uptake which helped in primarily to detect and distinguish cancer cells from those of the fibroblast cells. This was achieved with variation in intercellular protein enhancement. The atomic antennas in quantum probes also displayed increased DNA and RNA sensitivity between the investigated cancer cells, thus showcasing its cancer cell differentiation ability. In-addition, to demonstrate the continuous cancer cell monitoring and prognosis the quantum probes helped in label-free detection of increased concentration of apoptotic protein marker cytochrome c from 6 to 24 hours. The quantum probes with and without atomic antennas were engineered using programmable multiphoton ionization mechanism. The label-free cancer detection, 
differentiation and prognosis with significant Raman enhancement established in this study, thus hold potential for an effective non-invasive cancer detection and treatment in future. 


\section{CHAPTER 6}

\subsection{CONCLUSion ANd FutURe ReSEARCH:}

In this thesis, creation of a self-assembled 3D hybrid nickel nanonetwork primarily for biomolecular sensing subsequently for both cancer therapeutic and theranostics application is presented. The unique structure engineered and generated had Raman enhancing and therapeutic characteristics with tunable physiochemical property which was achieved using ultrashort pulsed laser through deployment of multiphoton ionization process. Nickel is a prime magnetic transition material well known for its excellent biocompatibility unfortunately till date finds extensive application only in the field of MRI diagnosis and in cancer theranostics as a primary drug carrier.

Upon our selection to have nickel as a metal of our choice in our research study, we noted that there exists a vacuum in utilizing nickel based nanomaterials for cancer treatment. Our first step in the research involved the synthesis of nickel nanonetwork platform which combines and inherits all the unique properties of self-assembled 3D nanonetwork with its complex morphology to effectively regulate HeLa cancer cell adhesion and proliferation. The synthesized 3D nickel nanonetwork demonstrated Extra Cellular Matrix (ECM) like behaviour during the initial incubation period of 24 hours by continuously baiting fibroblast and HeLa cells to proliferate upon the engineered 3D nanonetwork. However, the same cuboidal nickel 3D nanonetwork after an increased incubation exposure period of 48 hours exhibited an unique cell trapping behaviour only upon HeLa cells thereby effectively controlling proliferation and stunting further growth of cancer cells. This unique aspect noted, result derived and recorded added a new dimension as to how HeLa cells behaved quite differently when compared to NIH3T3 fibroblast cells on nickel 3D nanonetworks. It can therefore be clearly inferred that this specific aspect of controlled proliferation and stunting the growth of cells is feasible using the 3D self-assembled nickel nanonetwork. Therefore, our engineered and specifically structured 3D nanonetwork has the desired potential 
application that we are looking for application in the field of health care and is a right choice to develop a drug-free cancer theranostics nano medicine.

In the second phase of our ongoing research study, the focus was on identifying and declaring that the same Nickel based theranosomes are compatible for usage in cancer theranostics application. Hence our study was directed towards evolving and deriving a potential quantum medicine as a result by activating the therapeutic functionality of both $\mathrm{Ni}$ and $\mathrm{NiO}$ at quantum scale level by altering the basic material chemistry through quantum scale engineering process. The study therefore focussed on those linesso as to generate a 3D scalable magnetic quantum theranosomes by deploying multiphoton ionization process upon a nickel substrate. Apart from the formation of these 3D quantum theranosomes, additionally a label-free bioimaging probes, which has so far been conventionally used in the field of bioimaging was also generated. An intrinsically non-bioluminescent bulk nickel substrate was then transformed to create this 3D quantum theranosomes, which its self was unique on its own as it had all the features and properties to overcome the current major drawbacks such as cytotoxicity, targeted delivery and particle size dependency. Incidentally all the aforesaid drawbacks were faced during the application of the conventional and freely available QD. Upon confirmation that the above cited drawbacks when using primary (quantum - $\mathrm{Ni}$ ) and secondary (quantum - $\mathrm{NiO}$ ) theranosomes, are fully addressed, we launched our focus on further evaluating the cellular interactions between the synthesized magnetic theranosomes and cancer cells. We were more specific in analysing the behaviour and effect these quantum scale materials have on the two most important aspects namely cellular proliferation and cytotoxicity as these two form the fundamental aspect in any type of cancer therapy. We demonstrated that the theranosomes only selectively accelerated the proliferation of mammalian fibroblasts cells, but not that of the cancer cells essentially acting as Extracellular Matrix (ECM) thereby inducing a very negligible cytotoxicity. In addition, the in-vitro study revealed that the cancerous HeLa, MDAMB-231 and NIH3T3 fibroblast cells had a higher propensity of particle uptake thereby allowing diagnostic imaging too though not only a 
critical factor but was a much-desired feature. Additionally, when we synthesized theranosomes probe we observed that a very significant auto fluorescence intensity under Flow cytometry was also present when compared with other scalable nickel structures. This specific citing noted in our research study brought out an entirely new dimension to confirm that a theranosomes probe we had engineered and generated can be effectively be used as a single system application for both bioimaging (diagnostics) and for cancer therapy. To our best of knowledge this kind of a single system application has not yet been reported so far opens up new vistas that 3D nickel based magnetic quantum theranosomes can now be specifically engineered and tailor made to meet the targeted application. We anticipate that our findings noted and recorded by these unique 3D nickel based magnetic quantum theranosomes can further be extended for other generic application in the field of quantum medicine.

In the third phase of our research study, we noted the need for an ultrasensitive biosensor for targeted molecular sensitivity that overcomes the potential drawback of fluorescence sensing. As a first step in our venture was to carry out a detailed study on the aspects of the proposed ultrasensitive biosensor. We developed a Raman active nano-biosensor using nickel nanonetwork created by multiphoton ionization process as a platform/scaffold to detect chemical dye Crystal Violet (CV) and biomolecule glutathione(GSH). The Raman detection of crystal violet (CV) and glutathione (GSH) molecules at 1 pM ( 1 $\times 10^{-12} \mathrm{M}$ ) concentrations were made at two excitation wavelengths $532 \mathrm{~nm}$ and $785 \mathrm{~nm}$. An enhancement factor of $10^{9}$, a level that had so far not been observed till date even with noble metal monolayer coatings was sighted while conducting our experimentation process. This aspect extends the present threshold regarding the limit of detection (LOD), making these self-assembled 3D hybrid nickel nanonetwork generated as a very promising "non-noble metal" based candidate for future chemical and biomolecular sensing applications. With the results noted, recorded and now presented in this thesis, a very new role has now been earmarked and defined for these Ni nanostructures as a biosensor and that too upon a therapeutic platform. It should be noted that not only has the SERS enhancing properties of $\mathrm{Ni}$ been 
activated by the unique 3D nanonetwork structure but the cancer therapeutic aspect of the synthesized Ni nanostructures observed far exceeds the multifunctionality of any other conventional nanomaterials currently available. Hence our current research study findings now reported paves way to open up further additional new avenues in the field of cancer theranostics application.

\subsection{MAJOR CONTRIBUTION:}

In this concluding summary, we present a salient highlight of the new findings wherein a self assembled 3D nickel nanoparticle matrix generated using ultrashort pulsed laser served not only the primary function by distinguishing and differentiating cancer cells from those of the fibroblast cells but thereafter emerged as a platform exhibiting fibroblast cell proliferation and induce apoptosis to HeLa cancer cells by a single step process application. Furthermore, a theranostics bioimaging probe that was developed was synthesized using primary and secondary theranosomes so as to specifically diagnose and distinguish cancer cells from those of the fibroblast cells while simultaneously inducing selective cancer cell apoptosis. In-addition, the SERS active platform engineered and structured culminated in the development of a new generation of nickel nano-biosensor which can now be used for biomolecular sensing applications. By this new generation of biosensor both the cancer diagnosis and its further specific treatment are made totally simple as the cell viability assessment study conducted as a part our research study had further proved the effectiveness in specific and accurate cell differentiation.

\subsection{FutURE RESEARCH:}

With the development of these Ni-based nanostructures that have substantially high therapeutic efficiency and SERS enhancement, the possibility of further developing Ni based nanomaterials is quite immense and further enrichment of this approach with additional biosensing nanomaterials is indeed an extremely interesting new research avenue to be ventured. The following future objectives represent 
potential advancements that can still be made using the multiphoton laser ionization mechanism for $\mathrm{Ni}$ based nanostructures.

\subsubsection{In-vitro cancer detection of cellular components using label-free nickel nanomaterial:}

Since initial discovery of surface-enhanced Raman spectroscopy (SERS) in 1970s, the use of SERS based chemical and biological compounds has grown at an exponential pace. Recent progress in analytical chemistry has resulted in generating functional DNA sensitive material by using SERS phenomenon. Currently, all reported SERS active DNA sensing substrates employ noble metals like gold or silver to detect and enhance DNA molecules. The use of noble metals hinders enhancement process as sensing is limited to non-metal active analytes. Thus, SERS-active nanostructures have been synthesized in the past from our group on Raman inactive materials like titanium and silicon ${ }^{24,61}$. However, the use of these SERS active synthesized nanomaterial for protein and DNA sensing has never been reported before. We plan on employing the fabricated SERS active nickel nanonetworks to target protein and DNA molecule in cancer cells without employing additive materials like nanogold/colloidal golds to induce SERS response. This helps in achieving a non-metal based SERS active probes which can be extended to several cancers and biosensors thereby making diagnostics more efficient and viable to all. Through this thesis, $\mathrm{Ni}$ nanostructures have been proven to be ultrasensitive SERS platforms for the detection of biomolecules. In this proposed future work, the use of these Ni nanostructures will be expanded to establish these nanostructures as SERS sensitive substrates for label-free cancer diagnosis.

\subsubsection{Examine nickel nanonetwork influence in Co-Cultured In-Vitro environment:}

Studying the interaction of fabricated nickel nanonetwork comprising of a wide range of multiple cell types allows us to understand fundamental subcellular biological processes and reactions such as simultaneous intercellular signalling between multiple cells with synthesized nanonetwork. All earlier studies were 
conducted only upon harmonized cell types available in 3D nanostructures. Extension of this study would definitely enable us to simulate the in-vivo environment thereby taking the cancer theranostics further, so as to mimic or replicate the natural human body conditions.

APPENDIX - A

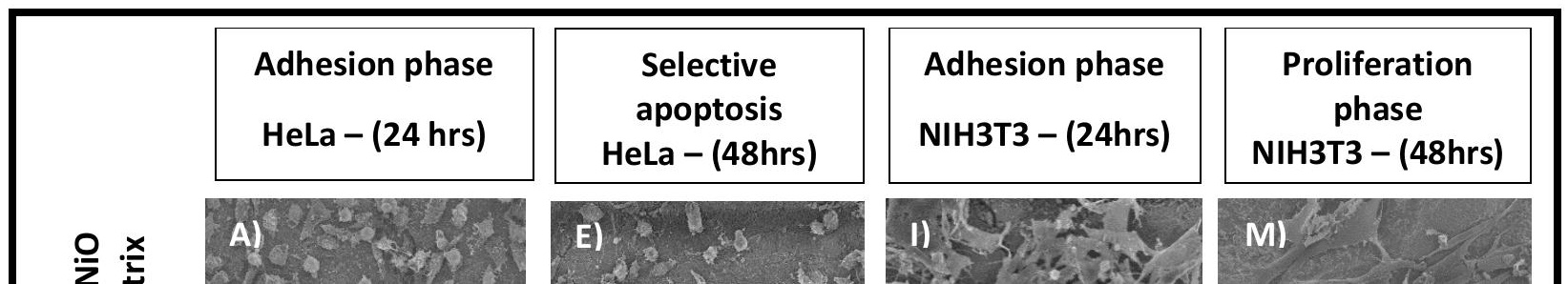



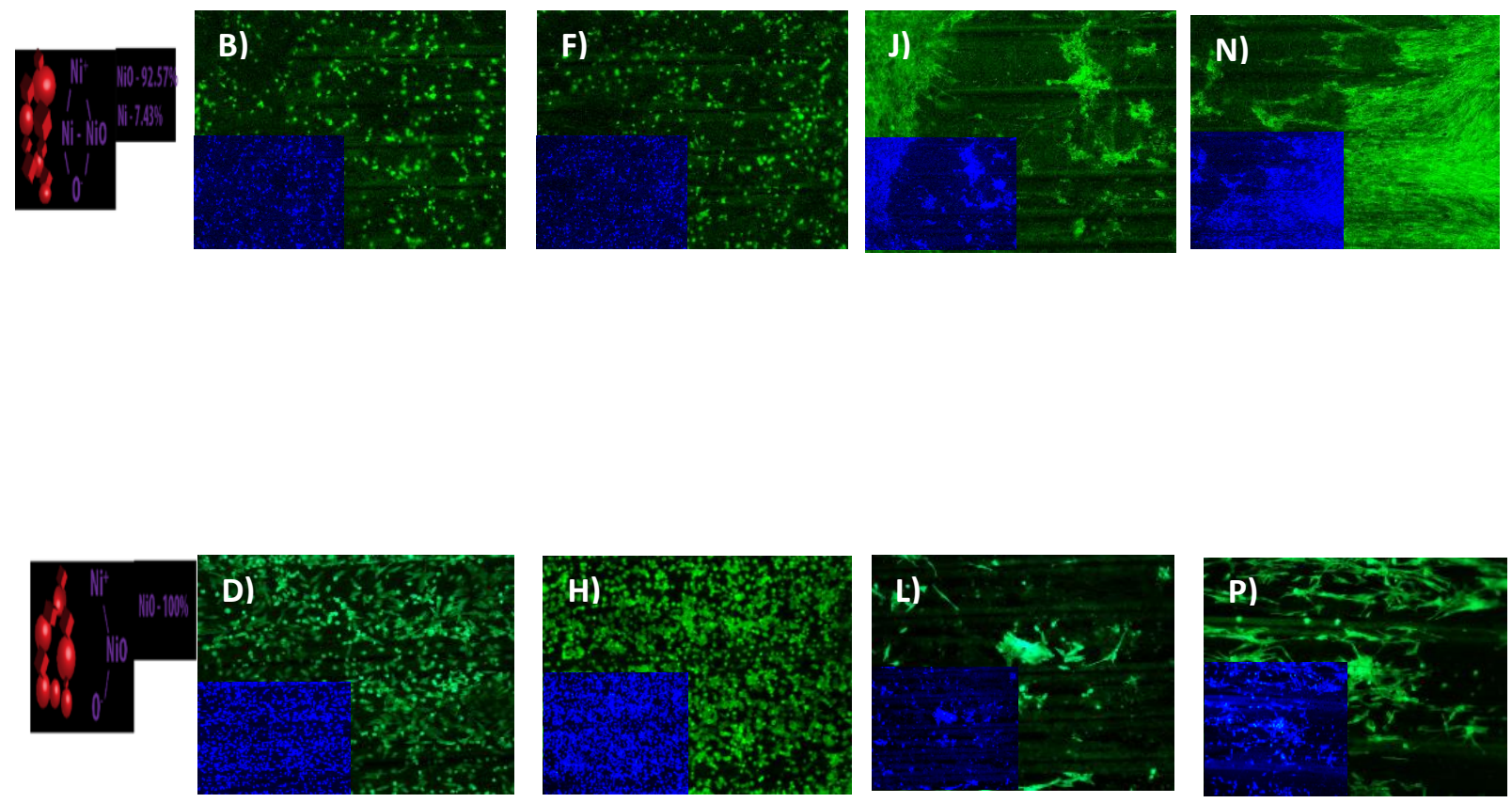

Figure A-S1: Quantifying HeLa and NIH3T3 fibroblast cell proliferation on synthesized nanomatrix for 24 hours and 48 hours. (A-D, E-H, I-L, M-P) fabricated platforms. Images $(B, D, F, H, J, L, N, P)$ have a uniform scale of $10 \mu \mathrm{m}$ while the rest has a uniform scale of $100 \mu \mathrm{m}$. 


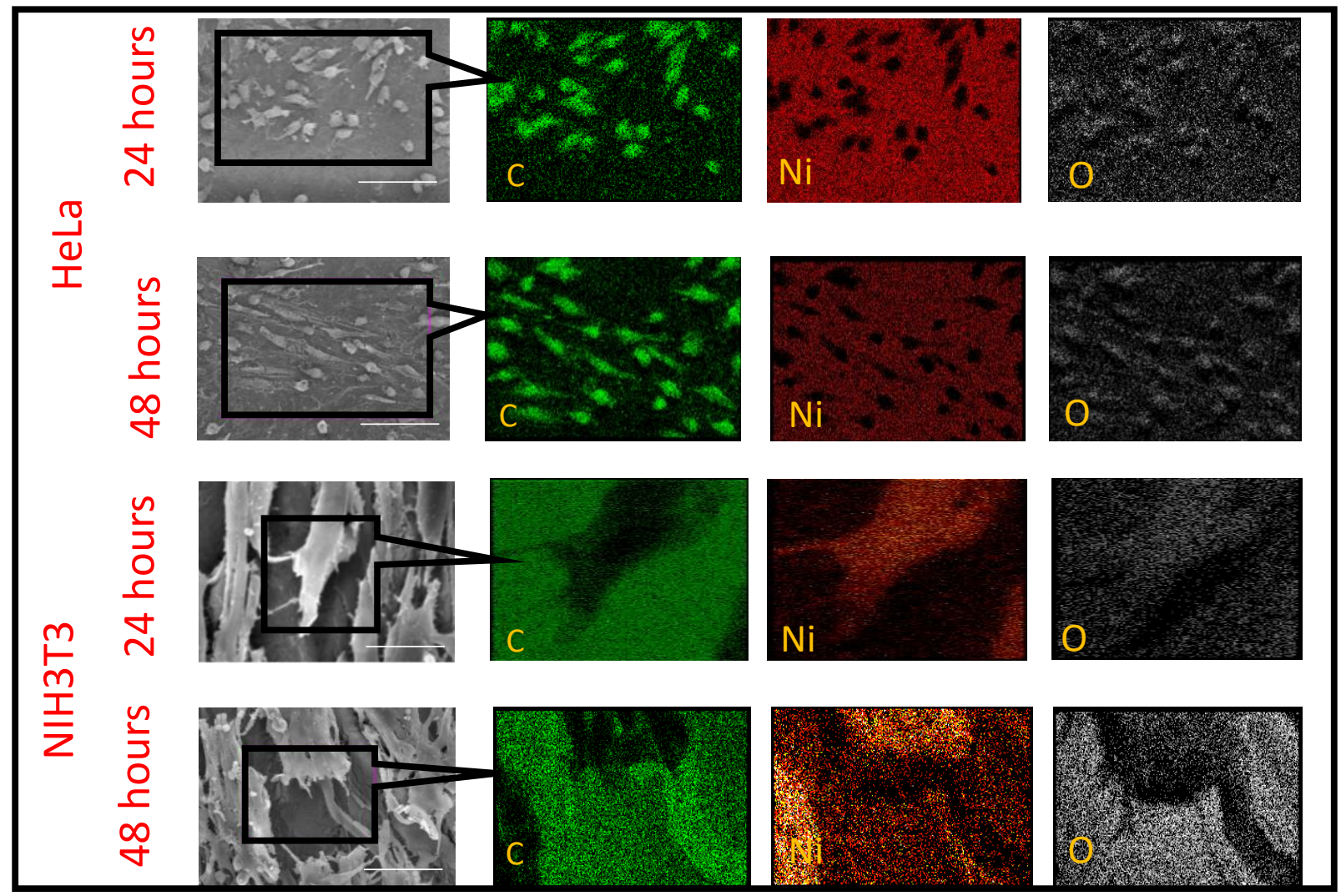

Figure A-S2: Nanoparticle in $100 \%$ NiO nanomatrix interaction with HeLa and NIH3T3 fibroblast cells. SEM image and EDX elemental mapping of oxygen (green), Nickel (Red) and Oxygen (grey). All the images have a uniform scale of $100 \mu \mathrm{m}$ 


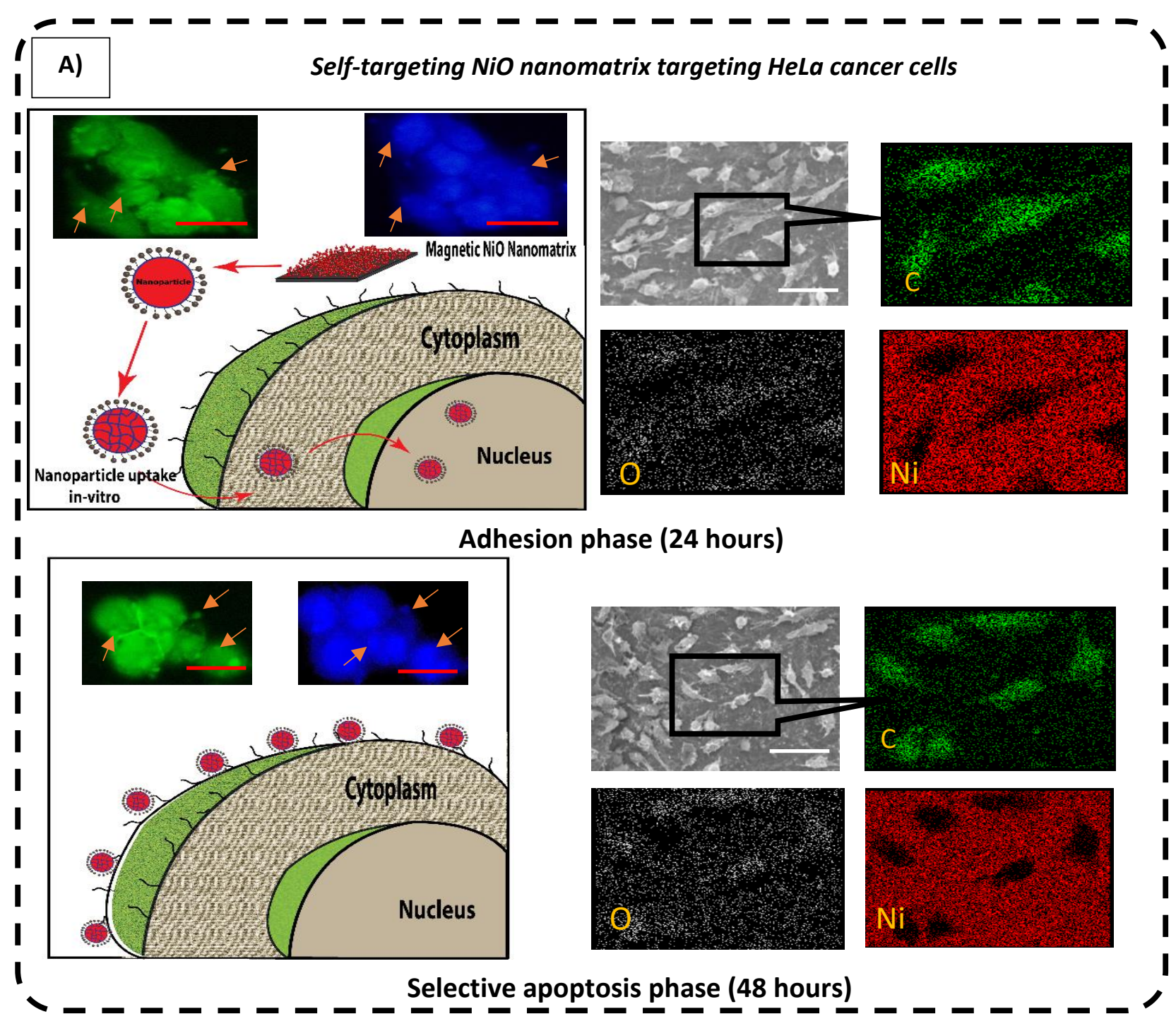




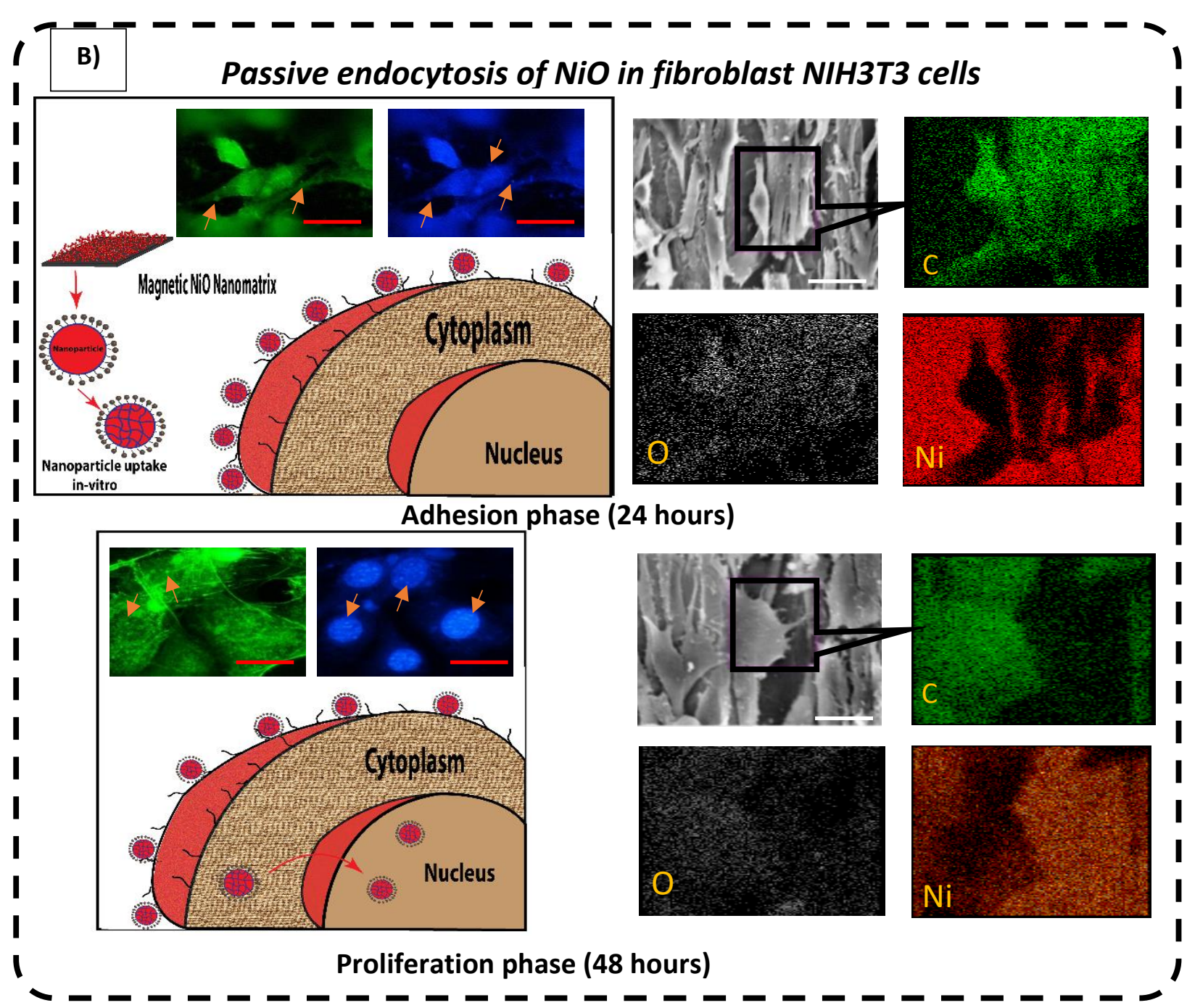

Figure A-S3: Elemental Energy Dispersive X-ray (EDX) mapping on nanoparticle interaction with cells at $67.29 \%$ NiO nanomatrix A) HeLa and B) NIH3T3 cells. A) Nanoparticle in $67.29 \%$ NiO nanomatrix interaction with HeLa and NIH3T3 fibroblast cells. SEM image and EDX elemental mapping of oxygen (green), Nickel (Red) and Oxygen (grey). All the images have a uniform scale of $100 \mu \mathrm{m}$. B) Nanoparticle in $100 \%$ NiO nanomatrix interaction with HeLa and NIH3T3 fibroblast cells. SEM image and EDX elemental mapping of oxygen (green), Nickel (Red) and Oxygen (grey). All the images have a uniform scale of $100 \mu \mathrm{m}$ 
APPENDIX - B
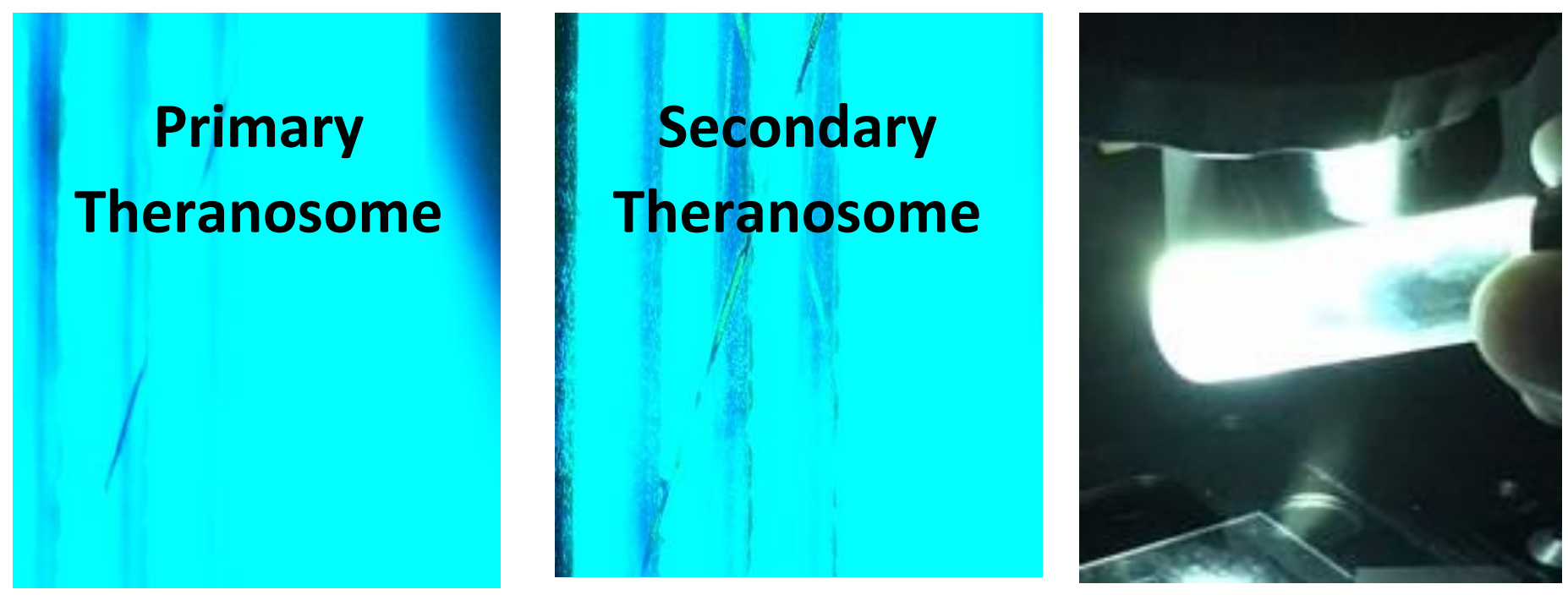

Figure B-S1: White Light Emission at Primary and Secondary Theranosomes along with a representative broadband emission

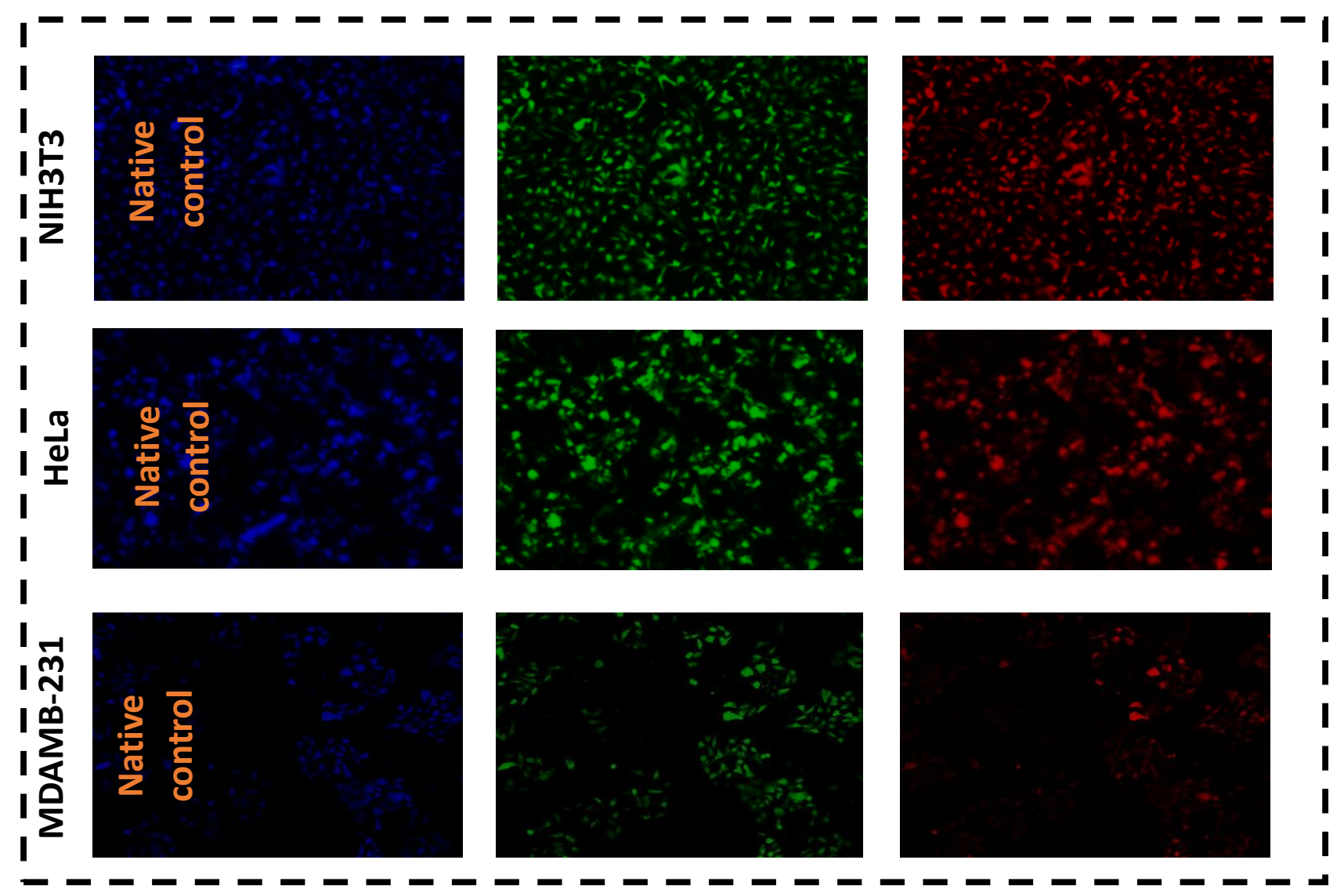

Figure B-S2: Fluorescence Intensity of NIH3T3, HeLa and MDAMB- 231 cells upon native controls. All the images are set to a standard scale of $10 \mu \mathrm{m}$. 

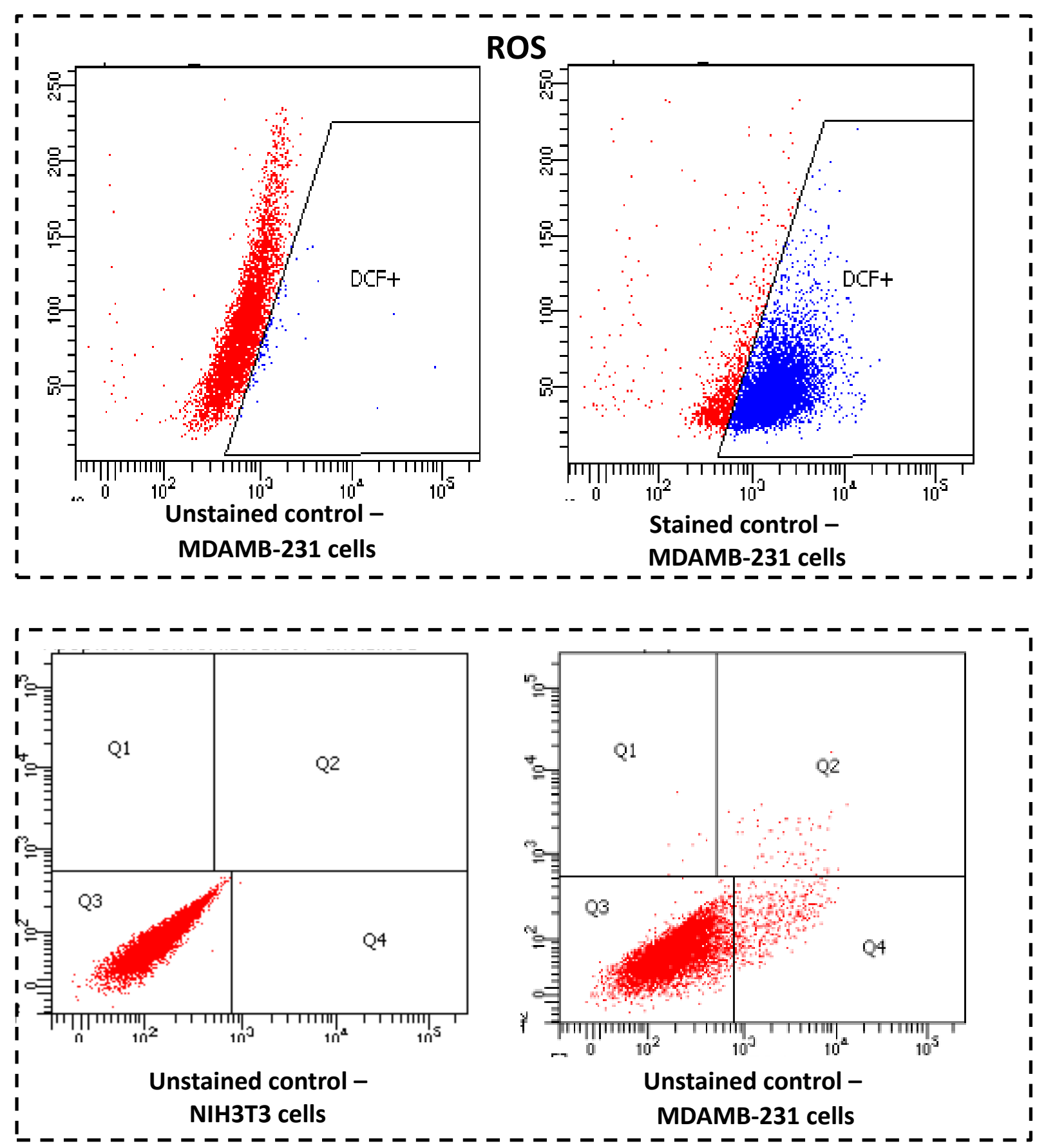

Figure B-S3: Stained and unstained control for ROS and Apoptosis assessment 


\section{APPENDIX - C}

\section{Raman Enhancement factor calculation}

The Raman enhancement factor (EF) for 3-D nanostructures is analyzed experimentally using the following Raman EF equation present in most accepted literature ${ }^{142,171,202}$.

$$
E F=\frac{I_{\text {nanonetwork }} / N_{\text {Surface }}}{I_{\text {substrate }} / N_{\text {bulk }}} \quad \text { Eq.S1 }
$$

$I_{\text {nanonetwork }}$ represents the Raman SERS intensity on nickel nanonetwork, $I_{\text {substrate }}$ represents the Raman intensity on nickel substrate, $\mathrm{N}_{\text {bulk }}$ represents the number of molecules present under the confocal volume and $\mathrm{N}_{\text {surface }}$ represents the number of molecules responsible for the SERS response. A $10 \mu$ l volume of dye is added on nickel nanonetwork and nickel substrate for evaluating SERS response. From Figure S3, $I_{\text {nanonetwor }}=4955$ and $I_{\text {substrate }}=131$ at $1620 \mathrm{~cm}^{-1}$. The CV molecule has strong vibrational modes at $1620 \mathrm{~cm}^{-}$ ${ }^{1}$ wavenumber and is used in EF calculations ${ }^{203}$. The following equation is used to calculate $\mathrm{N}_{\text {bulk. }}$.

$$
N_{\text {bulk }}=\pi r^{2} h c N_{A} \quad \text { Eq.S2 }
$$

Where $\mathrm{N}_{\mathrm{A}}$ is Avogardo constant, $\mathrm{r}$ represents the Raman laser spot radius $(1.5 \mu \mathrm{m}), \mathrm{h}$ is the half the depth of Field (DOF) of a 50x magnified laser used in the study $(2 \mu \mathrm{m})$, c represents crystal violet (CV) appropriate concentration $\left(1 \times 10^{-3}, 1 \times 10^{-6}, 1 \times 10^{-9}\right.$ and $\left.1 \times 10^{-12}\right) \mathrm{M}$ was dropped on the substrate. $\mathrm{N}_{\text {bulk }}$ is calculated using equation S2 and to calculate $\mathrm{N}_{\text {surface, }}$ surface area and CV absorption on nickel nanonetworks are necessary. Some conservative estimates of nanonetwork were made in calculating $\mathrm{N}_{\text {surface }}$ from the work of Maznichenko et al., and Powell J.A. et al., as a very similar ion plume formation mechanism is employed to create $\mathrm{TiO}_{2}$, silicon and nickel nanonetworks ${ }^{24,61}$. The density of the nanonetworks is assumed to be identical to bulk nickel $\rho=8.90 \mathrm{~g} / \mathrm{cm}^{3}$ and BET surface area as $24 \mathrm{~m}^{2} / \mathrm{g}$ from literature ${ }^{204}$. The SERS experiments were carried out immediately after CV dye application on nickel nanonetwork and nickel substrates. To approximate the number of molecules that contribute to Raman enhancement from the 
nickel nanonetwork, we need to consider the number of dye molecules adsorb onto surface of nanonetwork. Since, it is not practical to weigh cubic micrometer volumes a similarity relationship is established. For both $\mathrm{N}_{\text {bulk }}$ and $\mathrm{N}_{\text {surface }}$ we have assumed only the molecules present within the interaction volume of the Raman laser contribute to Raman enhancement. $\mathrm{N}_{\text {surface }}$ is calculated using the equation S3,

$$
N_{\text {surface }}=S A_{\text {eff }}\left(C_{a d s} N_{A} \frac{1000 L}{1 m^{3}}\right)^{2 / 3} \quad \text { Eq.S3 }
$$

Where $S_{\text {eff }}$ is the effective surface area of nanonetwork within Raman laser interaction volume, $C_{\text {ads }}$ is the surface adsorption of the dye molecule and $\mathrm{N}_{\mathrm{A}}$ is Avagardo's constant. Finally, the enhancement factor is calculated using equation s1.

Table C S1 - Comparison of laser parameters with synthesized nanonetwork nomenclature

\begin{tabular}{|c|c|c|}
\hline Laser parameters & Nomenclature & Average nanoparticle size \\
\hline $25 \mathrm{MHz} 214 \mathrm{fs} 5 \mathrm{~ms}$ & Cuboidal nanonetwork & $10.14 \mathrm{~nm}$ \\
\hline $12 \mathrm{MHz} 214 \mathrm{fs} 5 \mathrm{~ms}$ & Spheroidal-Cuboidal nanonetwork & $15.241 \mathrm{~nm}$ \\
\hline $4 \mathrm{MHz} 214 \mathrm{fs} 5 \mathrm{~ms}$ & Spheroidal nanonetwork & $19.059 \mathrm{~nm}$ \\
\hline
\end{tabular}




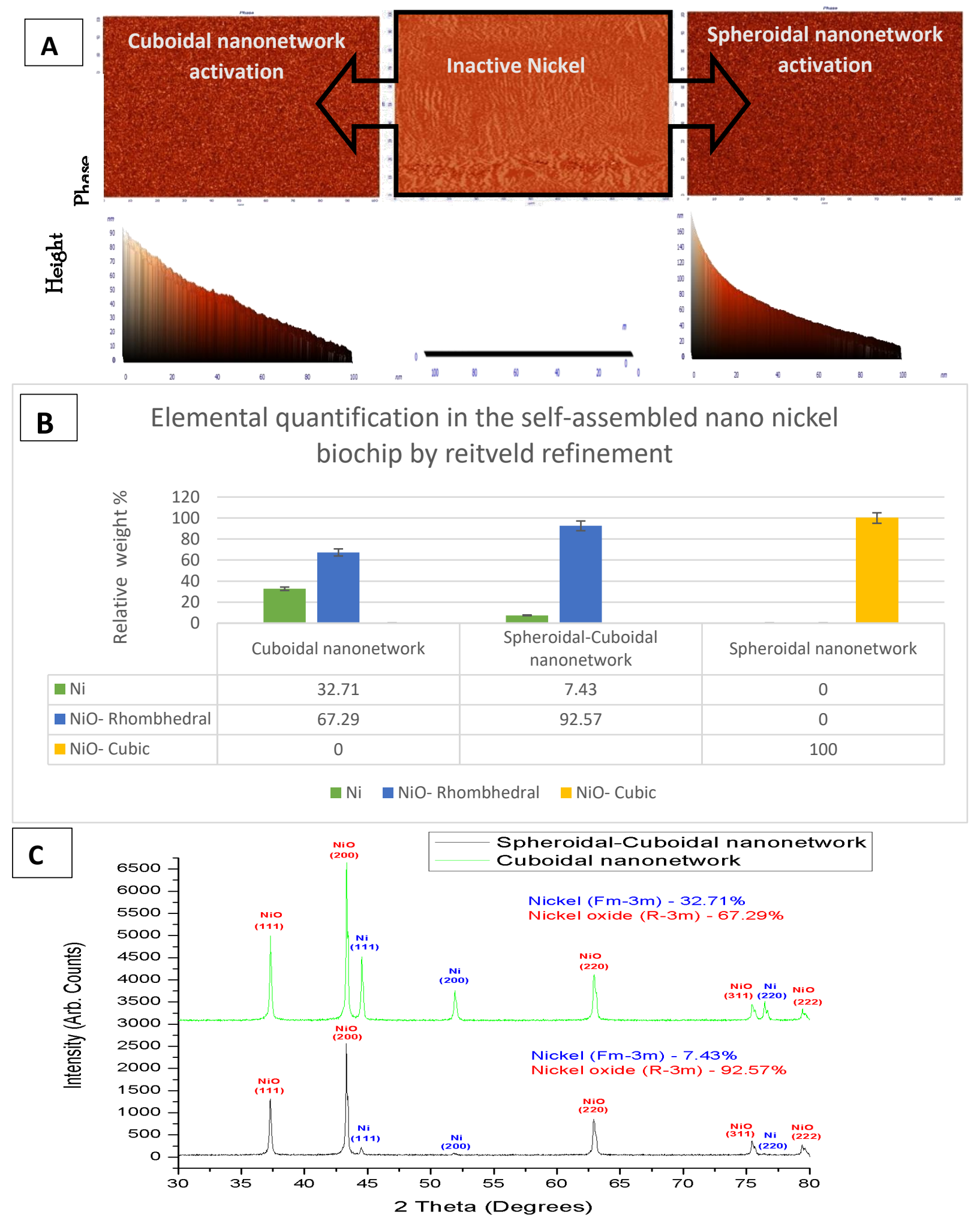

Figure C-S1(A) AFM image of height and phase analysis on synthesized nanonetwork along with inactive native nickel substrate. A uniform scale bar is 100 mm is followed. B) Reitveld refinement of synthesized nanonetwork. C) XRD pattern of at cuboidal and spheroidal nanonetwork. 


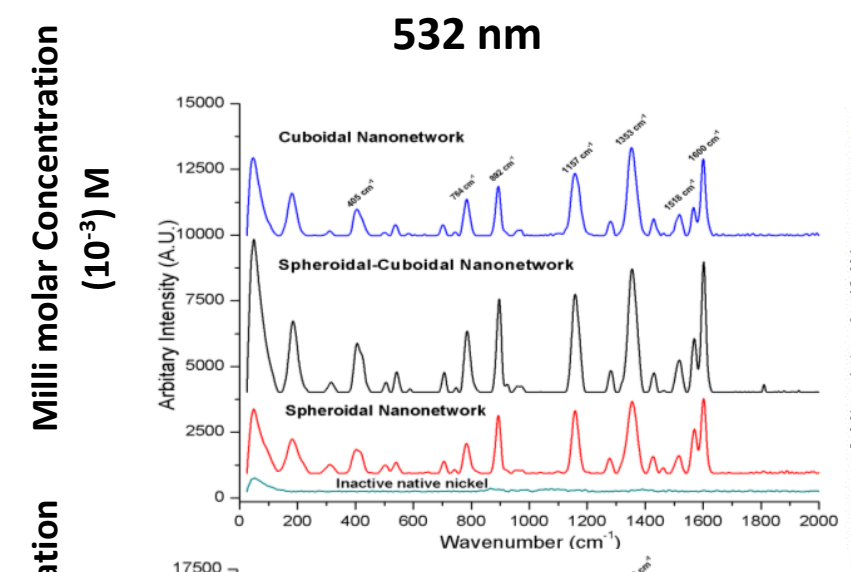

$532 \mathrm{~nm}$

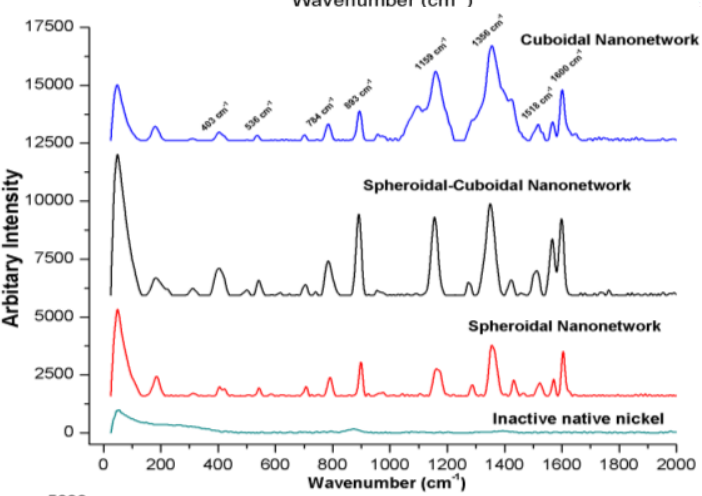

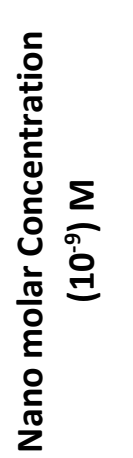

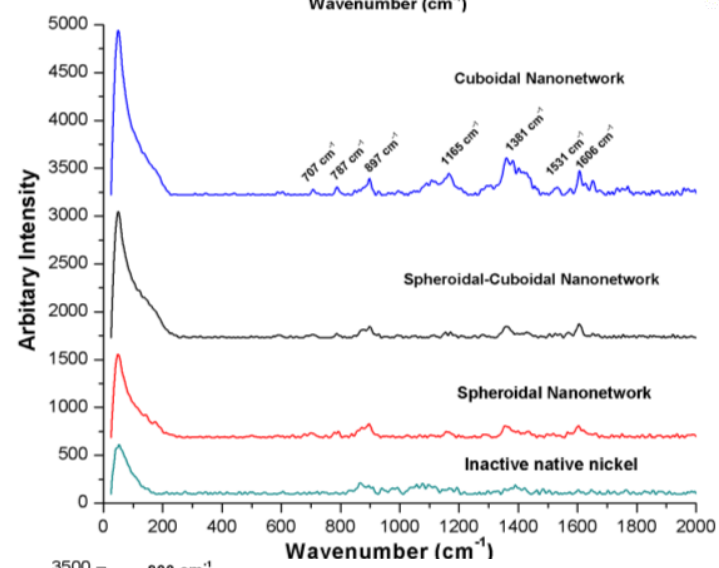

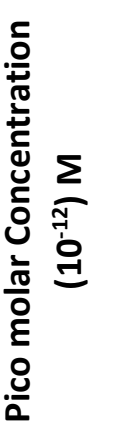

\section{$785 \mathrm{~nm}$}
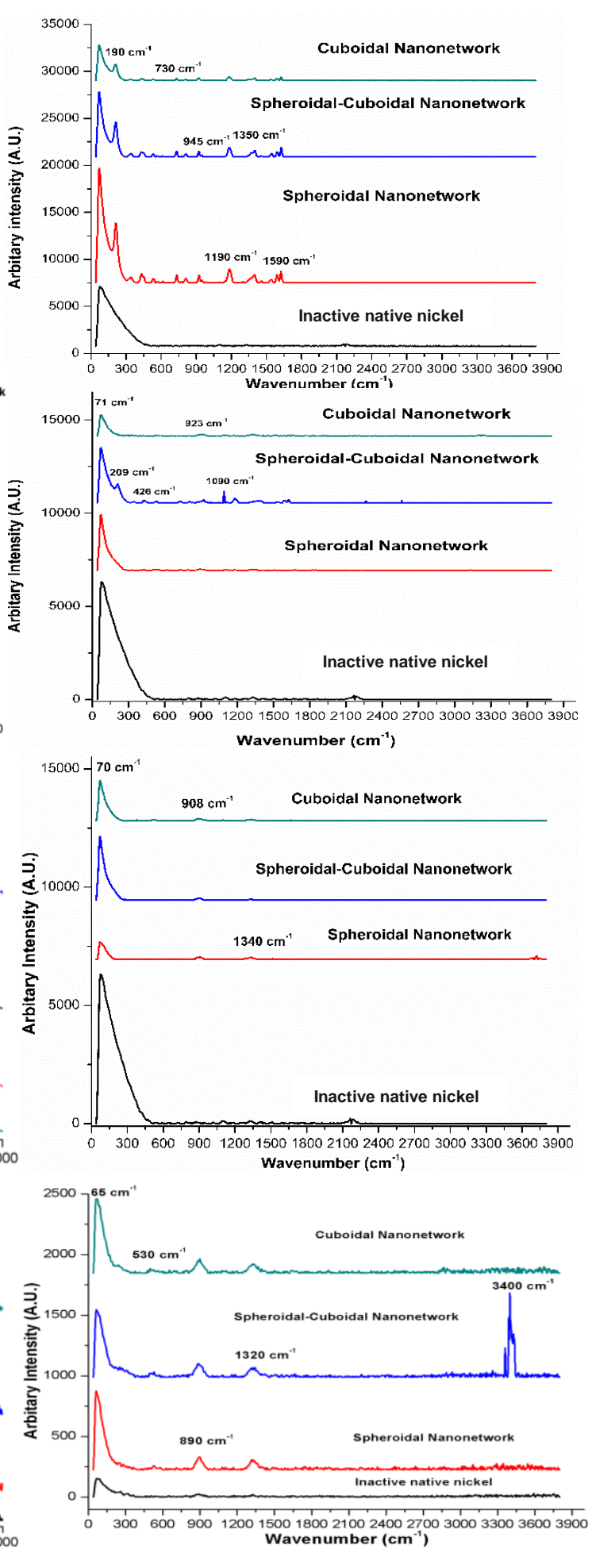

Figure C-S2: Activated nickel nanonetwork in nickel biochip interaction with Crystal Violet (CV) at dual (532 nm) \& (785 nm) Raman wavelength 

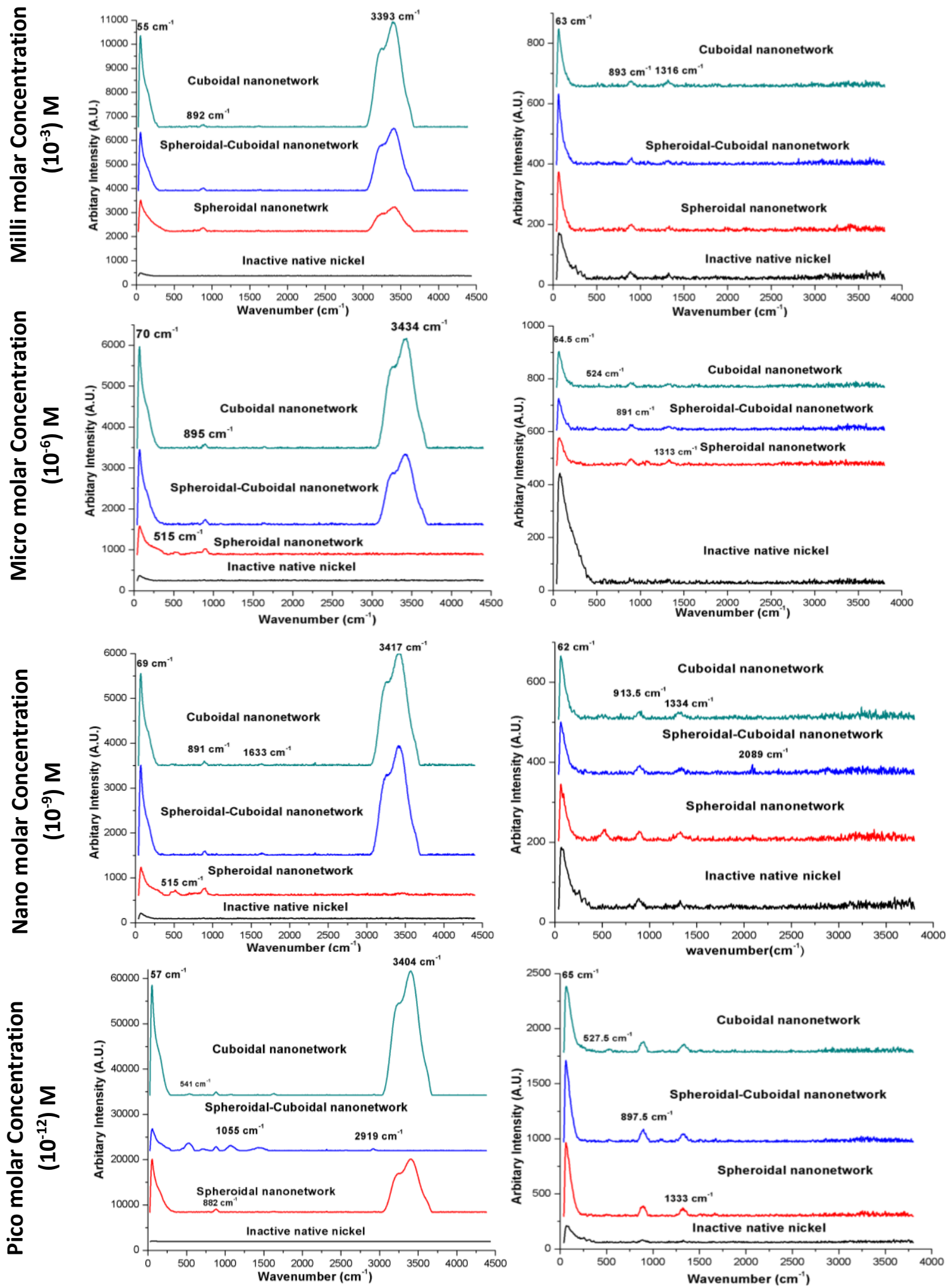

Figure C-S3: Activated nickel nanonetwork in nickel biochip interaction with biomolecule Glutathione (GSH) at dual (532 nm) \& (785 nm) Raman wavelength 
Table C S2: Raman excitation comparison based on nanonetwork composition vs GSH dye concentration

\begin{tabular}{|c|c|c|c|}
\hline $\begin{array}{c}\text { Nanonetwork } \\
\text { composition/Dye } \\
\text { concentration }\end{array}$ & Cuboidal nanonetwork & $\begin{array}{c}\text { Spheroidal-Cuboidal } \\
\text { nanonetwork }\end{array}$ & $\begin{array}{c}\text { Spheroidal } \\
\text { nanonetwork }\end{array}$ \\
\hline $1 \mathrm{mM}$ & Strong & Moderate & Weak \\
\hline $1 \mu \mathrm{M}$ & Strong & Moderate & Weak \\
\hline $1 \mathrm{nM}$ & Strong & Moderate & Weak \\
\hline $1 \mathrm{pM}$ & Strong & Weak & Moderate \\
\hline
\end{tabular}

Table C S3: A comparison of the proposed method with other methods for biomolecule detection

\begin{tabular}{|c|c|c|c|}
\hline Method & Material synthesized & Biomolecule used & Reference \\
\hline Electrochemical & $\begin{array}{c}\text { Capped gold } \\
\text { nanoparticles }\end{array}$ & Glutathione & 117 \\
\hline Fluorescence & $\begin{array}{c}\text { Carbon dots- } \mathrm{MnO}_{2} \\
\text { nanocomposites }\end{array}$ & Glutathione & 154 \\
\hline $\begin{array}{c}\text { Protein gated resonance } \\
\text { energy transfer }\end{array}$ & Gold nanoparticles & Cytochrome c & 19 \\
\hline Ligand exchange & Gold nanoparticles & Glutathione & 165 \\
\hline SERS & Silver nanoparticles & Glutathione & \\
\hline Our Method -SERS & $\begin{array}{c}\text { 3D nickel } \\
\text { nanonetwork }\end{array}$ & $\begin{array}{c}\text { Glutathione, L- } \\
\text { Methionine, L-Cysteine }\end{array}$ & \\
\hline
\end{tabular}

Table C S4: A comparison of the proposed SERS based nano-biosensor with other SERS biosensors

\begin{tabular}{|c|c|c|c|c|c|}
\hline $\begin{array}{c}\text { Fabrication } \\
\text { methodology }\end{array}$ & Material synthesized & Self-activated & $\begin{array}{c}\text { Used for } \\
\text { Biomolecule }\end{array}$ & $\begin{array}{c}\text { Limit of } \\
\text { Detection }\end{array}$ & Reference \\
\hline Redox transmetalation & Prickly nickel nanowire & No & No & $10^{-5}$ & 129 \\
\hline Redox transmetalation & Ni nanoflowers & No & No & $10^{-5}$ & 138 \\
\hline Galvanic displacement & $\begin{array}{c}\text { Hybrid nickel } \\
\text { nanostructures }\end{array}$ & No & No & $10^{-11}$ & 137 \\
\hline Electrochemical & nanowire & No & No & $10^{-5}$ & 136 \\
\hline $\begin{array}{c}\text { Multiphoton } \\
\text { lonization } \\
\text { nanonetwork }\end{array}$ & $\begin{array}{c}\text { Yes } \\
\text { L-Cysteine, } \\
\text { L-Methionine, }\end{array}$ & $\begin{array}{c}\text { GSH - 10-12 } \\
\text { L-Cys - 10-6 } \\
\text { L-Met -10-6 }\end{array}$ & \\
\hline
\end{tabular}


Table C S5 - Laser parameters with corresponding synthesized nanonetwork image and Energy Dispersive X-ray image of observed nanonetwork

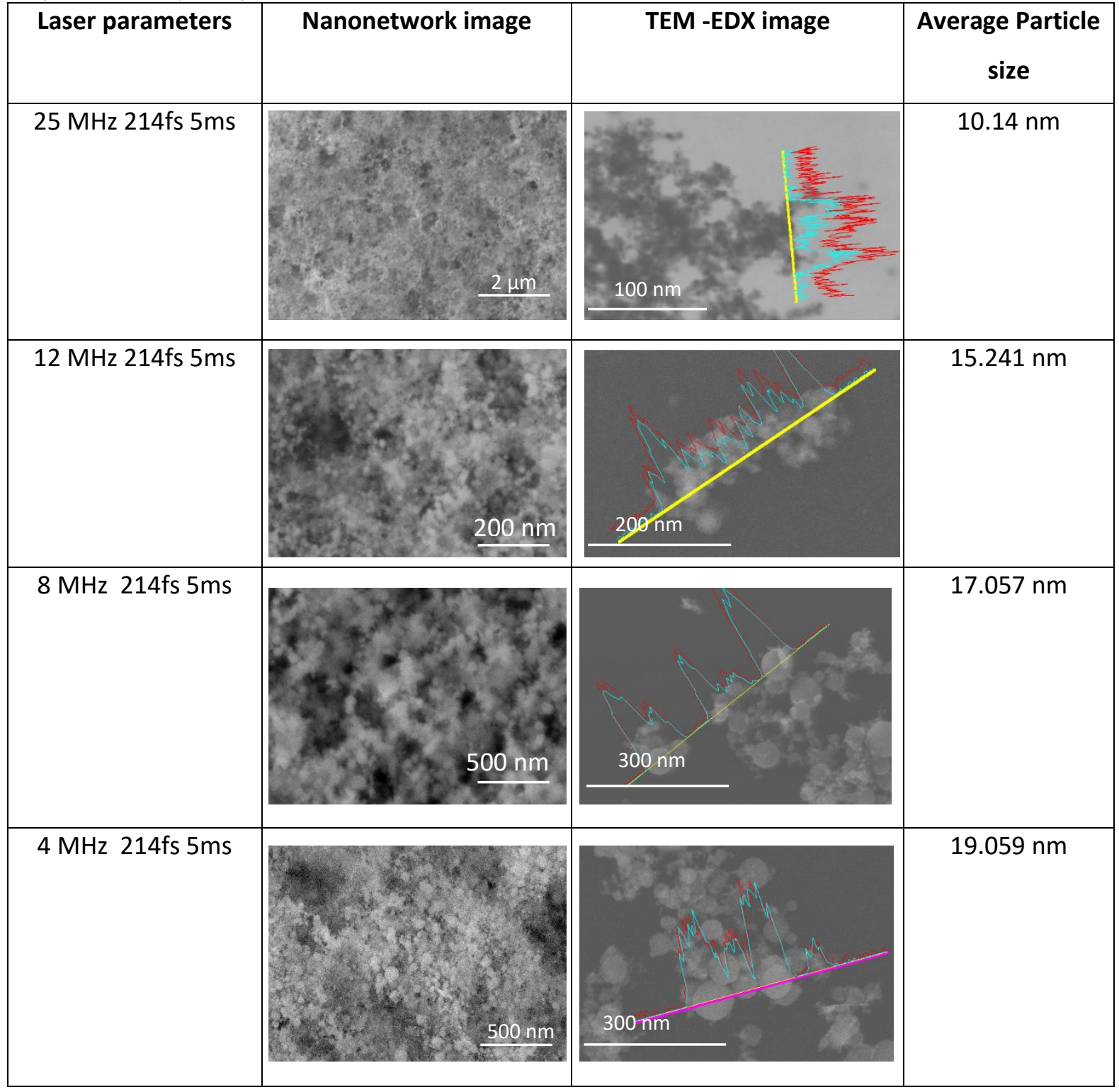




\section{REFERENCES}

1. Tupei, C. \& Liu, Y. Semiconductor Nanocrystals and Metal Nanoparticles. (2016).

2. Bhabra, G. et al. Nanoparticles can cause DNA damage across a cellular barrier. Nat. Nanotechnol. 4, 876-883 (2009).

3. Lu, A.-H., Salabas, E. L. \& Schüth, F. Magnetic Nanoparticles: Synthesis, Protection, Functionalization, and Application. Angew. Chemie Int. Ed. 46, 1222-1244 (2007).

4. Alagiri, M., Ponnusamy, S. \& Muthamizhchelvan, C. Synthesis and characterization of NiO nanoparticles by sol-gel method. J. Mater. Sci. Mater. Electron. 23, 728-732 (2011).

5. Silva, R. M. et al. Proteic sol-gel synthesis, structure and magnetic properties of $\mathrm{Ni} / \mathrm{NiO}$ core-shell powders. Ceram. Int. 44, 6152-6156 (2018).

6. Xu, C., Nie, D., Chen, H., Wang, Y. \& Liu, Y. Solvothermal synthesis of cauliflower-like CoNi microstructures with enhanced magnetic property. Mater. Lett. 142, 246-249 (2015).

7. Khansari, A., Enhessari, M. \& Salavati-Niasari, M. Synthesis and Characterization of Nickel Oxide Nanoparticles from Ni(salen) as Precursor. J. Clust. Sci. 24, 289-297 (2013).

8. Rostamnejadi, A. \& Bagheri, S. Optical, magnetic, and microwave properties of $\mathrm{Ni} / \mathrm{NiO}$ nanoparticles. Appl. Phys. A 123, 233 (2017).

9. Anandha Babu, G., Ravi, G., Navaneethan, M., Arivanandhan, M. \& Hayakawa, Y. An investigation of flower shaped $\mathrm{NiO}$ nanostructures by microwave and hydrothermal route. J. Mater. Sci. Mater. Electron. 25, 5231-5240 (2014).

10. Liu, B., Hu, Z., Che, Y., Chen, Y. \& Pan, X. Nanoparticle generation in ultrafast pulsed laser ablation of nickel. Appl. Phys. Lett. 90, 044103 (2007).

11. Nel, A. Toxic Potential of Materials. Science (80-. ). 311, 622-627 (2007).

12. Guo, H. et al. Research Advances on Pathways of Nickel-Induced Apoptosis. Int. J. Mol. Sci. 17, 118 (2015).

13. Duan, W.-X. et al. NiO nanoparticles induce apoptosis through repressing SIRT1 in human bronchial epithelial cells. Toxicol. Appl. Pharmacol. 286, 80-91 (2015).

14. Yoo, D., Lee, J.-H., Shin, T.-H. \& Cheon, J. Theranostic Magnetic Nanoparticles. Acc. Chem. Res. 44, 863-874 (2011).

15. Bhirde, A. A. et al. Targeted killing of cancer cells in vivo and in vitro with EGF-directed carbon nanotube-based drug delivery. ACS Nano 3, 307-316 (2009).

16. Reineck, P. \& Gibson, B. C. Near-Infrared Fluorescent Nanomaterials for Bioimaging and Sensing. Adv. Opt. Mater. 5, (2017). 
17. Singh, N. et al. Synthesis of Tunable and Multifunctional Ni-Doped Near-Infrared QDs for Cancer Cell Targeting and Cellular Sorting. Bioconjug. Chem. 23, 421-430 (2012).

18. Kang, H. et al. Near-Infrared SERS Nanoprobes with Plasmonic Au/Ag Hollow-Shell Assemblies for In Vivo Multiplex Detection. Adv. Funct. Mater. 23, 3719-3727 (2013).

19. Stobiecka, M. \& Hepel, M. Rapid functionalization of metal nanoparticles by moderator-tunable ligand-exchange process for biosensor designs. Sensors Actuators, B Chem. 149, 373-380 (2010).

20. Stobiecka, M. Novel plasmonic field-enhanced nanoassay for trace detection of proteins. Biosens. Bioelectron. 55, 379-385 (2014).

21. Wei, H. \& Xu, H. Hot spots in different metal nanostructures for plasmon-enhanced Raman spectroscopy. Nanoscale 5, 10794-805 (2013).

22. Lee, H., Shin, T. H., Cheon, J. \& Weissleder, R. Recent Developments in Magnetic Diagnostic Systems. Chem. Rev. 115, 10690-10724 (2015).

23. Maznichenko, D., Venkatakrishnan, K. \& Tan, B. Stimulating Multiple SERS Mechanisms by a Nano fi brous Three- Dimensional Network Structure of Titanium Dioxide (TiO 2 ). (2013).

24. Powell, J. A., Venkatakrishnan, K. \& Tan, B. Programmable SERS active substrates for chemical and biosensing applications using amorphous/crystalline hybrid silicon nanomaterial. Sci. Rep. 6, 19663 (2016).

25. Rai, M. et al. Enhanced Red Upconversion Emission, Magnetoluminescent Behavior, and Bioimaging Application of NaSc 0.75 Er 0.02 Yb 0.18 Gd 0.05 F 4 @AuNPs Nanoparticles. ACS Appl. Mater. Interfaces 7, 15339-15350 (2015).

26. Fratila, R. M., Rivera-Fernández, S. \& de la Fuente, J. M. Shape matters: synthesis and biomedical applications of high aspect ratio magnetic nanomaterials. Nanoscale 7, 8233-8260 (2015).

27. Tomitaka, A., Koshi, T., Hatsugai, S., Yamada, T. \& Takemura, Y. Magnetic characterization of surface-coated magnetic nanoparticles for biomedical application. J. Magn. Magn. Mater. 323, 1398-1403 (2011).

28. Liao, S. H. et al. Functionalized magnetic iron oxide/alginate core-shell nanoparticles for targeting hyperthermia. Int. J. Nanomedicine 10, 3315-3328 (2015).

29. Gobbo, O. L., Sjaastad, K., Radomski, M. W., Volkov, Y. \& Prina-Mello, A. Magnetic Nanoparticles in Cancer Theranostics. Theranostics 5, 1249-1263 (2015).

30. Wang, X. et al. Boosting the Peroxidase-Like Activity of Nanostructured Nickel by Inducing Its 3+ Oxidation State in LaNiO 3 Perovskite and Its Application for Biomedical Assays. Theranostics 7, 2277-2286 (2017). 
31. Mendoza-Garcia, A., Su, D. \& Sun, S. Sea urchin-like cobalt-iron phosphide as an active catalyst for oxygen evolution reaction. Nanoscale 8, 3244-3247 (2016).

32. Wu, L., Mendoza-Garcia, A., Li, Q. \& Sun, S. Organic Phase Syntheses of Magnetic Nanoparticles and Their Applications. Chem. Rev. 116, 10473-10512 (2016).

33. Nguyen, T. D. Portraits of colloidal hybrid nanostructures: Controlled synthesis and potential applications. Colloids Surfaces B Biointerfaces 103, 326-344 (2013).

34. Jun, Y., Seo, J. \& Cheon, J. Nanoscaling Laws of Magnetic Nanoparticles and Their Applicabilities in Biomedical Sciences. Acc. Chem. Res. 41, 179-189 (2008).

35. Barick, K. C. et al. Covalent Bridging of Surface Functionalized Fe3O4 and YPO4: Eu Nanostructures for Simultaneous Imaging and Therapy. Dalt. Trans. 44, 14686-14696 (2015).

36. Nazir, S., Hussain, T., Ayub, A., Rashid, U. \& MacRobert, A. J. Nanomaterials in combating cancer: Therapeutic applications and developments. Nanomedicine Nanotechnology, Biol. Med. 10, 1934 (2014).

37. Maher, S. et al. Multifunctional microspherical magnetic and $\mathrm{pH}$ responsive carriers for combination anticancer therapy engineered by droplet-based microfluidics. J. Mater. Chem. B 5, 4097-4109 (2017).

38. Wang, H. et al. Multifunctional PEG encapsulated Fe304@silver hybrid nanoparticles: antibacterial activity, cell imaging and combined photothermo/chemo-therapy. J. Mater. Chem. B 1,6225 (2013).

39. Chen, S. et al. Curcumin/sunitinib co-loaded BSA-stabilized SPIOs for synergistic combination therapy for breast cancer. J. Mater. Chem. B 5, 4060-4072 (2017).

40. Colombo, M. et al. Biological applications of magnetic nanoparticles. Chem. Soc. Rev. 41, 43064334 (2012).

41. Hayashi, K. et al. Magnetically Responsive Smart Nanoparticles for Cancer Treatment with a Combination of Magnetic Hyperthermia and Remote-Control Drug Release. Theranostics 4, 834844 (2014).

42. Wu, J., Zhou, W., Cheng, Q. \& Yang, J. Polyvinylpyrrolidone-stabilized magnetic nickel nanochains for cancer hyperthermia and catalysis applications. RSC Adv. 5, 22965-22971 (2015).

43. Alexiou, C. et al. Cancer therapy with drug loaded magnetic nanoparticles-magnetic drug targeting. J. Magn. Magn. Mater. 323, 1404-1407 (2011).

44. Mohammad, F. \& Azah, N. Journal of Colloid and Interface Science Doxorubicin-loaded magnetic gold nanoshells for a combination therapy of hyperthermia and drug delivery. J. Colloid Interface 
Sci. 434, 89-97 (2014).

45. An, N. et al. Gated magnetic mesoporous silica nanoparticles for intracellular enzyme-triggered drug delivery. Mater. Sci. Eng. C 69, 292-300 (2016).

46. An, X. et al. Rational Design of Multi-Stimuli-Responsive Nanoparticles for Precise Cancer Therapy. ACS Nano acsnano.6b01296 (2016). doi:10.1021/acsnano.6b01296

47. Felix Servin, L. P. Cytotoxic Effects of Nickel Nanowires in Human Fibroblasts. 3, 373-380 (2014).

48. Ahamed, M., Akhtar, M. J., Alhadlaq, H. A., Khan, M. A. M. \& Alrokayan, S. A. Comparative cytotoxic response of nickel ferrite nanoparticles in human liver HepG2 and breast MFC-7 cancer cells. Chemosphere 135, 278-88 (2015).

49. Cui, L. et al. Synthesis of Multifunctional Fe $304 @$ mSiO $2 @ A u$ Core-Shell Nanocomposites for pH-Responsive Drug Delivery. Eur. J. Inorg. Chem. 2014, 6156-6164 (2014).

50. Danhier, F., Feron, O. \& Préat, V. To exploit the tumor microenvironment: Passive and active tumor targeting of nanocarriers for anti-cancer drug delivery. J. Control. Release 148, 135-146 (2010).

51. Sudhasree, S., Shakila Banu, A., Brindha, P. \& Kurian, G. A. Synthesis of nickel nanoparticles by chemical and green route and their comparison in respect to biological effect and toxicity. Toxicol. Environ. Chem. 96, 743-754 (2014).

52. Ahamed, M., Ali, D., Alhadlaq, H. A. \& Akhtar, M. J. Nickel oxide nanoparticles exert cytotoxicity via oxidative stress and induce apoptotic response in human liver cells (HepG2). Chemosphere 93, 2514-2522 (2013).

53. Venning, F. A., Wullkopf, L. \& Erler, J. T. Targeting ECM Disrupts Cancer Progression. Front. Oncol. 5, 224 (2015).

54. Flaim, C. J., Chien, S. \& Bhatia, S. N. An extracellular matrix microarray for probing cellular differentiation. Nat. Methods 2, 119-125 (2005).

55. Gao, Y. et al. Three Dimensional and Homogenous Single Cell Cyclic Stretch within a Magnetic Micropillar Array (mMPA) for a Cell Proliferation Study. ACS Biomater. Sci. Eng. 2, 65-72 (2016).

56. Wang, Z. et al. Hierarchical Assembly of Bioactive Amphiphilic Molecule Pairs into Supramolecular Nanofibril Self-Supportive Scaffolds for Stem Cell Differentiation. J. Am. Chem. Soc. 138, 15027-15034 (2016).

57. Gamaly, E. G. \& Rode, a. V. Physics of ultra-short laser interaction with matter: From phonon excitation to ultimate transformations. Prog. Quantum Electron. 37, 215-323 (2013).

58. Tillack, M. S., Blair, D. W. \& Harilal, S. S. The effect of ionization on cluster formation in laser 
ablation plumes. Nanotechnology 15, 390-403 (2004).

59. Karmhag, R., Niklasson, G. A. \& Nygren, M. Oxidation kinetics of nickel nanoparticles. J. Appl. Phys. 89, 3012-3017 (2001).

60. Paeng, D. et al. Laser-Induced Reductive Sintering of Nickel Oxide Nanoparticles under Ambient Conditions. J. Phys. Chem. C 119, 6363-6372 (2015).

61. Maznichenko, D., Venkatakrishnan, K. \& Tan, B. Stimulating Multiple SERS Mechanisms by a Nanofibrous Three-Dimensional Network Structure of Titanium Dioxide (TiO 2 ). J. Phys. Chem. C 117, 578-583 (2013).

62. Gandhi, A. C. et al. Short-Range Magnon Excitation in NiO Nanoparticles. J. Phys. Chem. C 117, 18666-18674 (2013).

63. Stueker, O., Ortega, V. A., Goss, G. G. \& Stepanova, M. Understanding Interactions of Functionalized Nanoparticles with Proteins: A Case Study on Lactate Dehydrogenase. Small 10, 2006-2021 (2014).

64. Kim, S. et al. Characterization of the effects of silver nanoparticles on liver cell using HR-MAS NMR spectroscopy. Bull. Korean Chem. Soc. 32, 2021-2026 (2011).

65. Ingber, D. E. Tensegrity I. Cell structure and hierarchical systems biology. J. Cell Sci. 116, 11571173 (2003).

66. Albuschies, J. \& Vogel, V. The role of filopodia in the recognition of nanotopographies. Sci. Rep. 1658, 1-9 (2013).

67. Low, S. P., Williams, K. A., Canham, L. T. \& Voelcker, N. H. Evaluation of mammalian cell adhesion on surface-modified porous silicon. Biomaterials 27, 4538-4546 (2006).

68. Mattila, P. K. \& Lappalainen, P. Filopodia: molecular architecture and cellular functions. Nat. Rev. Mol. Cell Biol. 9, 446-454 (2008).

69. Lee, K., Lingampalli, N., Pisano, A. P., Murthy, N. \& So, H. Physical Delivery of Macromolecules using High-Aspect Ratio Nanostructured Materials. ACS Appl. Mater. Interfaces 7, 23387-23397 (2015).

70. Kenry, Chaudhuri, P. K., Loh, K. P. \& Lim, C. T. Selective Accelerated Proliferation of Malignant Breast Cancer Cells on Planar Graphene Oxide Films. ACS Nano 10, 3424-3434 (2016).

71. Zhang, L. et al. Controlled Propulsion and Cargo Transport of Rotating Nickel Nanowires near a Patterned Solid Surface. ACS Nano 4, 6228-6234 (2010).

72. Chen, T. et al. Smart multifunctional nanostructure for targeted cancer chemotherapy and magnetic resonance imaging. ACS Nano 5, 7866-7873 (2011). 
73. Momtazi, L. et al. Synthesis, characterization, and cellular uptake of magnetic nanocarriers for cancer drug delivery. J. Colloid Interface Sci. 433, 76-85 (2014).

74. Premnath, P., Tan, B. \& Venkatakrishnan, K. Ultrafast laser functionalized rare phased goldsilicon/silicon oxide nanostructured hybrid biomaterials. Colloids Surfaces B Biointerfaces 136, 828-837 (2015).

75. Tavangar, A., Tan, B. \& Venkatakrishnan, K. Synthesis of bio-functionalized three-dimensional titania nanofibrous structures using femtosecond laser ablation. Acta Biomater. 7, 2726-2732 (2011).

76. Chinnakkannu Vijayakumar, C., Venkatakrishnan, K. \& Tan, B. Harmonizing HeLa cell cytoskeleton behavior by multi-Ti oxide phased nanostructure synthesized through ultrashort pulsed laser. Sci. Rep. 5, 15294 (2015).

77. Kim, W., Ng, J. K., Kunitake, M. E., Conklin, B. R. \& Yang, P. Interfacing Silicon Nanowires with Mammalian Cells. J. Am. Chem. Soc. 129, 7228-7229 (2007).

78. Webster, M., Witkin, K. L. \& Cohen-Fix, O. Sizing up the nucleus: nuclear shape, size and nuclearenvelope assembly. J. Cell Sci. 122, 1477-1486 (2009).

79. Wang, Y. et al. Quantum-Dot-Based Theranostic Micelles Conjugated with an Anti-EGFR Nanobody for Triple-Negative Breast Cancer Therapy. ACS Appl. Mater. Interfaces 9, 3029730305 (2017).

80. Chinen, A. B. et al. Nanoparticle Probes for the Detection of Cancer Biomarkers, Cells, and Tissues by Fluorescence. Chem Rev 115, 10530-10574 (2015).

81. Wegner, K. D. \& Hildebrandt, N. Quantum dots: bright and versatile in vitro and in vivo fluorescence imaging biosensors. Chem. Soc. Rev. 44, 4792-4834 (2015).

82. Basov, D. N., Averitt, R. D. \& Hsieh, D. Towards properties on demand in quantum materials. Nat. Mater. 16, 1077-1088 (2017).

83. Pérez-Donoso, J. M. et al. Biomimetic, Mild Chemical Synthesis of CdTe-GSH Quantum Dots with Improved Biocompatibility. PLoS One 7, e30741 (2012).

84. Alivisatos, A. P. Semiconductor Clusters, Nanocrystals, and Quantum Dots. Science (80-. ). 271, 933-937 (1996).

85. Medintz, I. L., Uyeda, H. T., Goldman, E. R. \& Mattoussi, H. Quantum dot bioconjugates for imaging, labelling and sensing. Nat. Mater. 4, 435-446 (2005).

86. Huang, K. et al. Size-dependent localization and penetration of ultrasmall gold nanoparticles in cancer cells, multicellular spheroids, and tumors in vivo. ACS Nano 6, 4483-4493 (2012). 
87. Ruedas-Rama, M. J., Walters, J. D., Orte, A. \& Hall, E. A. H. Fluorescent nanoparticles for intracellular sensing: A review. Anal. Chim. Acta 751, 1-23 (2012).

88. Yu, X. et al. Dopant-controlled synthesis of water-soluble hexagonal NaYF4 nanorods with efficient upconversion fluorescence for multicolor bioimaging. Nano Res. 3, 51-60 (2010).

89. Das, K., Sarkar, S. \& Das, P. K. Fluorescent Indicator Displacement Assay: Ultrasensitive Detection of Glutathione and Selective Cancer Cell Imaging. ACS Appl. Mater. Interfaces 8, 25691-25701 (2016).

90. Chinnathambi, S., Chen, S., Ganesan, S. \& Hanagata, N. Silicon quantum dots for biological applications. Adv. Healthc. Mater. 3, 10-29 (2014).

91. Tang, F., Wang, C., Wang, X. \& Li, L. Facile Synthesis of Biocompatible Fluorescent Nanoparticles for Cellular Imaging and Targeted Detection of Cancer Cells. ACS Appl. Mater. Interfaces 7, 25077-25083 (2015).

92. Huang, G. et al. Facile integration of multiple magnetite nanoparticles for theranostics combining efficient MRI and thermal therapy. Nanoscale 7, 2667-2675 (2015).

93. Sun, Y. P. et al. Quantum-sized carbon dots for bright and colorful photoluminescence. J. Am. Chem. Soc. 128, 7756-7757 (2006).

94. Pramanik, A. et al. Fluorescent, Magnetic Multifunctional Carbon Dots for Selective Separation, Identification, and Eradication of Drug-Resistant Superbugs. ACS Omega 2, 554-562 (2017).

95. Bardhan, R., Lal, S., Joshi, A. \& Halas, N. J. Theranostic Nanoshells: From Probe Design to Imaging and Treatment of Cancer. Acc. Chem. Res. 44, 936-946 (2011).

96. Zou, F. et al. Dual-Mode SERS-Fluorescence Immunoassay Using Graphene Quantum Dot Labeling on One-Dimensional Aligned Magnetoplasmonic Nanoparticles. ACS Appl. Mater. Interfaces 7, 12168-12175 (2015).

97. De Los Santos Valladares, L. et al. Characterization of Ni thin films following thermal oxidation in air. J. Vac. Sci. Technol. B, Nanotechnol. Microelectron. Mater. Process. Meas. Phenom. 32, 051808 (2014).

98. Marciuš, M., Ristić, M., Ivanda, M. \& Musić, S. Formation and microstructure of nickel oxide films. J. Alloys Compd. 541, 238-243 (2012).

99. Chinnakkannu Vijayakumar, S., Venkatakrishnan, K. \& Tan, B. SERS Active Nanobiosensor Functionalized by Self-Assembled 3D Nickel Nanonetworks for Glutathione Detection. ACS Appl. Mater. Interfaces 9, 5077-5091 (2017).

100. Liu, S. et al. Controllable sulfuration engineered NiO nanosheets with enhanced capacitance for 
high rate supercapacitors. J. Mater. Chem. A 5, 4543-4549 (2017).

101. Zhen, W., Ma, J. \& Lu, G. Small-sized $\mathrm{Ni}\left(\begin{array}{lll}1 & 1 & 1\end{array}\right)$ particles in metal-organic frameworks with low over-potential for visible photocatalytic hydrogen generation. Appl. Catal. B Environ. 190, 12-25 (2016).

102. Sarkar, S. et al. Redox Transmetalation of Prickly Nickel Nanowires for Morphology Controlled Hierarchical Synthesis of Nickel/Gold Nanostructures for Enhanced Catalytic Activity and SERS Responsive Functional Material. J. Phys. Chem. C 115, 1659-1673 (2011).

103. Thema, F. T., Manikandan, E., Gurib-Fakim, A. \& Maaza, M. Single phase Bunsenite NiO nanoparticles green synthesis by Agathosma betulina natural extract. J. Alloys Compd. 657, 655661 (2016).

104. Das, A., Mandal, A. C., Roy, S. \& Nambissan, P. M. G. Positron annihilation studies of defects and fine size effects in nanocrystalline nickel oxide. J. Exp. Nanosci. 10, 622-639 (2015).

105. Patel, M., Kim, J. S., Kim, B. S., Kim, Y. H. \& Kim, J. Optical and photoelectrochemical properties of transparent NiO quantum dots. Mater. Lett. 218, 123-126 (2018).

106. Hong, W., Zhou, Y., Lv, C., Han, Z. \& Chen, G. NiO Quantum Dot Modified TiO 2 toward Robust Hydrogen Production Performance. ACS Sustain. Chem. Eng. acssuschemeng.7b03250 (2017). doi:10.1021/acssuschemeng.7b03250

107. El-Kemary, M., Nagy, N. \& El-Mehasseb, I. Nickel oxide nanoparticles: Synthesis and spectral studies of interactions with glucose. Mater. Sci. Semicond. Process. 16, 1747-1752 (2013).

108. Mironova-Ulmane, N. et al. Raman scattering in nanosized nickel oxide NiO. J. Phys. Conf. Ser. 93, 012039 (2007).

109. Gandhi, A. C., Cheng, H.-Y., Chang, Y.-M. \& Lin, J. G. Size confined magnetic phase in NiO nanoparticles. Mater. Res. Express 3, 035017 (2016).

110. Klein, N. D., Hurley, K. R., Feng, Z. V. \& Haynes, C. L. Dark field transmission electron microscopy as a tool for identifying inorganic nanoparticles in biological matrices. Anal. Chem. 87, 4356-4362 (2015).

111. Yallapu, M. M. et al. Curcumin-loaded magnetic nanoparticles for breast cancer therapeutics and imaging applications. Int. J. Nanomedicine 7, 1761-1779 (2012).

112. Albanese, A. et al. Secreted biomolecules alter the biological identity and cellular interactions of nanoparticles. ACS Nano 8, 5515-5526 (2014).

113. Chithrani, B. D., Ghazani, A. A. \& Chan, W. C. W. Determining the size and shape dependence of gold nanoparticle uptake into mammalian cells. Nano Lett. 6, 662-668 (2006). 
114. Behzadi, S. et al. Cellular uptake of nanoparticles: journey inside the cell. Chem. Soc. Rev. 46, 4218-4244 (2017).

115. Gu, F. X. et al. Targeted nanoparticles Over the past decade, there has been an increasing interest in using fabrication of targeted NPs using microfluidic devices . Nano Today 2, 14-21 (2007).

116. Oh, N. \& Park, J. H. Endocytosis and exocytosis of nanoparticles in mammalian cells. Int. J. Nanomedicine 9, 51-63 (2014).

117. Elkin, S. R. et al. A systematic analysis reveals heterogeneous changes in the endocytic activities of cancer cells. Cancer Res. 75, 4640-4650 (2015).

118. McMahon, H. T. \& Boucrot, E. Molecular mechanism and physiological functions of clathrinmediated endocytosis. Nat. Rev. Mol. Cell Biol. 12, 517-533 (2011).

119. Chithrani, B. D. \& Chan, W. C. W. Elucidating the mechanism of cellular uptake and removal of protein-coated gold nanoparticles of different sizes and shapes. Nano Lett. 7, 1542-1550 (2007).

120. Yallapu, M. M. et al. Multi-functional magnetic nanoparticles for magnetic resonance imaging and cancer therapy. Biomaterials 32, 1890-1905 (2011).

121. Sugawa, K. et al. Metal-enhanced fluorescence platforms based on plasmonic ordered copper arrays: Wavelength dependence of quenching and enhancement effects. ACS Nano 7, 999710010 (2013).

122. Chen, J. et al. Specific detection of cancer cells through aggregation-induced emission of a lightup bioprobe. Chem. Commun. 53, 2398-2401 (2017).

123. Khandelwal, P. \& Poddar, P. Fluorescent metal quantum clusters: an updated overview of the synthesis, properties, and biological applications. J. Mater. Chem. B 5, 9055-9084 (2017).

124. Liu, B. \& Liu, J. Comprehensive Screen of Metal Oxide Nanoparticles for DNA Adsorption, Fluorescence Quenching, and Anion Discrimination. ACS Appl. Mater. Interfaces 7, 24833-24838 (2015).

125. Lv, Y. et al. Nanoplatform Assembled from a CD44-Targeted Prodrug and Smart Liposomes for Dual Targeting of Tumor Microenvironment and Cancer Cells. ACS Nano (2018). doi:10.1021/acsnano.7b08051

126. Hossain, M. Z. \& Kleve. Nickel nanowires induced and reactive oxygen species mediated apoptosis in human pancreatic adenocarcinoma cells. Int. J. Nanomedicine 6, 1475 (2011).

127. Wang, Y. et al. Nickel-Refining Fumes Induced DNA Damage and Apoptosis of NIH/3T3 Cells via Oxidative Stress. Int. J. Environ. Res. Public Health 13, 629 (2016). 
128. Pan, J. et al. Reactive oxygen species-activated Akt/ASK1/p38 signaling pathway in nickel compound-induced apoptosis in BEAS 2B cells. Chem. Res. Toxicol. 23, 568-577 (2010).

129. Manikandan, M., Nasser Abdelhamid, H., Talib, A. \& Wu, H. F. Facile synthesis of gold nanohexagons on graphene templates in Raman spectroscopy for biosensing cancer and cancer stem cells. Biosens. Bioelectron. 55, 180-186 (2014).

130. Saxena, U. \& Das, A. B. Nanomaterials towards fabrication of cholesterol biosensors: Key roles and design approaches. Biosens. Bioelectron. 75, 196-205 (2016).

131. Kang, Z. et al. Enhanced photoelectrochemical property of $\mathrm{ZnO}$ nanorods array synthesized on reduced graphene oxide for self-powered biosensing application. Biosens. Bioelectron. 64, 499504 (2014).

132. Alubaidy, M., Soleymani, L., Venkatakrishnan, K. \& Tan, B. Femtosecond laser nanostructuring for femtosensitive DNA detection. Biosens. Bioelectron. 33, 82-87 (2012).

133. Stobiecka, M. \& Hepel, M. Effect of buried potential barrier in label-less electrochemical immunodetection of glutathione and glutathione-capped gold nanoparticles. Biosens. Bioelectron. 26, 3524-3530 (2011).

134. Ding, Y., Liu, J., Wang, H., Shen, G. \& Yu, R. A piezoelectric immunosensor for the detection of $\alpha-$ fetoprotein using an interface of gold/hydroxyapatite hybrid nanomaterial. Biomaterials 28, 2147-2154 (2007).

135. Buryakov, I. A., Buryakov, T. I. \& Matsayev, V. T. Electrical, electrochemical, and thermometric sensors for the detection of explosives. J. Anal. Chem. 71, 234-242 (2016).

136. Culha, M., Cullum, B., Lavrik, N. \& Klutse, C. K. Surface-Enhanced Raman Scattering as an Emerging Characterization and Detection Technique. J. Nanotechnol. 2012, 1-15 (2012).

137. Li, W. et al. CuTe Nanocrystals: Shape and Size Control, Plasmonic Properties, and Use as SERS Probes and Photothermal Agents. J. Am. Chem. Soc. 135, 7098-7101 (2013).

138. Register, J. K. et al. In vivo detection of SERS-encoded plasmonic nanostars in human skin grafts and live animal models. Anal. Bioanal. Chem. 407, 8215-8224 (2015).

139. Xu, W. et al. Surface enhanced Raman spectroscopy on a flat graphene surface. Proc. Natl. Acad. Sci. 109, 9281-9286 (2012).

140. Zhang, Y. et al. Nanocap array of Au:Ag composite for surface-enhanced Raman scattering. Spectrochim. Acta - Part A Mol. Biomol. Spectrosc. 152, 461-467 (2016).

141. Mao, Z. et al. Metal-Semiconductor Contacts Induce the Charge-Transfer Mechanism of SurfaceEnhanced Raman Scattering. J. Phys. Chem. C 115, 18378-18383 (2011). 
142. Lombardi, J. R. \& Birke, R. L. The theory of surface-enhanced Raman scattering. J. Chem. Phys. 136, 144704 (2012).

143. Wang, D., Zhu, W., Best, M. D., Camden, J. P. \& Crozier, K. B. Directional Raman Scattering from Single Molecules in the Feed Gaps of Optical Antennas. Nano Lett. 13, 2194-2198 (2013).

144. Cortés, E. et al. Monitoring the Electrochemistry of Single Molecules by Surface-Enhanced Raman Spectroscopy. J. Am. Chem. Soc. 132, 18034-18037 (2010).

145. Sarkar, S., Dutta, S. \& Pal, T. Tailored "Sandwich" Strategy in Surface Enhanced Raman Scattering: Case Study with para -Phenylenediamine and Application in Femtomolar Detection of Melamine. J. Phys. Chem. C 118, 28152-28161 (2014).

146. Wen, C. et al. Bi-functional ZnO-RGO-Au substrate: photocatalysts for degrading pollutants and SERS substrates for real-time monitoring. Chem. Commun. 49, 3049 (2013).

147. Zhong, Z. et al. Controlled organization of au colloids into linear assemblies. J. Phys. Chem. B 108, 18119-18123 (2004).

148. Li, Y., Liu, Q. \& Shen, W. Morphology-dependent nanocatalysis: metal particles. Dalt. Trans. 40, 5811 (2011).

149. Shen, H. et al. Shape effect on a single-nanoparticle-based plasmonic nanosensor. Nanotechnology 24, 285502 (2013).

150. Wang, X., Shi, W., She, G. \& Mu, L. Surface-Enhanced Raman Scattering (SERS) on transition metal and semiconductor nanostructures. Phys. Chem. Chem. Phys. 14, 5891 (2012).

151. Kumar, G. V.P. Plasmonic nano-architectures for surface enhanced Raman scattering: a review. J. Nanophotonics 6, 064503 (2012).

152. Bayata, F., Akinci, Z. B., Donatan, A. S. \& Urgen, M. A novel free-standing nanowire substrate with surface enhanced Raman scattering (SERS) activity. Mater. Lett. 67, 387-389 (2012).

153. Sajanlal, P. R. \& Pradeep, T. Functional hybrid nickel nanostructures as recyclable SERS substrates: detection of explosives and biowarfare agents. Nanoscale 4, 3427 (2012).

154. Manoj, K., Gayathri, S., Jayabal, P. \& Ramakrishnan, V. Synthesis and characterization of Ni/Ag nanocomposite for surface enhanced Raman scattering measurement. Mater. Res. Express 2, 065003 (2015).

155. Sivakumar, M., Venkatakrishnan, K. \& Tan, B. Study of metallic fibrous nanoparticle aggregate produced using femtosecond laser radiation under ambient conditions. Nanotechnology 21, 225601 (2010).

156. Sivakumar, M., Venkatakrishnan, K. \& Tan, B. Characterization of MHz pulse repetition rate 
femtosecond laser-irradiated gold-coated silicon surfaces. Nanoscale Res. Lett. 6, 78 (2011).

157. Shoute, L. C. T., Bergren, A. J., Mahmoud, A. M., Harris, K. D. \& McCreery, R. L. Optical interference effects in the design of substrates for surface-enhanced raman spectroscopy. Appl. Spectrosc. 63, 133-140 (2009).

158. Jensen, T. et al. Electrodynamics of noble metal nanoparticles and nanoparticle clusters. J. Clust. Sci. 10, 295-317 (1999).

159. Gandhi, A. et al. Growth mechanism and magnon excitation in NiO nanowalls. Nanoscale Res. Lett. 6, 485 (2011).

160. Yamada, H. \& Yamamoto, Y. Surface enhanced Raman scattering (SERS) of chemisorbed species on various kinds of metals and semiconductors. Surf. Sci. 134, 71-90 (1983).

161. Balendiran, G. K., Dabur, R. \& Fraser, D. The role of glutathione in cancer. Cell Biochem. Funct. 22, 343-352 (2004).

162. Meister, A. \& Anderson, M. E. Glutathione. Annu. Rev. Biochem. 52, 711-760 (1983).

163. Bains, J. S. \& Shaw, C. A. Neurodegenerative disorders in humans: The role of glutathione in oxidative stress-mediated neuronal death. Brain Res. Rev. 25, 335-358 (1997).

164. Han, B., Yuan, J. \& Wang, E. Sensitive and selective sensor for biothiols in the cell based on the recovered fluorescence of the CdTe quantum dots-Hg(II) system. Anal. Chem. 81, 5569-5573 (2009).

165. Zhang, X., Wu, F. G., Liu, P., Gu, N. \& Chen, Z. Enhanced fluorescence of gold nanoclusters composed of $\mathrm{HAuCl} 4$ and histidine by glutathione: Glutathione detection and selective cancer cell imaging. Small 10, 5170-5177 (2014).

166. Tian, D., Qian, Z., Xia, Y. \& Zhu, C. Gold Nanocluster-Based Fluorescent Probes for Near-Infrared and Turn-On Sensing of Glutathione in Living Cells. Langmuir 28, 3945-3951 (2012).

167. Huang, G. G., Han, X. X., Hossain, M. K. \& Ozaki, Y. Development of a Heat-Induced SurfaceEnhanced Raman Scattering Sensing Method for Rapid Detection of Glutathione in Aqueous Solutions. Anal. Chem. 81, 5881-5888 (2009).

168. Larsson, M. \& Lindgren, J. Analysis for glutathione and immunoglobulin $G$ inside chromatographic beads using surface-enhanced Raman scattering spectroscopy. J. Raman Spectrosc. 36, 394-399 (2005).

169. Saha, A. \& Jana, N. R. Detection of cellular glutathione and oxidized glutathione using magneticplasmonic nanocomposite-based 'turn-Off' surface enhanced raman scattering. Anal. Chem. 85, 9221-9228 (2013). 
170. Cai, Q.-Y. et al. A rapid fluorescence 'switch-on' assay for glutathione detection by using carbon dots-MnO2 nanocomposites. Biosens. Bioelectron. 72, 31-6 (2015).

171. Martinez-Garcia, M. M., Cardoso-Avila, P. E. \& Pichardo-Molina, J. L. Concave gold nanocubes on Al-6063 alloy as a simple and efficient SERS substrate. Colloids Surfaces A Physicochem. Eng. Asp. 493, 66-73 (2016).

172. Dou, X., Jung, Y. M., Cao, Z.-Q. \& Ozaki, Y. Surface-Enhanced Raman Scattering of Biological Molecules on Metal Colloid II: Effects of Aggregation of Gold Colloid and Comparison of Effects of pH of Glycine Solutions between Gold and Silver Colloids. Appl. Spectrosc. 53, 1440-1447 (1999).

173. Jing, C. \& Fang, Y. Simple method for electrochemical preparation of silver dendrites used as active and stable SERS substrate. J. Colloid Interface Sci. 314, 46-51 (2007).

174. Guo, Z. et al. A molecular-gap device for specific determination of mercury ions. Sci. Rep. 3, 3034 (2013).

175. Brambilla, a. et al. Adapting and testing a portable Raman spectrometer for SERS analysis of amino acids and small peptides. J. Mol. Struct. 1044, 121-127 (2013).

176. Der, A. et al. Study of Optical Properties of a Glutathione Capped Gold Nanoparticles using Linker ( MHDA ) by Fourier Transform Infra Red Spectroscopy and Surface Enhanced Raman Scattering. Int. J. Biol. Biomol. Agric. Food Biotechnol. Eng. 7, 1-4 (2013).

177. Lv, M., Gu, H., Yuan, X., Gao, J. \& Cai, T. Investigation of 3D silvernanodendrite@glass as surfaceenhanced Raman scattering substrate for the detection of Sildenafil and GSH. J. Mol. Struct. 1029, 75-80 (2012).

178. Qian, K., Liu, H., Yang, L. \& Liu, J. Designing and fabricating of surface-enhanced Raman scattering substrate with high density hot spots by polyaniline template-assisted self-assembly. Nanoscale 4, 6449 (2012).

179. Lee, H. K. et al. Superhydrophobic surface-enhanced Raman scattering platform fabricated by assembly of Ag nanocubes for trace molecular sensing. ACS Appl. Mater. Interfaces 5, 1140911418 (2013).

180. He, D., Hu, B., Yao, Q., Wang, K. \& Yu, S. Large-Scale Synthesis of Flexible Free- Sensitivity : Electrospun PVA Nanofibers of Silver Nanoparticles. ACS Nano 3, 3993-4002 (2009).

181. Li, J., Chen, L., Lou, T. \& Wang, Y. Highly Sensitive SERS Detection of As3+ Ions in Aqueous Media using Glutathione Functionalized Silver Nanoparticles. ACS Appl. Mater. Interfaces 3, 3936-3941 (2011).

182. Liu, R. et al. A simple method for preparation of Ag nanofilm used as active, stable, and 
biocompatible SERS substrate by using electrostatic self-assembly. J. Colloid Interface Sci. 343, 52-57 (2010).

183. Lee, J. et al. Particle-Film Plasmons on Periodic Silver Film over Nanosphere (AgFON): A Hybrid Plasmonic Nanoarchitecture for Surface-Enhanced Raman Spectroscopy. ACS Appl. Mater. Interfaces 8, 634-642 (2016).

184. Guan, Z. et al. Band-Selective Coupling-Induced Enhancement of Two-Photon Photoluminescence in Gold Nanocubes and Its Application as Turn-on Fluorescent Probes for Cysteine and Glutathione. ACS Appl. Mater. Interfaces 4, 5711-5716 (2012).

185. Podstawka, E., Ozaki, Y. \& Proniewicz, L. M. Part I: Surface-enhanced Raman spectroscopy investigation of amino acids and their homodipeptides adsorbed on colloidal silver. Appl. Spectrosc. 58, 570-580 (2004).

186. Pawlukojć, A., Leciejewicz, J., Ramirez-Cuesta, A. J. \& Nowicka-Scheibe, J. I-Cysteine: Neutron spectroscopy, Raman, IR and ab initio study. Spectrochim. Acta Part A Mol. Biomol. Spectrosc. 61, 2474-2481 (2005).

187. Edsall, J. T., Otvos, J. W. \& Rich, A. Raman Spectra of Amino Acids and Related Compounds. VII. Glycylglycine, Cysteine, Cystine and Other Amino Acids. J. Am. Chem. Soc. 72, 474-477 (1950).

188. Zhu, G., Zhu, X., Fan, Q. \& Wan, X. Raman spectra of amino acids and their aqueous solutions. Spectrochim. Acta - Part A Mol. Biomol. Spectrosc. 78, 1187-1195 (2011).

189. Pandiarajan, S., Umadevi, M., Briget Mary, M., Rajaram, R. K. \& Ramakrishnan, V. Infrared and Raman spectroscopic studies ofL-methioninium nitrate. J. Raman Spectrosc. 35, 907-913 (2004).

190. Ghazaryan, V. V, Zakharov, B. A., Boldyreva, E. V \& Petrosyan, A. M. L-Methioninium picrate. Spectrochim. Acta - Part A Mol. Biomol. Spectrosc. 142, 344-349 (2015).

191. Zhong, J. H. et al. Probing the electronic and catalytic properties of a bimetallic surface with $3 \mathrm{~nm}$ resolution. Nat. Nanotechnol. 12, 132-136 (2017).

192. Richard-Lacroix, M., Zhang, Y., Dong, Z. \& Deckert, V. Mastering high resolution tip-enhanced Raman spectroscopy: towards a shift of perception. Chem. Soc. Rev. 46, 3922-3944 (2017).

193. Huang, B., Bates, M. \& Zhuang, X. Super resolution fluorescence microscopy. Annu. Rev. Biochem. 78, 993-1016 (2010).

194. Jiang, S. et al. Subnanometer-resolved chemical imaging via multivariate analysis of tip-enhanced Raman maps. Light Sci. Appl. 6, e17098 (2017).

195. Zrimsek, A. B. et al. Single-Molecule Chemistry with Surface- and Tip-Enhanced Raman Spectroscopy. Chem. Rev. 117, 7583-7613 (2017). 
196. Chen, C., Hayazawa, N. \& Kawata, S. A $1.7 \mathrm{~nm}$ resolution chemical analysis of carbon nanotubes by tip-enhanced Raman imaging in the ambient. Nat. Commun. 5, 1-5 (2014).

197. Xiao, L. \& Schultz, Z. D. Targeted-TERS detection of integrin receptors on human cancer cells. Cancer Cell Microenviron. 3-8 (2016). doi:10.14800/ccm.1419

198. Bonhommeau, S., Talaga, D., Hunel, J., Cullin, C. \& Lecomte, S. Tip-Enhanced Raman Spectroscopy to Distinguish Toxic Oligomers from $A_{1-42}$ Fibrils at the Nanometer Scale. Angew. Chemie Int. Ed. 56, 1771-1774 (2017).

199. Singhal, R. et al. Multifunctional carbon-nanotube cellular endoscopes. Nat. Nanotechnol. 6, 5764 (2011).

200. Vitol, E. A. et al. In Situ Intracellular Spectroscopy with Surface Enhanced Raman Spectroscopy (SERS)-Enabled Nanopipettes. ACS Nano 3, 3529-3536 (2009).

201. Yamazoe, S. et al. Large-area surface-enhanced raman spectroscopy imaging of brain ischemia by gold nanoparticles grown on random nanoarrays of transparent boehmite. ACS Nano 8, 56225632 (2014).

202. Yan, Z. et al. A facile high-performance SERS substrate based on broadband near-perfect optical absorption. J. Raman Spectrosc. 46, 795-801 (2015).

203. Ding, Q., Ma, Y., Ye, Y., Yang, L. \& Liu, J. A simple method to prepare the magnetic Ni@Au coreshell nanostructure for the cycle surface enhanced Raman scattering substrates. J. Raman Spectrosc. 44, 987-993 (2013).

204. Tao, X. J. et al. A new approach to synthesize nickel nanoparticles. Acta Physico-Chimica Sin. 21, 569-572 (2005).

205. Powell, J. A., Venkatakrishnan, K. \& Tan, B. Towards universal SERS detection of disease signalling bioanalytes using 3D self-assembled non-plasmonic near-quantum scale silicon probe. ACS Appl. Mater. Interfaces acsami.7b15393 (2017). doi:10.1021/acsami.7b15393

206. Chinnakkannu Vijayakumar, S., Venkatakrishnan, K. \& Tan, B. Bio-functionalizing heterogeneous phase activated titanium by multiphoton ionization energy mechanism to harmonize cell proliferative behavior. Mater. Sci. Eng. C 76, (2017).

207. Kleinman, S. L. et al. Single-molecule surface-enhanced raman spectroscopy of crystal violet isotopologues: Theory and experiment. J. Am. Chem. Soc. 133, 4115-4122 (2011).

208. Deckert, V., Deckert-Gaudig, T., Richter, M., Treffer, R. \& Lin, X. Label free investigation of biomolecules on the nanometer scale using tip-enhanced Raman spectroscopy. Prog. Biomed. Opt. Imaging - Proc. SPIE 7560, (2010). 
209. Hajba, L. \& Guttman, A. The use of magnetic nanoparticles in cancer theranostics: Toward handheld diagnostic devices. Biotechnol. Adv. 34, 1-8 (2015).

210. Treuel, L., Jiang, X. \& Nienhaus, G. U. New views on cellular uptake and trafficking of manufactured nanoparticles. J. R. Soc. Interface 10, 20120939 (2013).

211. Veiseh, O., Gunn, J. W. \& Zhang, M. Design and fabrication of magnetic nanoparticles for targeted drug delivery and imaging. Adv. Drug Deliv. Rev. 62, 284-304 (2010).

212. Raemy, D. O. et al. Cerium oxide nanoparticle uptake kinetics from the gas-phase into lung cells in vitro is transport limited. Eur. J. Pharm. Biopharm. 77, 368-375 (2011).

213. Wei, L. et al. Live-cell imaging of alkyne-tagged small biomolecules by stimulated Raman scattering. Nat. Methods 11, 410-412 (2014).

214. Hamada, K. et al. Raman microscopy for dynamic molecular imaging of living cells. J. Biomed. Opt. 13, 044027 (2008). 